\title{
New broadband, low cost and compact MIMO radar frontends.
}
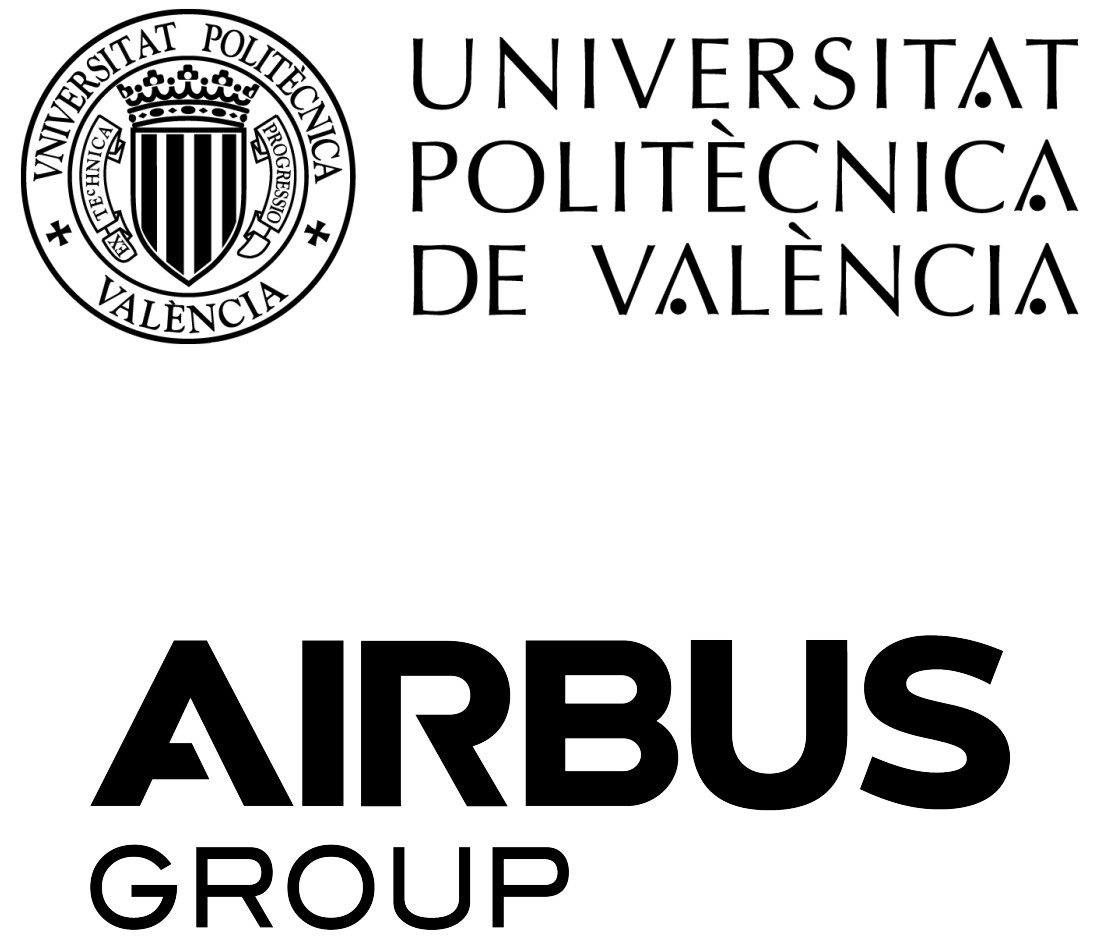

Valencia May 2018

First director: Héctor Esteban González

Second director: Ángel Belenguer Martínez

Industrial director: Volker Ziegler 



\title{
New broadband, low cost and compact MIMO radar frontends.
}

\author{
Enric Miralles Navarro
}

\author{
A Dissertation \\ Presented to the Academic Faculty of \\ Universitat Politècnica de València \\ In Partial Fulfillment \\ of the Requirements for the Degree \\ Doctor of Philosophy
}

Valencia, 15-05-2018 
First director: Héctor Esteban González

Second director: Ángel Belenguer Martínez Industrial director: Volker Ziegler 


\section{Contents}

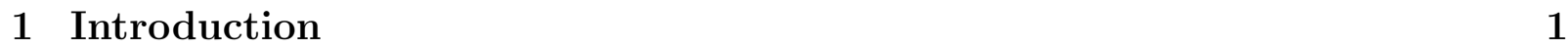

1.1 Motivation . . . . . . . . . . . . . . . . . . . . . . 1

1.2 State of the Art . . . . . . . . . . . . . . . . . . . . 2

1.3 Objectives . . . . . . . . . . . . . . . . . . . . . . . . . . . . . . . . . . . . . . . . .

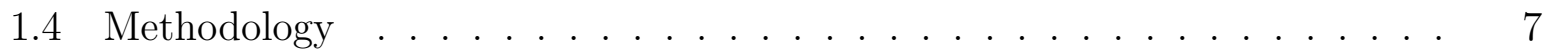

1.5 Structure of the Work . . . . . . . . . . . . . . . . . . . . . . 8

2 General Review of MIMO Radar $\quad 15$

2.1 Overview . . . . . . . . . . . . . . . . . . . . . 15

2.2 Frequency Modulated Continuous Wave Radar . . . . . . . . . . . . . . . . 15

2.3 Angle Estimation Basics . . . . . . . . . . . . . . . . . . . . . . . 18

2.4 Principle of the MIMO Radar . . . . . . . . . . . . . . . . . . . . . . . . . . . . . . . . . . . . . . . . . . 20

2.5 Time Division Multiplexing . . . . . . . . . . . . . . . . . . . . . . . 21

3 A MIMO Radar Implementation $\quad 25$

3.1 System Architecture . . . . . . . . . . . . . . . . . . . . . 25

3.2 MIMO Array Configuration . . . . . . . . . . . . . . . . . . . . . . 27

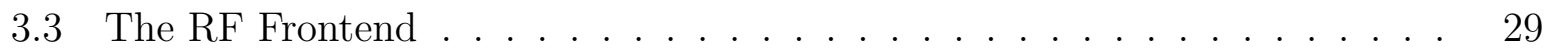

3.4 Building Blocks Design . . . . . . . . . . . . . . . . . . . . . . . . . . . . . . . . . . . . . . 30

3.4 .1 Calibration Kit Design . . . . . . . . . . . . . . . . . . . . . . . . 30

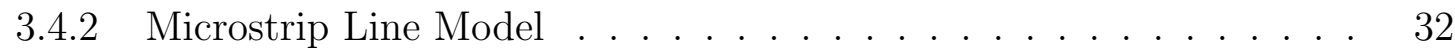

3.4 .3 Amplifiers . . . . . . . . . . . . . . . . . . . . 34

3.4 .4 Power Divider . . . . . . . . . . . . . . . . . . . . . . . . . . . . . . 36

3.4 .5 RF Switch . . . . . . . . . . . . . . . . . . . . . . . . 38

3.5 LO Distribution Board . . . . . . . . . . . . . . . . . . . . . . . . . . 39

3.5 .1 LO Distribution Board: System Level Considerations . . . . . . . . 40

3.5.2 LO Distribution Board: Simulation and Measurement . . . . . . . . 40

3.6 Transmitter Board . . . . . . . . . . . . . . . . . . . . . 45

3.6.1 Transmitter Board: System Level Considerations . . . . . . . . . . 45

3.6.2 Transmitter Board: Simulation and Measurement . . . . . . . . . . 47

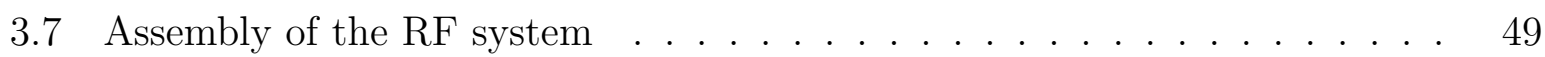

3.8 System Performance and Image Results . . . . . . . . . . . . . . . . . . . . 51

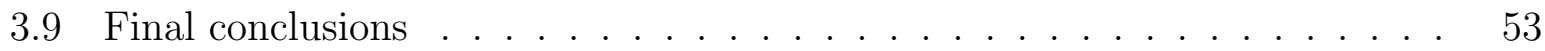


\begin{tabular}{|lll}
\hline A Compact Multifuctional MIMO Radar & 57
\end{tabular}

4.1 System Architecture . . . . . . . . . . . . . . . . . . . . . . 58

4.2 MIMO Array Configuration . . . . . . . . . . . . . . . . . . . . . . . . . 60

4.3 The RF Frontend . . . . . . . . . . . . . . . . . . . . . . . . . . . . 63

4.4 RF Components Design . . . . . . . . . . . . . . . . . . . . . 64

4.4 .1 Resonant Slot Patch Antennas . . . . . . . . . . . . . . . . . . . . . 64

4.4 .2 Vertical Transition . . . . . . . . . . . . . . . . . . . . . 77

$4.4 .3 \quad$ Three-Way Planar Wilkinson Power Divider . . . . . . . . . . . . . 79

4.5 Antenna board . . . . . . . . . . . . . . . . . . . . . 82

4.5 .1 Antenna board: Measurements . . . . . . . . . . . . . . . 86

4.6 System Performance and Image Results . . . . . . . . . . . . . . . . . . . . . . . 87

4.6 .1 Angular resolution . . . . . . . . . . . . . . . . . . . . 88

4.6 .2 Radar field of view . . . . . . . . . . . . . . . . . . . . . . . . . 89

4.6 .3 Zone surveillance applications . . . . . . . . . . . . . . . . . . . 91

4.7 Final conclusions $\ldots \ldots \ldots 5$

\begin{tabular}{|lll}
5 & Very wideband components for radar applications. & 99
\end{tabular}

5.1 Basics of Traditional Wilkinson Dividers . . . . . . . . . . . . . . . . . . 100

5.2 Broadband Wilkinson . . . . . . . . . . . . . . . . . . . . . . . . . . 100

5.2 .1 Improvements of Tapered Wilkinson Divider . . . . . . . . . . . . . 101

$5.2 .2 \quad$ Circuit model . . . . . . . . . . . . . . . . . . . . 102

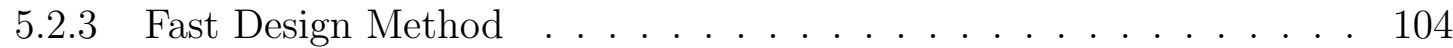

5.2 .4 Measured Results . . . . . . . . . . . . . . . . . . . . . . . . 106

5.3 A 3D Printed PCB integrated TEM Horn

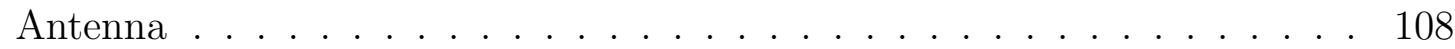

5.3 .1 Basics of TEM Horn Antennas . . . . . . . . . . . . . . . . . . . . . 109

5.3 .2 Antena concept . . . . . . . . . . . . . . . . . . 109

$5.3 .3 \quad$ MSL to PPW transition . . . . . . . . . . . . . . . . . . . . . . 110

5.3 .4 Antenna Results . . . . . . . . . . . . . . . . . . . 116

5.3 .5 Final Conclusions . . . . . . . . . . . . . . . . 118

$6 \quad$ ESIW for MIMO Radar applications 123

6.1 ESIW Slotted Antenna . . . . . . . . . . . . . . . . . . . . . . . 123

6.1 .1 Waveguide Selection . . . . . . . . . . . . . . . . . 123

6.1 .2 Layer Stack-up selection . . . . . . . . . . . . . . . . . . . . . . . . 124

6.1 .3 3D full-wave Simulation . . . . . . . . . . . . . . . . . . . . . . 125

6.1 .4 Measured Results . . . . . . . . . . . . . . . . . . 125

6.2 Cross-guide Moreno directional coupler in empty substrate integrated waveg-

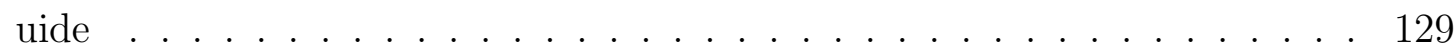

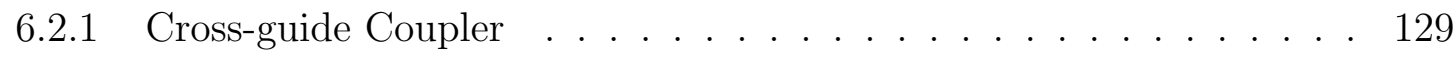

6.2 .2 Implementation in ESIW . . . . . . . . . . . . . . . . . . . . . . . . 130

$6.2 .3 \quad$ Specifications And Design . . . . . . . . . . . . . . . . . . . 132

6.2 .4 Results . . . . . . . . . . . . . . . . . . . . . . 132 
6.2 .5 Final Conslusions . . . . . . . . . . . . . . . . . . . . . . . . 135

\begin{tabular}{|lll}
\hline & Summary and Future Lines of Research & 139
\end{tabular}

7.1 Summary . . . . . . . . . . . . . . . . . . . . . 139

7.2 Resumen . . . . . . . . . . . . . . . . . . . . . . . . . 140

7.3 Resum . . . . . . . . . . . . . . . . . . . . . . 141

7.4 Future Lines of Research . . . . . . . . . . . . . . . . . . . . . . . . . . . . 142

7.5 List of Contributions . . . . . . . . . . . . . . . . . . . . . . . . . . . . . . 142

7.6 List of abbreviations . . . . . . . . . . . . . . . . . . . . . . . 144

$\begin{array}{lr}\text { Gracias. } & 149\end{array}$ 


\section{List of Figures}

1.1 Schematic representation of the MIMO antenna configuration with the physical array (left) showing the 16 TX antennas in blue, the 16 RX antennas in red and the resulting virtual array (right). . . . . . . . . . . . . . . 3

1.2 Block diagram of a 3D Imaging FMCW 24x24 MIMO Radar Demonstrator. 4

1.3 Frequency bands allocated to terrestrial broadcasting services designed by the ITU. . . . . . . . . . . . . . . . . . 5

2.1 Block diagram of a FMCW radar system . . . . . . . . . . . . . . . . 16

$2.2 \quad$ Linear frequency modulated waveform and its corresponding received signal. 16

2.3 Typical spectrum of a down-converted FMCW signal $\left(s_{r}(t) s_{t}(t)\right)$ with a linear chirp and the beat frequency. . . . . . . . . . . . . . . 17

2.4 Angle estimation using two RX antennas. . . . . . . . . . . . . . . . . . . 18

2.5 Angle estimation using four RX antennas. . . . . . . . . . . . . . . . . . . 19

2.6 Angle estimation using eight RX antennas. . . . . . . . . . . . . . . . . . 20

2.7 MIMO principle. . . . . . . . . . . . . . . . . . . 20

2.8 MIMO principle can be applied to two dimensional arrays. . . . . . . . . . 21

2.9 TDM concept. . . . . . . . . . . . . . . . . . 22

$3.1 \quad$ System Architecture of the presented MIMO Radar. . . . . . . . . . . . . . 26

3.2 Schematic representation of the MIMO antenna configuration with the physical array (left) showing the 24 TX antennas in blue, the 24 RX antennas in

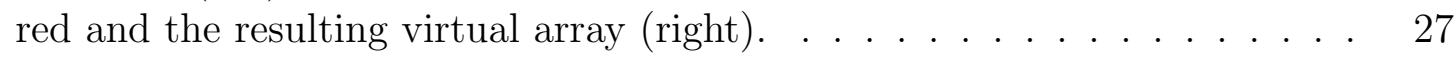

3.3 The graph illustrates the virtual array pattern with the designed maximum beamsteering of $\theta_{0}=25^{\circ}$ in red, the single element in blue and the resultant multiplication of both in black. The patterns are displayed in polar (left) and cartesian (right) coordinates. . . . . . . . . . . . . . 28

3.4 Sketch of the RF system. . . . . . . . . . . . . . . . . . . . . . . . . . . . . 29

3.5 Representation of a generic building block. . . . . . . . . . . . . . . . . . . 30

$3.6 \quad$ Representation of the used layer stack-up. The FR4 layers are milled where the TSA are located in order to enable the proper radiation of the antennas. 31

$3.7 \quad$ Fotography of the Calibration kit with different connectors. . . . . . . . . . 32

3.8 Model of microstrip line in AWR. . . . . . . . . . . . . . . . . . . . 32 
3.9 Comparison of the $S_{21}$ of between three $50 \Omega$ lines of different length $(25 \mathrm{~mm}$, $50 \mathrm{~mm}$ and $100 \mathrm{~mm}$ ) and its respective $S_{21}$ simulation produced with the presented model. . . . . . . . . . . . . . . . . . 33

3.10 Phase comparison of the phase of the $S_{21}$ of three different lines (with length of $100 \mathrm{~mm} 50 \mathrm{~mm}$ and $25 \mathrm{~mm}$ ) and their simulation with the presented model 34

3.11 Photography of the measured test amplifiers, HMC451 (left) and AVA-24+ (right).

3.12 Measured S-parameters of the test amplifiers, HMC451 (left) and AVA-24+

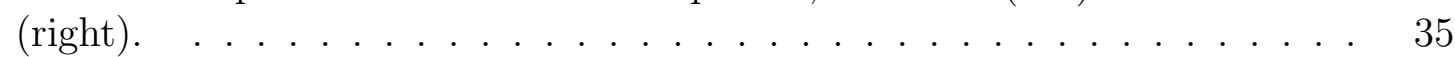

3.13 Photography of the building blocks. A 1-to4 Wilkinson divider (left) and 1-to-2 Wilkinson (right). . . . . . . . . . . . . . . . . . . 36

3.14 Measuremed S-parameters of 1-to-2 Wilkinson power divisor (left) adn 1-to4 power divisor (right). . . . . . . . . . . . . . . . . . . . . . 37

3.15 Photography of the selected switch connected to microstrip lines. . . . . . 38

3.16 Measured S-parameter of the built MASW-008322 GaAs SPDT switch. . . 39

3.17 System block diagram used for simulation purposes (left-below) and estimated system power levels (right-above). . . . . . . . . . . . . . . 41

3.18 Photography of the fabricated LO distribution board. A. PCB without components. B. PCB integrated in the radar system. C. PCB with soldered

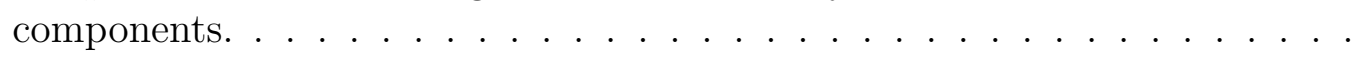

3.19 Comparison between the simulation and the measured results. For clarity reasons, only one path is depicted. It must be noted that the connectors are not included in the simulation. . . . . . . . . . . . . . . . .

3.20 A. Measurement of the amplitude of all 16 paths from the input to the output of the LO distribution board. B. Measurement of the phase of all 16 paths from the input to the output of the LO distribution board. C. Zoom at $17 \mathrm{GHz}$ of the measurement of the phase of all 16 paths from the input to the output of the LO distribution board. . . . . . . . . . . . . . . . . 44

3.21 System block diagram used for simulation purposes (left-below) and estimated system power levels (right-above). . . . . . . . . . . . . 46

3.22 Photography of the fabricated transmitter board. . . . . . . . . . . . . . . 47

3.23 Comparison between the simulation and the measured results. It must be noted that the connectors are not included in the simulation. . . . . . . . . 48

3.24 Measured maximum coupling between RX and TX antennas (left). Measured input power versus output power in the transmitter board (right). The measurement was performed from the input to the coupler C1 before the antenna. The effects of the coupler are compensated. . . . . . . . . . .

3.25 Photographies of the stacked TX, RX and LO panels. The left image shows the complete $\mathrm{RF}$ front-end unit. The right image is a section view of its

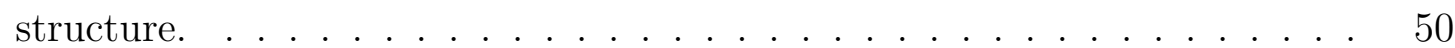

3.26 Photographies of the complete MIMO radar system. In the upper left image, the integrated RF front-end and digital system is presented. On the lower left and right images, a front view of the antenna array is shown. . . . . . . 50 
3.27 The MIMO radar test field with two corner reflectors at a range of $60 \mathrm{~m}$ with nominal radar cross section (RCS) of $36 \mathrm{~m}^{2}$ (left). Range-Azimuth section view of the 3D MIMO radar image capture from the radar setup scenario with two reflectors at a distance of $60 \mathrm{~m}$ (right). . . . . . . . . . . . . . . . 51

3.28 Angular separation capability test. On the left only one transmitter and traditional DBF is used. On the right, a MIMO algorithm is used. . . . . . 52

4.1 Block diagram of the 3D Imaging FMCW 16x16 MIMO RADAR Demonstrator. The upper and lower part of the image illustrate the RF front-end and the digital system blocks, respectively. . . . . . . . . . . . . . . . . 59

$4.2 \quad$ Schematic representation of the MIMO antenna configuration with the physical array (left) showing the 16 TX antennas in blue, the 16 RX antennas in red and the resulting virtual array (right). . . . . . . . . . . . . . 60

4.3 RX array in red, TX array in blue and virtual array in black. A unit in the graph is equivalent to $\frac{d_{x}}{2}$ and $\frac{d_{y}}{2}$ respectively. . . . . . . . . . . . . 61

4.4 Virtual array pattern. Single element in blue. MIMO array in red. Multiplication of both in black. In some graphs, the black line overlaps with the

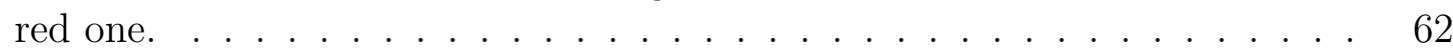

4.5 Schmatic diagram of the designed hardware. . . . . . . . . . . . . . . . . 63

4.6 Antenna stack-up (top). 3D view of the fabricated antenna with its main design parameters (bottom). . . . . . . . . ..... 65

4.7 A simulation of a typical Smith chart plot with the impedance locus versus frequency of a RSPA. . . . . . . . . . . . . . . . 67

4.8 Photography of the fabricated RSPA. . . . . . . . . . . . . . . . . . 68

4.9 Comparison between the measured of simulated $S_{11}$ of the single patch RSPA. 68

4.10 Comparison between the measured and simulated gain of the single patch

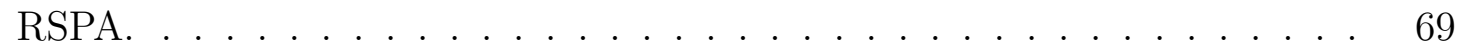

4.11 TX and RX layout. . . . . . . . . . . . . . . . . . . . . . . . . . . . . . . . . . . . . . .

4.12 A Sketch of the RX and TX feed networks. . . . . . . . . . . . . . . . . . . 71

4.13 Weights of amplitude of the RX antenna elements . . . . . . . . . . . . . . 72

4.14 Illustration of the simulated RX (bottom) and TX (top) feeds in a commercial software . . . . . . . . . . . . . . . . . 73

4.15 Feed of the RSPA TX and RX antenna arrays. . . . . . . . . . . . . . . . . 74

4.16 Gerber file of the milled cavities in the PCB in orange. . . . . . . . . . . . 74

4.17 Comparison between the simulated and measured matching of the RX and TX RSPA. . . . . . . . . . . . . . . . . 76

4.18 Comparison between the simulated and measured gain of the RX and TX

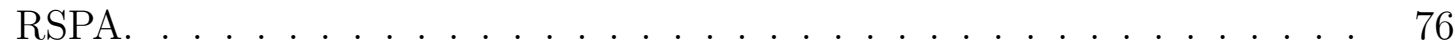

4.19 3D model of the fabricated vertical transition (top). Photography of the fabricated device (bottom). $\quad$. . . . . . . . . . . . . . . . . . . . 77

4.20 Comparison between simulated and measured S-parameter of the fabricated

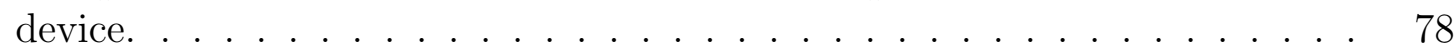


4.21 3-way Wilkinson divider. Delta configuration (left) and star configuration

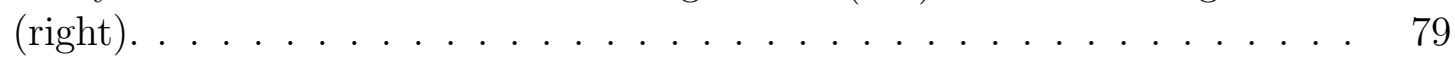

4.22 Image of the fabricated 3-way Wilkinson divider. Photography (left) and layout (right). . . . . . . . . . . . . . . . . . . . 80

4.23 Comparison between simulated and measured input reflection $\left(S_{11}\right)$ and transmission $\left(S_{12}, S_{13}\right.$ and $\left.S_{14}\right)$ S-parameters. $\ldots \ldots \ldots$. . . . . . . 80

4.24 Comparison between simulated and measured output reflection $\left(S_{22}, S_{33}\right.$ and

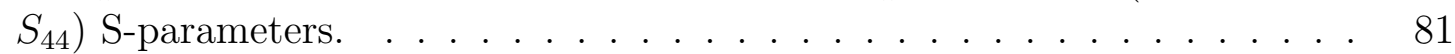

4.25 Comparison between simulated and measured isolation $\left(S_{23}, S_{34}\right.$ and $\left.S_{43}\right)$

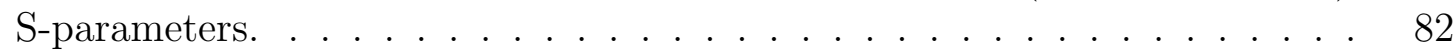

4.26 the chosen MIMO array configuration with its unoccupied space. . . . . . . 83

4.27 System block diagram used for simulation purposes (left-below) and estimated system power levels (right-above). . . . . . . . . . . . . 84

4.28 Photography of the frontend from the frontside. Patch antenna elements are only fairly visible, because they are covered by the upper substrate RO4003. The 4 Spiral antennas cannot be seen because they reside in an inner layer of the PCB. . . . . . . . . . . . . . . . . 85

4.29 Photography of the frontend from the backside. . . . . . . . . . . . . 85

4.30 Matching of the radar and communication antennas with the specification mask. ....................... 86

$4.31 S_{21}$ of the test set up described in text. The red line describes the theoretical value calculated with the Friis equation. . . . . . . . . . . . . . . 87

4.32 Picture of the 3D printed housing with antenna board and camera mounted in the middle. The markers show where the TX and RX antennas, and the camera are placed. . . . . . . . . . . . . . . . . . . 88

4.33 Azimuth and elevation profiles for a central target. . . . . . . . . . . . . 89

4.34 Normalized dependence of the received power in the elevation direction with the angle due to the antenna element pattern. . . . . . . . . . . . . . . . . 90

4.35 Normalized dependence of the received power in the azimuth direction with the angle due to the antenna element pattern. . . . . . . . . . . . . . . . 91

4.36 Image of the scene with three corner cubes marked with red circles. . . . . 92

4.37 3D reconstruction of the scene with radar data. The three targets are spaced in range and have different positions in azimuth and elevation. . . . . . . . 93

4.38 Camera and radar image overlaid. The color and the level of opaqueness is proportional to the amplitude of the reflection. The targets' ranges are displayed in boxes with different colors. The field of view is limited by the

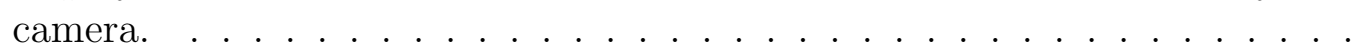

5.1 Circuit model of a traditional Wilkinson divider. . . . . . . . . . . . . . . . 100

5.2 Circuit model of a tapered Wilkinson divider. . . . . . . . . . . . . . . . . 101

5.3 Comparison between the simulated taper with and without pads. . . . . . 102

5.4 Even mode equivalent circuit model. . . . . . . . . . . . . . . . . . 103

5.5 Odd mode equivalent circuit model with $\mathrm{N}$ resistors. . . . . . . . . . . . . . 103 
$5.6 \quad$ Flow chart of the design method. . . . . . . . . . . . . . . . . . 105

5.7 Photography of the fabricated device. . . . . . . . . . . . . . . . . 106

5.8 Comparison between measured and simulated $S_{11}$ and $S_{21}$. The figure also shows the specification's mask. . . . . . . . . . . . . . . . . . . 107

5.9 Comparison between measured and simulated $S_{22}$ and $S_{32}$. The figure also shows the specification's mask. . . . . . . . . . . . . . . 107

5.10 Sketch of the proposed antenna. A. General view. B. Focus on the transition. C. Bottom view. D. Sideways view. . . . . . . . . . . . . . . 110

5.11 Photography of the back-to-back transition on the PCB with the main ge-

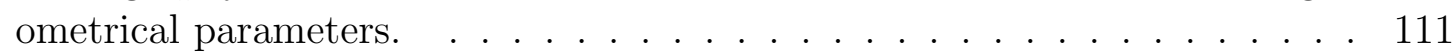

5.12 Simulated and measured S-parameters of the back-to-back PCB transition. 112

5.13 3D sketch of the MSL-to-PPW transition on the PCB. A. Main geometrical parameters. B. General view. . . . . . . . . . . . . . . . . . . 112

5.14 Picture of the back-to-back transition without $90^{\circ}$ bends. A. Screws. B. Sideways view showing $L_{p p w}$ and $w_{p p w 2}$. C. Unscrewed parts showing the critical face for RF. D. Microscope view showing $S_{\text {gap }}$. E. General view of the supporting legs. . . . . . . . . . . . . . . . . . 113

5.15 Simulated and measured S-parameters of the back-to-back transition with-

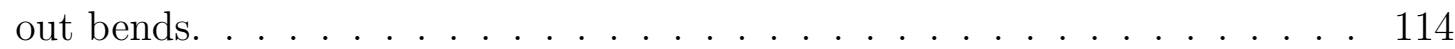

5.16 Simulated absolute value of the E-Field at $1.5 \mathrm{GHz}, 27 \mathrm{GHz}$ and $12 \mathrm{GHz}$. The red circles indicate the supporting legs which exite resonant modes at $1.5 \mathrm{GHz}$ and $27 \mathrm{GHz}$. The green circle indicates the supporting legs which do not excite resonant modes at 12 GHz. . . . . . . . . . . . . . 114

5.17 Back to back transition with $90^{\circ}$ bends. A. General view. B. Microscope

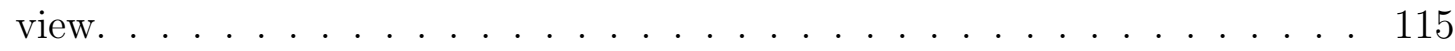

5.18 Simulated and measured S-parameters of the transition with bends. . . . . 115

5.19 Photography of the final 3D Printed Antenna. . . . . . . . . . . . . 116

5.20 Simulated and measured $S_{11}$ of the antennas with $90^{\circ}$ bend. . . . . . . 117

5.21 Simulated and measured gain of the antennas with and without bend. . . . 117

$6.1 \quad$ Layout of the designed antena with its main parameters. . . . . . . . . . . 124

6.2 Cross section view of the selected layer stack-up. . . . . . . . . . . . . . . . 124

6.3 A. Separated layers of the fabricated device. B. Stacked layers of the fabricated device. . . . . . . . . . . . . . . . . . . . . . . 126

6.4 Measurement of the fabricated Antenna. . . . . . . . . . . . . . . 126

6.5 Measured 3D gain. . . . . . . . . . . . . . . . . . . . . . . . . 127

6.6 Comparison between simulation and measurement of the H-plane radiation pattern of the fabricated antenna. . . . . . . . . . . . . . . 127

6.7 Comparison between simulation and measurement of the E-plane radiation pattern of the fabricated antenna. . . . . . . . . . . . . . . . . . 128

6.8 Representation of a waveguide Cross-guide coupler. . . . . . . . . . . . . . 129

6.9 Layer stackup of the designed $\mathrm{PCB} \ldots \ldots \ldots \ldots$ 
6.10 Photography of the separated subtrate layers. The layers are numbered corresponding to the substrate layers of Fig. 6.9 . . . . . . . . . . . . . . . 131

6.11 Photography of the mounted device. . . . . . . . . . . . . . . . . 131

6.12 Comparison between measured and simulated Transmission $\left(\left|S_{21}\right|\right)$. . . . . 133

6.13 Comparison between measured and simulated Return $\operatorname{loss}\left(\left|S_{11}\right|\right)$. . . . . . 133

6.14 Comparison between measured and simulated Coupling $\left(\left|S_{31}\right|\right)$. . . . . . . 134

6.15 Comparison between measured and simulated Isolation $\left(\left|S_{41}\right|\right)$. . . . . . . 134

6.16 Calculated Directivity $\left(D=\left|S_{31}\right|-\left|S_{41}\right|\right) . \ldots \ldots$. . . . . . . . . 135 


\section{List of Tables}

$3.1 \quad$ Description of main parameters of the calibration kit . . . . . . . . . . . . 31

3.2 Measured values of all 16 paths at $17 \mathrm{GHz}$. . . . . . . . . . . . . . . 45

$4.1 \quad$ Feed network impedances and its corresponding micorstrip line widths. . . 73

\begin{tabular}{|lll|l|l|}
\hline 4.2 & New antenna parameters of the RX and TX antennas. Fig. & 4.6 & illustrates \\
\hline
\end{tabular} an image with a description of the parameters. . . . . . . . . . . . . . 75

5.1 Position and value of the resistors . . . . . . . . . . . . . . . . . . 106

5.2 Comparative results of main parameters withother published results. . . . 108

5.3 Summary of time complexity . . . . . . . . . . . . . . . . 108

6.1 Design parameters of the fabricated device. . . . . . . . . . . . . . . . . . 125

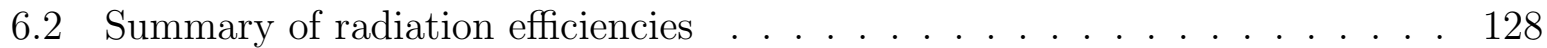

6.3 Specifications of the designed coupler . . . . . . . . . . . . . . . . . . . . 132

6.4 Comparison of broadband cross-guide couplers in planar form. . . . . . . . 135 


\section{Chapter 1}

\section{Introduction}

\subsection{Motivation}

A radar is a system [1] which uses radio waves and is capable of detecting the position and velocity of certain objects. In secrecy, eight different nations simultaneously developed systems of this type during the period from 1934 to 1939: Union of Soviet Socialist Republics (USSR), United Kingdom (UK), United States of America (USA), Japan, Italy, Germany, France and the Netherlands. Moreover, the USA and the UK shared their know how with some members of the Commonwealth: Australia, Canada, New Zealand, and South Africa, countries that also developed their own radar systems. In 1939 the term RADAR (RAdio Detection And Ranging) was established. The fast development of radar systems was probably one of the most important factors for the victory of the Allies. One example is the cavity magnetron designed by John Randall and Harry Boot in 1940 at the University of Birmingham (England). It was able to produce microwave power of kilowatts on higher frequencies. The high power signals of this device allowed the Allies to detect smaller objects from smaller antennas and reduced severely the radar's size. In such a manner, the second generation of radars could be integrated in fighter aircrafts or escort ships.

After the war, the politically unstable situation generated continuous radar improvements for military applications, especially in the USA, UK and USSR. Actually, these three nations made efforts in attracting german scientists and engineers to work on their new weapons (in the US it happened under Operation Paperclip). Apart from the military applications, the radar was used in numerous fields including marine navigation, civil aviation, meteorology and even medicine.

In the early 1950s, four techniques were developed and matured: the pulse-Doppler radar, which uses the Doppler-shifted signals from targets to detect their velocities; the monopulse radar, which improves the tracking accuracy; the phased-array radar, which enables the redirection of the main beam from one point to another very fast and the 
synthetic-aperture radar (SAR) with a single antenna in a moving platform (as an aircraft or a satellite), which combines the reflected waves of different pulses in different locations to produce a high-resolution image.

From the 1950s until now, a lot of civil applications of radar appeared. The development of the Air Traffic Control (ATC) increased the safety on passengers of civil flights, therefore it became mandatory for aircrafts flying in certain areas in 1960. Another civil application was the meteorology. The weather radar localizes the precipitation, calculates its velocity and direction and estimates its nature (ice, water snow...). In addition, radar astronomy enabled to make observations of extraterrestrial objects. The military industry also took advantage of the modern radars, notably for surveillance, for instance mounted in satellites, and military air, sea and land navigation.

Nowadays, the applications of radars are countless, millimeter wave scanner for security in airports [2], gesture recognition for modern electronic devices [3], surveillance of wide zones [4] and ecological studies [5] among others. In the global digitalization era, in which we are living, the integration of sensors with any kind of device as an autonomous vehicle, a smart-phone or augmented reality glasses, is playing a major role. In this scenario a multi-band Multiple Input Multiple Output (MIMO) radar is highly demanded mainly due to three reasons: It reduces conventional radar size and fabrication costs, it is capable of working worldwide without hardware design modifications and it combines different functionalities in a single and unique system.

\subsection{State of the Art}

Imaging radar systems are utilized for detection, tracking and classification of targets in a wide range of applications, thanks to their all-weather capabilities, material penetration properties and usability at day and night. Different imaging radar solutions exist. Mechanically steered radars are usually complex systems because of their physically moving components and long-term reliability is often compromised. Synthetic aperture radars (SAR) are a good solution that uses the motion of the radar antenna over a targeted region [6]. This leads to a finer spatial resolution, but there is a need for a continuously moving platform. Classical phased array radars require a high number of elements along the transmit and receive paths in order to achieve a high resolution [7]. For this reason, it tends to be a relatively large, complex and expensive approach.

Better performance can be achieved by utilizing a MIMO architecture [8]. In a MIMO system, orthogonal signals are emitted from the transmit antennas and jointly processed at the receivers. The signal orthogonality can, for example, be established in time-domain by employing a Time Domain Multiplexing (TDM) scheme switching between the transmit antenna elements in a round-robin fashion. In particular, this allows the calculation of 
amplitude and phase relationships of a large number of points in space, which is the result of multiplication of the number of transmit by the number of receive elements. These points form the elements of the virtual antenna array.
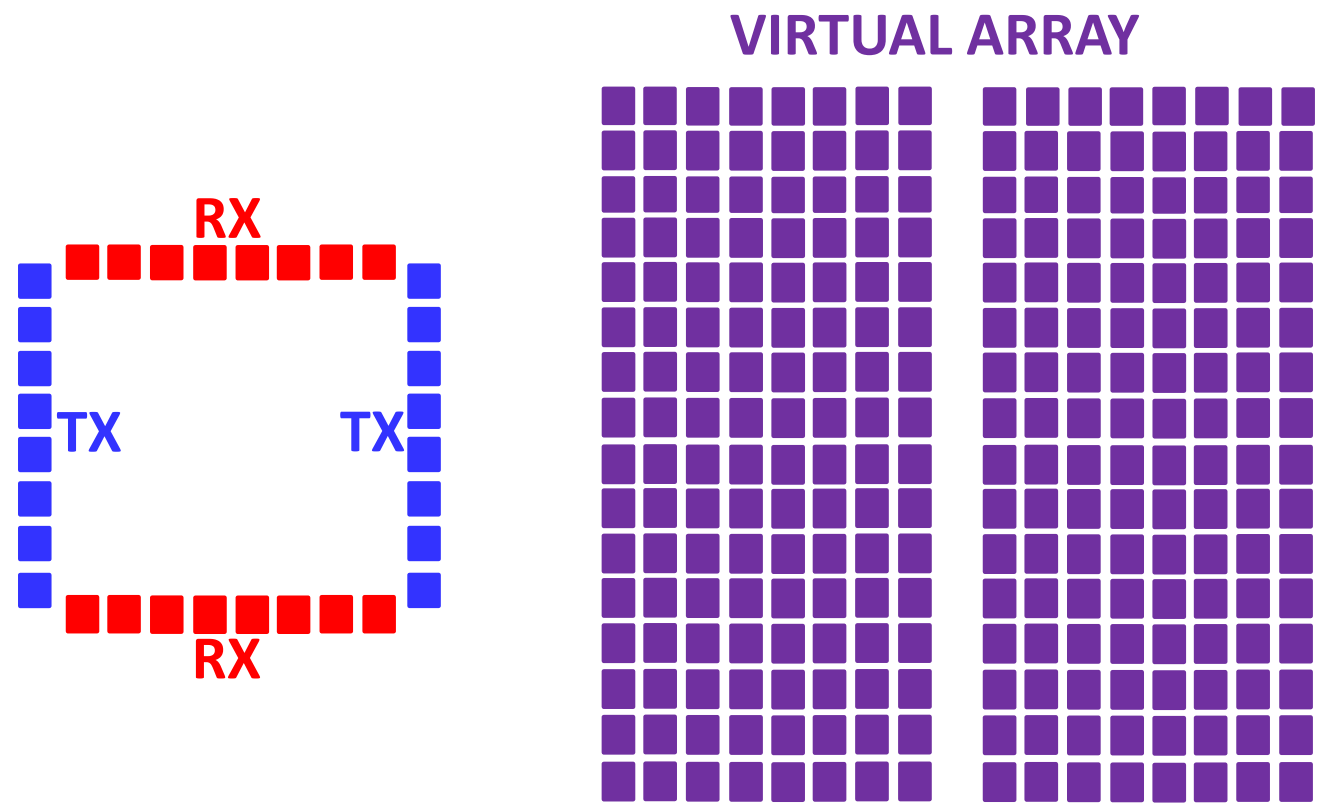

Figure 1.1: Schematic representation of the MIMO antenna configuration with the physical array (left) showing the $16 \mathrm{TX}$ antennas in blue, the $16 \mathrm{RX}$ antennas in red and the resulting virtual array (right).

The MIMO principle together with an appropriate antenna array arrangement achieves an improvement of the cross-resolution in comparison with a traditional phased array approach by an artificial increase of the antenna aperture with the so called virtual array concept. Therefore, in combination with a broadband signal, which determines the resulting resolution in the range direction, high-resolution 3D images of the captured scenario can be achieved in a smaller sized radar. Fig. 3.2 illustrates a MIMO physical array in which RX, TX and virtual elements are represented in red, blue and purple colors respectively.

Possible applications of 3D imaging MIMO radars for flying platforms are the situationawareness for rotorcraft as well as obstacle detection and terrain mapping in flight and critical landing operations. Furthermore, 3D MIMO radars can be used as sensor systems for medium-sized unmanned aerial vehicles (UAV) and for ground based surveillance of wide-zones and infrastructures. In the last years the scientific community has shown a growing interest in the MIMO radar topic. Hence, several MIMO radars have been pro- 
posed [9]-20] with very different performances and applications. However, the lack of a portable, medium range and multi-functional MIMO radar, characteristics that are within the scope of this work, was still present in the bibliography. The system architecture of a MIMO radar system is presented in Fig. 1.2. It is mainly divided into two parts: the RF frontend and the digital system. A Frequency Modulated Continuous Wave (FMCW) [21] signal is sent to a distribution board which provides the transmitters and the receivers. The sent signals reflect in the targets and return to the system through the receiver antennas to the received boards. There they will be mixed in order to generate the so called "beat frequency", which provides the information about the target.

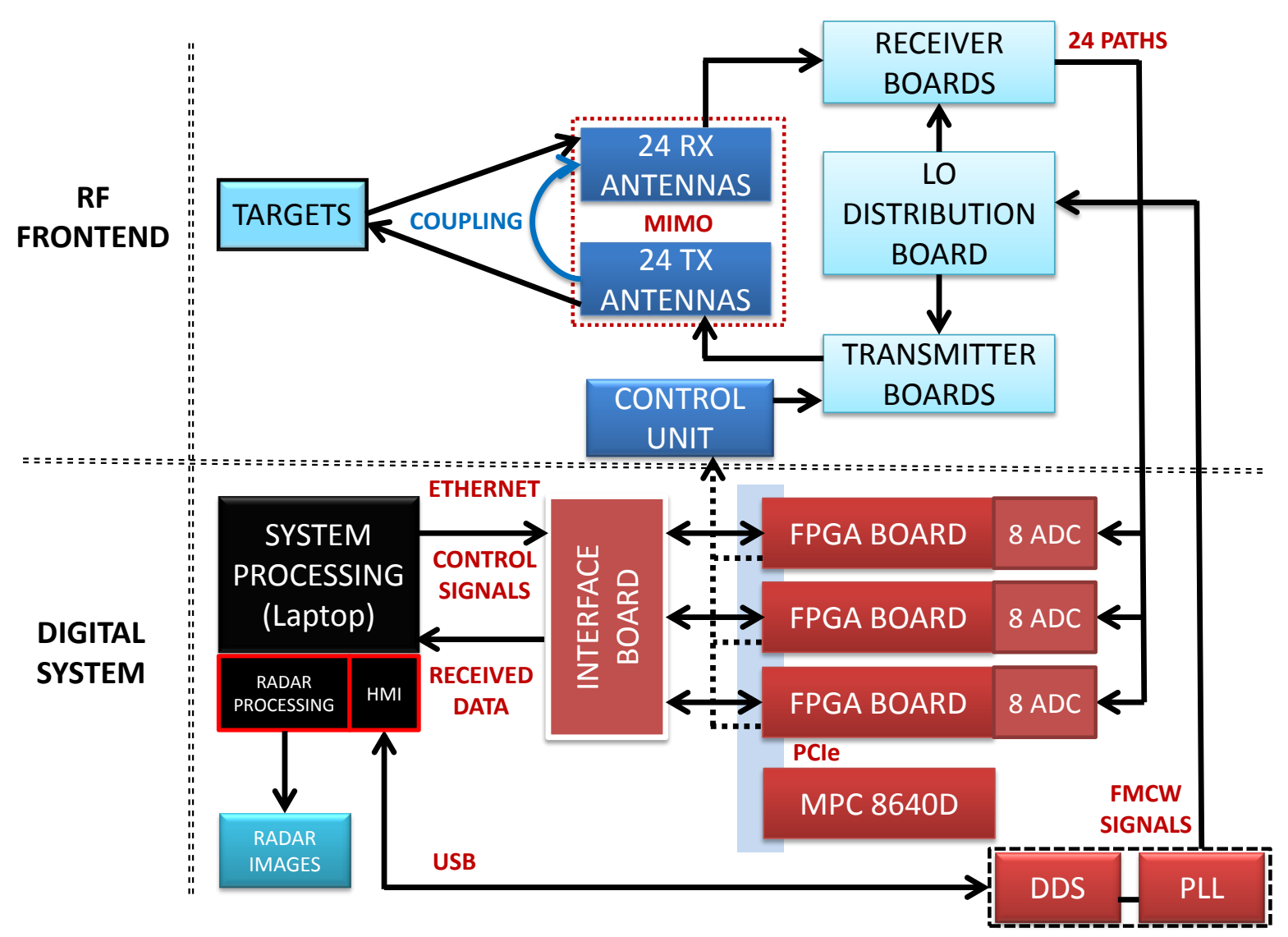

Figure 1.2: Block diagram of a 3D Imaging FMCW $24 x 24$ MIMO Radar Demonstrator.

Multifunctional systems which contain different functionalities within their specific frequency bands are highly desired, notably in the aeronautic industry [1], because they provide size, weight and power $(\mathrm{SWaP})$ reduction. There are two possibilities of combining these systems: an integration of different RF-frontends for each application or a single multi-band and multifunction RF-frontend which covers the needed part of the electromagnetic spectrum. The main advantage of the first approach is that all RF-functionalities can work simultaneously without interfering each other. 
MIMO Radar is a good solution for the sensor in terms of integration, cost and performance. The angular resolution increases considerably while the size, weight and number of TX/RX modules are reduced, if the virtual array is compared with a fully populated array [23] [24]. In addition, if MIMO TX and RX radiating elements are placed in a rectangular configuration, as Fig. 3.2 depicts, the center of the antenna aperture is not occupied and therefore available for different functionalities. In this unoccupied space a communication system, a torch, a gun, a radar jammer or camera, can be included, in such a way that the radar and the extra device share the same coordinate system and are integrated in the same system without increasing its size.

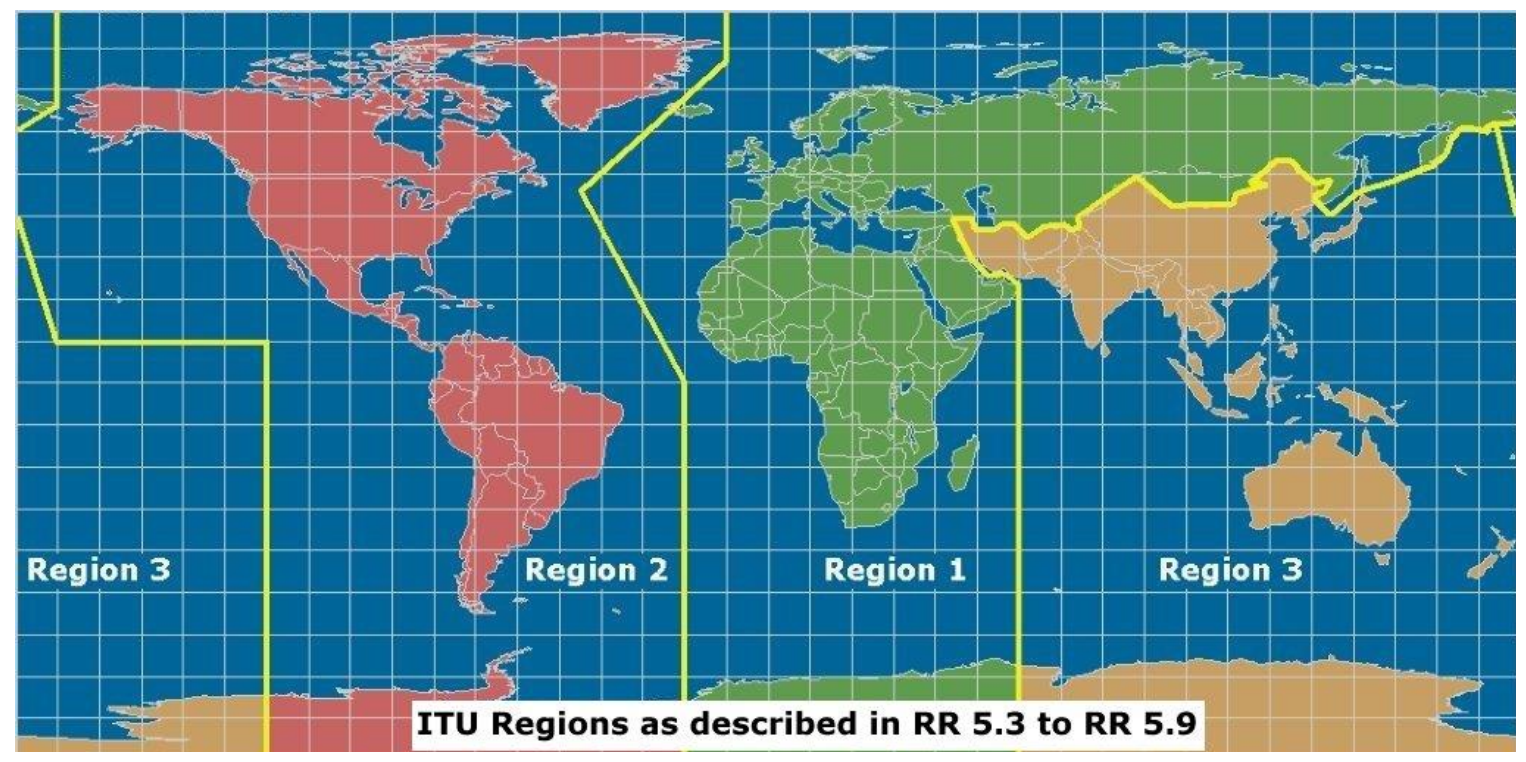

Figure 1.3: Frequency bands allocated to terrestrial broadcasting services designed by the ITU.

The advancing technological development goes hand in hand with the globalization and standardization of society and its demands. In this fast moving scenario a single product working in the whole world results extremely interesting due to its cost reduction capability, its fast implementation and its ability to remove unnecessary duplicities. The International Telecommunication Union (ITU) divided the world into three regions with different frequency bands allocated to terrestrial broadcasting services (see Fig. 1.3.). Usually, a radar product must be developed at least three times according to the frequency regulation. A broadband radar which covers the desired frequency bands is a good solution to this problem. Moreover, a broadband radar itself has multiple advantages: a larger operational bandwidth of the signals usually leads to a higher range resolution and allows frequency hopping techniques [3]. Furthermore, if the power of the signal is distributed over a larger frequency band, the probability of intercepting the radar decreases [4]. 
The Ultra-wideband (UWB) frequency range covers a bandwidth comprised between 3.1 to $10.6 \mathrm{GHz}$. It is very popular due to its multiple applications, such as communications, imaging radar, medical treatments and high data rate transmission. Consequently, there is a rich scientific literature dealing with broadband systems [28] and key RF components [29] [30] [31]. One of the objectives of this work is to develop key RF components, which work in a larger frequency band, from 5 to $40 \mathrm{GHz}$. This challenging frequency range is only a starting point. Indeed, what is truly interesting to know are the tools, methods and strategies that enable this large operational bandwidth. Thus, this frequency range can even be enlarged by paying some costs as a bigger device or more accuracy in the fabrication process. Regarding the selected initial frequency, the size of some components (antennas for instance) is typically inversely proportional to the frequency of operation. Considering that at $f_{\text {initial }}=5 \mathrm{GHz}$ the wavelength is $\lambda=60 \mathrm{~mm}$, the selected initial frequency is a good starting point for the required size of the targeted range of applications. Respecting the final frequency, the accuracy of fabrication process plays a major role since higher frequencies are severely affected by the fabrication process. Furthermore measurement equipment over $40 \mathrm{GHz}$ is expensive.

3D printing is revolutionizing the industry [16] with many applications for different sectors, such as the medical [17, automotive [18], construction [19], and aeronautic [20]. Some of the benefits of 3D printing are fast prototyping capability, design flexibility, low material waste, no middleman (from the printer to the user) and the possibility of cost and weight reduction. Over the last years, the scientific community has paid attention to and spent effort on combining this new technology with Radio Frequency (RF) applications. Proof of that is the increasing number of recently published articles related to this topic as for example a 3-D printed patch antenna [21], a vertical transition on a multilayer printed substrate [22], a multilayer MicroStrip Line (MSL) and a dipole antenna [23], dielectric reflect arrays [24], a traditional horn antenna [25] and a lens-antenna [26]. Some of the referenced ideas and modern fabrication processes are used in this work in order to achieve the proposed goals.

\section{$1.3 \quad$ Objectives}

The objective of this dissertation consists of three milestones.

First, a portable and low cost MIMO radar realization is presented. The Radio Frequency (RF) components are fabricated in a conventional Printed Circuit Board (PCB). The RF subsystems are integrated in panels which make up the desired antenna array arrangement in a sandwich configuration. This gives a lot of flexibility from the hardware's side. The fabricated frontend is also integrated with a digital platform forming together a complete portable radar system. After the processing of the radar signal some sample radar images are displayed. 
After that a second iteration of the system is completed. In this step, the panels in sandwich configuration are replaced by a single PCB with Resonant Slot Patch Antennas (RSPA). In such a manner, the frontend is summarized in a single PCB. An unoccupied space at the center of the MIMO array is also filled with a different functionality as a communication system and a camera. The frontend is integrated with a Xilinx Zynq Z-7020 System on Chip (SoC), which combines an ARM Cortex-A9 dual-core processor with a FPGA. The complete radar system is integrated with a 3D printed metallic part. It acts with double functionality: housing and cooling.

Thirdly, very broadband (5 - $40 \mathrm{GHz}$ ) RF key building blocks and novel low losses components are explored for future radar concepts. A tapered Wilkinson divider, which replaces its $\lambda / 4$ arms by tapered transmission lines, is integrated in a well-known technology as PCB achieving good performance over the targeted bandwidth. A strategy to design this complex device is also included. Here, a novel antenna, which takes advantage of a new and revolutionary fabrication process (3D printing), is also introduced. This antenna radiates in the direction perpendicular to the $\mathrm{PCB}$, in such a way that the panels of the first radar are not needed and a 2 dimensional antenna array can be integrated into a single PCB. Moreover, a 3D printed housing can also include the 3D printed antennas in its body.

\subsection{Methodology}

At first, a bibliography review including the state-of-the-art is reported. Following, a sketch of the complete system, in which the main RF components are represented, is completed. After that, the design, fabrication and test of first building blocks: antennas, power divider and RF amplifiers are performed. The RF components are first analyzed with classical network theory, if it results appropriate. Later on, they are optimized with a commercial software, such as AWR or CST. The components on-chip as amplifiers are searched on commercial catalogs. Once the design is finished and the chips arrived, a layout for a $\mathrm{PCB}$ is described with AWR or Altium designer depending on the complexity of the PCB. This PCB is manufactured by an external company. When the PCB is fabricated and the components are mounted, the circuits are measured. A network analyzer, power supply, multimeter and, if needed, a infrared camera are used for the complete characterization of the circuits (S-parameters in time and frequency domain, voltages and currents, hot-spots localization). The measurements are included into intern technical documents, and if some of them are suitable for publication, they are incorporated in scientific papers for journals and conferences.

Secondly, accurate RF subsystems are outlined. Using the experience accumulated in the last step these subsystems are integrated on PCBs, which are described due to its complexity in Altium designer. This time a network analyzer, power supply, multimeter, 
infrared camera and power meter are used to execute more system related measurement as coupling between antennas, $1 \mathrm{~dB}$ compression point of the channel, power levels in different points of the chain, hots-pots (candidates for a cooling system) or S-parameters of the complete chain. These results are included into technical documents and they are used for creating scientific literature, if it is convenient.

Thirdly, these subsystems are assembled together generating the RF unit of the Radar, which is integrated with the digital unit. The radar is transported to the radar test range in order to capture reflections of a corner cube. From these captures a radar image is generated. An evaluation of the image and a comparison with a picture of the radar test range is performed.

The accumulated lessons learned during the process previously explained are used in order to iterate one more time over the three steps with a more compact and multi-functional radar, which takes advantage of the free space on the center of the MIMO array for a second functionality placing communication antennas and a camera. RSPA with a multilayer PCB concept with air-gaps cavities are used for this approach.

At the end, novel RF concepts and components are explored in order to reduce costs, increment the bandwidth of operation and diminish the fabrication complexity. The suitable results are included into patents (US EU and Germany), international conferences and journals. Finally, the PhD dissertation is written.

\subsection{Structure of the Work}

Chapter 1 covers the introduction. In chapter 2 some basic MIMO and FMCW radar theory is described. Chapter 3 presents a complete MIMO radar system. In chapter 4 a compact multifunctional MIMO radar, which takes advantage of its unoccupied space in its center for a second functionality, is introduced. Chapter 5 deals with novel very broadband ( 5 to $40 \mathrm{GHz}$ ) key components for radar frontends. Chapter 6 introduces novel Empty Substrate Integrated Waveguide (ESIW) devices.

This work has been developed in collaboration between Airbus Group Innovations (AGI) [43] and Universitat Politècnica de València (UPV) [44]. The mission of AGI is to create prototypes with novel technologies, which are of common interest for the core business units (civil aircraft, helicopters, defence and space). After the development of the prototypes, the business units adapt this common development to its boundary conditions. During the thesis, I held a RF engineer PhD researcher position at AGI settled in München (Germany). All of the copyright material generated in this work is property of Airbus Defece \& Space GmbH. 
Some of the results of this work have been publicly funded by an european project ZoneSec [4] and a german project Fast [45]. 


\section{Bibliography}

[1] [Online]. Available: https://en.wikipedia.org

[2] [Online]. Available: https://en.wikipedia.org/wiki/Millimeter_wave_scanner

[3] [Online]. Available: https://atap.google.com/soli/

[4] [Online]. Available: http://www.zonesec.eu/

[5] R.H. Waring, J. Way, E. R. Hunt, L. Morrissey, K. J. Ranson, J.F. Weishampel, R. Oren and S.E. Franklin "Imaging radar for ecosystem studies." Bioscience vol. 45 No. 10. Nov. 1995.

[6] M. Soumekh, "Synthetic aperture radar signal processing", New York, Wiley, 1999.

[7] E. Brookner, "Phased-array radars", Scientific American 252.2, pp. 94-102, 1985.

[8] J. Li and P. Stoica, "MIMO Radar Signal Processing", John Wiley \& Sons, Inc., 2008.

[9] S. S. Ahmed, A. Schiessl and L. Schmidt, "Novel fully electronic active real-time millimeter-wave imaging system based on a planar multistatic sparse array", in $M i$ crowave Symposium Digest (MTT), 2011 IEEE MTT-S International, pp. 1-4, 5-10 June 2011.

[10] W. Wiesbeck, L. Sit, M. Younis, T. Rommel, G. Krieger and A. Moreira, "Radar 2020: The future of radar systems", 2015 IEEE International Geoscience and Remote Sensing Symposium (IGARSS), Milan, 2015, pp. 188-191.

[11] S. S. Ahmed, F. Gumbmann, A. Schiessl, S. Methfessel, C. Maire, A. Cenanovic, O. Ostwald, C. Evers and L. P. Schmidt, "QPass - Quick Personnel Automatic Safe Screening for Security Enhancement of Passengers", in 6th Future Security Research Conference., Berlin, Germany, Sept. 2011.

[12] G. M. Brooker, "Understanding millimetre wave FMCW radars", in 1st international Conference on Sensing Technology, Palmerston North, New Zealand, Nov. 21-23, 2005.

[13] A. Stove, "Linear FMCW Radar Techniques", in Radar and Signal Processing, IEE Proceedings F, vol. 139, no. 5, pp. 343-350, Oct. 1992. 
[14] Y. Huang, P. V. Brennan, D. Patrick, I. Weller, P. Roberts, and K. Hughes, "FMCW based MIMO imaging radar for maritime navigation", in Progress In Electromagnetics Research, Vol. 115, 327-342, 2011.

[15] J. Klare, M. Weiss, O. Peters, A. R. Brenner and J. Ender, "ARTINO: A New High Resolution 3D Imaging Radar System on an Autonomous Airborne Platform", in 2006 IEEE International Symposium on Geoscience and Remote Sensing, pp. 3842-3845, Denver, CO, USA, 2006.

[16] J. Klare, O. Saalmann, H. Wilden, and A. R. Brenner, "First Experimental Results with the Imaging MIMO Radar MIRA-CLE X", in Synthetic Aperture Radar (EUSAR), 2010 8th European Conference on, pp. 1-4, 7-10 June 2010.

[17] W. Mayer, A. Gronau, W. Menzel and H. Leier, 'A Compact 24 GHz Sensor for BeamForming and Imaging,", in 2006 9th International Conference on Control, Automation, Robotics and Vision, Singapore, pp. 1-6, 5-8 Dec. 2006.

[18] M. Harter, A. Ziroff and T. Zwick, 'Three-dimensional radar imaging by digital beamforming,", in Radar Conference (EuRAD), Manchester, pp. 17-20, 12-14 Oct. 2011.

[19] D. Cerutti-Maori and J. Klare, "Coherent MIMO radar for GMTI", in Geoscience and Remote Sensing Symposium (IGARSS), 2010 IEEE International, pp.1085-1088, 25-30 July 2010.

[20] X. Zhuge and A. G. Yarovoy, "A Sparse Aperture MIMO-SAR-Based UWB Imaging System for Concealed Weapon Detection", in IEEE Transactions on Geoscience and Remote Sensing, vol. 49, no. 1, pp. 509-518, Jan. 2011.

[21] G. M. Brooker, "Understanding millimetre wave FMCW radars", in 1st international Conference on Sensing Technology, Palmerston North, New Zealand, Nov. 21-23, 2005.

[22] G.C. Tavik, C.L. Hilterbrick, J.B. Evins, J.J. Alter, J.G. Crnkovich, J.W. de Graaf, W. Habicht, G.P. Hrin, S.A. Lessin, D.C. Wu, and S.M. Hagewood, "The advanced multifunction RF concept", in IEEE Trans. Microw. Theory Techn., vol. 53, no. 3 pp. 1009-1020, Mar. 2005

[23] J. Klare, O. Saalmann, H. Wilden, A.R. Brenner, "First Experimental Results with the Imaging MIMO Radar MIRA-CLE X", in Synthetic Aperture Radar (EUSAR), 2010 8th European Conference, Singapore, vol., no., pp.1-4, 7-10 June 2010

[24] Y. Huang, P.V. Brennan, D. Patrick, I. Weller, P. Roberts and K. Hughes., "FMCW Based MIMO imaging radar for maritime navigation", in Progress In Electromagnetics Research, vol. 115, pp. 327-342, 2011

[25] [Online]. Available: http://life.itu.int/radioclub/rr/art05.htm 
[26] R. G. Wiley, "Electronic intelligence: The interception of radar signals", Artech House, 1985 - Technology \& Engineering - 284 pages

[27] M. Jankiramen, "Design of Multi-Frequency CW Radars", SciTech Publishing Inc, 2007 - 351 - ISBN-13:978-1-891121-56-2

[28] G.N. Saddik, R. Singh and E. Brown., "Ultra-wideband multifunctional communications/radar system", in IEEE Trans. Microw. Theory Techn., vol. 55, no. 7, pp. 1431-1437, Jul 2007

[29] C.T. Chiang and B.K. Chung. "Ultra wideband power divider using tapered line", in Progress In Electromagnetics Research, vol. 106, pp. 63-73, 2010

[30] L. Chang, C. Liao, L. L. Chen, W. B. Lin, X. Zheng and Y. L. Wu. "Design of an ultra-wideband power divider via the coarse-grained parallel micro-genetic algorithm", in Progress In Electromagnetics Research, vol. 124, pp. 425-440, 2012

[31] K. Shamaileh, M. Almalkawi, V. Devabhaktuni, N. Dib, B. Henin and A. Abbosh. "Non-uniform transmission line ultra-wideband wilkinson power divider", in Progress In Electromagnetics Research, vol. 44, pp. 1-11, 2013

[32] I.J. Petrick and T. W. Simpson "3D printing disrupts manufacturing: how economies of one create new rules of competition." Research-Technology Management, 2013 Taylor \& Francis.

[33] J.Zuniga, D.Katsavelis, J.Peck, J.Stollberg, M.Petrykowski, A.Carson and C. Fernandez "Cyborg beast: a low-cost 3d-printed prosthetic hand for children with upper-limb differences." BMC Research Notes 2014.

[34] [Online]. Available: https://localmotors.com/3d-printed-car/

[35] [Online]. Available: https://3dprint.com/tag/3d-printed-house/

[36] M.Tomlin, J.Meyer "Topology optimization of an additive layer manufactured (ALM) aerospace part." in The 7th Altair Technology Conference 2011.

[37] M.Liang, C.Shemelya, E. MacDonald, R.Wicker and H.Xin "3-D Printed Microwave Patch Antenna via Fused Deposition Method and Ultrasonic Wire Mesh Embedding Technique" in IEEE Antennas and Wireless Propag. Lett., vol. 14, pp.1346-1349 2015.

[38] M.Liang, X. Yu, C.Shemelya, E. MacDonald and H.Xin "3D Printed Multilayer MSL Structure with Vertical Transition toward Integrated Systems" in 2015 IEEE MTT-S International Microwave Symposium, Phoenix, AZ, 2015, pp. 1-4 .

[39] C.Shemelya, M.Zemba, M.Liang, D. Espalin, C.Kief, H.Xin, R.B. Wicker and E.W. MacDonald "3D PRINTING MULTI-FUNCTIONALITY. Embedded RF Antennas and Components" in 2015 9th European Conference on Antennas and Propagation (EuCAP), Lisbon, 2015, pp. 1-5 . 
[40] P. Nayeri, M. Liang, R. A. Sabory-Garcia, M. Tuo, F. Yang, M. Gehm, H.Xin, A. Z. Elsherbeni "3D Printed Dielectric Reflectarrays: Low-Cost High-Gain Antennas at Sub-Millimeter Waves" in IEEE Transactions on Antennas and Propagation, vol. 62, no. 4, pp. 2000-2008, April 2014.

[41] C.R. Garcia, R.C. Rumpf, H.H. Tsang and J.H. Barton "Effects of extreme surface roughness on 3D printed horn antenna" in ELECTRONICS LETTERS 6th June 2013 Vol. 49 No. 12.

[42] F. Gianesello, A. Bisognin, D. Titz, C. Luxey, C.A. Fernandes, J.R. Costa and D. Gloria "3D printing technology: Enabling innovative \& cost effective industrial antenna solution" in 2016 International Workshop on Antenna Technology (iWAT), Cocoa Beach, FL, 2016, pp. 55-56.

[43] [Online]. Available: http://www.airbusgroup.com/int/en/corporate-socialresponsibility/airbus-group-innovations.html

[44] [Online]. Available: https://www.upv.es

[45] [Online]. Available: http://en.fast-zwanzig20.de/verkehr/fast-imaging/ 


\section{Chapter 2}

\section{General Review of MIMO Radar}

\subsection{Overview}

The objective of this chapter is to establish a framework for understanding the benefits and disadvantages of the MIMO radar arquitecture. The term MIMO simply refers to multiple radiating and receiving elements. A MIMO radar transmits orthogonal waveforms, that usually are received simultaneously. There are different ways to achieve diversity in the signals. A good solution is to use OFDM signals, in which each transmitter antenna has dedicated a portion of the operational frequency band. Another popular approach is to utilize a TDM scheme, in which each signal is transmitted in a different time period and is then simultaneously received.

Once all possible combinations between TX and RX signals are captured, they are arranged by generating a virtual array which is larger than the physical. The position of the antennas in the virtual array can be obtained from the discrete convolution of the positions of the RX antennas and TX antennas. Thus, the position of the antennas is of vital importance in order to avoid different combinations of RX and TX which land in the same virtual element (redundant elements).

In this work a FMCW TDM scheme is used. For this reason, a brief introduction of these topics is also completed.

\subsection{Frequency Modulated Continuous Wave Radar}

The radars presented in this work are based on the Frequency Modulated Continuous Wave Radar (FMCW technique) [1] [2]. A way to measure a target's rage and/or speed is to transmit a signal with a frequency variation in time and to down mix this transmitted signal with the received one. In such a manner, the correlation of signals provides the beat frequency with the information of the target's range. Fig. 2.1 illustrates a basic block 
diagram of a FMCW radar system.

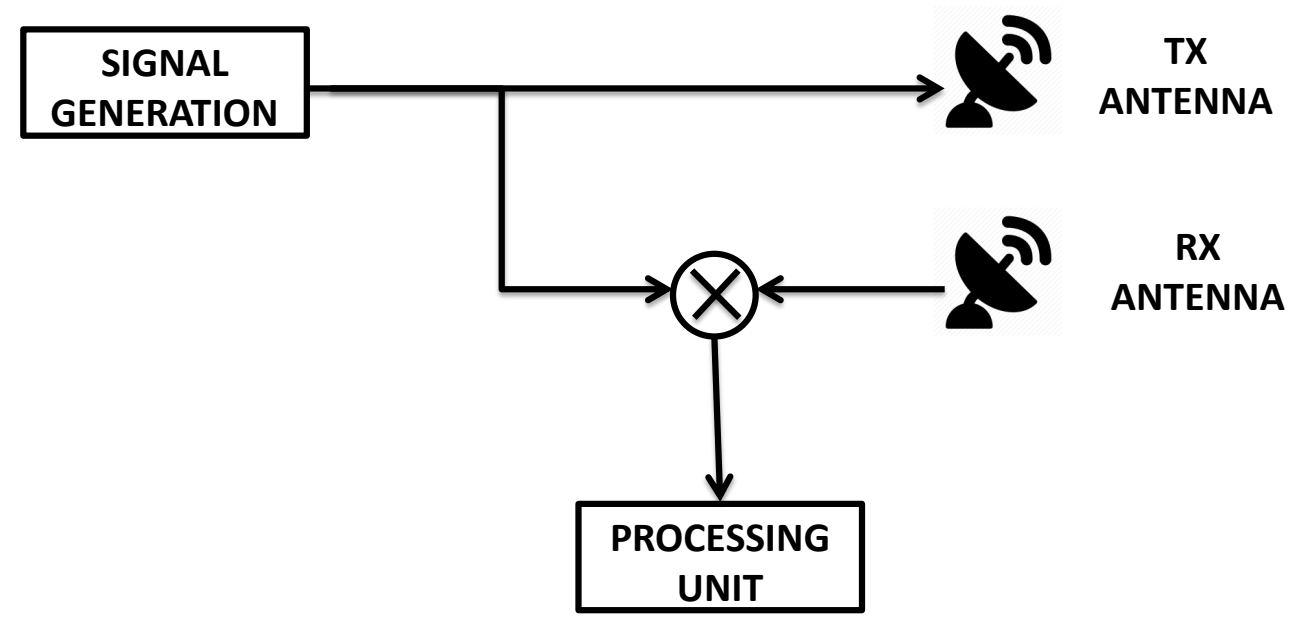

Figure 2.1: Block diagram of a FMCW radar system

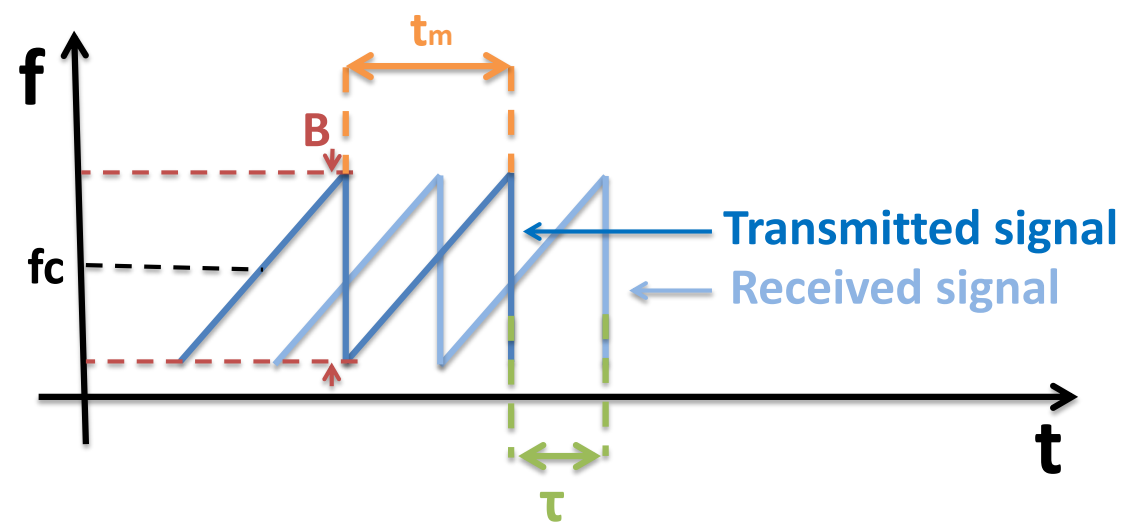

Figure 2.2: Linear frequency modulated waveform and its corresponding received signal.

Fig. 2.2 illustrates a linear frequency modulated waveform and the received signal. The frequency of the transmitted signal of a FMCW radar can be triangular as follows (2.1):

$$
f(t)=f_{c}+\frac{B}{t_{m}} t-\frac{B}{2}
$$


for $0<t<t_{m}$ and zero elsewhere, $B$ is the bandwidth of the transmitted signal, $t_{m}$ is the period of the transmitted signal, $f_{c}$ is the central frequency of the transmitted signal and $\tau$ is the time delay (which contains the information of the range of the target).

$$
\begin{gathered}
s_{t}(t)=A \cdot \sin \left\{2 \pi\left(f_{c}+\frac{B}{t_{m}} t-\frac{B}{2}\right) t\right\}=A \cdot \sin \left\{2 \pi\left(f_{c} t+\frac{B}{t_{m}} t^{2}-\frac{B}{2} t\right)\right\} \\
s_{r}(t)=A \cdot \sin \left\{2 \pi\left(f_{c}(t-\tau)+\frac{B}{t_{m}}(t-\tau)^{2}-\frac{B}{2}(t-\tau)\right)\right\}
\end{gathered}
$$

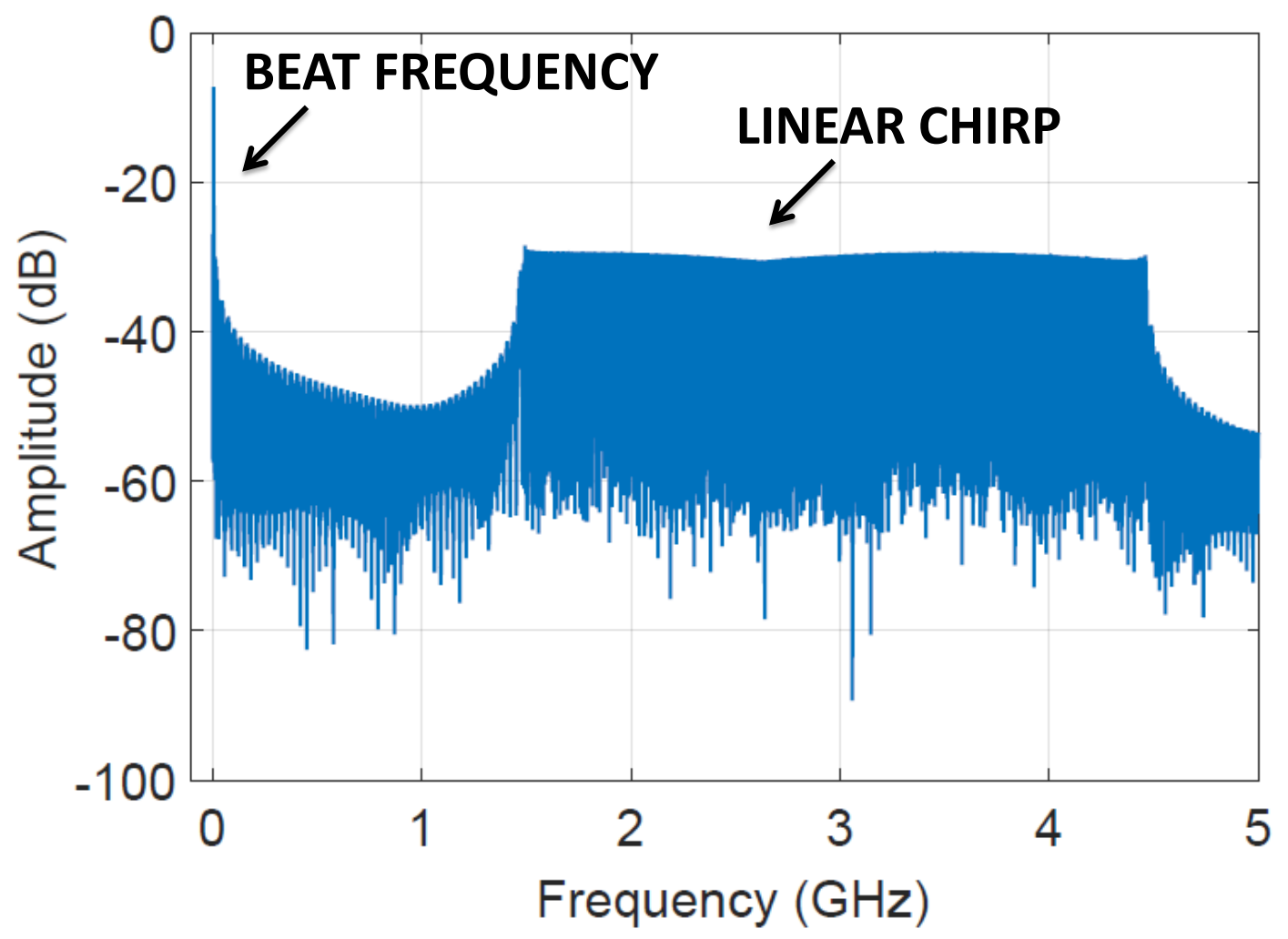

Figure 2.3: Typical spectrum of a down-converted FMCW signal $\left(s_{r}(t) s_{t}(t)\right)$ with a linear chirp and the beat frequency.

Equations (2.2) and (2.3) are the transmitted and received signals. When (2.2) and (2.3) are multiplied, there are two different parts of the spectrum clearly identifiable as Fig.2.3 illustrates. A linear chirp in higher frequencies (this part is not interesting for us) 
and the so called "beat frequency" which is linearly proportional to $\tau$ (2.4). Once $\tau$ is known, the range can be straightforward calculated with (2.5) in which $\mathrm{R}$ is the range of the target and $\mathrm{c}$ is the speed of the light.

$$
f_{\text {beat }}=\frac{B}{t_{m}} \tau
$$

$$
R=c \tau
$$

\subsection{Angle Estimation Basics}

In order to estimate the angle of arrival of a reflective object, at least two RX antennas are required. A sketch of a radar that has one TX and two RX, separated by a distance $d$, is displayed on Fig. 2.4 .

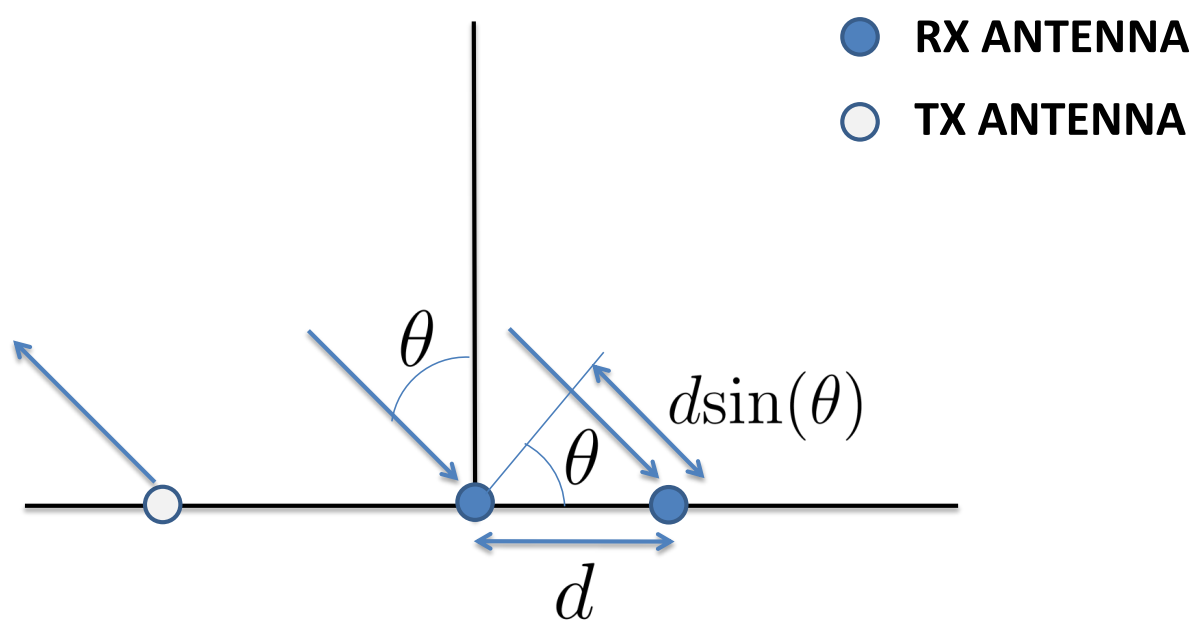

Figure 2.4: Angle estimation using two RX antennas.

The sent signal from the TX antenna reflects the target (at an angle $\theta$ with regard to the radar) and arrives at both RX antennas. The received signal must travel an additional distance of $d \sin (\theta)$ to reach the second antenna, this corresponds to phase difference of $\phi=(2 \pi \lambda) d \sin (\theta)$ between the signals. This can be used to calculate the angle of arrival as 
Eq. (2.6) shows.

$$
\theta= \pm \sin ^{-1}\left(\frac{\phi \lambda}{2 \pi d}\right)
$$

Since $\phi$ can be uniquely estimated only in the range $(-\pi, \pi)$, the unambiguous field of view (FOV) [3] of the radar is 2.7):

$$
\theta_{F O V}= \pm \sin ^{-1}\left(\frac{\lambda}{2 d}\right)
$$

In such a manner that the maximum FOV is achieved with $d=\lambda / 2$ as Eq. (2.8) shows.

$$
\theta_{F O V}= \pm 90^{\circ}
$$

Usually, a radar has more than two RX antennas. Fig. 2.5 illustrates the case of $N_{R X}=4$. Each additional antenna provides an additional phase-shift of $\phi$. Accordingly, a linear progression in the phase of the signal across the $\mathrm{N}$ antennas (in this case, $[0 \phi 2 \phi$ $3 \phi])$ occurs. Hence, $\phi$ can be estimated by sampling the signal across the $N_{R X}$ antennas, and performing an FFT on the signal sequence.

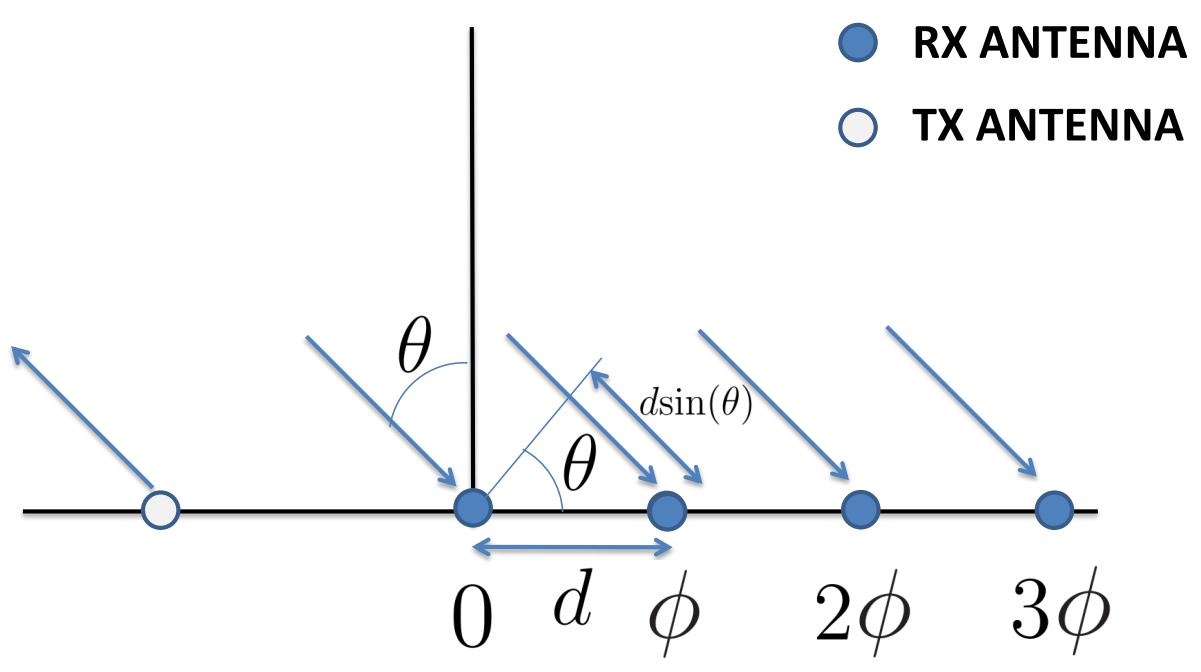

Figure 2.5: Angle estimation using four RX antennas.

The higher the number of antennas, the sharper the peak of the FFT results, in such a way that the accuracy of the angle estimation is improved. 


\subsection{Principle of the MIMO Radar}

Continuing with the example of the previous section, if the target is to double the angle resolution capability of the radar (Fig. 2.5), one solution is to double the number of RX antennas (from four to eight), as Fig. 2.6 displays.

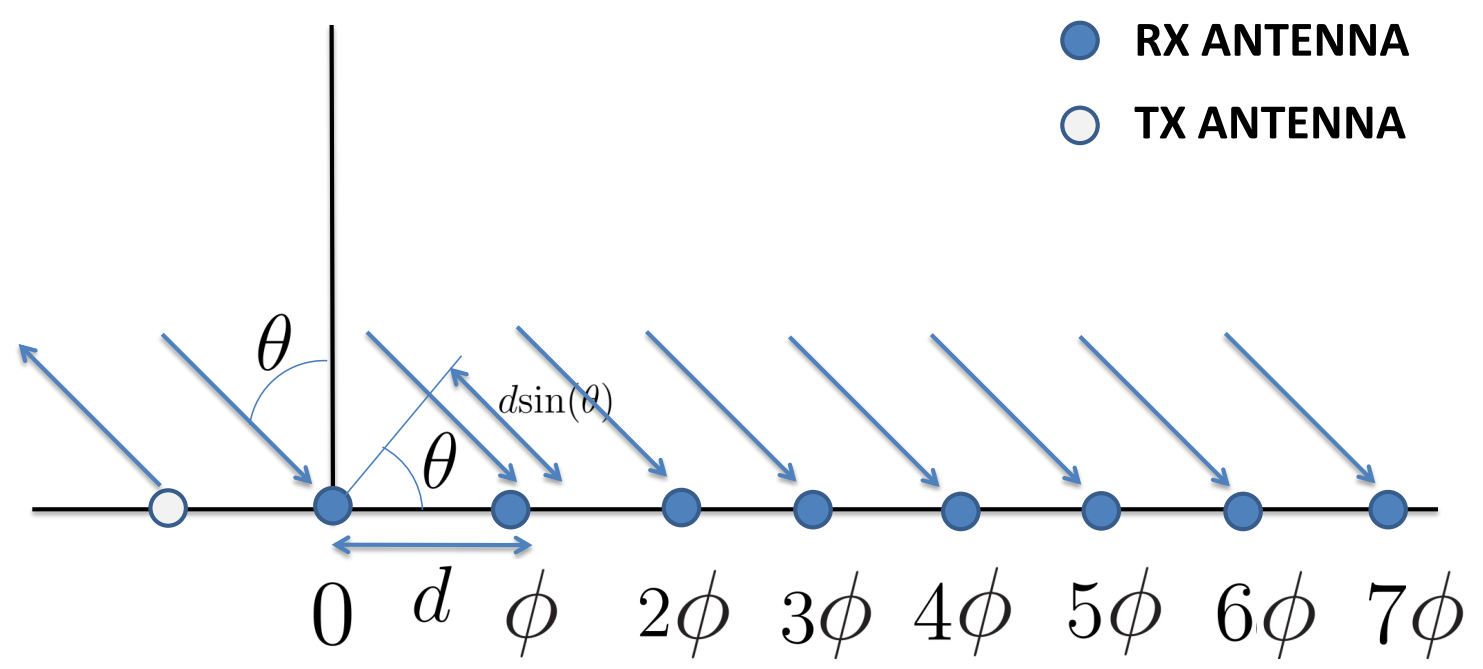

Figure 2.6: Angle estimation using eight RX antennas.

Using MIMO, the same result can be obtained by adding just a TX antenna.

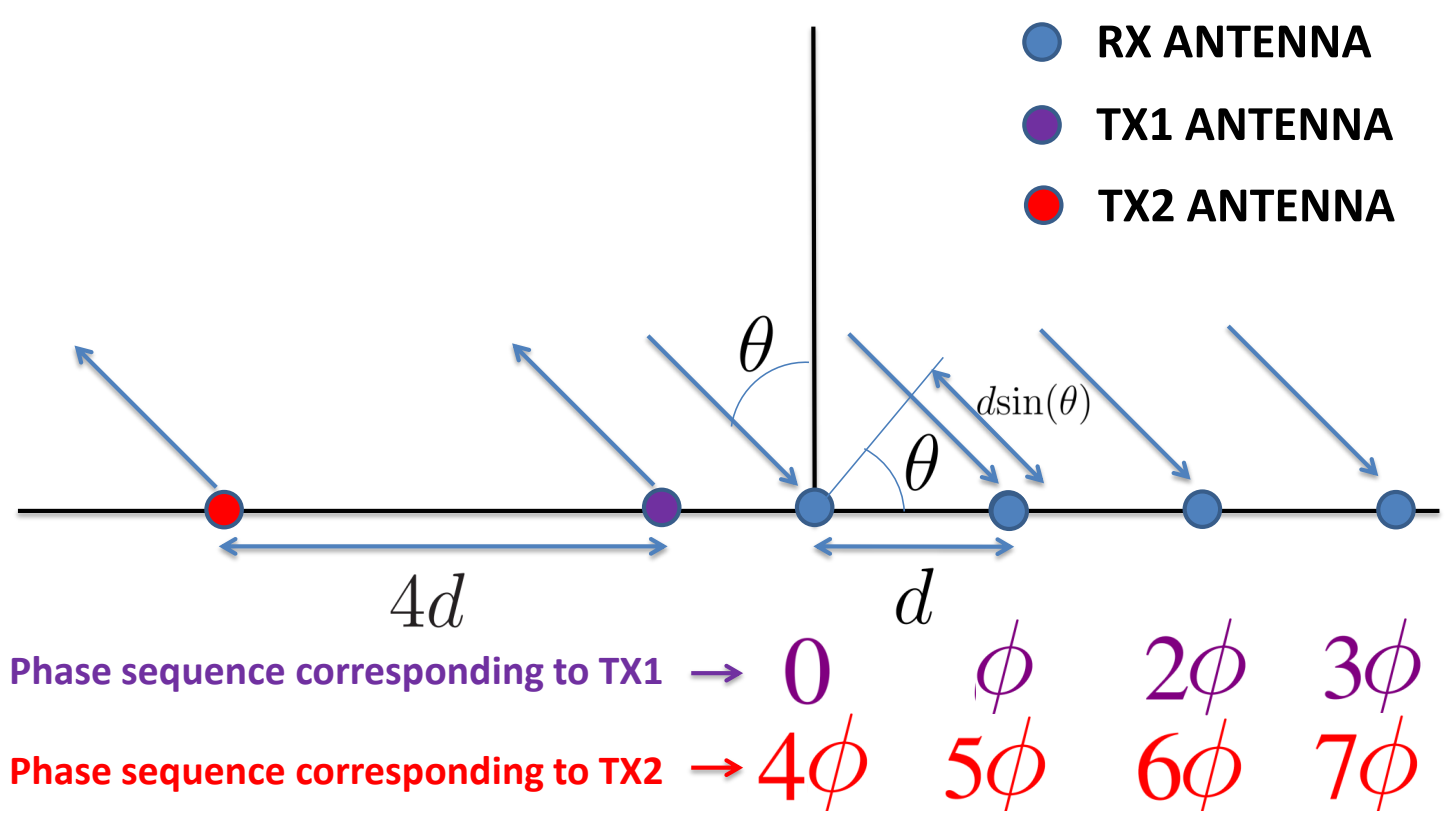

Figure 2.7: MIMO principle. 
Fig. 2.7 has two TX antennas, TX1 and TX2. The transmission from TX1 provides

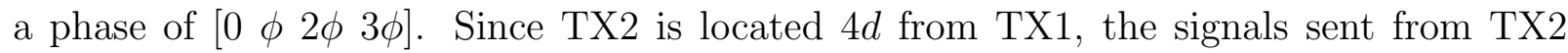
provide an additional path $4 d \sin (\theta)$ compared to TX1. Therefore, the signals at the RX antennas see an additional phase-shift offset of $4 \phi$. The phase sequence due to the TX2 antenna is $[4 \phi 5 \phi 6 \phi 7 \phi]$. By combinnig the TX1 and TX2 the same sequence that the configuration in Fig 2.6 can be reproduced, in such a manner that the configuration with two TX antennas synthesizes a virtual array of eight RX antennas.

In order to generalize the discussion, with $N_{T X}$ and $N_{R X}$ antennas placed in a proper position a virtual array of $N_{T X} \mathrm{X} N_{R X}$ can be generated. Hence, employing MIMO radar techniques results in a multiplicative improvement of the angle resolution.

If $p_{m}$ represents the coordinates of $m^{\text {th }} \mathrm{TX}$ antenna $\left(\mathrm{m}=0,1, \ldots N_{T X}\right)$, and $q_{n}$ represents the coordinates of the $n^{t h} \mathrm{RX}$ antenna $\left(\mathrm{n}=0,1, \ldots N_{R X}\right)$, then the position of the virtual antenna elements $\left(k_{i}\right)$ can be calculated as $p_{m}+q_{n}$, for all possible values of $\mathrm{m}$ and $\mathrm{n}$, which lead to the discrete convolution. For instance, in Fig. 2.7, $p_{1}=0$ and $p_{2}=4 d$, and $q_{1}=0, q_{2}=d, q_{3}=2 d$ and $q_{4}=3 d$ and the virtual array elements are located at $k_{1}=0$, $k_{2}=d, k_{3}=2 d, k_{4}=3 d, k_{5}=4 d, k_{6}=5 d, k_{7}=6 d$ and $k_{8}=7 d$.

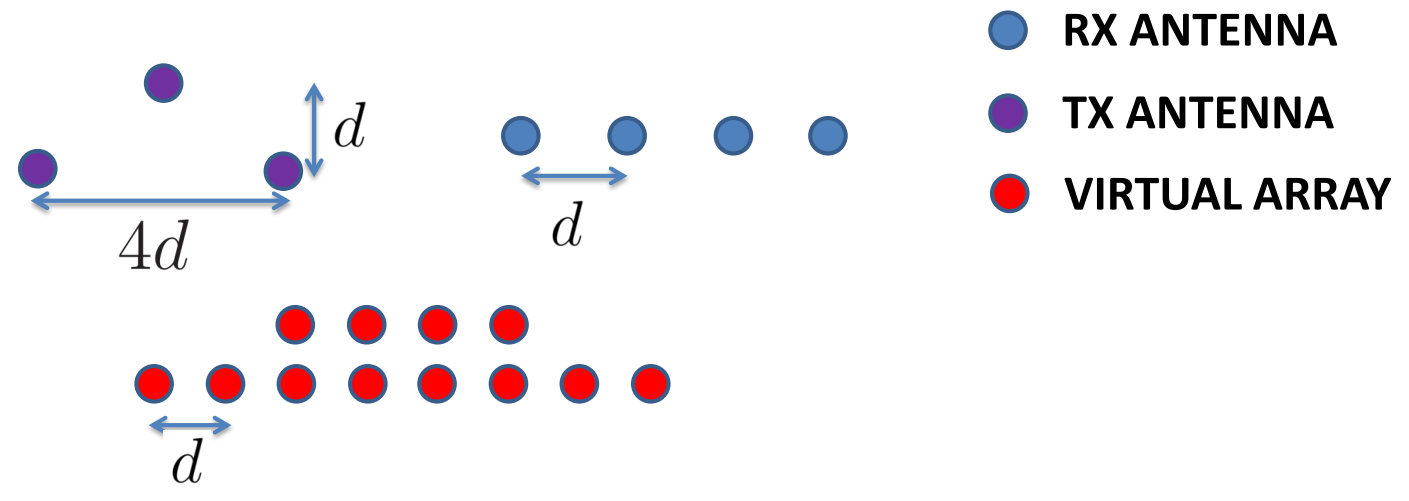

Figure 2.8: MIMO principle can be applied to two dimensional arrays.

Fig. 2.8 illustrates how the MIMO principle can also be extended to multidimensional arrays.

\subsection{Time Division Multiplexing}

The previous section introduced the basic mechanism of the MIMO technique applied to radar theory. It is important to note that the RX antennas must be able to separate the signal coming from the different TX in order to implement the MIMO technique. There are several ways to achieve it. In this work we have focused on Time Division Multiplexing (TDM), this method is described in the context of FMCW radars. 
In TDM - MIMO [4, the orthogonality is achieved in time. Each frame consists of several blocks, with each block consisting of $N_{T X}$ time slots correspoding to the TX antennas. Fig. 2.9 illustrates an example with $N_{T X}=2$.

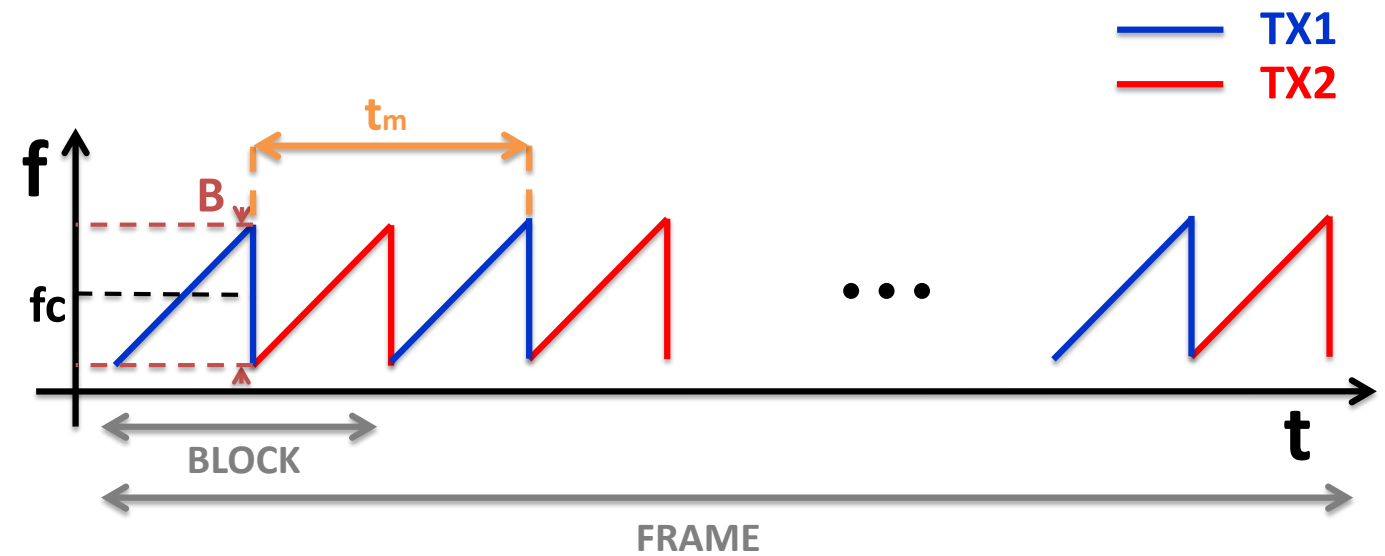

Figure 2.9: TDM concept.

In a TDM-MIMO FMCW radar scheme, a 2D FFT (range-Doppler FFT [5]) is performed in each TX-RX pair. Each of these pairs represents one virtual antenna. 


\section{Bibliography}

[1] G. M. Brooker, "Understanding Millimetre Wave FMCW Radaes", in 1st International Conference on Sensing Technology, November 21-23. 2005 Palmerston North, New Zealand.

[2] P. E. Pace, "Detecting and classifying low probability of intercept radar", Artech house, second edition, 2009.

[3] S. Rao, "MIMO Radar Application Repot", Texas Instruments, SWRA554 - May 2017.

[4] F. C. Robey, S. Coutts, D. Weikle, J. C. McHarg and K. Cuomo "MIMO Radar Theory and Experimental Results", in 38th Asimolar Conference on Signal, Systems and Computes, November 7-10. 2004 Pacific Grove, CA, USA.

[5] [Online]. Available: https://training.ti.com/mmwave-training-series 


\section{Chapter 3}

\section{A MIMO Radar Implementation}

In this chapter, the first implementation of a MIMO radar at Airbus Group innovations is presented. The main aim of this work is to give a first approach in order to verify the suitability for a future product. The objective is to achieve the Technology Readness Level (TRL) 4 defined by the National Aeronautics and Space Administration (NASA):" TRL 4: Component/subsystem validation in laboratory environment: Standalone prototyping implementation and test. Integration of technology elements. Experiments with full-scale problems or data sets." [1].

In the following sections a complete MIMO radar is described in order to give a complete idea about the system. However, my contributions are focused on measurement and integration of the system, design, layout routing and measurement of the LO Distribution board as well as design and measurement of the transmitter. As a consequence, a more detailed description of these parts is completed.

\subsection{System Architecture}

The top-level system structure of the built MIMO Radar demonstrator is presented in the form of a block diagram in Fig. 3.1. Here, the two main building blocks can be seen: the $\mathrm{RF}$ frontend and the digital system.

The RF front-end system is the essence of the transmit and receive signals analog operations. It consists of the transmit boards, the receive boards, the transmit and receive antenna arrays and the Local Oscillator (LO) distribution board. The comprehensive description of the RF frontend is given in following sections.

The signal processing is at the core of the digital system. It consists of a system processing on a laptop for radar control and radar signal processing, a combined Direct Digital Synthesizer (DDS) and Phase-Locked Loop (PLL) system for the generation of the config- 


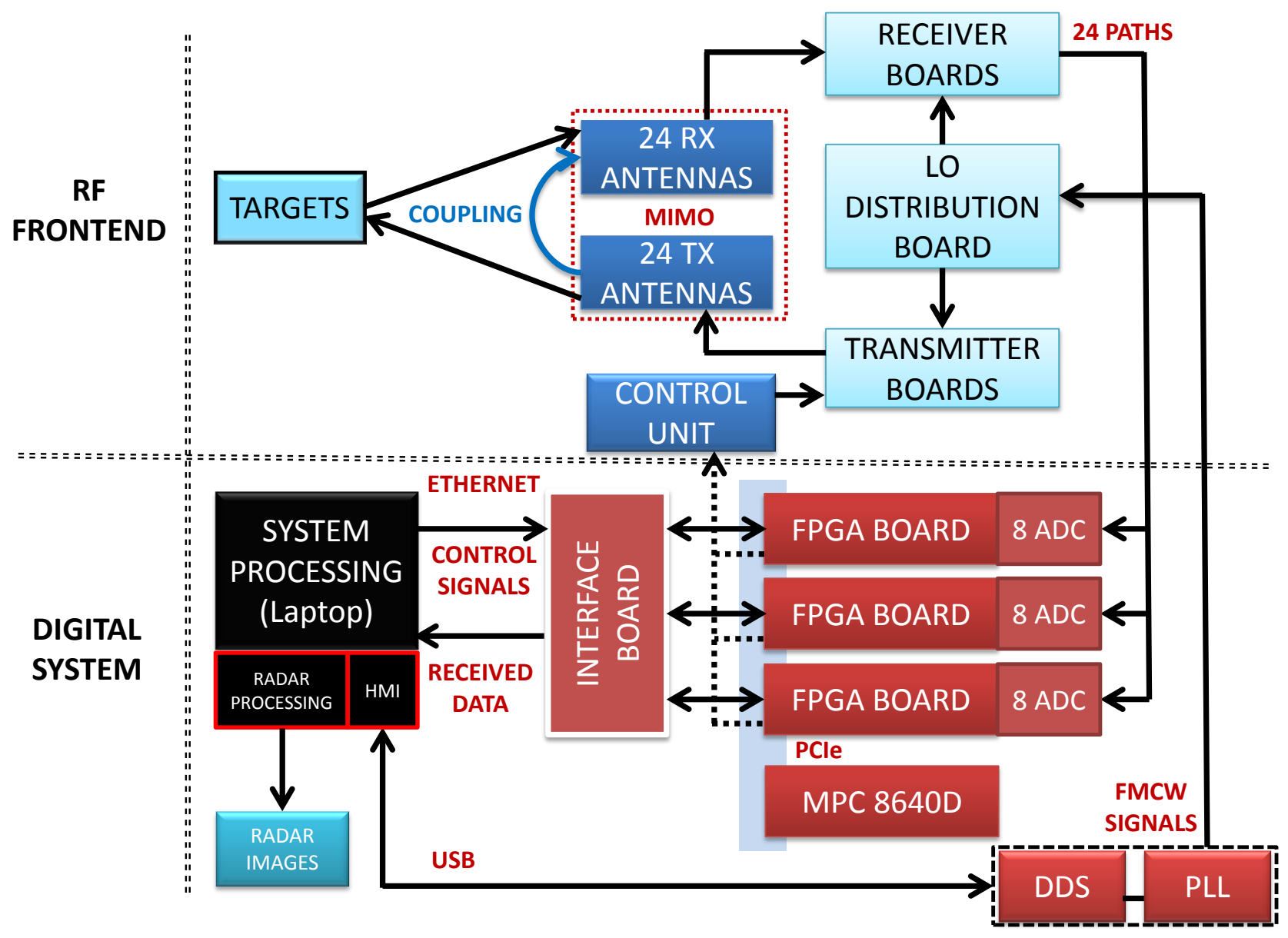

Figure 3.1: System Architecture of the presented MIMO Radar.

urable transmit waveforms and three Field Programmable Gate Array (FPGA) cards with Analog-to-Digital Converter (ADC) units for parallel hardware-based signal acquisition, radar signal processing and radar image generation. A complete description of the digital system is described in [2].

A graphic Human-Machine Interface (HMI) on the system processing workstation sends the signal generation parameters to the DDS and PLL element and the generated FMCW ramp signals are distributed by the LO signal distribution to the transmitter boards and antennas. The control unit selects individual transmit antenna elements in order to apply TDM. The signals reflected from the targets are received through the receiver antennas with multiple receive paths and are processed in parallel in the digital unit. The pre-processed data is then sent back to the offline software based radar signal processing functions on the workstation that apply digital beam-forming techniques to generate the final radar images. 


\subsection{MIMO Array Configuration}

A MIMO radar consist of two sets of multiple transmit (TX) and receive (RX) antennas. In this work, orthogonality between the transmitted signals of the channels is obtained through the use of a TDM architecture of the transmitters. The equivalent virtual array can be calculated as the discrete convolution of the overall RX and TX element positions.

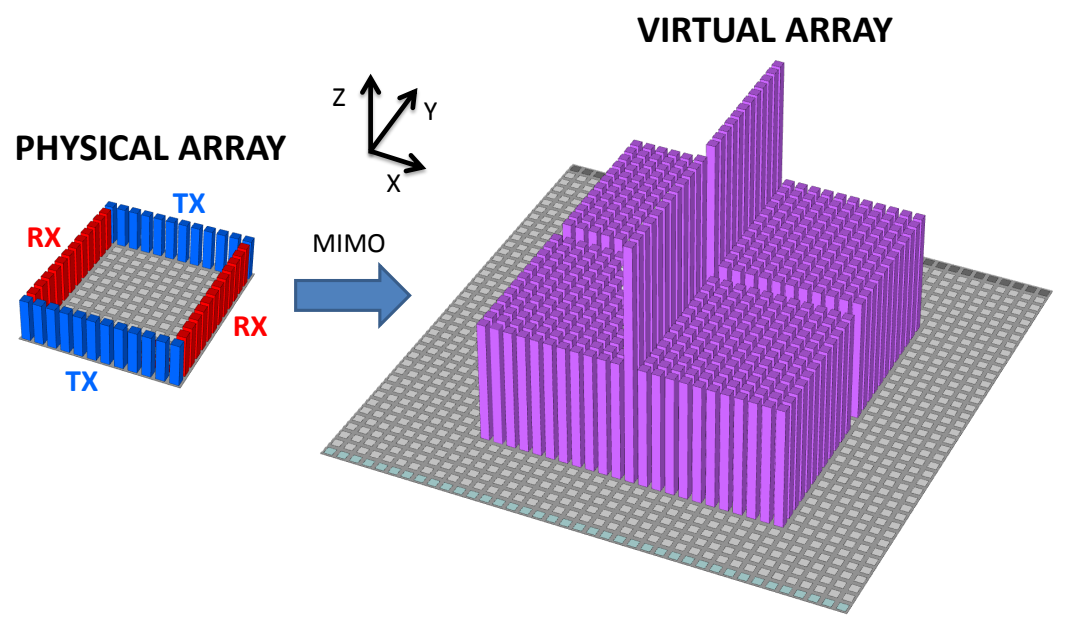

Figure 3.2: Schematic representation of the MIMO antenna configuration with the physical array (left) showing the $24 T X$ antennas in blue, the $24 R X$ antennas in red and the resulting virtual array (right).

In this work, the fabricated antenna elements are based on Tapered Slot Antennas (TSA) [3]. Fig. 3.2 depicts the positions of the antennas' phase centers and the corresponding MIMO virtual array. RX elements (in red) and TX elements (in blue) are placed forming a rectangle. In this manner, the equivalent virtual array has a surface that is four times bigger than the one of a fully populated array with the same perimeter. This leads to an improvement in the resolution of a factor of two in each axis. Thus, the angular resolution is approximately expressed as [4] (uniform array):

$$
\Delta \theta_{3 d B} \approx 50^{\circ} \frac{\lambda_{0}}{d N_{\text {virtual }}} \approx 50^{\circ} \frac{\lambda_{0}}{d 2 N_{\text {populated }}} \approx 2.5^{\circ}
$$

with $\lambda_{0}$ being the wavelength, $N_{\text {virtual }}$ the number of virtual elements in either x- or y-direction, $N_{\text {populated }}$ the number of elements of an equivalent fully populated array with the same perimeter as our physical array and $d$ the distance between elements. The higher 
purple bars in the right of Fig. 3.2 are redundancy elements. Concerning the redundant elements, there are two important aspects that need to be considered. First, the redundant elements are simply discarded in the beam-forming and image generation algorithms of the processing unit and therefore not harming the reconstructed radar image. Second, in some fast-moving scenarios the switching time could be slower than the image changes. As a consequence of the redundancy, the same virtual element is calculated at two different times. This can be exploited in order to evaluate if the radar images are fitting the reality. Defining corresponding algorithms will be one important aspect of our future work. Regarding the empty row which appears in the center of Fig. 3.2, an interpolation of the data is performed, according to what has been described previously in [4]. There are some MIMO array configurations in which the empty row and the redundancy can be avoided, although it is incompatible with the chosen sandwich configuration in which RX and TX panels are stacked (see Fig. 3.25).

In order to reduce the coupling between radiating elements and to increase the angular resolution, the distance between antennas was chosen greater than $\frac{\lambda_{0}}{2}=9.7 \mathrm{~mm}$. As a result, grating lobes appear. Since the desired Field Of View (FOV) is $\pm 25^{\circ}$, the system was optimized placing the elements with $d_{y}=d_{x}=15 \mathrm{~mm}$ of spacing. In such manner, the grating lobes are outside of the desired field of view and an improvement of approximately $10 \mathrm{~dB}$ in the coupling is achieved.
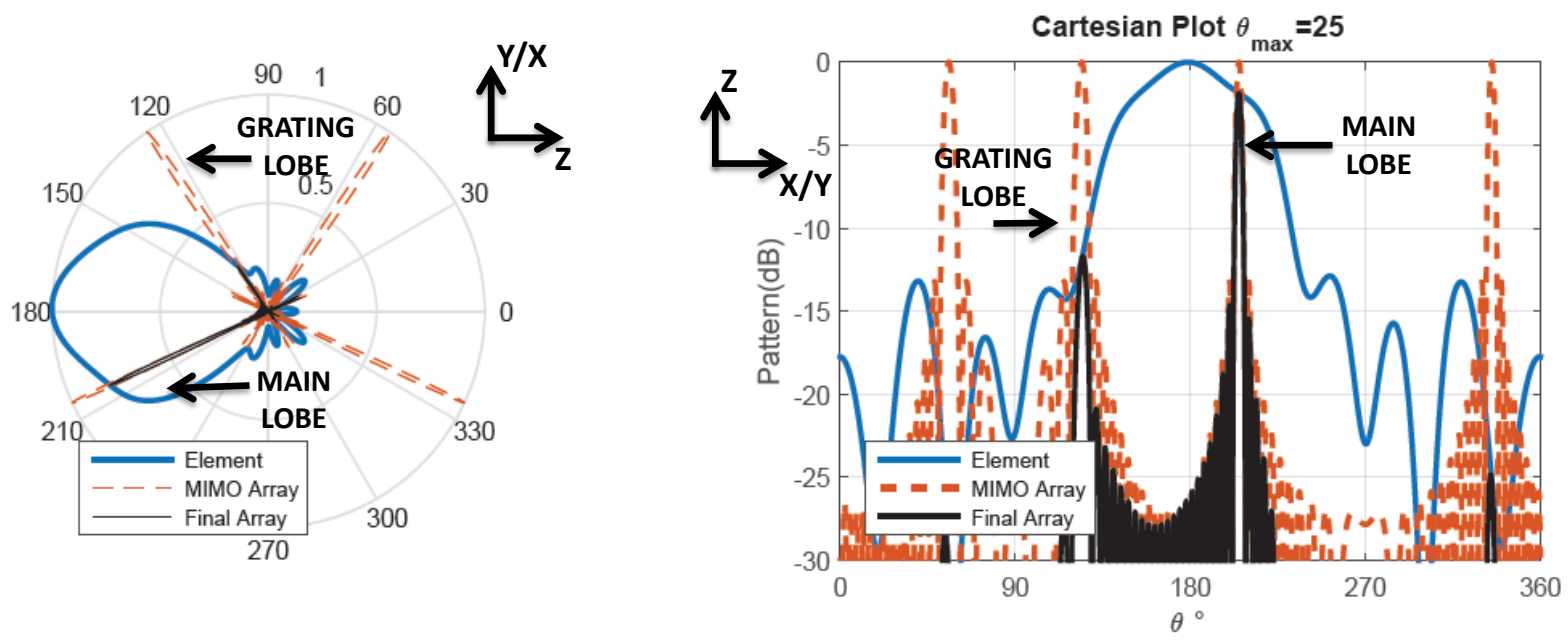

Figure 3.3: The graph illustrates the virtual array pattern with the designed maximum beamsteering of $\theta_{0}=25^{\circ}$ in red, the single element in blue and the resultant multiplication of both in black. The patterns are displayed in polar (left) and cartesian (right) coordinates.

Fig. 3.3 presents a calculation of the resultant antenna pattern. The left picture is dedicated to the antenna pattern of the virtual array in polar coordinates (dashed curve) with the maximum beam steering of $\theta_{0}=25^{\circ}$, single element in blue and the resultant multiplication. As the image shows, the grating lobe remains below $-12 \mathrm{~dB}$ on the desired 
FOV. On the right part of Fig. 3.3 the same curve is plotted in cartesian coordinates. Since the beam-forming is carried out at the digital signal processing side with a delay-and-sum beamformer algorithm, no phase shifters are needed.

\subsection{The RF Frontend}

The focus of this section is on the RF front-end. The hardware was optimized for a frequency range of $15 \mathrm{GHz}$ to $17.5 \mathrm{GHz}$, which is allocated in Germany for non-navigational radio-location services.

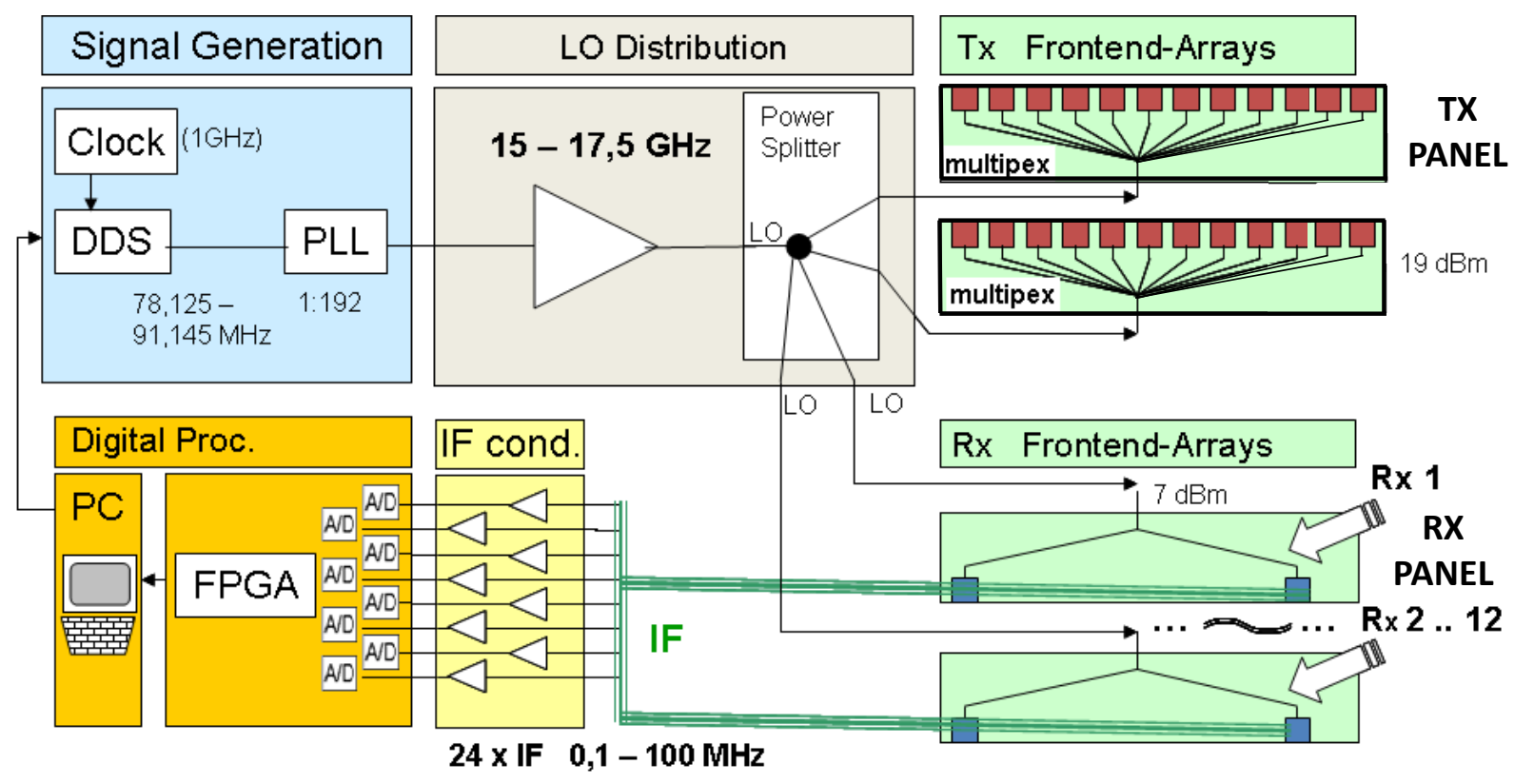

Figure 3.4: Sketch of the RF system.

Fig. 3.4 illustrates the main blocks of the fabricated RF system. The signal generation block feeds the Local oscillator (LO) distribution board with a ramp signal, which must be comprised within 15 and $17.5 \mathrm{GHz}$. The LO distribution board divides this signal into 16 coherent (same amplitude and same phase) signals. Two of the output ports of the LO board are connected with the TX Frontend-Arrays. In them the signal is amplified and guided to only one antenna among the 12 available, in such a manner that the previously mentioned TDM is enabled. The mixers of the 12 RX Frontend-Arrays are also connected to the signal distribution board and to the received signal from the antenna. Consequently, the characteristic IF signal of a FMCW system with the target information is generated and processed in the Digital Processing Unit. The two remaining ports of the LO board are matched with a $50 \Omega$ load in the working configuration of the radar. Furthermore, these ports are used for characterization purposes. 


\subsection{Building Blocks Design}

Until now a top bottom approach has been addressed, accordingly, at first a complete radar design has been performed and afterwards a subsystem design. Once the components are selected, a different strategy is followed. At the beginning the components are characterized, then the subsystems, later the systems and at the end the complete radar is assembled, therefore a bottom top approach is pursued.

\subsubsection{Calibration Kit Design}

First of all, in order to carry out the de-embedding process of the connectors of the Device Under Test (DUT) a Thru, Reflect, Line (TRL) [6] calibration kit is designed. At least three standards are needed for this calibration procedure. The Thru standard must be a line of length zero o greater, but always smaller than the standard Line, the Thru standard is defining the calibration plane. The Reflect standard can be anything with a high reflection. The length of the Reflect standard is typically the same as the Thru standard, although it can be different if the time delay is known. The Line standard is a line of same impedance and propagation constant as the Thru. A TRL calibration kit with broad frequency coverage requires multiple Line standards. The phase difference between the Line and the Thru must be greater than 20 degrees and less than 160 degrees.

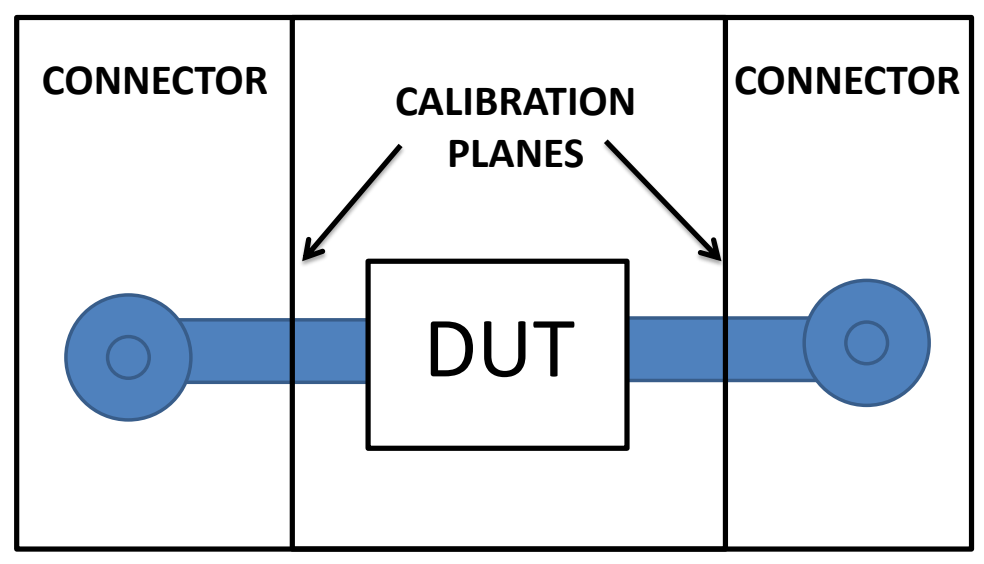

Figure 3.5: Representation of a generic building block.

The layer stack-up of the boards used in this chapter is presented in Fig. 3.6. The RO4003 material is a high frequency suitable material from Rogers corporation with $h=0.203 \mathrm{~mm}, \epsilon_{r}=3.55$ and $\tan \delta=0.0027$. The main function of the Flame Retardant (FR4) $(h=1 \mathrm{~mm})$ is to give physical stability. The FR4 also allows to add extra metal layers for routing of Direct Current (DC) signals. 


\section{$\mathrm{RF} / \mathrm{DC} \quad$ Antennas (TSA)}

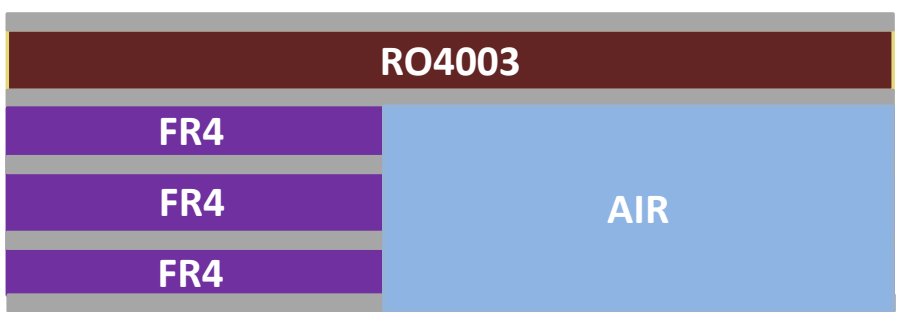

Figure 3.6: Representation of the used layer stack-up. The FR4 layers are milled where the TSA are located in order to enable the proper radiation of the antennas.

A Thru microstrip line standard of $10 \mathrm{~mm}$, which sets a calibration plane of $5 \mathrm{~mm}$ distance of the port together with two microstrip lines terminated in open circuit of $5 \mathrm{~mm}$ are set. Concerning the Lines standards, three different Lines are designed in order to cover the frequency band contained between 1 and $40 \mathrm{GHz}$. The effective permittivity constant $\epsilon_{\text {eff }}$ of the microstrip line is calculated with the TXLine tool included in Advancing the Wireless Revolution (AWR) to be 2.61. Table 3.1 includes a summary of the length delays and frequency of operation of each standard.

\begin{tabular}{cccc} 
Standard & Length & Time delay at central frequency & Frequency \\
\hline Thru & $0.5 \mathrm{~mm}$ & $0 \mathrm{ps}$ & - \\
Open & $0.5 \mathrm{~mm}$ & $0 \mathrm{ps}$ & - \\
Line 1 & $0.5+2 \mathrm{~mm}$ & $10.770 \mathrm{ps}$ & $6-40 \mathrm{GHz}$ \\
Line 2 & $0.5+6 \mathrm{~mm}$ & $32.311 \mathrm{ps}$ & $2-12 \mathrm{GHz}$ \\
Line 1 & $0.5+12 \mathrm{~mm}$ & $64.622 \mathrm{ps}$ & $1-6 \mathrm{GHz}$
\end{tabular}

Table 3.1: Description of main parameters of the calibration kit

Fig. 3.7 depicts the fabricated calibration kit with two different connectors. Three 50 $\Omega$ lines with length of $25 \mathrm{~mm}, 50 \mathrm{~mm}$ amd $100 \mathrm{~mm}$ are also included in order to verify the measurement and to generate an accurate microstrip line model. The width of these lines is $w_{50}=0.422 \mathrm{~mm}$. The surface plating used is the Electroless Nickel Immesion Gold (ENIG). It is based on an electroless nickel plating covered with a thin layer of immersion gold, which protects the nickel from oxidation. As advantages ENIG produces a flat surface and a long shelf life for a lower price than hard gold. The main disadvantage is that for RF signals nickel, which is a ferromagnetic material, produces losses.

It must be noted that a two port E8363B Agilent NA is used in all measurements of this work. 


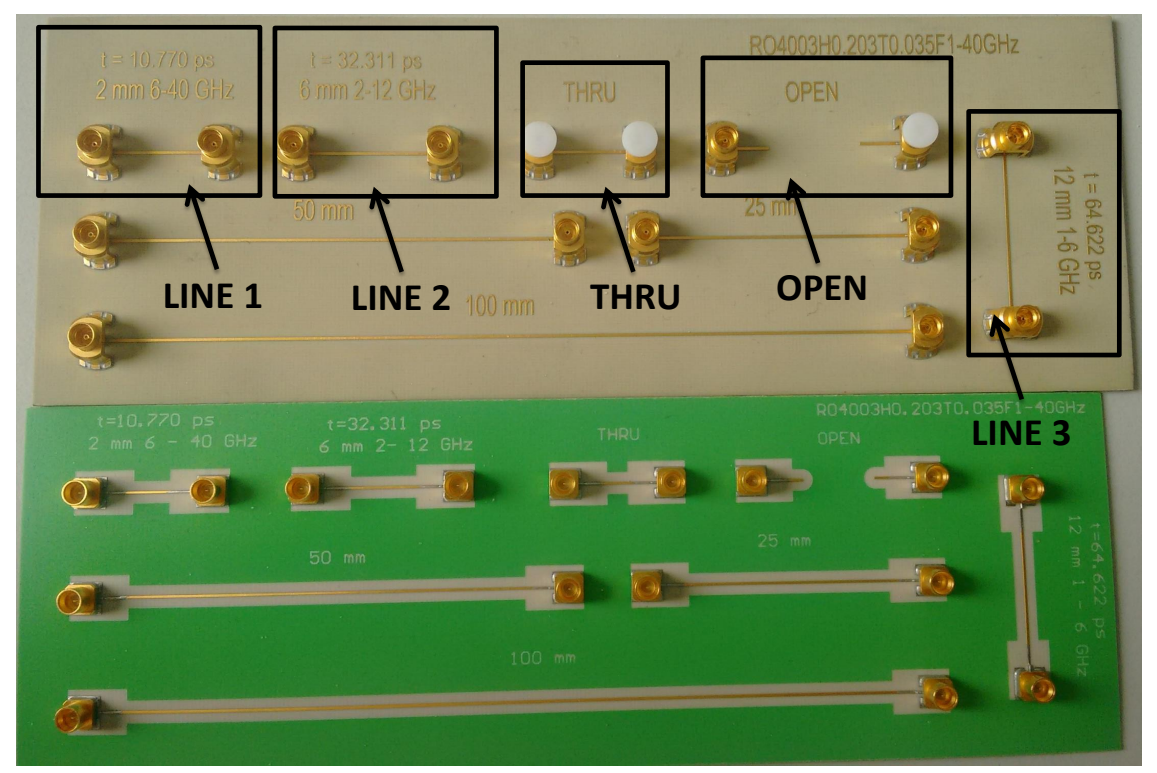

Figure 3.7: Fotography of the Calibration kit with different connectors.

\begin{tabular}{l|ll|} 
Name & Value & Description \\
\hline R Er & 3.67 & Relative dielectric constant \\
\hline $\mathbf{R}$ H & 0.203 & Substrate thickness \\
\hline R T & 0.035 & Conductor thickness \\
\hline R Rho & 6 & Metal bulk resistivity normalized to gold \\
\hline R Tand & 0.0065 & Loss tangent of dielectric \\
\hline R ErNom & 3.55 & Nominal relative dielectric constant, used for X-models \\
W Name & RO4003 & Substrate name
\end{tabular}

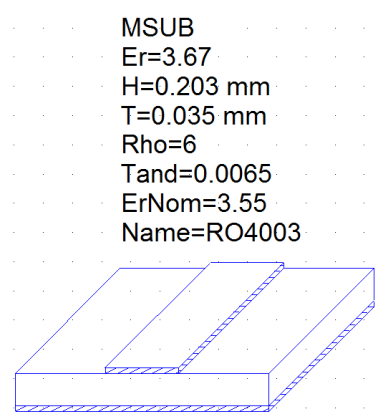

Figure 3.8: Model of microstrip line in AWR.

\subsubsection{Microstrip Line Model}

In order to characterize a subsystem, the following procedure is followed. First, a measurement of the S-parameters of the included RF components is performed. After that, the measured S-parameters are linked with the microstrip line model. In order to calculate this model, the main parameters of the microstrip line are selected from the correspondent datasheet, the dielectric constant is $\epsilon_{r}=3.55$, the dissipation factor is $\tan (\delta)=0.0027$, the substrate thickness is $\mathrm{h}=0.203 \mathrm{~mm}$, metal thickness strip is $\mathrm{t}=0.035 \mathrm{~mm}$ and bulk resistivity of nickel at $20 \mathrm{C}$ is $\rho=6.9 \mu \Omega \mathrm{cm}$. In order to fine tune these parameters, the measurement of 3 microstrip lines with lengths of $100 \mathrm{~mm}, 50 \mathrm{~mm}$ and $25 \mathrm{~mm}$ are used.

Fig. 3.8 shows the AWR model of a microstrip line with final value of the parameters and their description: $\epsilon_{r}=3.67$ instead $3.55, \tan (\delta)=0.0065$ instead 0.0027 and $\rho=6 \mu \Omega \mathrm{cm}$ 
instead $6.9 \mu \Omega \mathrm{cm}$. The substrate thickness (h) and conductor thickness (t) remain constant.

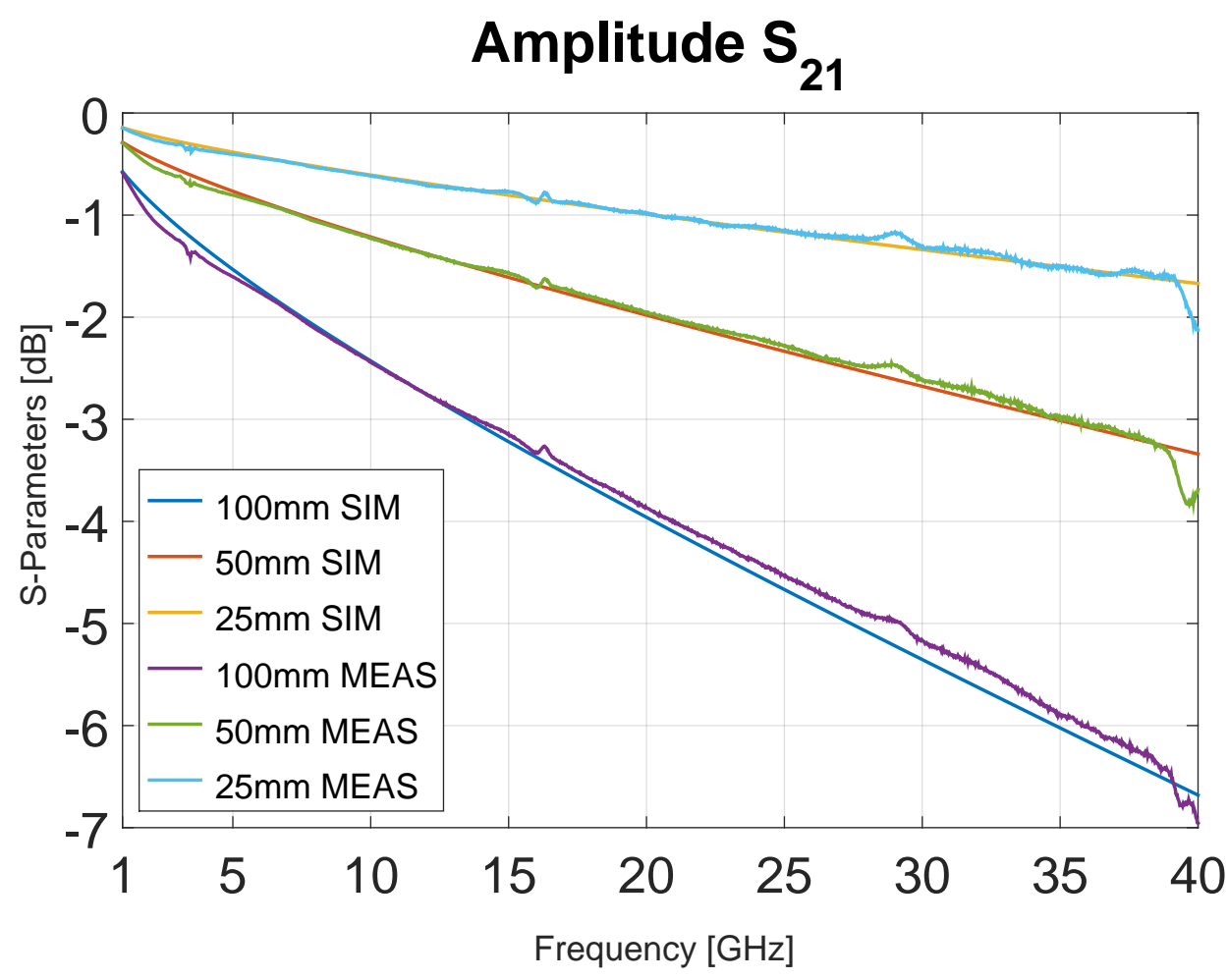

Figure 3.9: Comparison of the $S_{21}$ of between three $50 \Omega$ lines of different length (25 $\mathrm{mm}, 50 \mathrm{~mm}$ and $100 \mathrm{~mm}$ ) and its respective $S_{21}$ simulation produced with the presented model.

Fig. 3.9 illustrates a comparison between the measurement of the $S_{21}$ of three $50 \Omega$ lines with different lengths, $100 \mathrm{~mm}, 50 \mathrm{~mm}$ and $25 \mathrm{~mm}$, repectively with the simulated $S_{21}$ of three $50 \Omega$ lines with the previously described model. These measurements do not include the effect of the connectors. The model fits rather well the amplitude simulation over the whole frequency range.

Fig. 3.10 depicts the comparison of the phase of the $S_{21}$ of three different lines (with length of $100 \mathrm{~mm} 50 \mathrm{~mm}$ and $25 \mathrm{~mm}$ ) and their simulation with the presented model. The curves in blue represent the simulated model while the red curves represent the simulation. The red curves fit well the simulation, indeed they overlap the blue curves almost over the whole frequency range. The measured attenuation of the microstrip line is $\alpha \approx 0.3 \mathrm{~dB} / \mathrm{cm}$.

Now that a microstrip line model is defined, in the following subsections the measured S-Parameter of the building blocks (switches, amplifiers and power splitters) are presented. These measurements are incorporated in a system simulation together with the presented microstrip line model. 

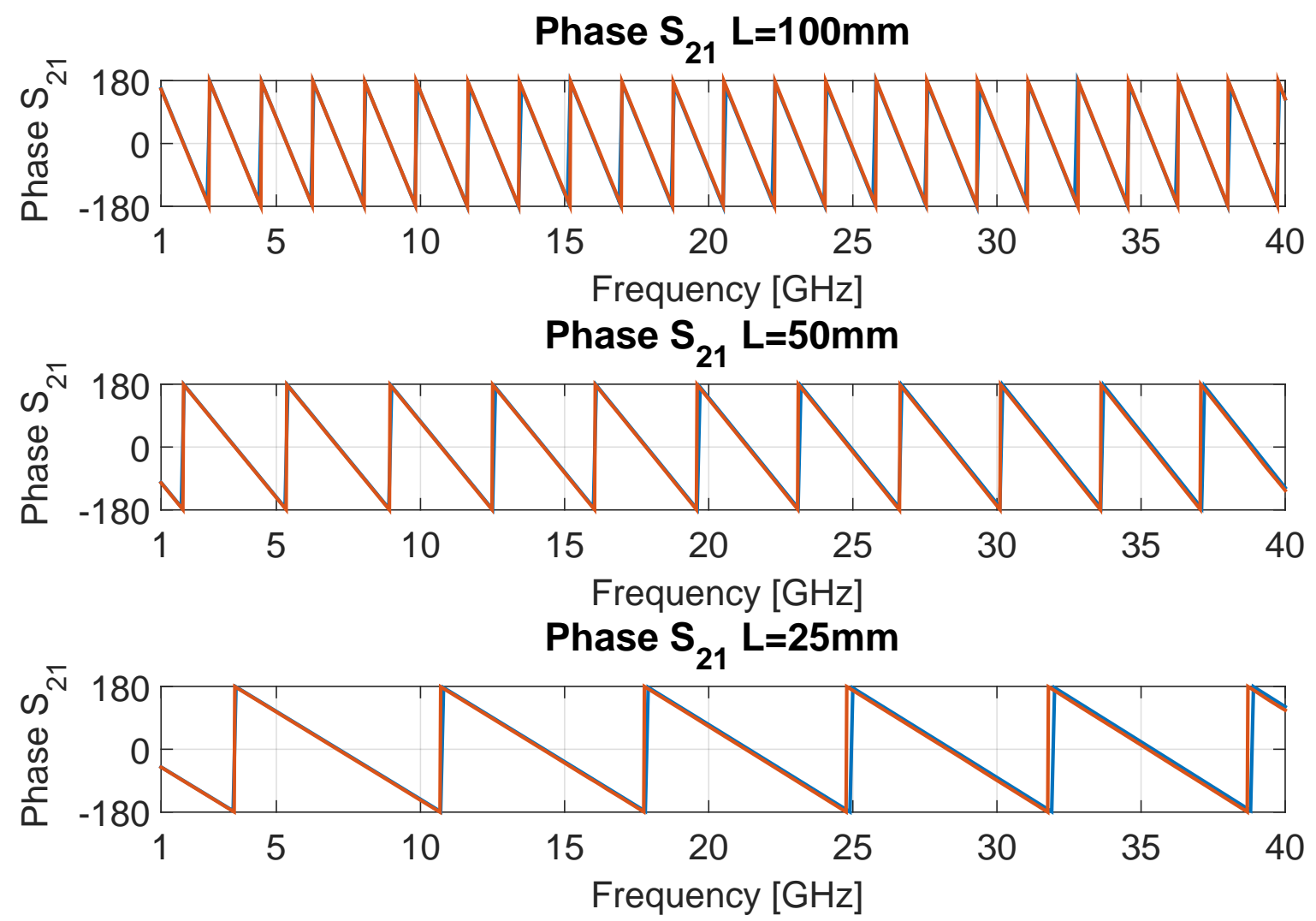

Figure 3.10: Phase comparison of the phase of the $S_{21}$ of three different lines (with length of $100 \mathrm{~mm} 50 \mathrm{~mm}$ and $25 \mathrm{~mm}$ ) and their simulation with the presented model

\subsubsection{Amplifiers}

The RF signals' amplitude flowing through the microstrip line decreases due to radiation, dielectric and conductive losses [7]. Furthermore, other required components, such as switches, filters and mixers also produce an increment of the insertion loss. Moreover, the signal strength of an electromagnetic wave in free space has an inversely proportional dependency with the distance [8]. As a consequence, a radar sensor needs a strong RF signal in order to achieve a reasonable maximum range (depending on the application). One way to approach this problem is adding amplifiers to the circuit. In this subsection, two different amplifiers off-the-shelf, which are used as building blocks for the fronted, are presented (HMC451LC3 and AVA-24+). These amplifiers are used as a building block in 
more front-ends.

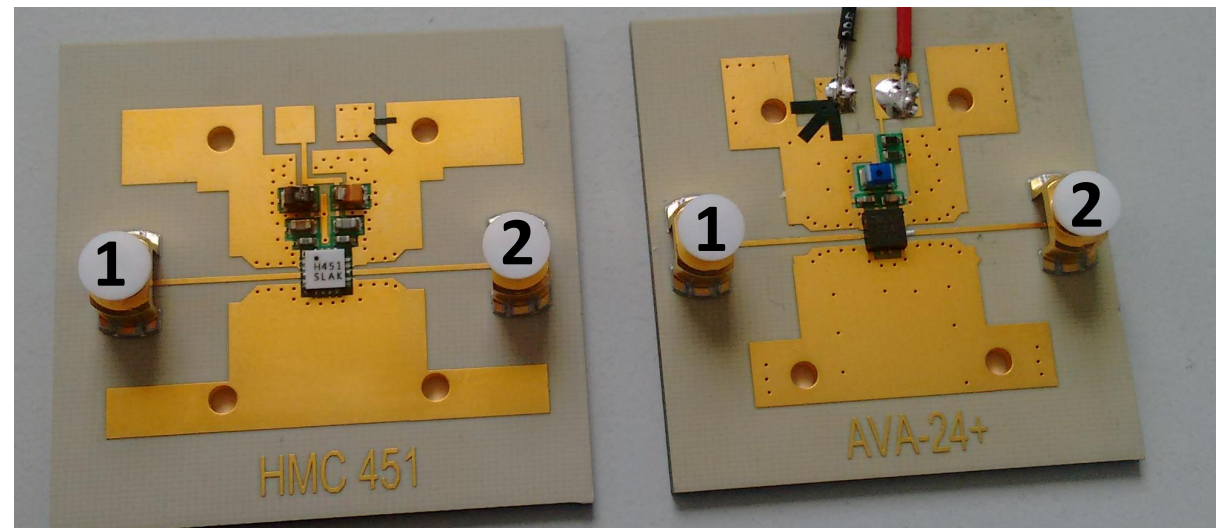

Figure 3.11: Photography of the measured test amplifiers, HMC451 (left) and AVA-24+ (right).

Fig. 3.11 introduces a photography of the measured test amplifiers. The off-the shelf IC are integrated in a simple PCB with a transition from coplanar grounded line to microstrip line. Fig. 3.12 illustrates the measured S-parameters of the HMC 451 (left) and the AVA$24+$ (right).
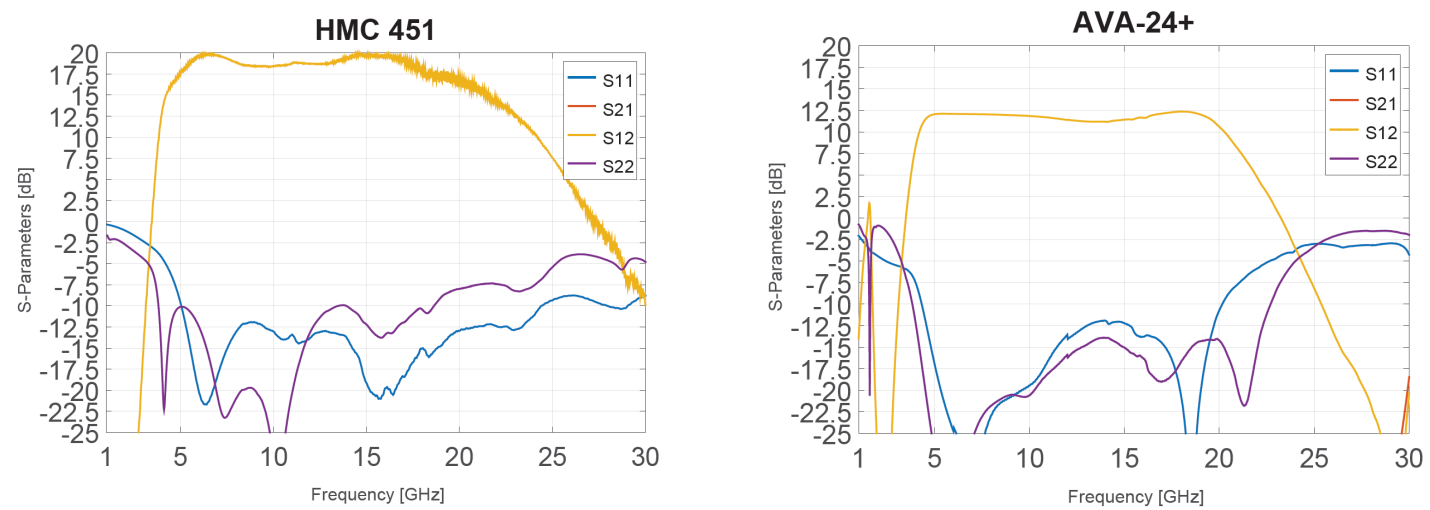

Figure 3.12: Measured S-parameters of the test amplifiers, HMC451 (left) and AVA-24+ (right).

The measured results of the HMC 451 and AVA-24+ S-parameters are in agreement with the specifications of the datasheets with gains of $20 \mathrm{~dB}$ and $12 \mathrm{~dB}$, respectively. The frequency band of operation is from 5 to $20 \mathrm{GHz}$, approximately. However, the targeted frequency range for this front-end is from $15 \mathrm{GHz}$ to $17.5 \mathrm{GHz}$. The isolation is very small in both cases and therefore not visible in the plots. Input and output return loss are below $10 \mathrm{~dB}$ in the frequency range of interest. This measurement proves that the selected amplifiers are suitable for the radar system. 


\subsubsection{Power Divider}

For many RF-systems, as for instance a radar, power dividers are key components that are used to combine or split a signal. On the one hand, classical T-junctions accomplish this task in a simple way, on the other hand, they have poor isolation, a fact that makes Tjunctions less popular in modern microwave circuits. Although E. J. Wilkinson presented a new power divider [7] solving the isolation problem, a traditional Wilkinson divider uses a $\frac{\lambda}{4}$ impedance and a $\sqrt{2} Z_{0}$ resistor, when $Z_{0}$ is the reference characteristic impedance of the circuit. A deeper analysis for this circuit and a broadband version are presented in chapter 5 .

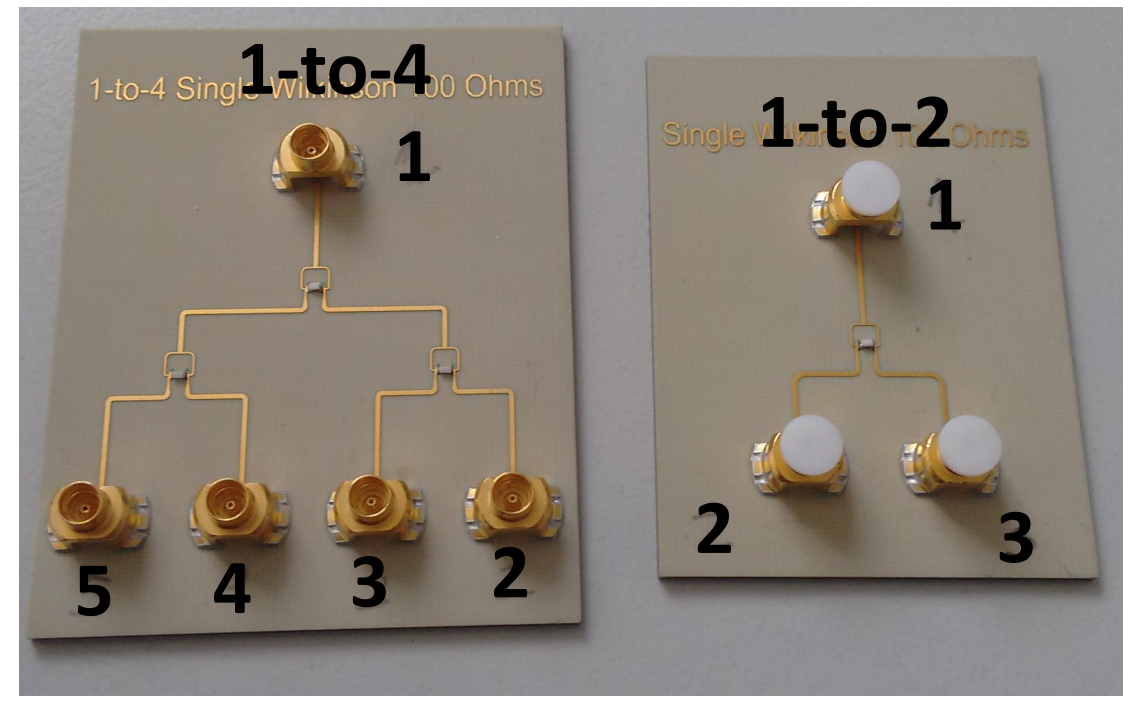

Figure 3.13: Photography of the building blocks. A 1-to4 Wilkinson divider (left) and 1-to-2 Wilkinson (right).

Fig. 3.13 shows a photography of the fabricated Wilkinson power dividers in the previously described substrate (see section 3.6). A microstrip line with characteristic impedance of $Z_{c}=50 \Omega$ and a width of $w_{50}=0.422 \mathrm{~mm}$, is used as a reference in all ports. After the bifurcation, two parallel microstrip lines with a characteristic impedance of $Z_{c}^{\prime}=\sqrt{2} Z_{c}=\sqrt{2} \cdot 50=70.71 \Omega$ and thus, width of $w_{70.71}=0.177 \mathrm{~mm}$ and length of $\frac{\lambda}{4}=2.95 \mathrm{~mm}$ are placed before the $2 Z_{c}=100 \Omega$ resistor. In the 1-to-2 Wilkinson divisor version, the described structured is followed by the out ports. In contrast, the 1-to-4 Wilkinson divisor version is a simple concatenation of Wilkision divisors. Theoretically, a Wilkinson divisor can also be designed in a single stage. However, there are two difficulties. The first one is that the isolation between ports 4 and 2 would worsen, since they cannot be connected with a resistor due to geometrical reasons (analog to port 2 and 3 in Fig. 4.22). The second one is that the minimum track width that our supplier can fabricate is $w_{\min }=0.1 \mathrm{~mm}$. A direct 1-to-4 power divisor needs four arms in parallel with an impedance of $Z_{c}^{\prime}=\sqrt{4} Z_{c}=100 \Omega$ which is exactly $w_{100}=0.1 \mathrm{~mm}$. In this case, a more conservative approach was selected, however, in chapter 4 a 1 to 3 power divisor 
which overcomes the difficulty number 1 and in the chapter 5 a very broadband Wilkinson divisor which overcomes the difficulty 2 are measured with good results.
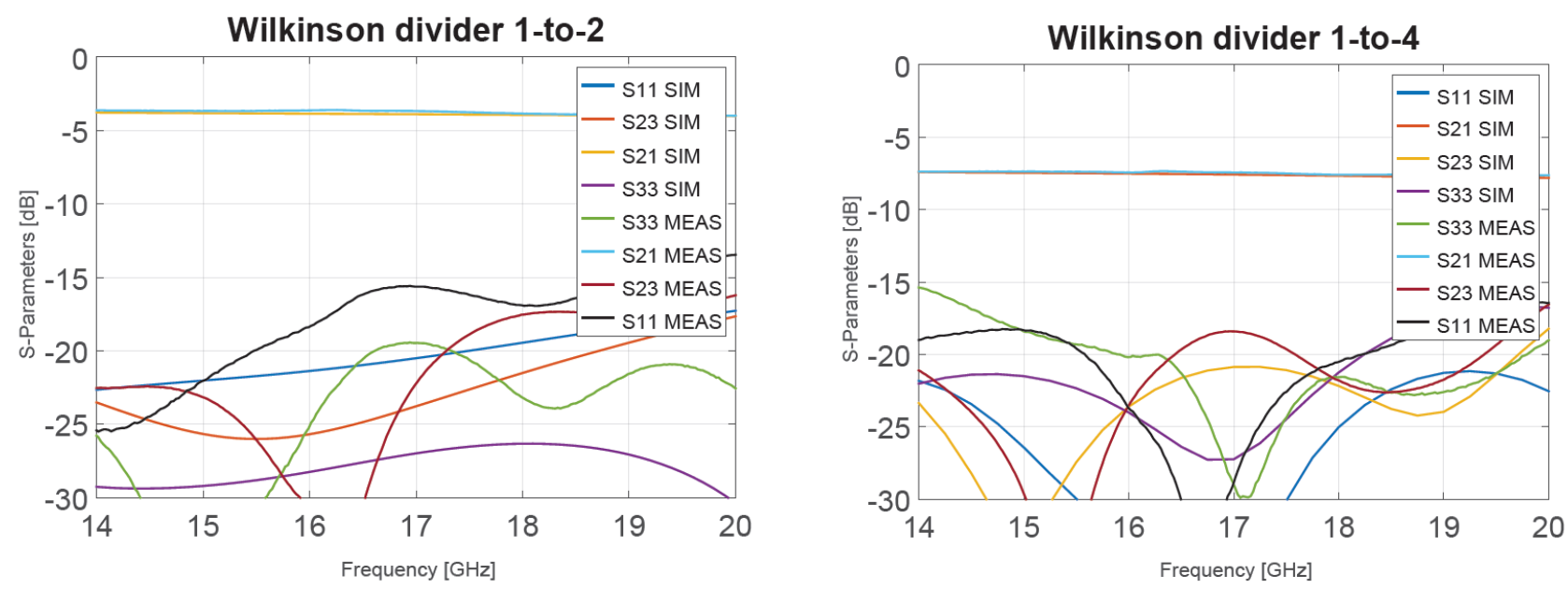

Figure 3.14: Measuremed S-parameters of 1-to-2 Wilkinson power divisor (left) adn 1-to4 power divisor (right).

Fig. 3.14 illustrates the measured S-parameters of the presented devices. For clarity reasons, only the simulated and measured insertion loss $\left(S_{21}\right)$, input return loss $\left(S_{11}\right)$, isolation $\left(S_{23}\right)$ and output return loss $\left(S_{33}\right)$ of both structures are depicted. The simulated S-parameters are designed with an equation based model of AWR. The 1-to-2 Wilkinson presents a $S_{21}$ of approximately $-3.8 \mathrm{~dB}$. This result fulfills the specifications over the targeted frequency range. It must be noted, that a half of the power is conducted from port 1 to port 2 (this is approximately $-3 \mathrm{~dB}$ ) and that the $S_{21}$ also includes the losses in the microstrip line of length $L_{1}=2.5 \mathrm{~cm}\left(L_{1} \alpha \approx 0.75 \mathrm{~dB}\right)$. The isolation $\left(S_{23}\right)$, input $\left(S_{11}\right)$ and output $\left(S_{33}\right)$ reflections are under $-15 \mathrm{~dB}$ over the targeted frequency range. The 1-to-4 Wilkinson divisor presents a $S_{21}$ of $-7.6 \mathrm{~dB}$. This result fulfills the specifications over the targeted frequency range. It must be noted, that a quarter of the power is conducted from port 1 to port 2 (this is approximately $-6 \mathrm{~dB}$ ) and that the $S_{21}$ also includes the losses in the microstrip line of length $L_{2}=5 \mathrm{~cm}\left(L_{2} \alpha \approx 1.5 \mathrm{~dB}\right)$. The isolation $\left(S_{23}\right)$, input $\left(S_{11}\right)$ and output $\left(S_{33}\right)$ reflections are under -15 dB over the targeted frequency range.

In general terms, the measurements fit rather well the simulation. Some relatively small disparities are due to fabrication tolerances of the PCB and especially on the fact that the simulated resistors are ideal. The measurement results have shown that both fabricated structures can be used as building block for a subsystem design of the radar. The presented structures are the key structures for the LO distribution board, which distribute the ramp signal among the receivers and the transmitters. 


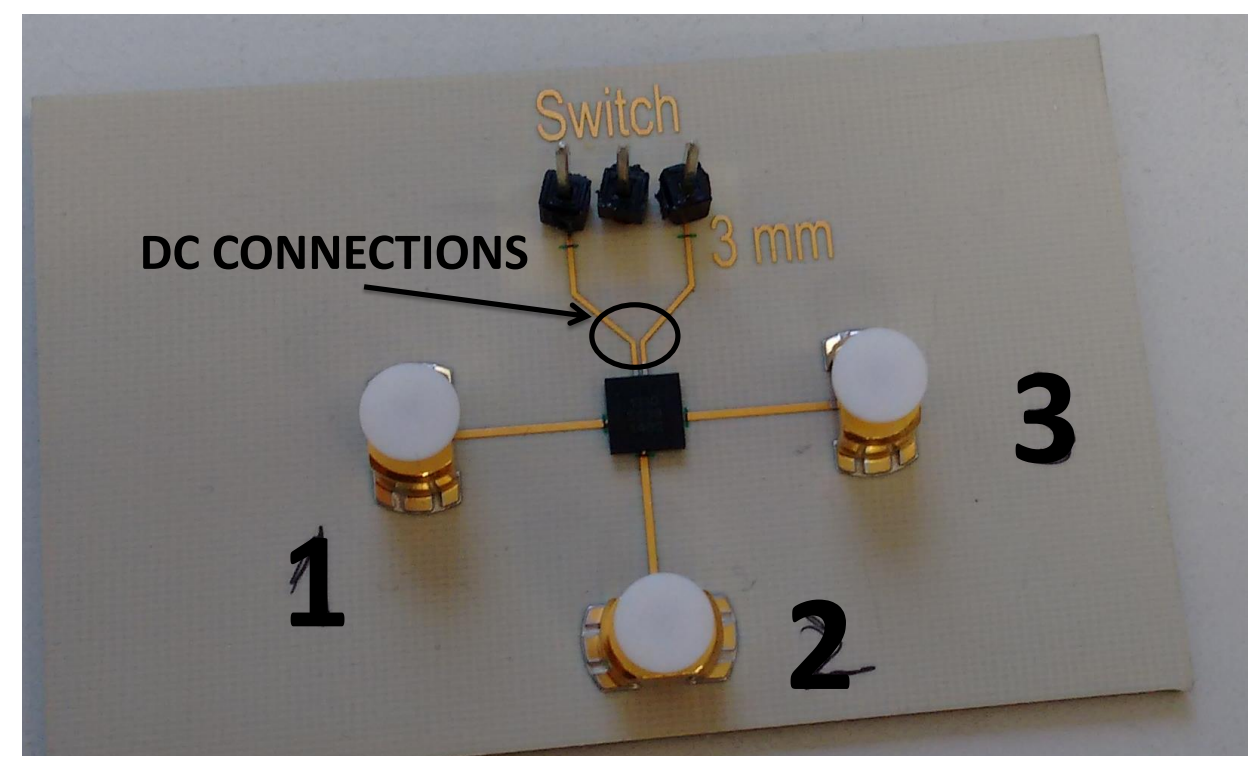

Figure 3.15: Photography of the selected switch connected to microstrip lines.

\subsubsection{RF Switch}

An RF switch is a component that routes an RF signal through different transmission lines (typically grounded coplanar or microstrip line) [10]. RF switches are widely used in RF systems for signal routing, for instance to use an antenna for transmission or reception in a radar system, for adding redundancy paths in a satellite system or to select a calibration standard from a calibration kit. Switches are very important for our system, now that it is based on a TDM paradigm, in which the same signal is sent through different antennas in different times. A digital signal sets the right voltages in the DC ports of the switches in such a manner that the right path is activated and the corresponding antenna sends the RF signal. Some parameters, as the switching time (mostly if the switching time is longer than the time that is needed to to transfer the data to the FPGA), insertion loss or frequency band of operation are very important for the final performance of a MIMO radar. In addition, the switch is also used to generate test ports in our system, in which the signal can be measured in an intermediate point.

Fig. 3.15 shows the fabricated building block of a MASW-008322 GaAs SPDT Switch from MACOM. It is a versatile, broadband, SPDT switch in a lead-free $4 \mathrm{~mm}$ 24-lead PQFN surface plastic package. The design achieves a broadband performance up to 20 GHz. Furthermore, the device achieves a very fast switching time (below $25 \mathrm{~ns}$ ). Port 2 is the import port, which routes the RF signal to the ports 1 or 3 depending on the voltages on the DC connections.

Fig. 3.16 presents the measurement of switch. The measurements agree with the specifications in the data-sheet. Only some of the S-parameters are included in the plot 
due to clarity reasons. The insertion loss (S21) is about $2 \mathrm{~dB}$, the input (S22) and output (S11) reflection are below $15 \mathrm{~dB}$, over the frequency band of operation (from $15 \mathrm{GHz}$ to $17.5 \mathrm{GHz})$. The isolation ( $\mathrm{S} 23)$ is below $-35 \mathrm{~dB}$ over the measured frequency range. The selected switch is in a reflective configuration, therefore the isolated port has full reflection (see S33).

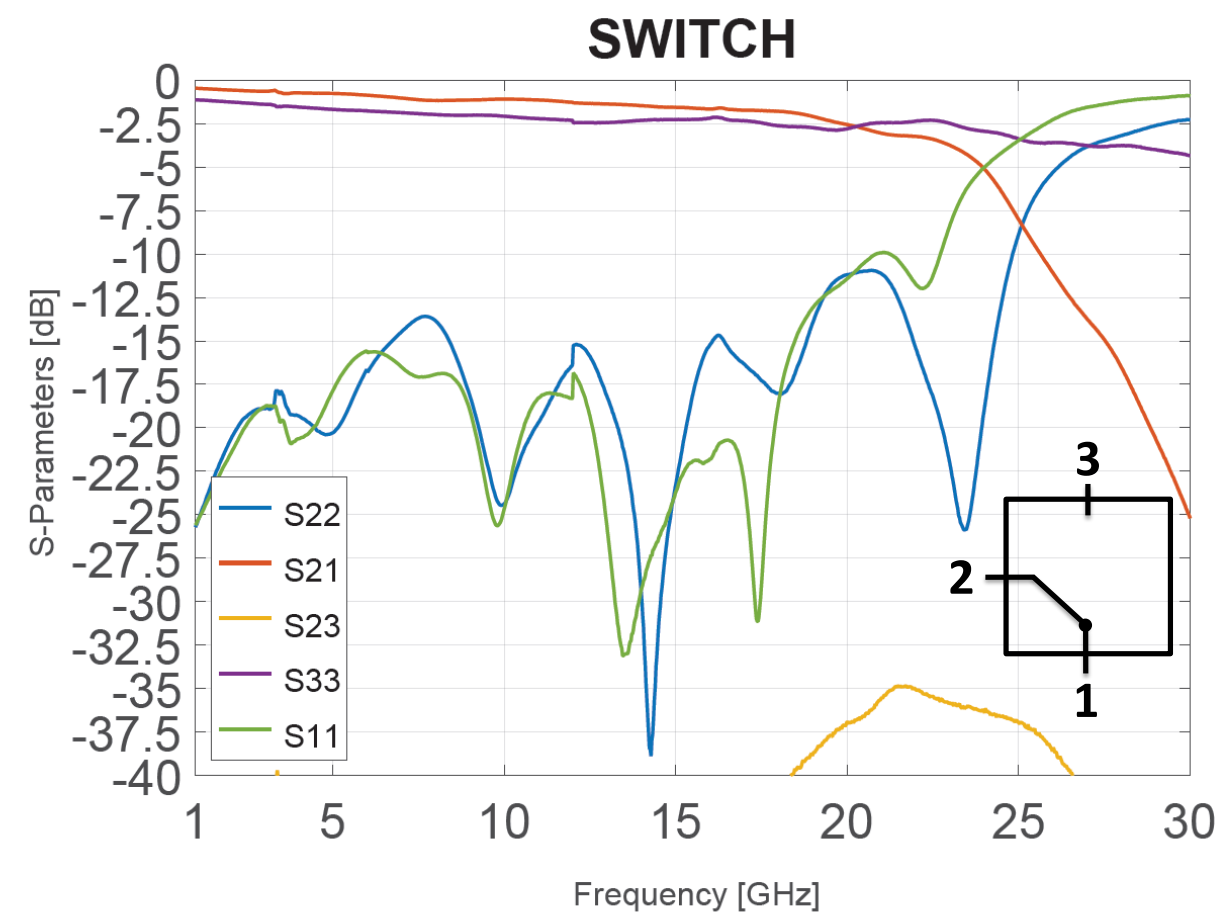

Figure 3.16: Measured S-parameter of the built MASW-008322 GaAs SPDT switch.

In this section, the main building blocks and its measurements of the radar system have been introduced. These structures provide the backbone of the radar presented in this chapter and are a very important part of other radar systems in developed AGI. The gathered information has been used to create a power level based system design and frontend level simulations, which are essential for the correct front-end design.

\subsection{LO Distribution Board}

The LO distribution board feeds the RX and TX boards with the RF signals that are used for transmission and de-ramp at the receiver, where the mixing occurs between the received chirp signals at the RX boards and the transmitted chirp coming from the LO board. The LO distribution board provides a phase-coherent signal from a single input port to 16 output ports. 12 ports feed the 12 RX boards, 2 ports feed the 2 TX boards and 2 ports are designed for testing purposes (these testing ports are matched with loads 
better than $-30 \mathrm{~dB}$ during radar operation time). The structure of the designed boards consists of 4 stages of single resistor Wilkinson power divisors and two stages of commercial amplifier (AVA-24+).

\subsubsection{LO Distribution Board: System Level Considerations}

Firstly, an Excel sheet with the link budget [1] is completed as Fig. 3.17 (right-above) illustrates. In it, the sum of the produced signal gain by the amplifiers likewise the subtraction of losses produced by the connectors, power dividers and microstrip lines is calculated together in order to estimate the overall signal level at each stage of the chain. Another important parameter often described in the power budget is the noise figure, which represents the degradation of the signal-to-noise ratio in the RF chain [12]. The noise figure is calculated in each stage following equation $(3.2)$ :

$$
N F=N F_{1}+\frac{N F_{2}-1}{G_{1}}+\frac{N F_{3}-1}{G_{1} G_{2}} \ldots+\frac{N F_{N}-1}{G_{1} G_{2} \ldots G_{N-1}}
$$

The order of the RF components in the chain is very important since, as equation (3.2) shows, different orders lead to different noise factors. It is well known that specially in receivers (where the input signal has a very small amplitude) a Low Noise Amplifier (LNA), which has a small noise factor and high gain, is very convenient. Therefore, the first component placed in our circuit is an amplifier. Moreover, a row with the $1 \mathrm{~dB}$ Output Compression Point OCP1 is also included. If the Direct Current (DC) power provided to the amplifier is not enough to amplify the signal, the amplifier distorts the signal generating harmonics, which can lead to some electromagnetic compatibility problems, if they are not properly filtered. In addition, if after OCP1 is reached, more input power is applied, the Third-Order Intercept (TOI) point can be reached [13. This is a problem, specially in a communication system in which two different carriers can generate harmonics within the operational bandwidth [14. The next row describes the maximum input power that the components withstand. $P_{\text {in }}, P_{\text {out }}$, Overall Gain and NF-Contr indicate the corresponding calculated values in each stage of the system. The simulation schematic is presented in Fig. 3.17 (left-below). It is based on the measured building blocks and the calculated microstrip line model.

\subsubsection{LO Distribution Board: Simulation and Measurement}

With the accumulated information, a LO distribution board layout was completed in Altium designer. It includes the DC circuits in inner layers and DC connectors on top of the PCB as well as the required RF circuitry. The used layer stack-up is depicted in Fig. 3.6. In order to minimize the occupied surface, an end-fire connector is placed at the input (see Fig. 3.18 A). However, regular SubMiniature version A (SMA) connectors are placed at the 


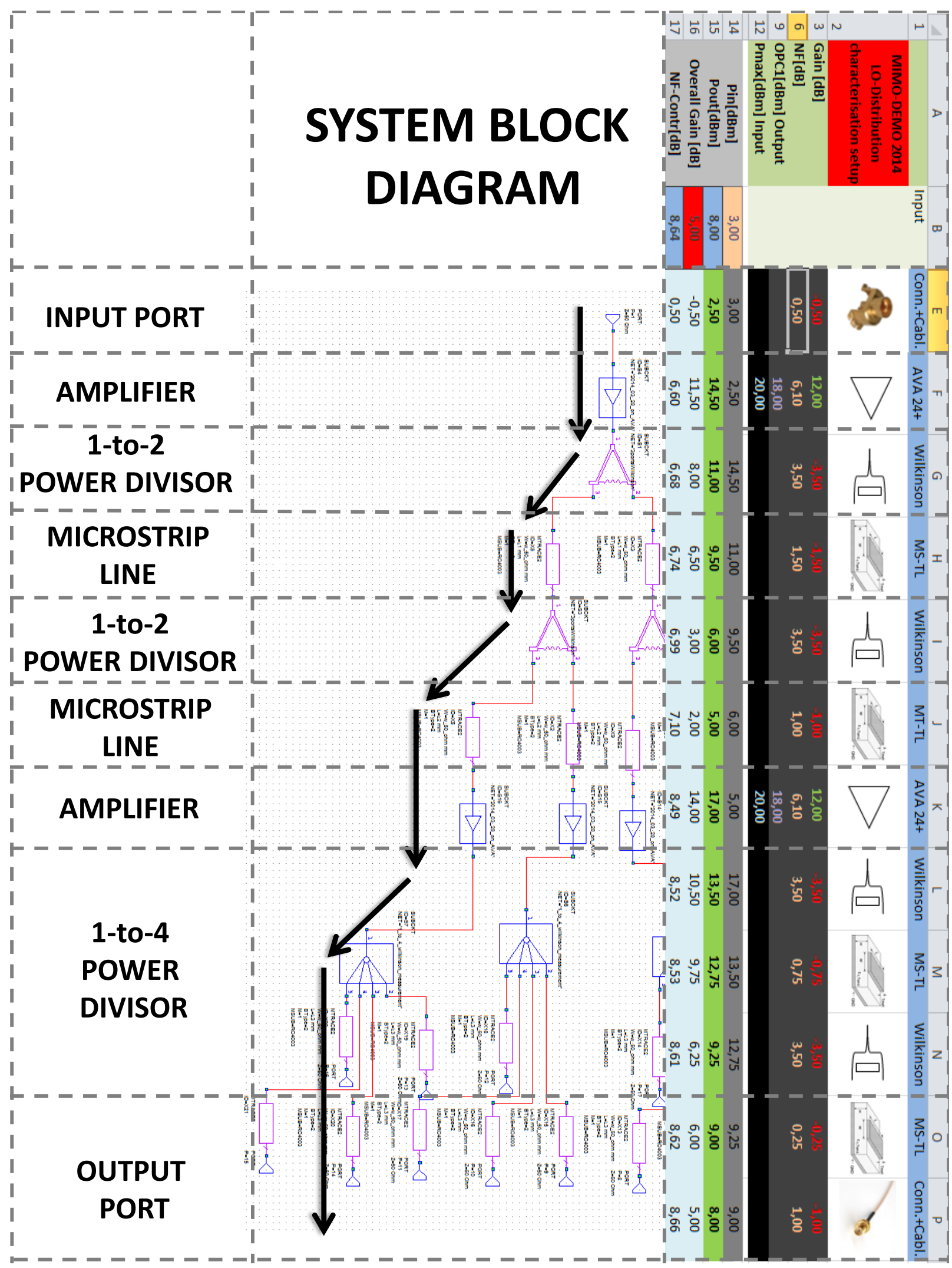

Figure 3.17: System block diagram used for simulation purposes (left-below) and estimated system power levels (right-above). 
outputs. After the fabrication, the components are soldered on top and the S-parameters of the PCB are measured (see Fig. 3.18 B and C).

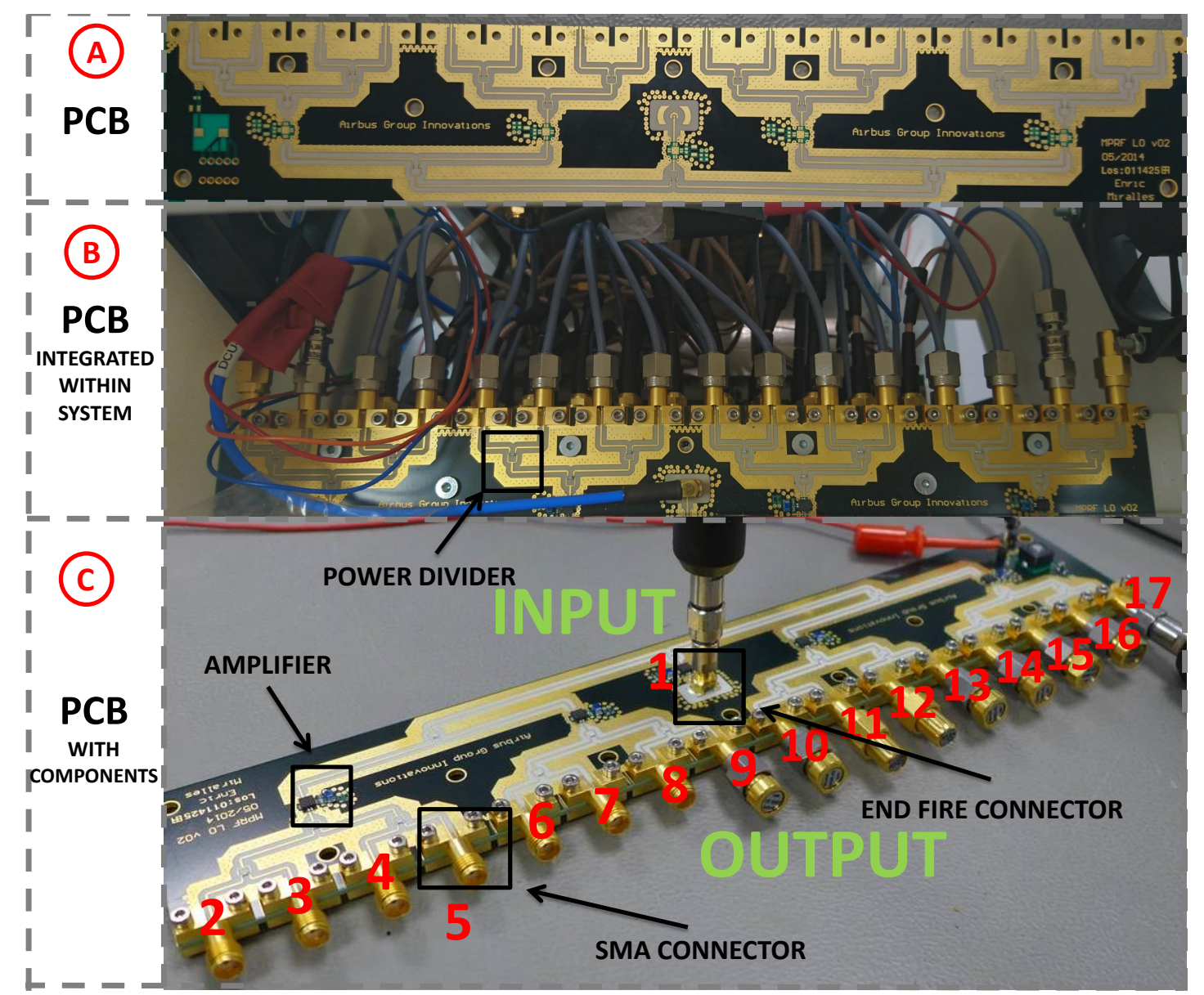

Figure 3.18: Photography of the fabricated LO distribution board. A. PCB without components. B. PCB integrated in the radar system. C. PCB with soldered components.

Fig. 3.19 presents a comparison between simulated and measured S.parameters of the LO distribution board. Considering that the input and outputs ports have different connectors and that the PCB is built with these connectors in the system, a K-connector Short-Open-Load-Thru (SOLT) [15] calibration is performed with a commercial calibration kit. The measurement includes the effect of a transition from the end-fire mini coaxial connector to K-connector. In addition, SMA connectors are mechanically connectible with $\mathrm{K}$ connectors. The measured results are in agreement with the simulation and the calculated values from the system block diagram, especially considering that the input and output connectors are not included in the simulation. The calculation introduced in the system block diagram 3.17 is based on simple equations. Even if it is only a first approximation, it is very helpful to provide a fast and relatively accurate approximation. The 
calculated overall gain in the block diagram is $5 \mathrm{~dB}$, the simulated $S_{21}=6.4 \mathrm{~dB}$ at $17 \mathrm{GHz}$, while the measured $S_{21}=4.2 \mathrm{~dB}$ at $17 \mathrm{GHz}$. The differences between the simulation and the measurement are caused by the lack of the effect of the connectors on the simulation. Regarding the input return loss, the end fire connector and its transition to $\mathrm{K}$ connector placed on the input have a very high performance. For this reason, the measured and simulated input return loss $S_{11}$ match very well. In contrast, the output connectors are very inexpensive SMAs, consequently they affect the output reflection and explain the difference between measured and simulated output return loss $S_{22}$.

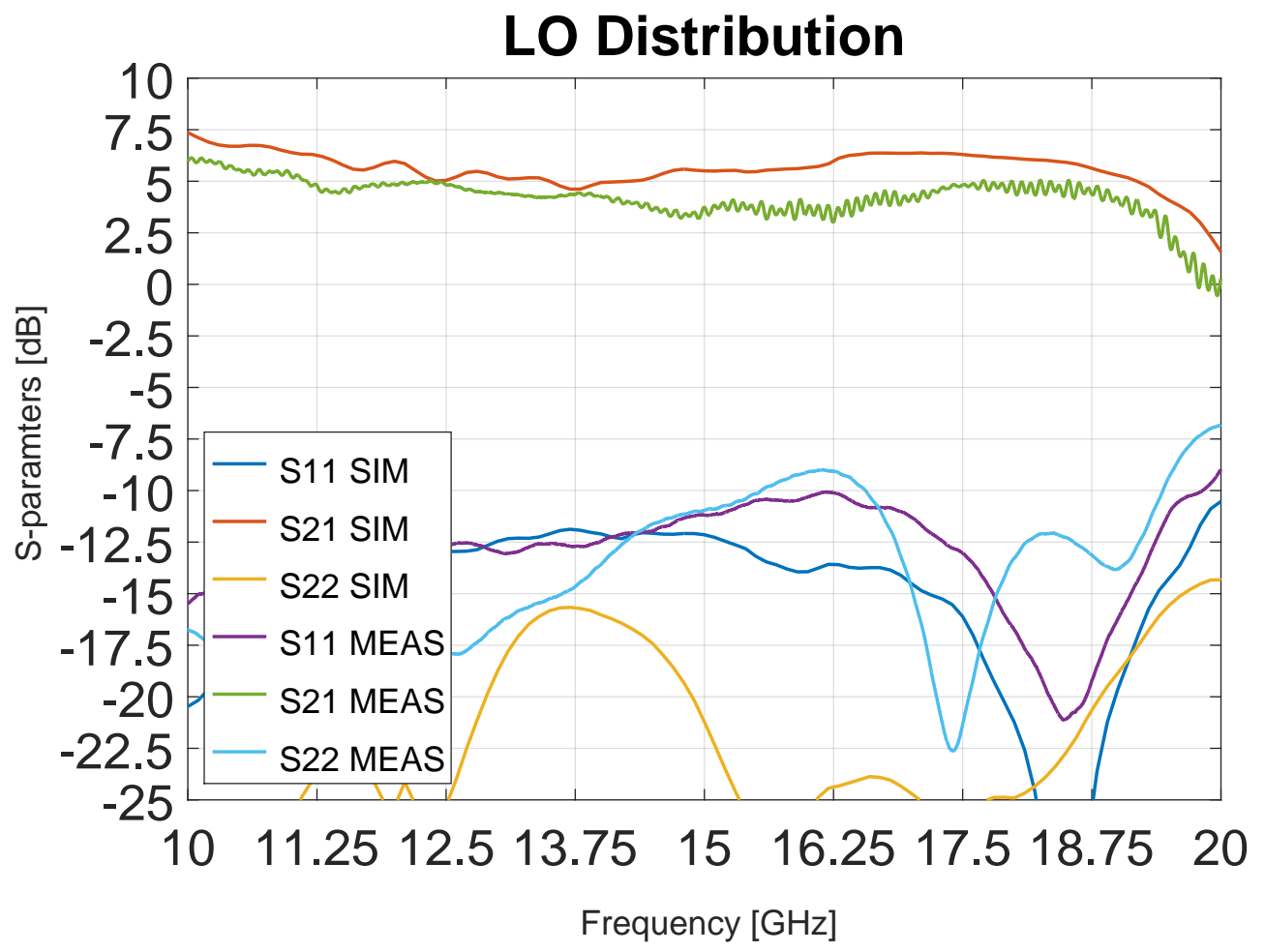

Figure 3.19: Comparison between the simulation and the measured results. For clarity reasons, only one path is depicted. It must be noted that the connectors are not included in the simulation.

The main function of the LO distribution board is to provide a coherent RF set of signals (same amplitude and phase) to the transmitters and receivers. Accordingly, differences in phase and amplitude must be explored, not being enough an image with the measurement and simulation of a single path. Fig 3.20 consists of three plots. A and B present the measurement of the amplitude and phase (respectively) of all 16 paths. C illustrates a zoom at $17 \mathrm{GHz}$ of the measurement of the phase of all 16 paths. As expected the presented channels have a very similar phase and amplitude. A maximum difference of $0.53 \mathrm{~dB}$ in the amplitude and $14.5^{\circ}$ in the phase are measured. These differences are due to tolerance errors in the $\mathrm{PCB}$ fabrication process, its components tolerances and solder- 
ing inhomogeneities. Nevertheless, these differences do not affect the performance of the system since a digital processing post calibration is applied in order to overcome the small disparities.
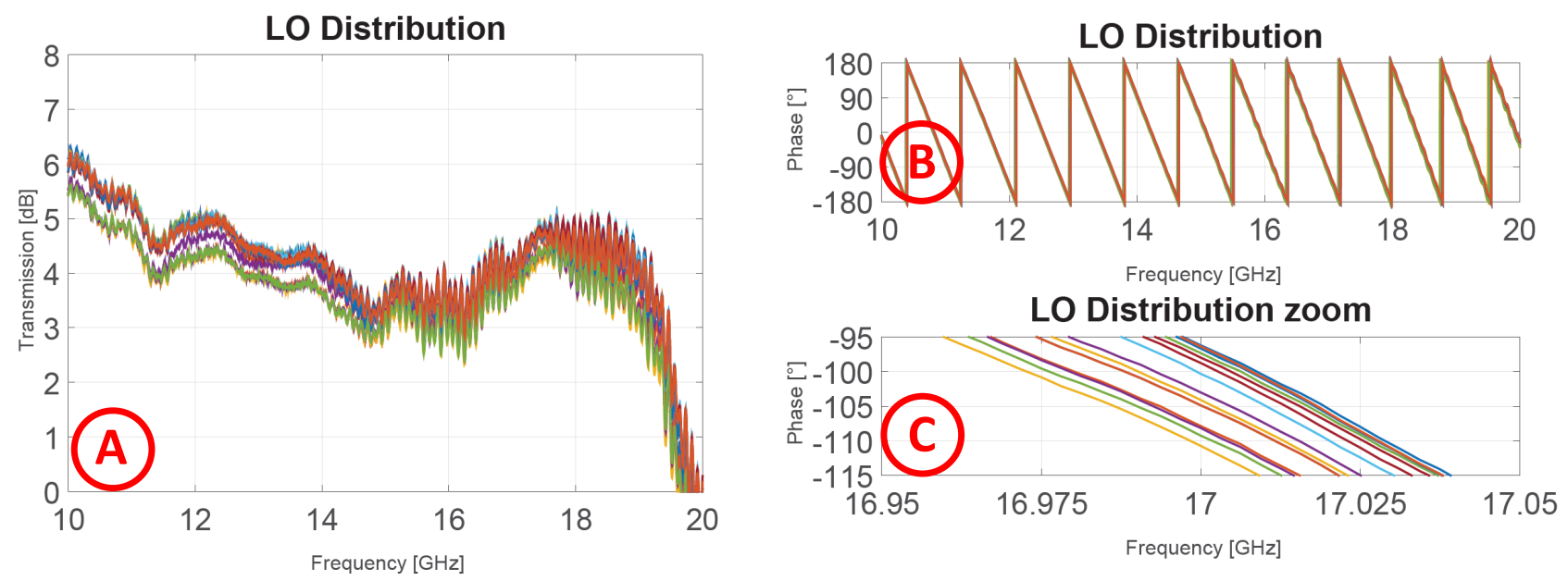

Figure 3.20: A. Measurement of the amplitude of all 16 paths from the input to the output of the $L O$ distribution board. B. Measurement of the phase of all 16 paths from the input to the output of the LO distribution board. C. Zoom at $17 \mathrm{GHz}$ of the measurement of the phase of all 16 paths from the input to the output of the $L O$ distribution board.

Table 3.2 shows the values of amplitude and phase at $17 \mathrm{GHz}$ of all paths. This table is very useful, because it provides accurate and tangible numbers. Moreover, it gives hints of how the real circuit works. For instance, paths 9, 10, 11 and 12 have a lower gain than the rest. It can be caused by a lower gain of the common amplifier that feeds these paths.

In this section, the LO distribution board design, fabrication and test have been fully described. Furthermore, an evaluation of the obtained results has been presented. As a conclusion, a LO distribution board, which is providing $16 \mathrm{RF}$ coherent signals, has been successfully measured and is ready to be integrated in the whole radar system. 


\begin{tabular}{ccccc} 
Path & Input port & Output port & Phase $\left[^{\circ}\right]$ & Amplitude $[\mathrm{dB}]$ \\
\hline 1 & 1 & 2 & -105.2 & 4.13 \\
2 & 1 & 3 & -104.9 & 4.03 \\
3 & 1 & 4 & -104.1 & 4.22 \\
4 & 1 & 5 & -103.0 & 4.08 \\
5 & 1 & 6 & -97.3 & 4.20 \\
6 & 1 & 7 & -96.6 & 4.15 \\
7 & 1 & 8 & -98.0 & 4.12 \\
8 & 1 & 9 & -96.2 & 4.05 \\
9 & 1 & 10 & -107.9 & 3.80 \\
10 & 1 & 11 & -110.7 & 3.72 \\
11 & 1 & 12 & -108.1 & 3.75 \\
12 & 1 & 13 & -109.2 & 3.73 \\
13 & 1 & 14 & -100.3 & 4.25 \\
14 & 1 & 15 & -98.8 & 4.22 \\
15 & 1 & 16 & -96.7 & 4.06 \\
16 & 1 & 17 & -96.4 & 4.09
\end{tabular}

Table 3.2: Measured values of all 16 paths at $17 \mathrm{GHz}$.

\subsection{Transmitter Board}

In this section the transmitter boards are introduced. Two transmitter boards are built in the radar system, consequently, two ports of the LO distribution board are connected with RF cables to the two inputs of the transmitters. A switch chain is responsible for the selection of the dedicated antenna at the proper time, according to the employed TDM scheme. This switching process is controlled by the firmware's procedures of the digital system by means of setting a Complex Programmable Logic Device (CPLD).

\subsubsection{Transmitter Board: System Level Considerations}

An analog procedure to the one of the LO distribution board is followed. First of all, an excell sheet with the link budget is completed as Fig. 3.21 depicts. In it, the sum of the produced signal gain by the amplifiers likewise the subtraction of losses produced by the connectors, switches and microstrip lines is calculated together to estimate the overall signal level at each stage of the chain. The noise figure, which represents the degradation of the signal-to-noise ratio in the RF chain, is also included in the excell block diagram. It is calculated following the equation (3.2). As described in previous section, the order of components, as well as OCP1 and TOI consideration are studied with this excell table before of the fabrication of the PCB. 


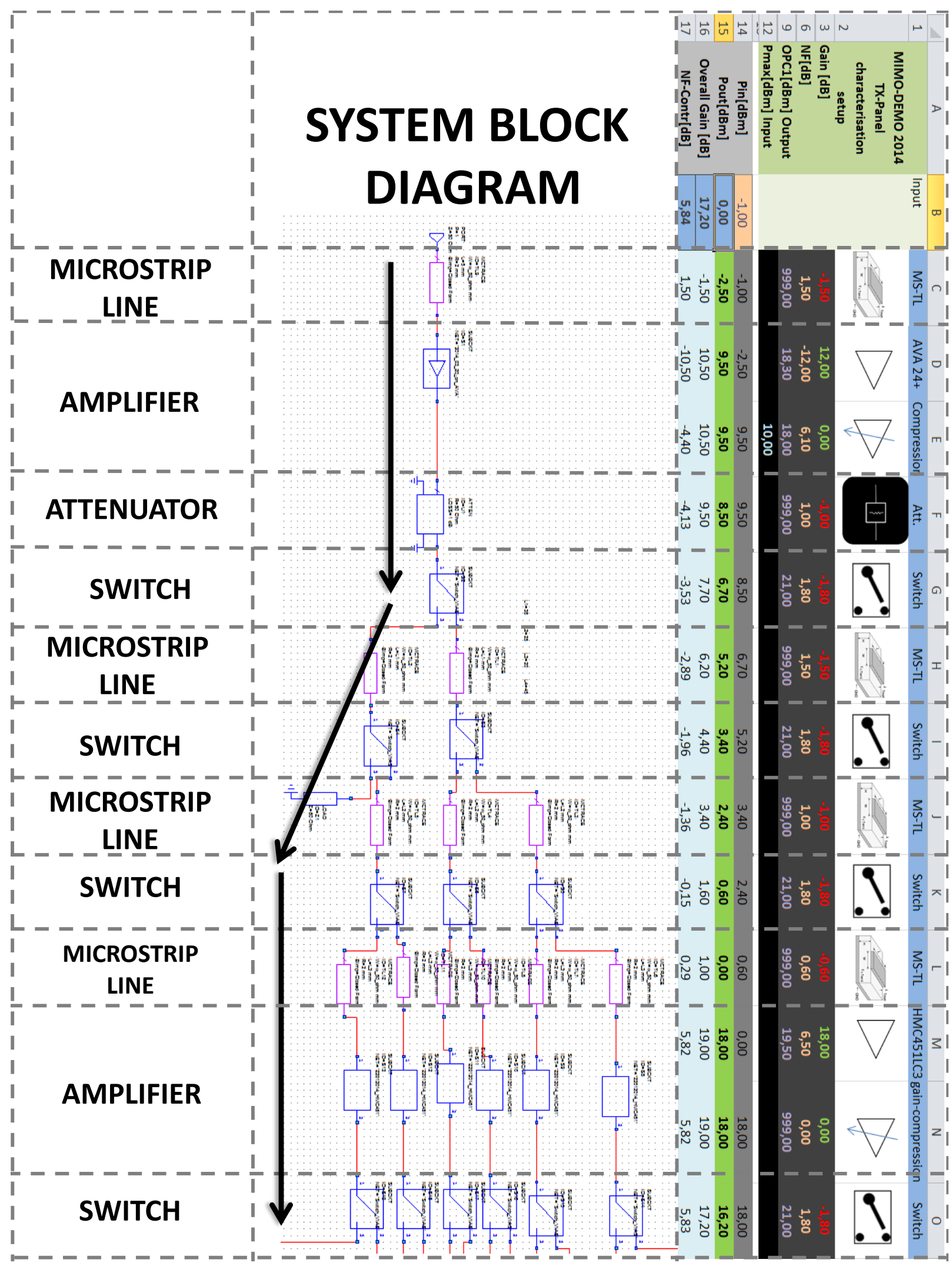

Figure 3.21: System block diagram used for simulation purposes (left-below) and estimated system power levels (right-above). 


\subsubsection{Transmitter Board: Simulation and Measurement}

Once a simulation was finished, the transmitter board layout was completed in Altium designer. It includes DC circuits in inner layers, DC connectors, a CPLD (which sets the right voltage levels at the switching in order to select the desired signal path), two test couplers (with them an accurate measurement of the power level before the antenna is performed) and TSA. The layer stack-up is depicted in Fig. 3.6. The FR4 layers on the bottom of the TSA are milled since in a slotted line the fields propagate on top, as well as on the bottom of the structure in contrast to a microstrip line (which is isolated with a ground plane). A well known SMA is integrated with the board. Fig. 3.22 illustrates a photography of the fabricated board. In it the main blocks are highlighted: the Input connector, the switch chain, the test couplers and the antennas.

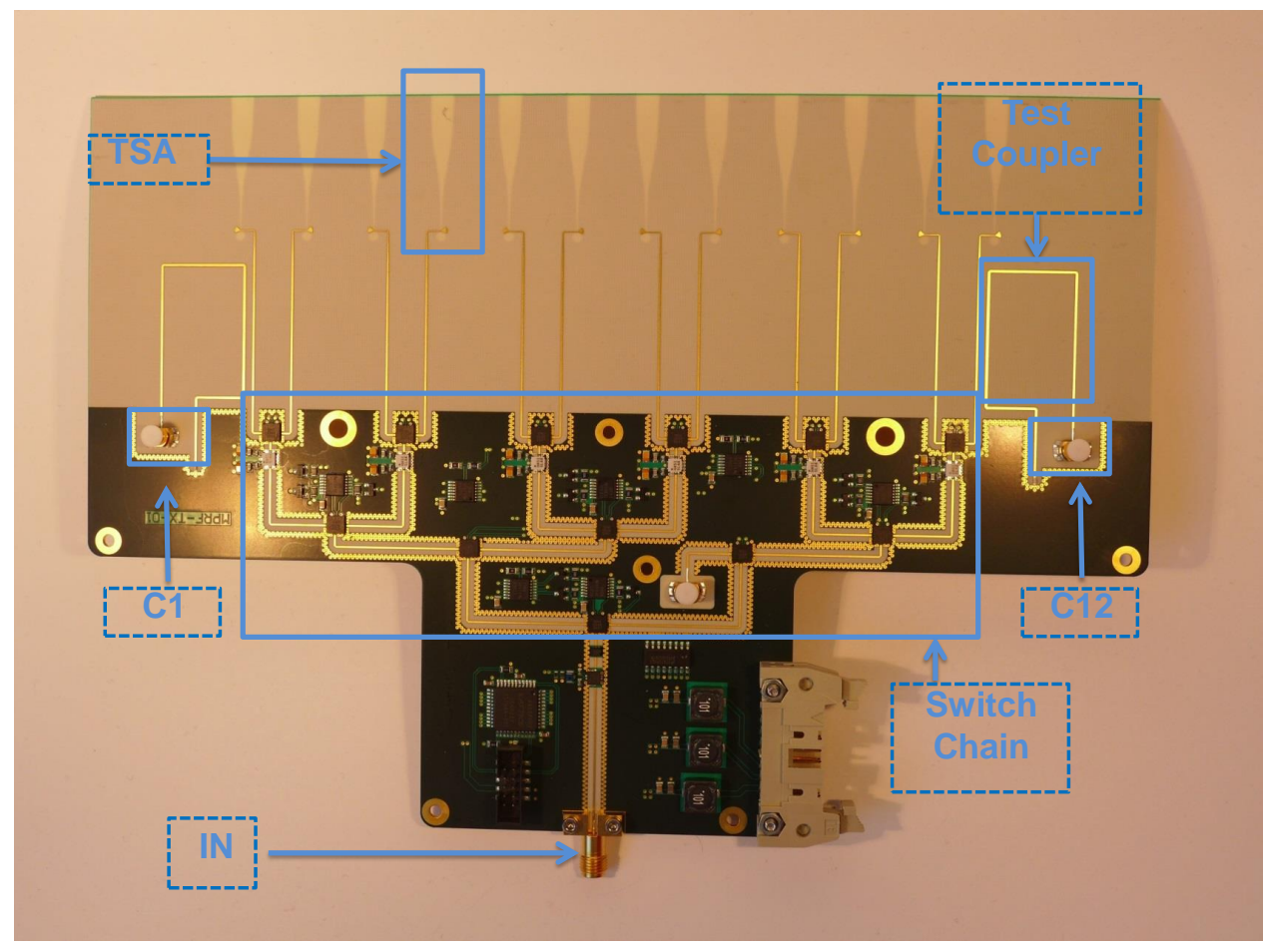

Figure 3.22: Photography of the fabricated transmitter board.

Fig. 3.23 introduces a comparison between simulated and measured S-parameters of the transmitter board. Considering that the the input and outputs (in the test couplers) have different connetors, a K-connector Short-Open-Load-Thru (SOLT) [15] calibration is performed with a commertial calibration kit. Moreover, the measurement includes the effect of a transition from the end-fire mini coaxial connector to K-connector. In addition, SMA connectors are mechanically connectible with K connectors. The measured results, in which the effect of the test coupler is de-embedded, are in agreement with the simulation, especially considering that the connectors are not included in the simulation. The 
estimation introduced in the system block diagram 3.21 is very helpful to provide a fast and relatively accurate approximation. The calculated overall gain in the block diagram is $17.2 \mathrm{~dB}$, the simulated $S_{21}=20.1 \mathrm{~dB}$ at $15 \mathrm{GHz}$ and the simulated $S_{21}=18.8 \mathrm{~dB}$ at $15 \mathrm{GHz}$. The differences between the simulation and the measurement are caused by not considering the connectors on the simulation. The input connector is an inexpensive SMA connector (which is not included in the simulation), consequently it worsens the input reflection. This facts explains the difference between measured and simulated return loss $S_{11}$.

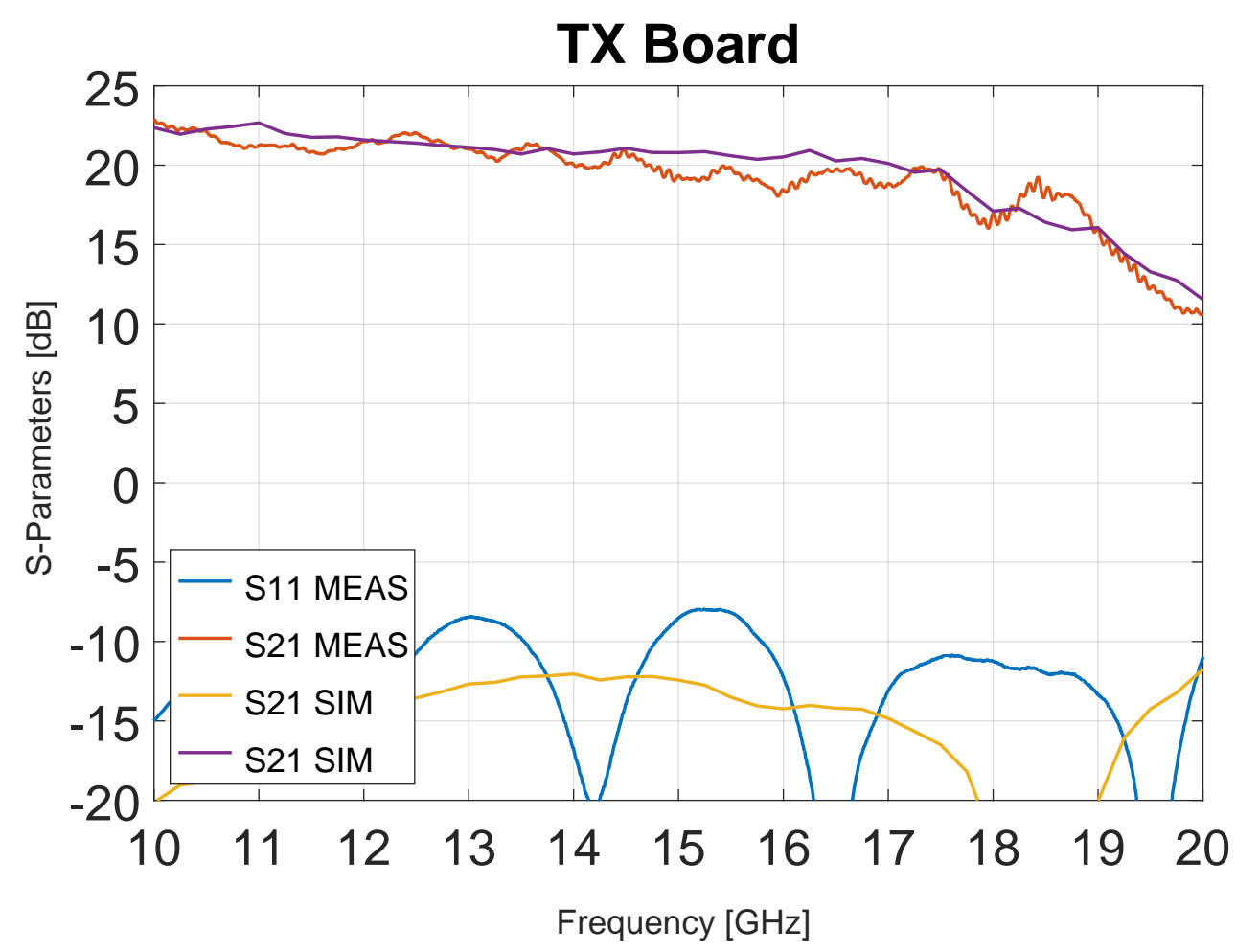

Figure 3.23: Comparison between the simulation and the measured results. It must be noted that the connectors are not included in the simulation.

The mutual coupling between antenna elements, in particular between TX and RX elements, is a well known and undesired effect of radar systems, especially for FMCW. When a signal is transmiited from a TX antenna, a part of the signal couples with an adjacent $\mathrm{RX}$ antenna and this may compromise the performance of the overall radar system. The maximum coupling between the antennas, with the chosen configuration for our system, is smaller than $C<26 \mathrm{~dB}$ in the frequency range of operation, as illustrated in Fig. 3.24 (left).

This undesired coupled power can saturate the mixer of the receiver. In order to avoid this malfunction, the maximum sustainable power at the receiver antenna would be 

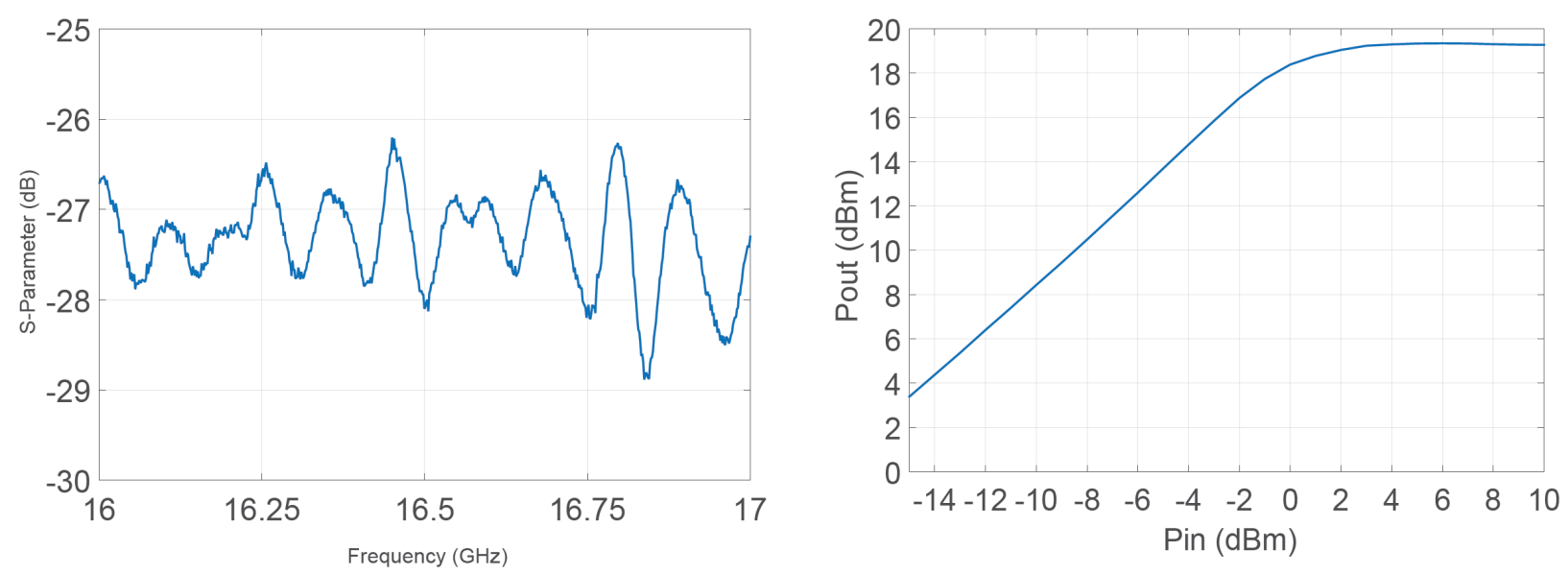

Figure 3.24: Measured maximum coupling between $R X$ and TX antennas (left). Measured input power versus output power in the transmitter board (right). The measurement was performed from the input to the coupler $C 1$ before the antenna. The effects of the coupler are compensated.

$P_{R X_{M A X}}=-5.5 \mathrm{dBm}$. Using the following equation:

$$
P_{T X_{O U T}}=P_{R X_{M A X}}-C
$$

a maximum transmit power of $P_{T X_{\text {OUT }}}=20.5 \mathrm{dBm}$ results. Fig. 3.24 (right) shows the measured input power versus output power with compensated effects of the test coupler in the transmitter board. As a conclusion, the coupling does neither damage nor affect the performance of our system because even if a strong signal is sent to the input of the receiver, the amplifiers at the transmit board saturate and will always provide a signal with a power below $P_{T X_{O U T}}<20.5 \mathrm{dBm}$.

In previous sections of this chapter, the system architecture, the MIMO array configuration and an overview of the RF frontend have been introduced. Moreover, a work flow, in which first a microstrip line model was developed and linked with measurements of building blocks was presented. In addition, a closer look to the LO distribution and transmitter board have been completed. More information about different aspects of the complete system can be found in [2]. In the following sections the assembly of the radar is described in detail. Furthermore some measures and discussion of the advantages of a MIMO radar are also included.

\subsection{Assembly of the RF system}

The RX, TX and LO distribution modules are built on panels, as shown in Fig. 3.25, which are stacked horizontally, in such a way that the desired antenna configuration is achieved. Each RX and TX panel structure is the result of the composition of a multilayer PCB, 


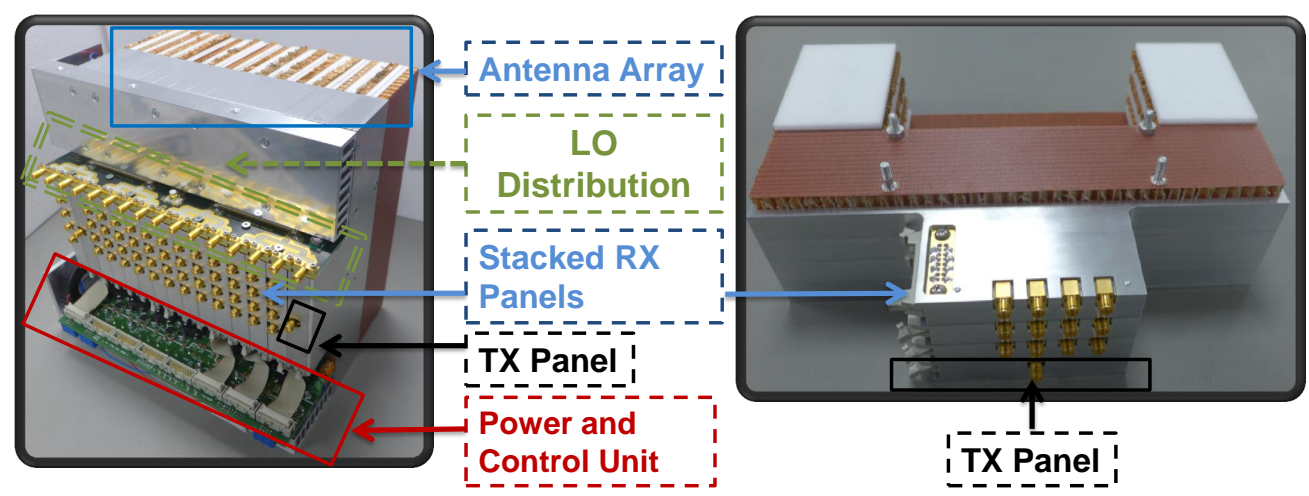

Figure 3.25: Photographies of the stacked TX, RX and LO panels. The left image shows the complete RF front-end unit. The right image is a section view of its structure.

stacked with a $7 \mathrm{~mm}$ thick honeycomb board, a $4 \mathrm{~mm}$ Rohacell foam stabilization system and a metal support.

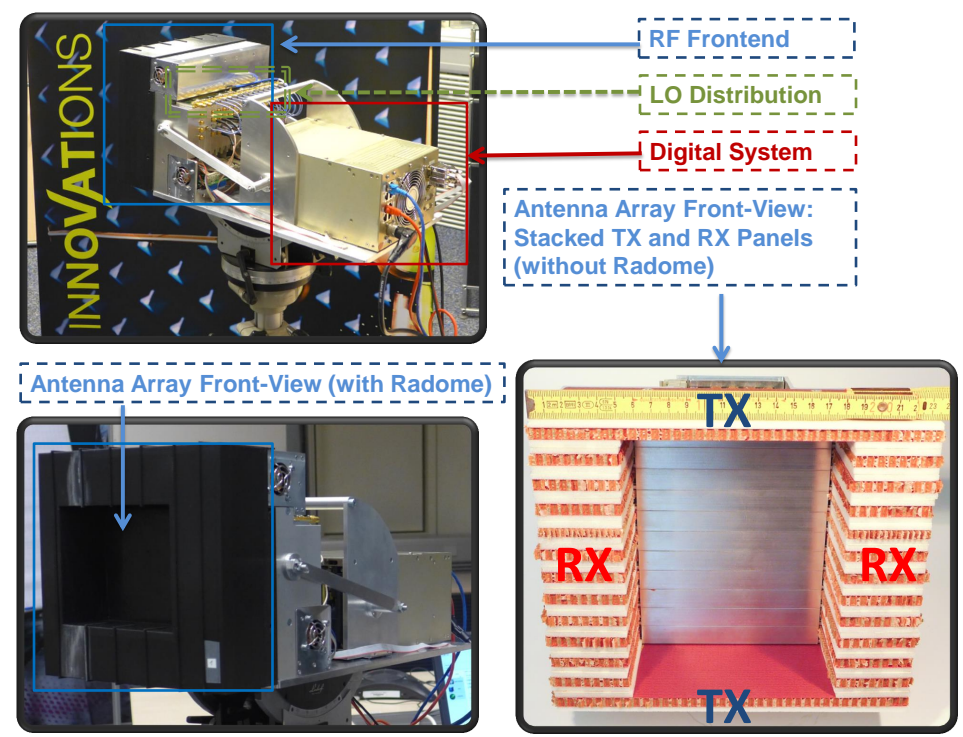

Figure 3.26: Photographies of the complete MIMO radar system. In the upper left image, the integrated RF front-end and digital system is presented. On the lower left and right images, a front view of the antenna array is shown.

In the upper left part of Fig. 3.26, the complete configuration of the $24 \times 24$ MIMO radar demonstrator is illustrated. In this part of the image, it is possible to see the assembled RF front-end and the digital system unit. The lower part of the figure shows the antenna array front view with the radome protection, on the left, and without the radome protection, on the right. In this lower right part of the image, the stacked RX and TX panels configuration can be seen front a front view. 


\subsection{System Performance and Image Results}

To demonstrate the radar performance and its capabilities in multi-target scenarios, a radar test field is set up with the use of corner reflectors. The test field, used for measuring the range and angular separation capabilities of the demonstrator, is shown in Fig. 3.27 (left). In this test setup, in order to carry out the free space measurements with the FMCW MIMO radar demonstrator, two corner reflectors at a range of $60 \mathrm{~m}$ with nominal radar cross section (RCS) of $36 \mathrm{~m}^{2}$ are placed in front of the radar at a slightly different orientation owing to differences in the measured RCS and different radial and lateral spacings. The persons shown behind the reflectors serve the purpose of representing the size of the image compared to the scenario.
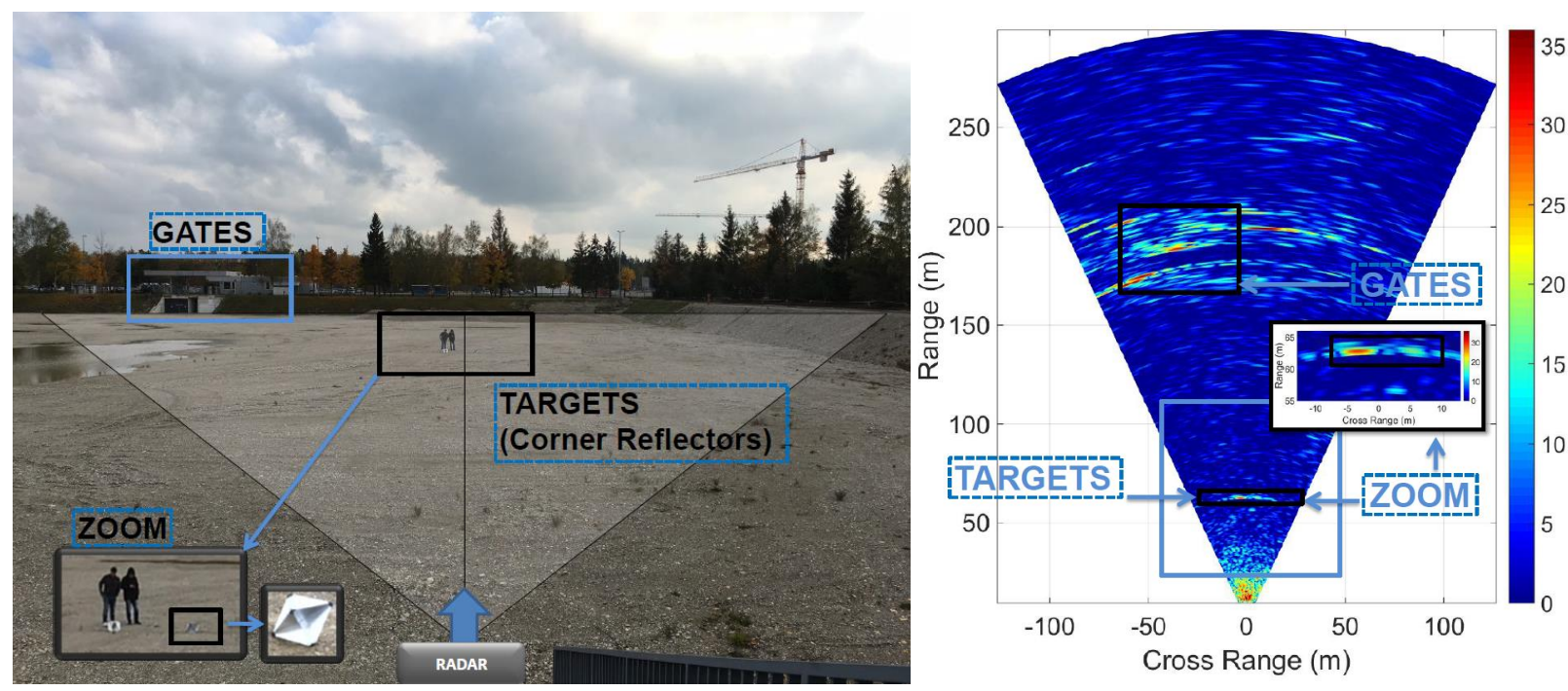

Figure 3.27: The MIMO radar test field with two corner reflectors at a range of $60 \mathrm{~m}$ with nominal radar cross section (RCS) of $36 \mathrm{~m}^{2}$ (left). Range-Azimuth section view of the 3D MIMO radar image capture from the radar setup scenario with two reflectors at a distance of $60 \mathrm{~m}$ (right).

Each of the images is generated with 24 up-ramp chirp signals ranging from $16 \mathrm{GHz}$ to $17 \mathrm{GHz}$ and with a duration of $100 \mu \mathrm{s}$. For easier understanding of the performance parameters, a slice of the $3 \mathrm{D}$ images is presented in the form of $2 \mathrm{D}$ image. A $2 \mathrm{D}$ radar image, cut in the azimuth plane, of the complete 3D image produced by a image reconstruction algorithm is shown in Fig. 3.27 (right). In this example, the reflectors are placed within the same range cell at a distance of $60 \mathrm{~m}$ and at an angular distance of $4^{\circ}$ (about $8 \mathrm{~m}$ lateral separation). It is possible to identify the two corner reflectors, together with additional reflections coming from two metal gates that were on the left at around $170 \mathrm{~m}$ distance from the radar, as shown in Fig. 3.27 (left). The zoom into the targets in this 
image, makes it is easier to clearly identify and separate the 2 corner reflectors at $60 \mathrm{~m}$ range. The amplitude of the target on the right is $6 \mathrm{~dB}$ lower due to a non identical alignment of the used corner reflectors.

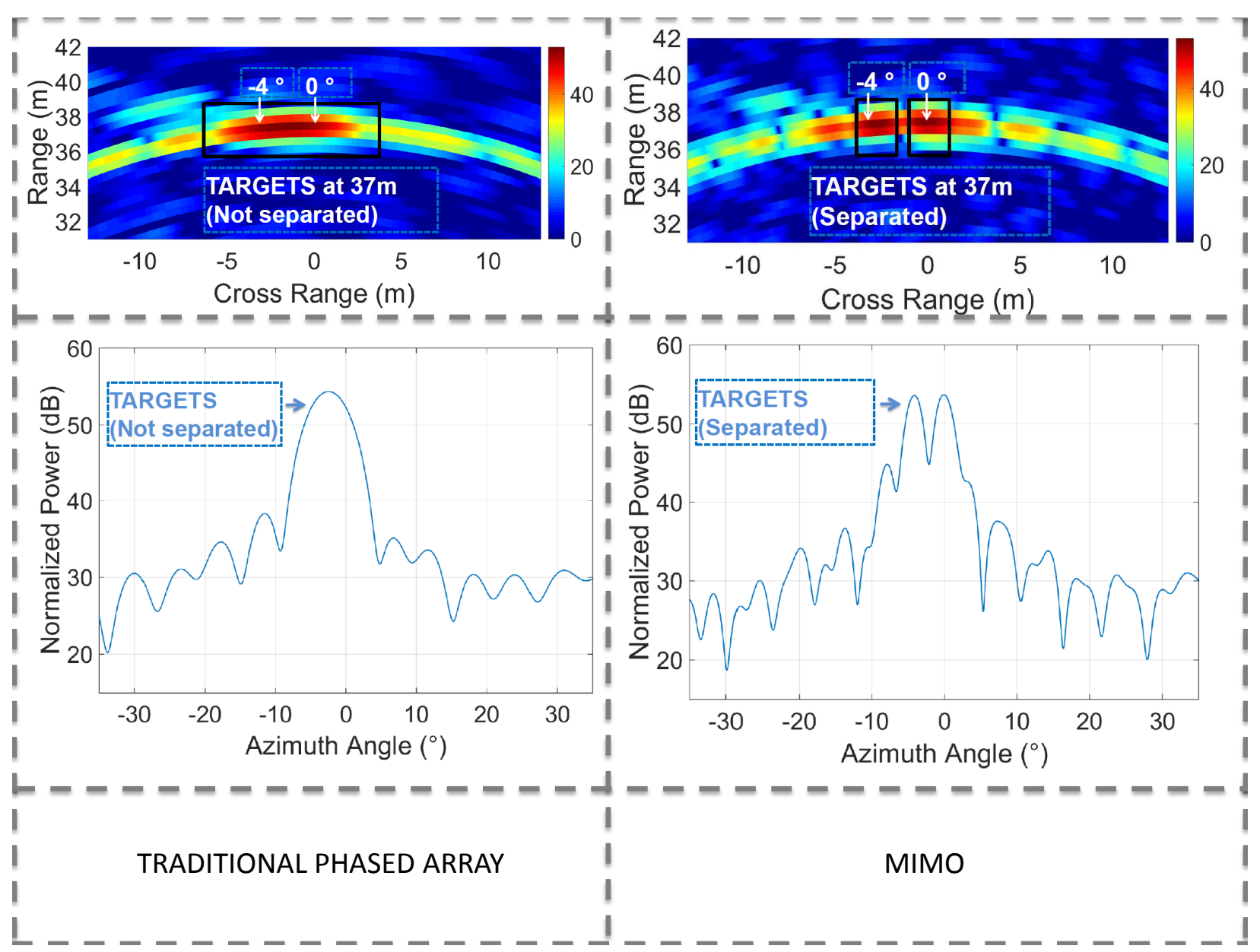

Figure 3.28: Angular separation capability test. On the left only one transmitter and traditional $D B F$ is used. On the right, a MIMO algorithm is used.

The angular separation capability, previously described in Fig. 3.2 and equation (3.1), is now examined within a range cell in a scenario based on two ideal point targets, similar to what is shown in Fig. 3.27 (left), with two corner reflectors with radar cross section of $36 \mathrm{~m}^{2}$, but now at a distance of $37 \mathrm{~m}$. The system's ability to resolve multiple target scenarios is mainly determined by the width of the main lobe of the antenna, after Digital Beam Forming (DBF) processing. As a result, the limiting factor for the angle separation capability is solely based on the width of the shaped lobes and the angular resolution is approximately expressed as in equation (3.1). Although interpolation of the missing data and discard of the redundant data are performed, differences between the measured angular resolution and the one given by equation (3.1) are expected to arise due to the 
fact that the equation is an approximation based on a limited number of antenna elements.

The processing of the collected data is carried out in two different ways. First, through conventional DBF by using only 1 transmit element and no virtual array with a traditional phased array approach and second, by using a MIMO processing approach. To determine the resolution limit, both reflectors are laterally moved toward each other, until the separation of the targets is no longer possible.

In Fig. 3.28 (left) the conventional processing is used. As depicted in this figure, only a single target can clearly be seen in the middle of the detection area of the sensor. A corresponding range cut gives the graph shown in the upper part of the image. Only one half-width main lobe is clearly visible, of $5.9^{\circ}$, which well matches the theoretical value determined by equation (3.1) of $5.79^{\circ}$ with the physical array size. Therefore, since the beam-width is greater than the angular distance of the targets, a separation of the targets is not possible without MIMO processing. Subsequently, the processing based on the MIMO virtual aperture is applied. The measured beam-width is $2.89^{\circ}$ a similar value to the theoretical one $\left(2.5^{\circ}\right)$. The results of the processing are shown in Fig. 3.28 (right). It can now be seen, with a closer look as shown in Fig. 3.28 (right), that the two close targets become uniquely identifiable. Both targets are clearly visible at a distance of approximately $4^{\circ}$.

\subsection{Final conclusions}

In this chapter a proof of concept of a complete MIMO radar is presented. It proves with measurements that the MIMO algorithm improves the resolution as theoretically expected. However, there are different aspects to improve. Even if the system is still portable, it is quite heavy (7 $\mathrm{kg}$ approx) and bulky $(55 \mathrm{~cm} \times 27 \mathrm{~cm} \times 27 \mathrm{~cm})$. The TSA antennas radiate in the direction perpendicular to the $\mathrm{PCB}$, accordingly multiple $\mathrm{PCBs}$ in a sandwich configuration are needed to form a 2D-antenna array. Therefore, the cost are also multiplied by the number of PCBs. In the next chapter, a new radar, with a different antenna technology that enables a 2D-antenna array in a single PCB is presented. 


\section{Bibliography}

[1] [Online]. Available: https://en.wikipedia.org/wiki/Technology_readiness_level

[2] A. Ganis, E. Miralles, B. Schoenlinner, U. Prechtel, A. Meusling, C. Heller, T. Spreng, J. Mietzner, C. Krimmer, B. Haeberle, M. Maier, S. Lutz, C. Weckerle, H. P. Feldle, M. Loghi, A. Belenguer, H. Esteban and V. Ziegler "A Portable 3D Imaging FMCW MIMO Radar Demonstrator with a Modular 24x24 Antenna Array" IEEE Trans. Microw. Theory Techn.

[3] R. Janaswamy, "Analysis of the tapered slot antenna", in IEEE Transactions on Antennas and Propagation, vol. 35, no. 9, pp. 1058-1065, Sep. 2003.

[4] M. Skolnik, "Radar Handbook, Third Edition", Electronics electrical engineering, McGraw-Hill Education, 2008.

[5] T. Spreng, S. Yuan, V. Valenta, H. Schumacher, U. Siart and V. Ziegler, "Wideband $120 \mathrm{GHz}$ to $140 \mathrm{GHz}$ MIMO radar: System design and imaging results", in Microwave Conference (EuMC), 2015 European, Paris, pp. 430-433, 7-10 Sep. 2015.

[6] D. F. Williams, C. M. Wang and U. Arz "An Optimal Multiline TRL Calibration Algorithm" Microwave Symposium Digest, 2003 IEEE MTT-S International, Philadelphia, PA, USA, 2003, pp. 1819-1822 vol. 3.

[7] J. S. Hong and M. J. Lancaster, "Microstrip Filters for RF / Microwave Applications, 2nd Edition", Wiley, February 2011.

[8] J. A. Shaw "Radiometry and the Friis transmission equation" American Journal of Physics,Am. J. Phys. 81, 33 (2013).

[9] E. Wilkinson "An n-way hybrid power divider".IEEE Trans. Microw. Theory Techn., vol.8, no. 1, pp. 116-118. Jan. 1960.

[10] [Online]. Available: https://en.wikipedia.org/wiki/RF_switch

[11] [Online]. Available: https://en.wikipedia.org/wiki/Link_budget

[12] [Online]. Available: https://en.wikipedia.org/wiki/Noise_figure 
[13] [Online]. Available: http://www.eetimes.com/document.asp?doc_id=1276328

[14] J. P. Dunsmore, "Handbook of Microwave Component Measurements: with Advanced VNA Techniques", Wiley, September 2012.

[15] J. Stenarson and K. Yhland, "Residual error models for the SOLT and SOLR VNA calibration algorithms", ARFTG Conference, 2007 69th, Honolulu, HI, 2007, pp. 1-7. 


\section{Chapter 4}

\section{A Compact Multifuctional MIMO Radar}

In chapter 3, a first MIMO radar demonstrator based on TDM scheme is presented. Even though this system is fully operational, demonstrates the MIMO principle and is adequate for some scenarios, it still has some drawbacks considering flying platforms as main application. The antennas used (in the first MIMO demonstrator) are TSA, they are capable of covering a broadband frequency band. In addition, they are easily integrable on a regular PCB substrate. However, if a 2D array is demanded, more than one PCB needs to be assembled in a sandwich configuration (see Fig. 3.25 and Fig. 3.26), since TSA radiate in the broadband direction to the PCB. This increases the cost and the size of the system.

In this chapter, a second iteration of our MIMO Radar is presented, which aims to be more compact and multi-functional. Here, a single PCB integrates a RF frontend with TX and RX antennas. Furthermore, the TSA are replaced by patch antennas [1], which typically are very narrow band antennas (around 5\% of relative bandwidth). A microstrip patch antenna can be fed in different ways: with a coaxial connector, which inner's core is connected to the patch at the $50 \Omega$ location, with a microstrip line directly connected to the patch at $50 \Omega$ location, or with a slot cut off on the ground of a microstrip line [2]. The last method has several advantages, as for instance the independent selection of antenna and feed substrate materials or the possibility to increase the operational bandwidth to $30 \%$.

Moreover, MIMO TX and RX radiating elements are placed in a rectangular configuration, the center of the antenna aperture is not occupied and is thus, available for different functionalities. A torch, a laser pointer, a gun or antennas for communication purposes can be placed occupying the space. The used object can be located in the phase center of the virtual array, in such a way that the same coordinate system is shared by both systems.

In this chapter, a 3D MIMO medium range imaging radar in combination with a communication antennas and a camera placed on the center of the MIMO array demonstrator 
is presented. The device has 36 radiating elements: $16 \mathrm{RX}$ antennas, $16 \mathrm{TX}$ antennas and 4 Spiral antennas for communication purposes. The radar operates in a frequency band from $16 \mathrm{GHz}$ to $17 \mathrm{GHz}$, this leads to an operation bandwidth of $1 \mathrm{GHz}$. The proposed system is an exemplary demonstration of a multifuctional system that integrates wireless communication and sensing functions, which can be used simultaneously. The wireless communication can be used as a telemetry link which triggers some modifications of the radar waveform, as for instance the ramp parameters, as well as for sending 3D radar images to a base station while the camera would give a real time image to an operator. The fabricated radar system is integrated with a novel 3D printed housing, which dissipate the heat of the circuitry, protects it and allows a very easy and straightforward assembly.

My contributions to this work are: the system design of the antenna board including MIMO array configuration, link budget and component selection, the design of the RF-key components as Resonant Slot Patch Antennas (RSPA) 3], new vertical (PCB face-to-face) throght Rogers and FR4 dielectric material transition and a 1 to 3 Wilkinson power splitter, complete system layout description (including a complex layer stack up), RF characterization of the antenna board.

\subsection{System Architecture}

The system architechture of the fabricated MIMO radar demonstrator is introduced in the form of a block diagram in Fig. 4.1. The 2 main elements can be seen: the RF frontend and the digital system.

The RF Frontend encloses the antenna board and the receiver board. The chirp signal generated by the DDS and PLL is sent to the antenna board. There, a 3 way Wilkinson divider is distributing the FMCW ramp to two switch chains and the receiver board. Correspondingly, each switch chain selects between 8 TX antennas or a matched load permitting a TDM configuration and thus orthogonality of the signals. The antenna board also includes 16 RX antennas, that simultaneously lead the reflected, and thus delayed chirp, to the receiver board. Afterwards, the 16 received signals are mixed with the originally sent chirp in order to obtain the beat frequency with the information of the distance to the target.

The modular approach enables to add and change extra hardware into the 3D printed housing. Accordingly, the orthogonality could also be obtained if two different signals were generated and transmitted through the corresponding two switch chains to two different antennas simultaneously. In such a way, TDM approach would be avoided and a real time MIMO radar could be built. The system processing is carried out on a laptop, where a graphic Human-Machine Interface (HMI) on the system processing workstation configures the Direct Digital Synthesizer (DDS) and Phase-Locked Loop (PLL) with the desired 


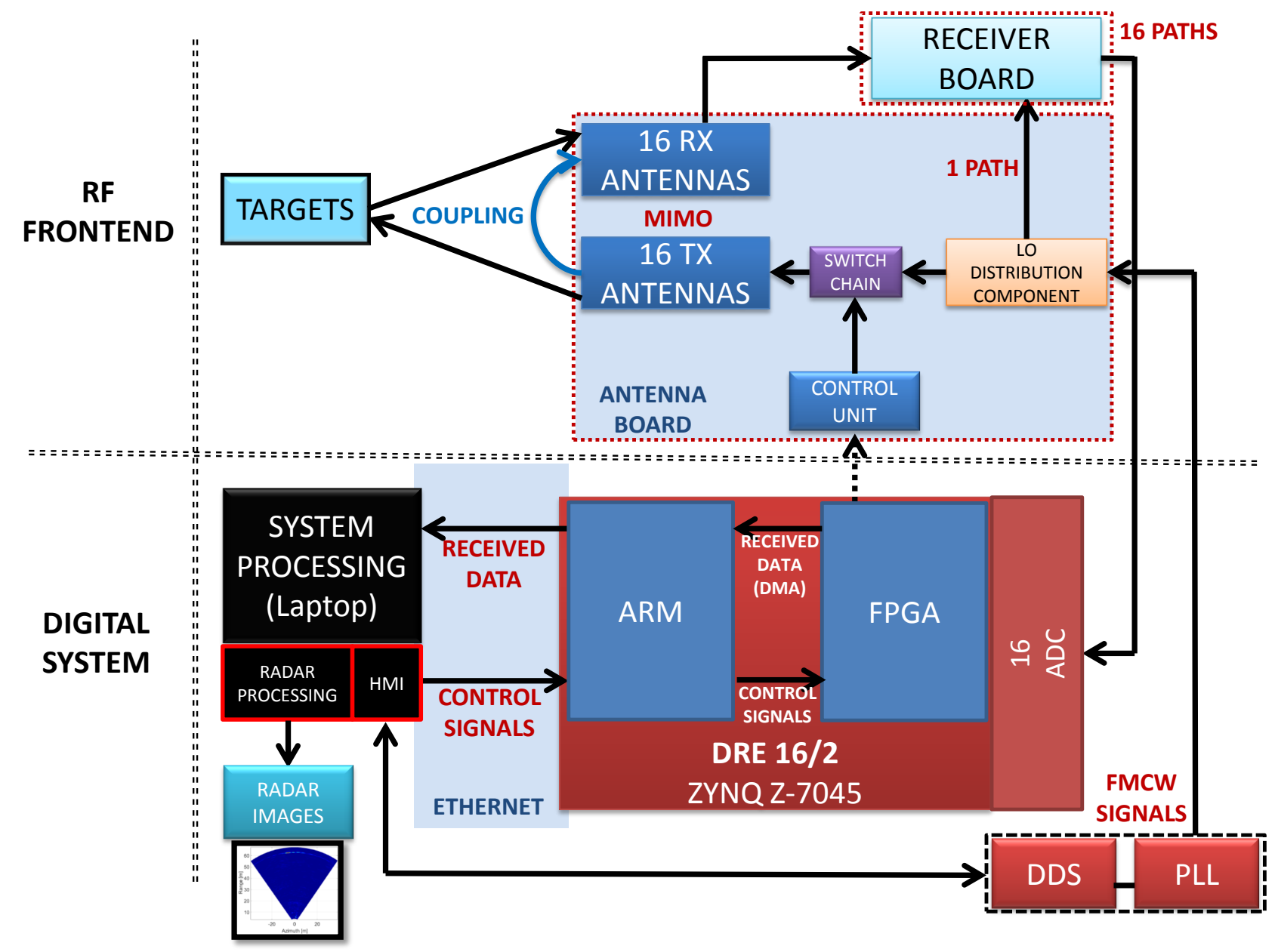

Figure 4.1: Block diagram of the 3D Imaging FMCW 16x16 MIMO RADAR Demonstrator. The upper and lower part of the image illustrate the RF front-end and the digital system blocks, respectively.

FMCW chirp generation parameters. The control unit sets the proper bit combination in order to select the proper antenna and MIMO radar processing of the received signals. A ZYNQ Z-7045 platform which includes a single Field Programmable Gate Array (FPGA) and an Advanced RISC (Reduced Instruction Set Computer) Machines (ARM) serves as an interface between the ADC and the laptop preforming the acquisition of digital data. After that the pre-processed data is sent to the offline software based radar signal processing functions on the workstation that applies digital beam-forming techniques to generate the final radar images. 


\subsection{MIMO Array Configuration}

As previously introduced, MIMO radar consists of two set of TX and RX radiating elements generating a partially filled array. Each combination of RX and TX antennas generates a orthogonal data stream thanks to the TDM approach. As Fig. 4.2 illustrates, the equivalent virtual array (in purple) can be calculated as the discrete convolution of the overall RX (in red) and TX (in blue) element positions.
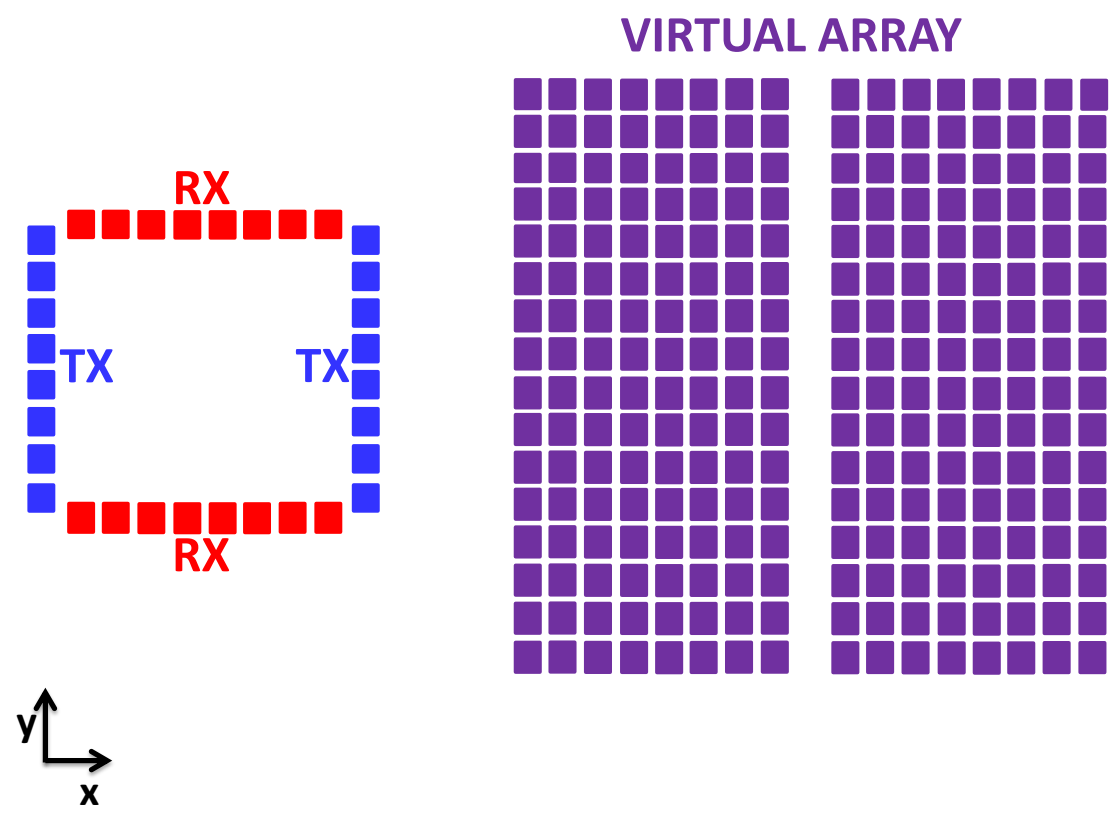

Figure 4.2: Schematic representation of the MIMO antenna configuration with the physical array (left) showing the $16 \mathrm{TX}$ antennas in blue, the $16 \mathrm{RX}$ antennas in red and the resulting virtual array (right).

Fig. 4.3 shows the position of the radiating element's phase centers and the corresponding MIMO virtual array (the discrete convolution of the RX and TX elements). The TX and RX radiating elements are placed forming a rectangle, in such a way the equivalent virtual array has four times the surface of a fully populated array with the same perimeter. This leads to an improvement in the resolution of a factor of two in each axis. In this configuration a free row appears. Some authors overcame this difficulty from the signal processing side by linear interpolating the missing values with very good results [4]. In contrast to the MIMO configuration of chapter 3, in which the sandwich configuration was too rigid, the resultant virtual array doesn't have redundant elements. This is achieved by displacing $\lambda / 4$ the RX antennas (see 4.3). Theoretically, the empty row can be suppressed by displacing $\lambda / 4$ the TX antennas towards the center of the array. This is not possible since the TX and RX patch antennas would have physical contact. 
The TX antenna element is a 2 patch array unlike the RX antenna element, which is a 4 patch array. In such a manner, the TX element size is reduced. Thus, 8 TX element fits in a single row and 8 RX elements plus 2 TX elements fit in a single column covering $160 \times 120 \mathrm{~mm}$ of the antenna aperture. Equations 4.1 and 4.2 calculate the angular resolution $\Delta \theta_{3 d B_{x}}$ in azimuth and $\Delta \theta_{3 d B_{y}}$ in elevation of the virtual array.
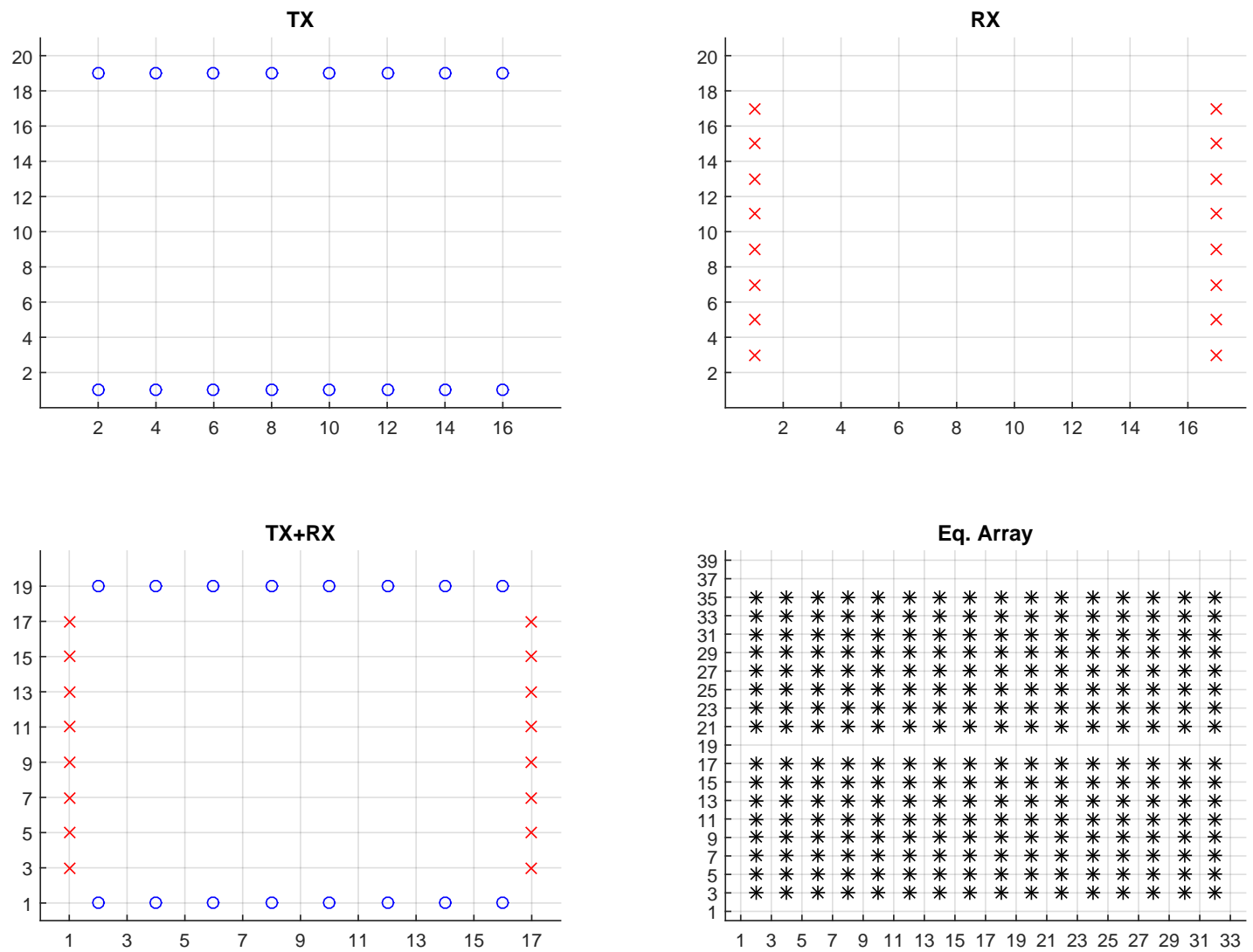

Figure 4.3: $R X$ array in red, TX array in blue and virtual array in black. A unit in the graph is equivalent to $\frac{d_{x}}{2}$ and $\frac{d_{y}}{2}$ respectively.

$$
\begin{aligned}
& \Delta \theta_{3 d B_{x}} \approx 50^{\circ} \frac{\lambda_{0}}{d_{x} N_{\text {virtual }}} \approx 50^{\circ} \frac{\lambda_{0}}{d_{x} 2 N_{\text {populated }}} \approx 3.7^{\circ} \\
& \Delta \theta_{3 d B_{y}} \approx 50^{\circ} \frac{\lambda_{0}}{d_{y} N_{\text {virtual }}} \approx 50^{\circ} \frac{\lambda_{0}}{d_{y} 2 N_{\text {populated }}} \approx 4.9^{\circ}
\end{aligned}
$$



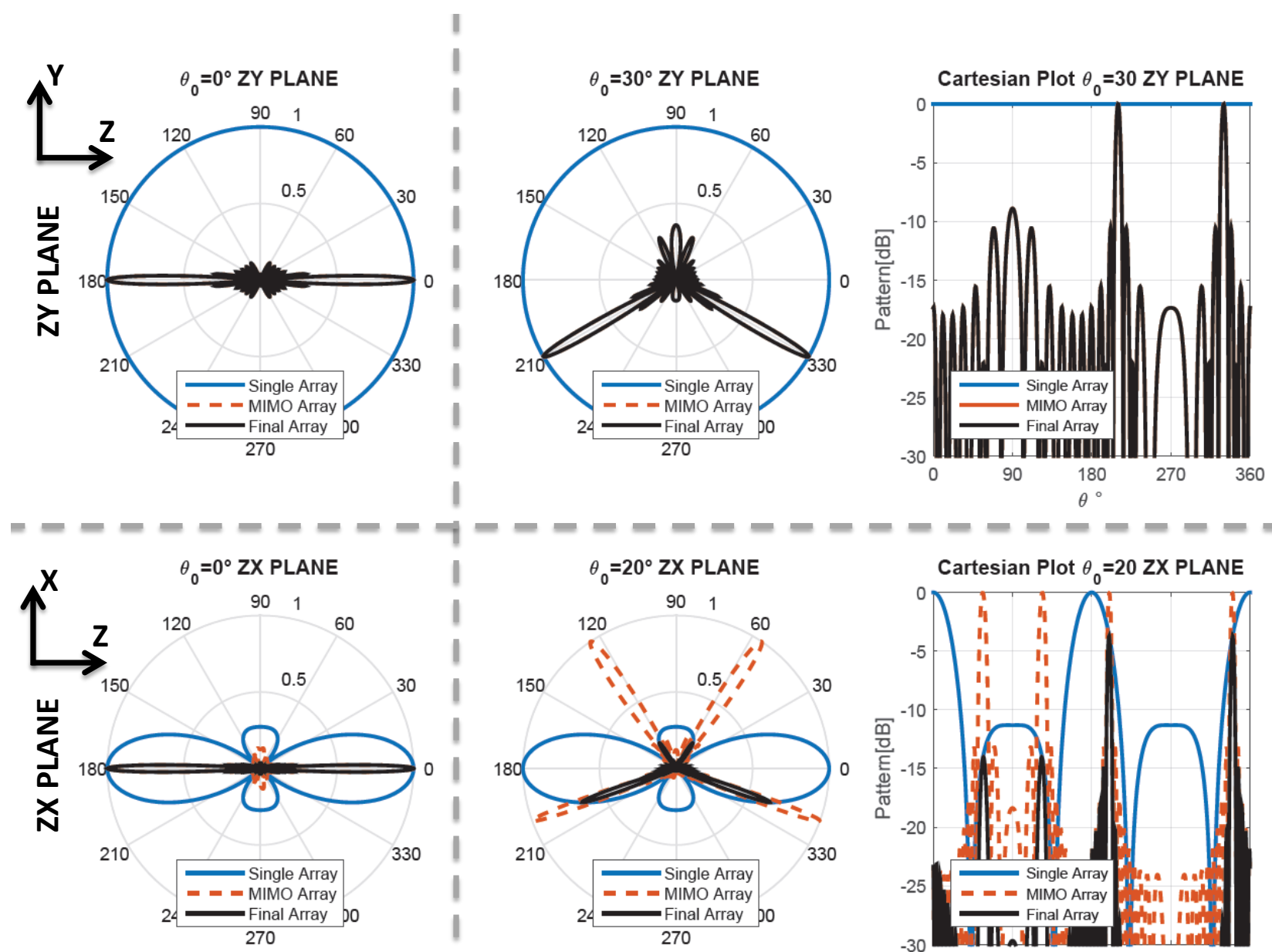

NO BEAM STEERING

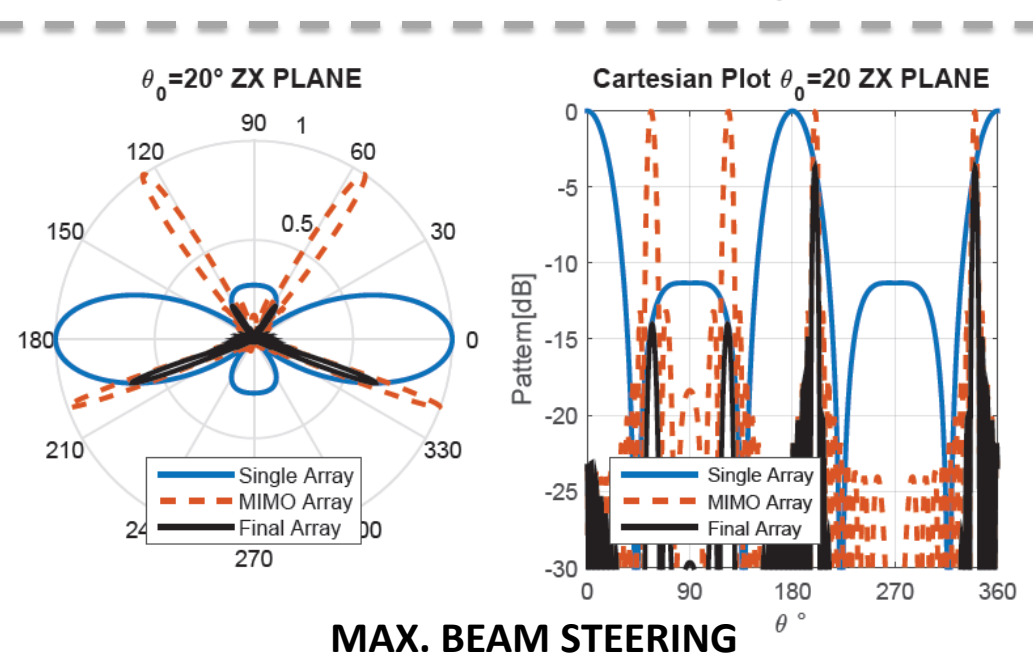

Figure 4.4: Virtual array pattern. Single element in blue. MIMO array in red. Multiplication of both in black. In some graphs, the black line overlaps with the red one.

with $\lambda_{0}$ being the wavelength, $N_{\text {virtual }}$ the number of virtual elements in either x- or y-direction, $N_{\text {populated }}$ the number of elements of an equivalent fully populated array with the same perimeter as our physical array and $d_{x}$ and $d_{y}$ the distances between elements. Regarding the empty row which appears in the center of Fig. 4.2, an interpolation of the data is performed, according to what has been previously described in [4]. $d_{x}$ and $d_{y}$ are 16 $\mathrm{mm}$ and $12 \mathrm{~mm}$, respectively, despite the fact that $\frac{\lambda_{0}}{2}=9.25 \mathrm{~mm}$ for the central frequency of operation. As a consequence of this, grating lobes appear. This fact was considered in the antenna array design.

Each plot of Fig. 4.4 displays three curves. First, the pattern of the receiver antenna element $F$ (blue curve), which is approximated by an array with four omnidirectional radiating elements, is shown. This array is fixed, therefore, phase control for each antenna 
element is not possible. Second, the pattern of the virtual array considering the middle row being empty and without applying any interpolation ( $A$ in equation 4.3) is depicted in red color. It must be noted that $d_{1}, d_{2}$ and $d_{k}$ are the radiating element's position in equation 4.3. Third, the combination of both, element pattern and cirtual array factor, $F_{t o t}$, following the array pattern multiplication property accordingly equation (4.4) is displayed in black color.

$$
\begin{gathered}
A=a_{0} e^{j k d_{1}}+a_{1} e^{j k d_{2}}+\ldots+a_{k} e^{j k d_{k}}+\ldots \\
F_{\text {tot }}=A F
\end{gathered}
$$

Fig. 4.4 shows six graphs. The first row is dedicated to the ZY cutting-plane, while the second one is dedicated to the ZX cutting-plane. In the first column, the antenna pattern is displayed without any beamsteering. The second column shows the antenna pattern with the maximum beam steering $\theta_{0}$ designed. The last column repeats the second column, but in cartesian coordinates and in $\mathrm{dB}$ units. Note that in the actual implementation a single element is not isotropic, which restricts the maximum beam steering of the radar as well as reduces the impact of the grating lobes (second column XZ cutting-plane).

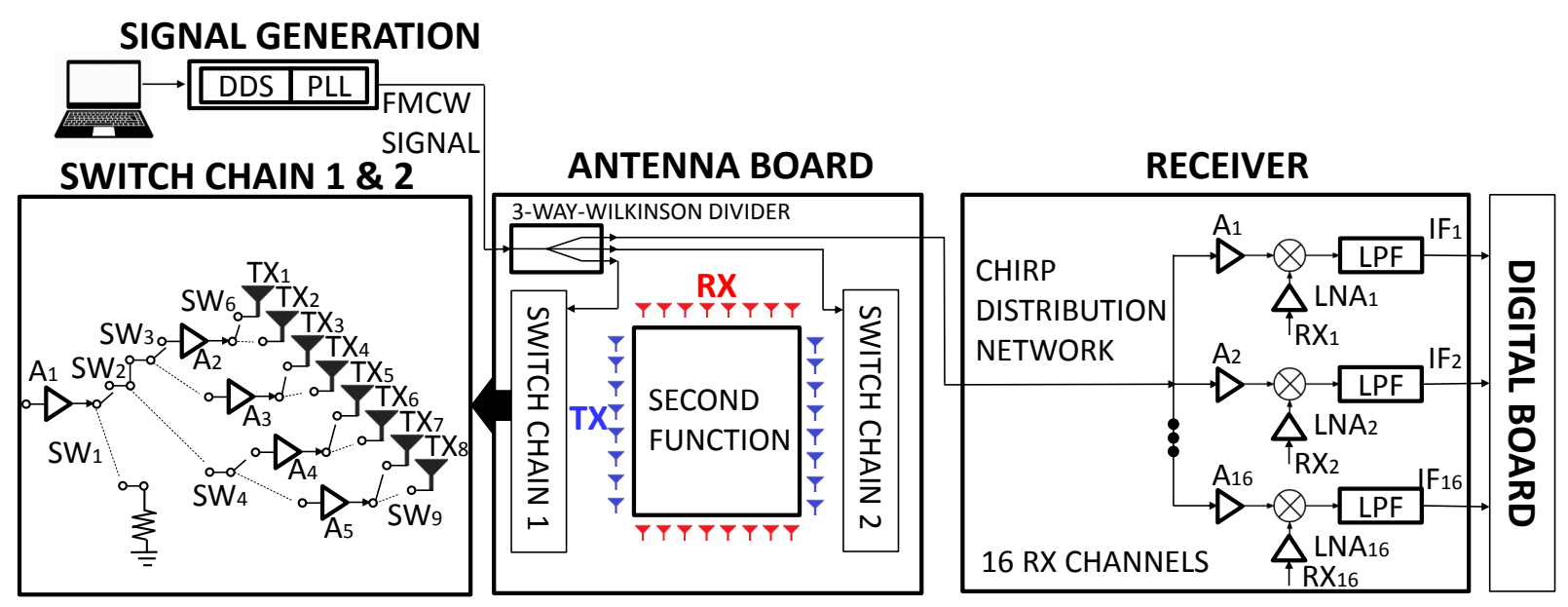

Figure 4.5: Schmatic diagram of the designed hardware.

\subsection{The RF Frontend}

The main blocks of the radar hardware are the antenna board, the receiver board and the signal generation. The circuit level block diagrams are shown in Fig. 4.5. 
The antenna board includes in a single planar PCB, 32 radiating elements, 16 for transmit TX (in blue) and 16 for receive RX (in red). They are placed in a rectangular configuration, in such a manner, that an unoccupied surface at the center of the array arises. In order to achieve the desired operational bandwidth and the desired range resolution, RSPA instead of traditional patch antennas are incorporated [3]. A three way Wilkinson divider equally distributes the FMCW ramp, that is generated by the DDS and PLL, to the two independent switch chains and the receiver. The two switch chains are able to select the proper antenna at the right time or to lead the signal to a terminated port (50 $\Omega$ resistor) in order to accomplish a orthogonality of the received data streams

under a TDM paradigm. A more detailed description of the antenna board can be found in [17].

The receiver board consists of 16 direct-conversion receivers. The RX signals are amplified by LNA and then fed to the mixers. A chirp distribution network splits up the chirp signal and distributes it coherently to all 16 stages. Each stage has its own amplifier in order to drive the LO input port of the mixer. The RX signal is then mixed with the originally sent chirp and low-pass filtered. From the output of the receiver, the IF signals go directly to the digital board.

The main elements of the digital board are the ZYNQ, which is a FPGA and an ARM processor in one chip, two 8-channel 14-bit ADC, which run at $100 \mathrm{MHz}$, and an Ethernet interface.

\subsection{RF Components Design}

In this section some RF components are introduced. The amplifiers, RF switches or the Microstrip line model are already presented in chapter 3 and therefore not presented again in this chapter.

\subsubsection{Resonant Slot Patch Antennas}

Microstrip antennas revolutionized the antenna field. They are lightweight, small and easily integrable with IC's on a PCB. In such a manner, mass production at low cost is possible. These antennas are widely utilized for defense and commercial applications and can replace many conventional antennas. Classical patch antennas cover typically a $5 \%$ of relative bandwidth [1], this would restrict the bandwidth of the FMCW ramp and lead to losses in the performance in terms of range resolution. A microstrip patch antenna can be fed in different ways: with a coaxial connector, which inner's core is connected to the patch at the $50 \Omega$ location, with a microstrip line directly connected to the patch at $50 \Omega$ location, or with a slot cut off on the ground of a microstrip line [2]. The last method has 
several advantages, as for instance the independent selection of antenna and feed substrate materials or the possibility to increase the operational bandwidth. If a proper size of the coupling slot is chosen, it also resonates, in such a way that the operational bandwidth of the antenna increases. However, the required coupling aperture also produces back radiation, although the coupling aperture is very small and the produced back radiation is much smaller than the forward radiation. More substrate layers with its corresponding patches can be added, in such a way, the bandwidth performance is enhanced, while the efficiency of the antenna diminishes and surface waves are excited on the PCB. Another way to enhance the bandwidth of a patch antenna is selecting a thick substrate with low $\epsilon_{r}[5]$.

\section{Single patch}
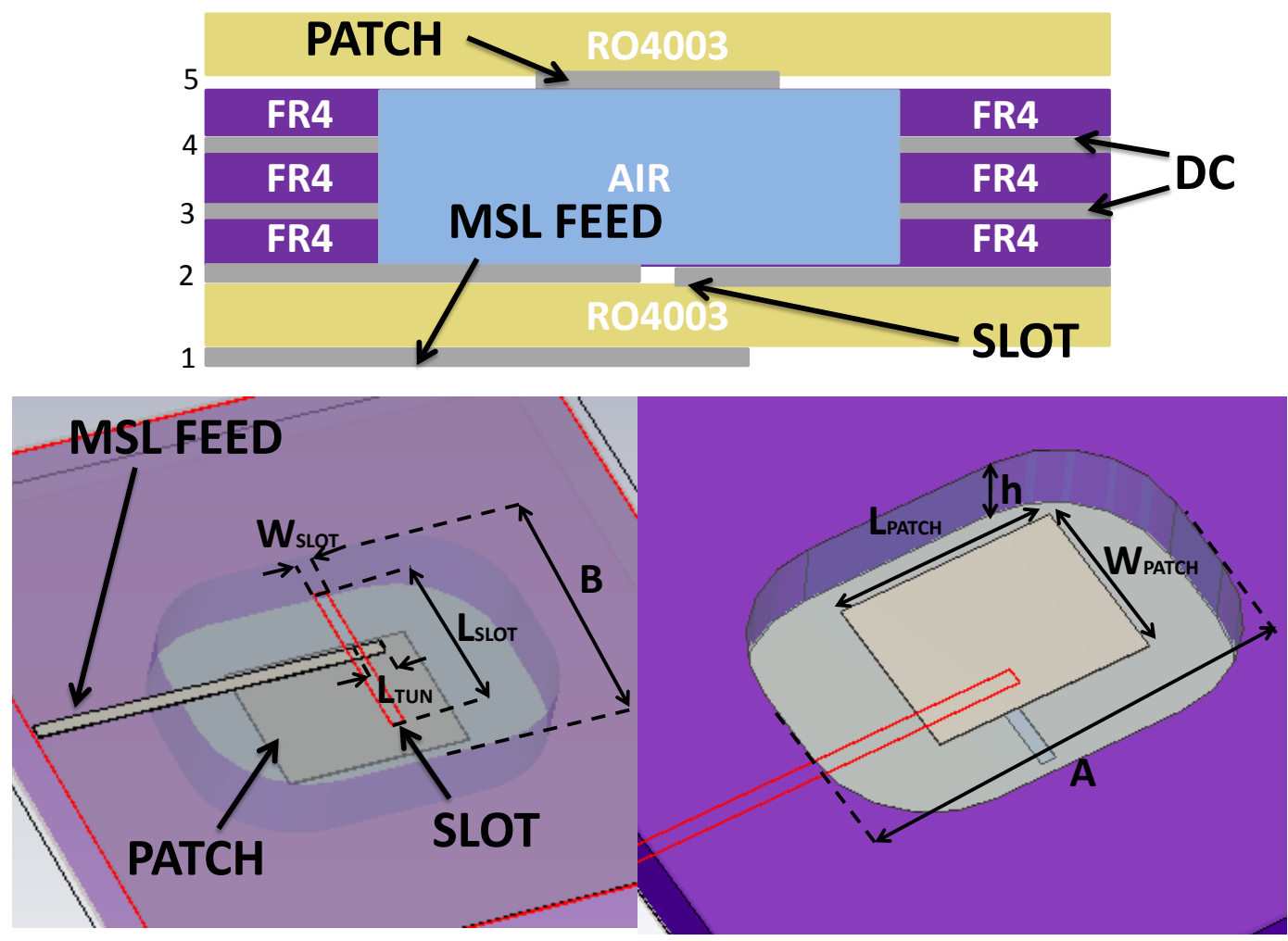

Figure 4.6: Antenna stack-up (top). 3D view of the fabricated antenna with its main design parameters (bottom).

Fig. 4.6 illustrates the selected antenna stack-up (top) and a 3D view of the antenna with its main design parameters (bottom). Layers 1 and 2 are filled with a RO4003 substrate with $\epsilon_{r}=3.55$ and height $h_{1}$ of $0.203 \mathrm{~mm}$. Between layers 2 and 5 there are several layers filled with an inexpensive FR4 substrate, an air filled cavity (with dimen- 
sions $\mathrm{A}=11 \mathrm{~mm}$ and $\mathrm{B}=8 \mathrm{~mm}$ ) is milled in those layers in order to create a low $\epsilon_{r_{A I R}}$ (equal to 1 in air) region between the slot (layer 2) and the patch (layer 5). The size of the cavity should be large enough to avoid strong electromagnetic interaction with the FR4 substrate, its $\mathrm{RF}$ material characteristics $\tan (\delta)$ and $\epsilon_{r_{F R 4}}$ are not suitable for RF circuits, since they are not stable within the same batch and the $\tan (\delta)$ is too high. The distance between the layer 2 and 5 is $h$ is $2 \mathrm{~mm}$. The patch is placed on top of the air gap, it is stacked to a RO4003 substrate with $\epsilon_{r}=3.55$ and height $h_{1}$ of $0.203 \mathrm{~mm}$, it is settled underneath the last substrate, in such a manner it is not accessible from outside once the PCB is stacked together. The size of the patch is $L_{P A T C H}=5.6 \mathrm{~mm}$ and $W_{P A T C H}=4.25 \mathrm{~mm}$. The thickness of all metal layer is $t=0.035 \mathrm{~mm}$. The coupling slot on layer 2 has a width $W_{S L O T}$ of $0.4 \mathrm{~mm}$ and a length $L_{S L O T}$ of $5.1 \mathrm{~mm}$. On the next layer (layer 1) a $50 \Omega$ microstrip line with width $W_{M S}$ of $0.442 \mathrm{~mm}$ is placed over the slot. The microstrip line stub has a length of $L_{T U N}$ of $0.7 \mathrm{~mm}$ from the center of the slot to a open circuit (see Fig. 4.6).

The presented antenna involves a lot of design parameters, these are the basic trends with the variation of these parameters [6]:

- Antenna substrate dielectric constant $\left(\epsilon_{r_{A I R}}\right)$ : The lower the permittivity, the wider the impedance bandwidth and the lower the surface wave excitation.

- Antenna substrate height (h): A thicker substrate (air gap in this case) leads to wider bandwidth, but less coupling for a determinate slot size.

- Microstrip patch length $\left(L_{P A T C H}\right)$ : It determines the resonant frequency of the antenna.

- Microstrip patch width $\left(W_{P A T C H}\right)$ : The wider patch, the lower resonant resistance of the antenna. It must be noted that square patchs have a very high cross polarization levels, and therefore must be avoided unless a circular or dual polarization is desired.

- Microstrip feed dielectric constant $\left(\epsilon_{r}\right)$ : It is independent of the antenna performance as long as the microstrip line has good transmission line properties.

- Microstrip feed dielectric thickness $\left(h_{1}\right)$ : It is independent of the antenna performance as long as the microstrip line has good transmission line properties.

- Slot length $\left(L_{S L O T}\right)$ : It affects the coupling level to the patch antenna, the second resonance frequency and the back radiation level.

- Slot width $\left(W_{S L O T}\right)$ : It affects the coupling level, but to a much less degree than $L_{S L O T}$. A typical ratio $W_{S L O T} / L_{S L O T}$ is $1 / 10$.

- Microstrip feed line width $\left(W_{M S}\right)$ : It controls the impedance of the microstrip line. It varies the coupling from the microstrip line to the slot. 
- Length of tuning stub $\left(L_{T U N}\right)$ : It must be slightly less than $\lambda_{g} / 4$. Shortening this length, the impedance locus moves in the capacitive direction on the Smith chart.

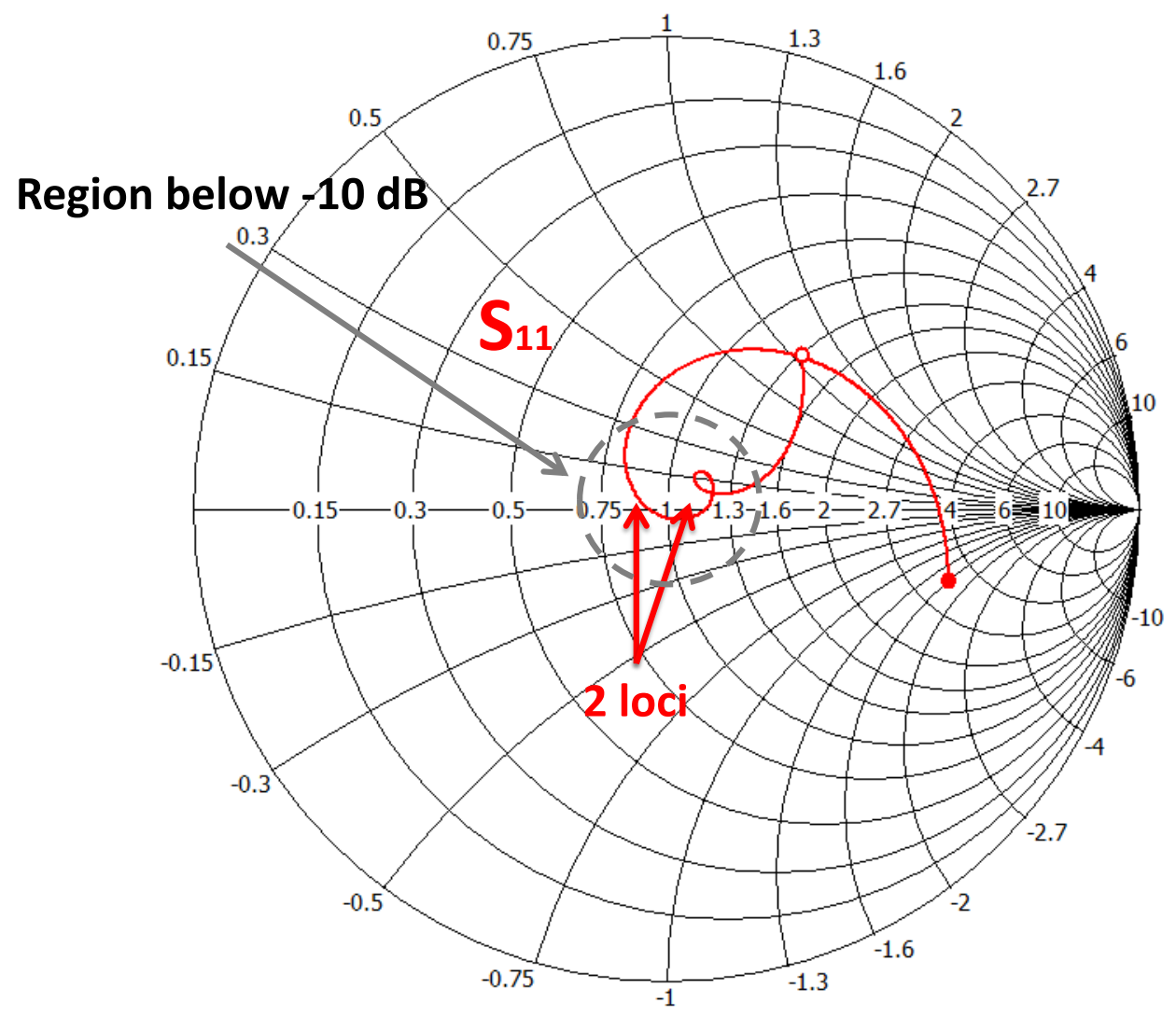

Figure 4.7: A simulation of a typical Smith chart plot with the impedance locus versus frequency of a $R S P A$.

- Size of the FR4 cavity (A and B) must be big enough, so that the FR4 is not influencing the electromagnetic waves.

Figure 4.7 displays a simulation of a typical Smith chart plot with the impedance locus versus frequency. There are two loci, one is due to the patch and the other to the slot. The size of a locus is controlled by the coupling level (with $W_{S L O T}, L_{S L O T}$ ). If $L_{T U N}$ increases, the entire locus rotates up (towards the inductive region). Accordingly, best matching, where the locus has the right size and is placed in the center of the Smith chart, can be obtained by properly adjusting stub length, the slot length and the slot width.

Fig. 4.8 shows a photography of the fabricated antenna on a PCB with its feed (on the left) and with the patch (on the right), which is still visible underneath the patch. Fig. 4.9 depicts the simulated and measured S-parameter $\left(S_{11}\right)$ of the described antenna. A TRL calibration de-embedding the connector was performed before the measurement. 


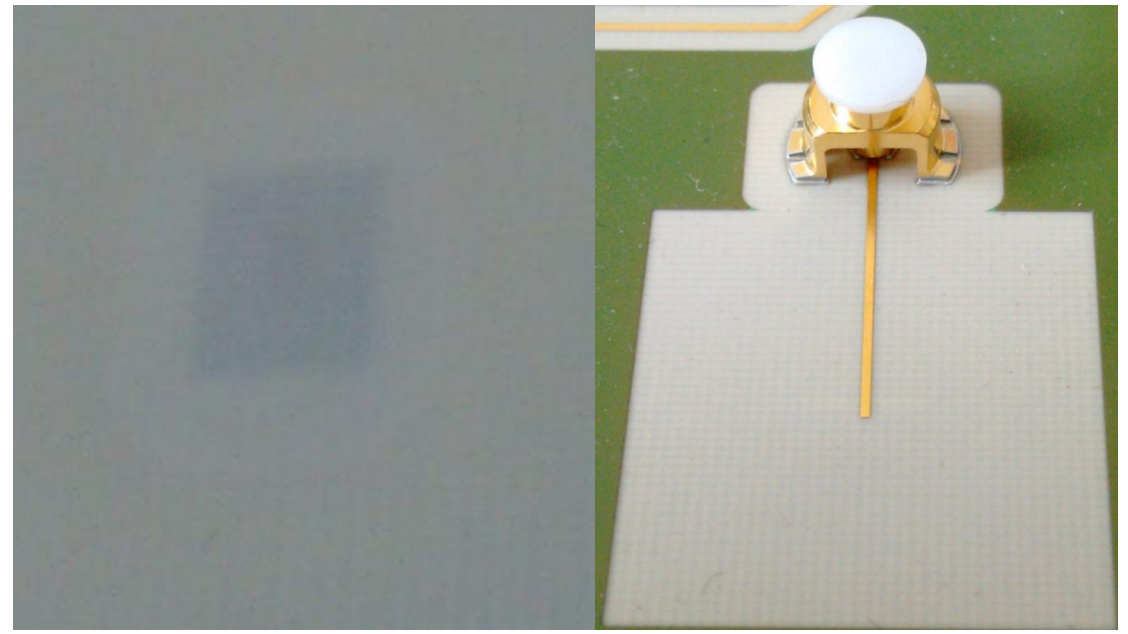

Figure 4.8: Photography of the fabricated RSPA.

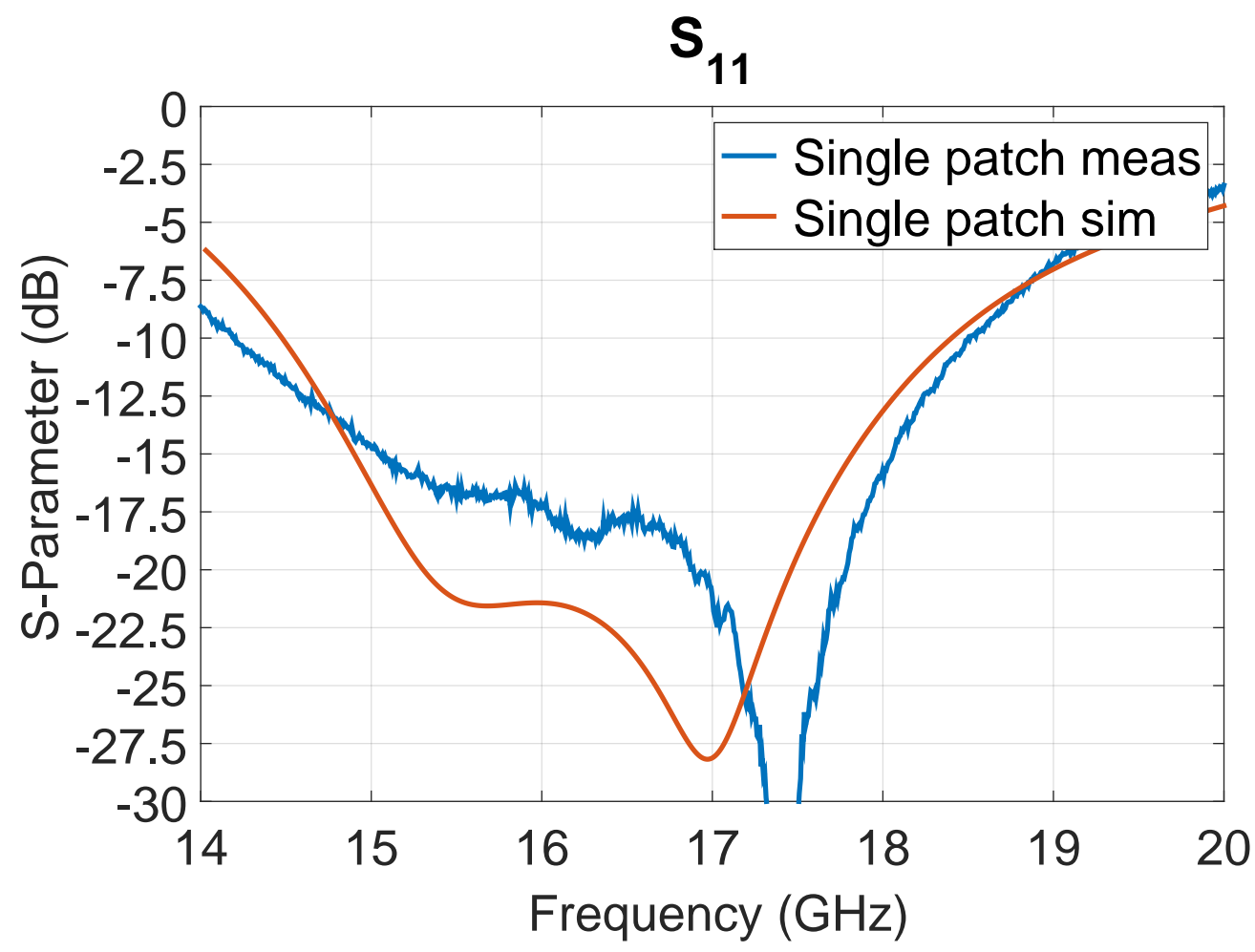

Figure 4.9: Comparison between the measured of simulated $S_{11}$ of the single patch RSPA.

The simulation and the measurement of the $S_{11}$ present very good agreement, minimal differences are due to fabrication tolerances. The reflection is less than $-10 \mathrm{~dB}$ from 15.5 to $18.5 \mathrm{GHz}$. 


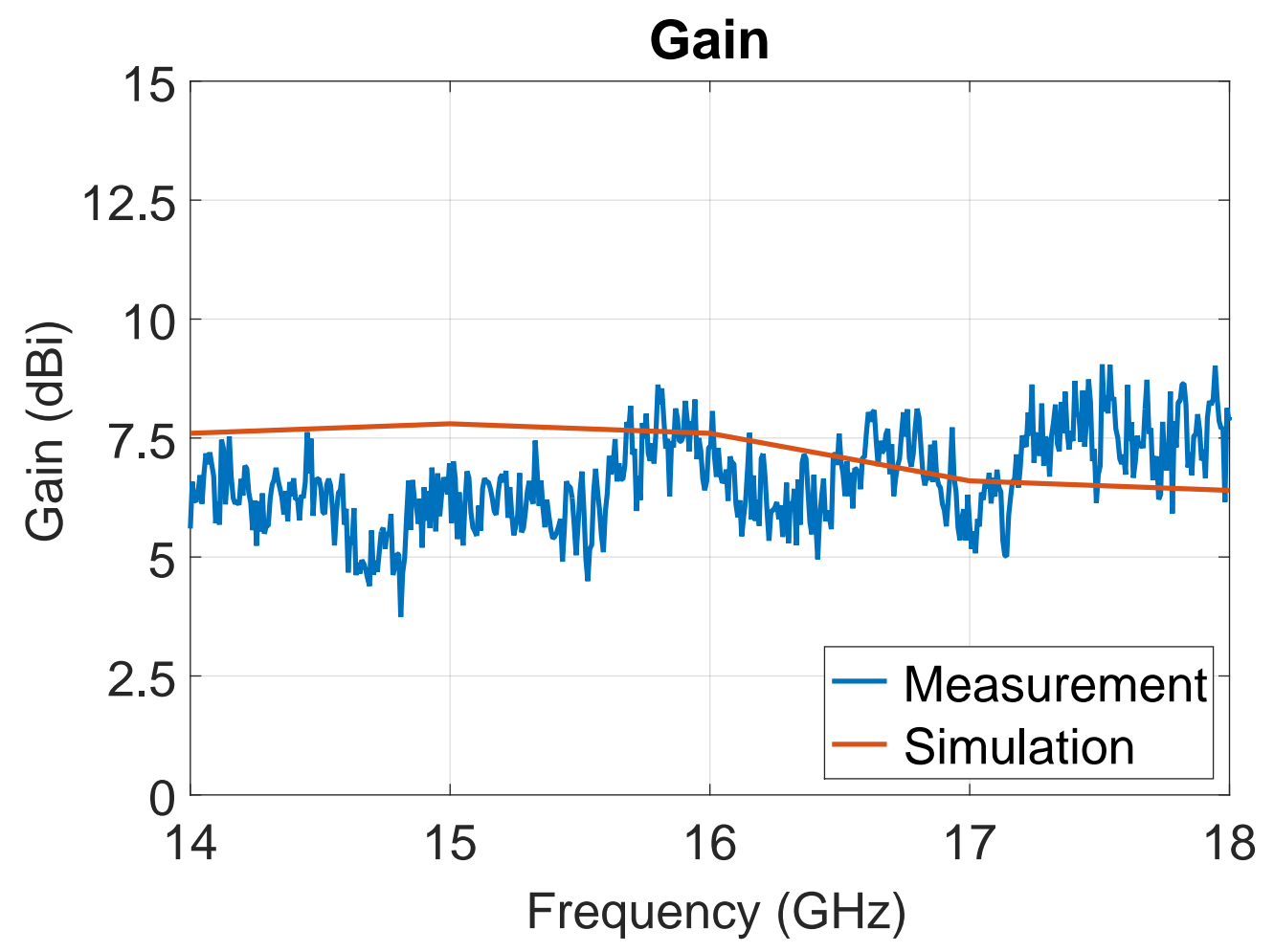

Figure 4.10: Comparison between the measured and simulated gain of the single patch RSPA.

It is important to have a good impedance matching of the antenna, but it is not sufficient. The antenna should also radiate. In order to test the antenna gain over frequency a network analyzer is used. After a calibration with a K-standard calibration kit, the port 1 is connected to a know test antenna while the port 2 is connected to the fabricated RSPA with a K to MMPX transition. Afterwards, the antennas must be placed at a certain distance R. The antennas can be aligned by searching the maximum $S_{21}$ level on the network analyzer.

$$
G_{R S P A}=S_{21}-G_{T E S T}-20 \log _{10}\left(\frac{\lambda}{4 \pi R}\right)^{2}
$$

Using the equation (4.5) and knowing the gain of the test antenna $G_{T E S T}$, the distance between antennas $\mathrm{R}$, and the measured $S_{21}$ the gain of the antenna can be calculated.

Fig. 4.10 shows the calculated gain. The gain is about $7 \mathrm{dBi}$ over the whole frequency range. The measurement fits rather well the simulation, specially considering that it was not performed in an anechoic chamber. The designed antenna is operating properly. Therefore, the accumulated experience can be used for designing the RX (an array consisting of a 4 patch RSPA antennas) and the TX (an array consisting of a 2 patch RSPA antennas) 
antennas.

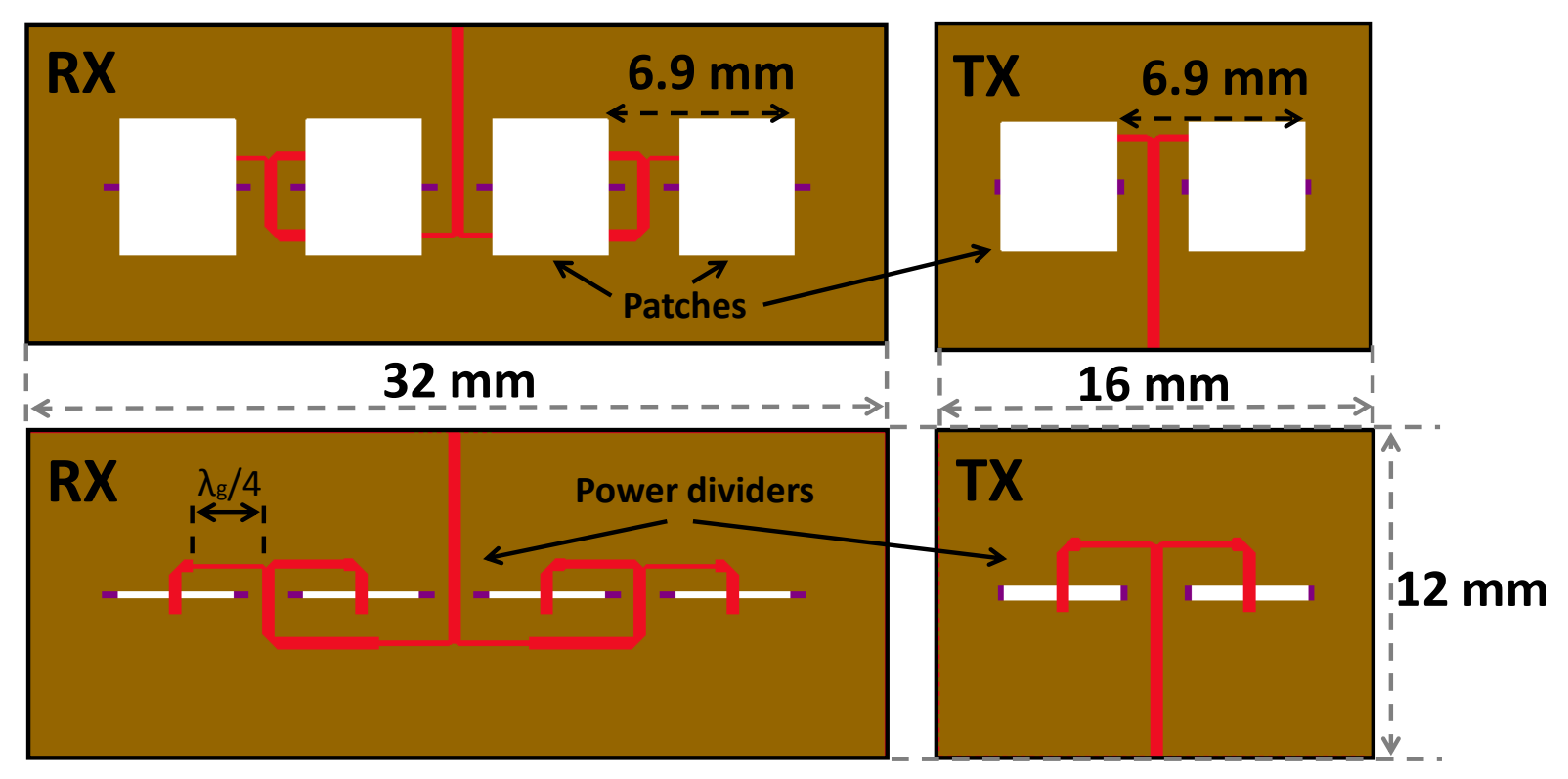

Figure 4.11: $T X$ and $R X$ layout.

\section{RX and TX arrays}

In this section the TX and RX antennas are introduced. They consist of 2 RSPA and 4 RSPA arrays, respectively. The available surface for the TX antennas is $16 \mathrm{~mm} \times 12 \mathrm{~mm}$ and for the RX antenna $32 \mathrm{~mm} \times 12 \mathrm{~mm}$. Fig. 4.11 depicts the layout of the RX and TX arrays. The patch antennas (layer 5) are represented in white color, the ground (layer 2) with the resonant slots in brown and the microstrip feed network in form of $\mathrm{T}$ junctions (layer 1) in red. Layers 3 and 4 are reserved for DC routing purposes and empty within the RF antennas's surface. Due to this constrain the distance between array elements is smaller than $\lambda_{0} / 2=9.23 \mathrm{~mm}$ and equal to $d=6.9 \mathrm{~mm}$. As a consequence, the number of sidelobes is reduced and the main lobe width is increased. In order to feed the arrays a $\lambda_{g} / 4$ power splitter network is designed (see in red on Fig. 4.11), since a Wilkinson power divisor does not fit in the available area. It must be noted that the distance between antenna elements is related to the free-space $\lambda_{0}=\frac{c_{0}}{f_{0}}=18.46 \mathrm{~mm}$ while the length of the microstrip line stages of the $\mathrm{T}$ junction is $\frac{\lambda_{g}}{4}=\frac{c_{0}}{f_{0} \sqrt{\epsilon_{e f f}}}=2.77 \mathrm{~mm}$, considering $f_{0}=16.25 \mathrm{GHz}, \epsilon_{e f f}=2.77$ (from [7]), $c_{0} \approx 3 \cdot 10^{8} \mathrm{~m} / \mathrm{s}$. 

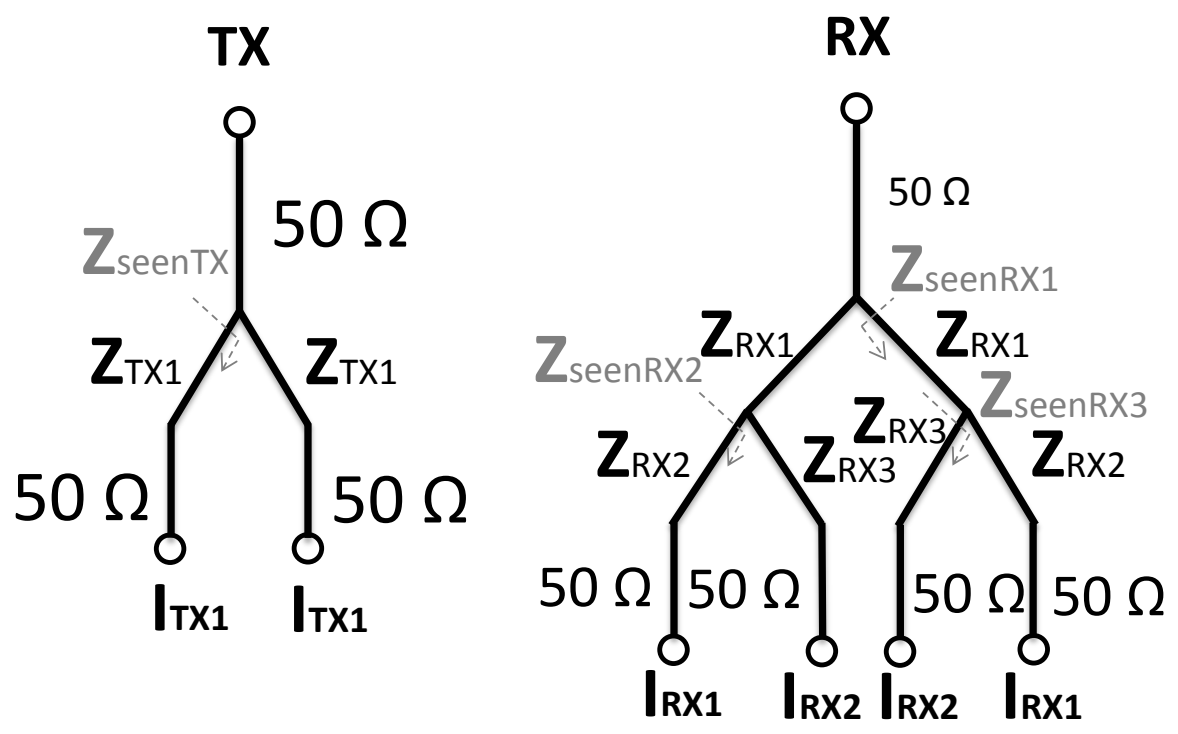

Figure 4.12: A Sketch of the RX and TX feed networks.

Fig 4.12 depicts a sketch of the desired feed networks. In order to calculate the line impedance of the TX feed network $Z_{T X 1}$ two constrains must be consireded. First, the amplitude of the provided current must be equal in the output ports. Second, the output ports must be matched to an impedance of $Z=50 \Omega$. Considering that, the value of $Z_{T X 1}$ can be calculated straightforward using the quarter-wave impedance transformer equation as equations (4.6) and 4.7) show.

$$
\begin{gathered}
Z_{\text {seenTX }}=100=\frac{\left(Z_{T X 1}\right)^{2}}{50} \\
Z_{T X 1}=50 \sqrt{2} \Omega
\end{gathered}
$$

In order to reduce the side-lobes of the RX antenna pattern a tapering of the amplitude of RX array element's is performed. Fig. 4.13 illustrates the weights of the current amplitude of the antenna RX $W_{R X 1}=0.59$ and $W_{R X 2}=0.95$. In this case, unequal current must be distributed between the 4 ports. As a consequence, using classical circuit theory, the two following conditions must be fulfilled:

$$
\begin{aligned}
Z_{\text {seen } R X 2} / / Z_{\text {seen } R X 3}=\frac{Z_{R X 2}^{2}}{50} / / \frac{Z_{R X 3}^{2}}{50} & =50 \\
\frac{Z_{R X 2}^{2} Z_{R X 3}^{2}}{50\left(Z_{R X 2}^{2}+Z_{R X 3}^{2}\right)} & =50
\end{aligned}
$$




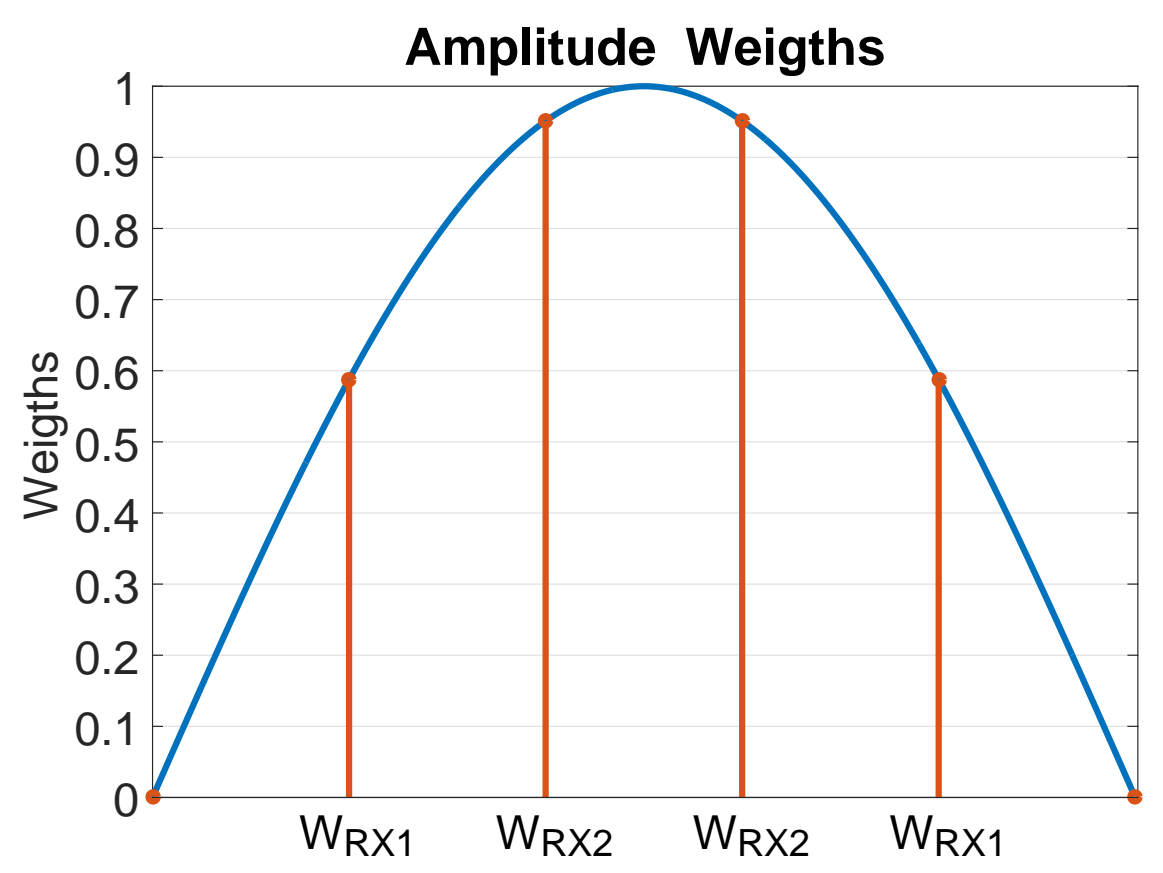

Figure 4.13: Weights of amplitude of the RX antenna elements

$$
\begin{array}{r}
Z_{\text {seen } R X 2} I_{R X 1}=Z_{\text {seen } R X 3} I_{R X 2} \\
\frac{Z_{R X 2}^{2}}{50} I_{R X 1}=\frac{Z_{R X 3}^{2}}{50} I_{R X 2} \\
\frac{Z_{R X 2}}{Z_{R X 3}}=\sqrt{\frac{I_{R X 2}}{I_{R X 1}}} \\
\frac{Z_{R X 2}}{Z_{R X 3}}=\sqrt{\frac{W_{R X 2}}{W_{R X 1}}}=\sqrt{\frac{0.95}{0.59}}=1.27
\end{array}
$$

In order to calculate $Z_{R X 1}$ the same procedure to $Z_{T X 1}$ must be followed. Table 4.1 shows a summary of the calculated impedances and its corresponding microstrip line widths. They have been calculated using (4.7), 4.8), 4.9) and the classical wheeler equation [8].

Fig. 4.14 depicts a graphic capture of the 3D model of the TX (bottom) and RX (top) feeds while Fig. 4.15 presents the simulation of its transmission S-parameters. The TX curves (TX- $S_{12}$ and TX- $S_{13}$ ) overlap, considering that the circuit is symmetric. They have a value of $-3.26 \mathrm{~dB}$ at $16.25 \mathrm{GHz}$. This value results appropriate, since the half of the power must be split equally among both ports and some extra losses due to propagation must be added. However, the values of RX-S $S_{12}$ and RX- $S_{13}$ are not equal, because the feed is designed to have a cosine variation in amplitude, as Fig. 4.13 illustrates. The value of 


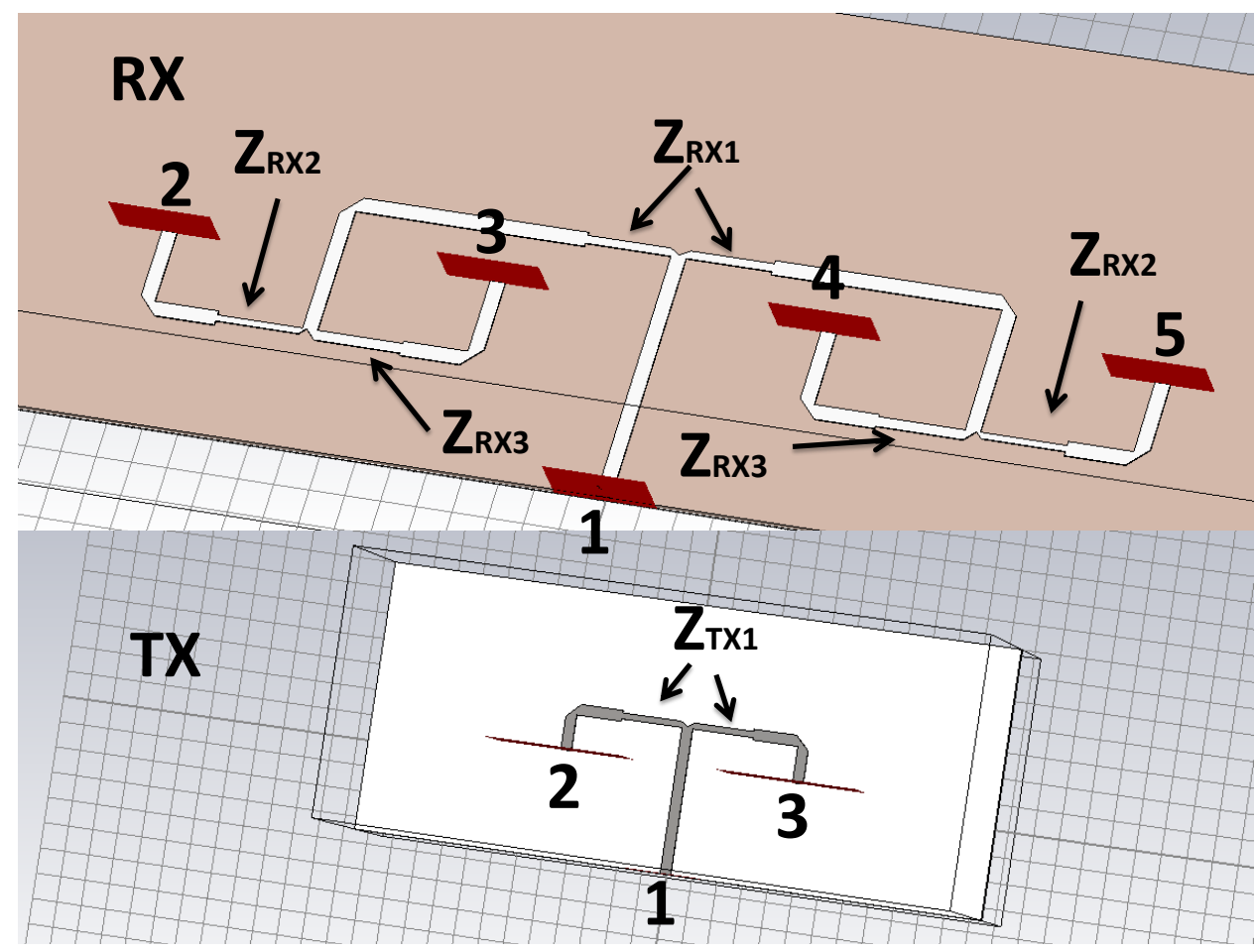

Figure 4.14: Illustration of the simulated $R X$ (bottom) and $T X$ (top) feeds in a commercial software.

\begin{tabular}{ccc} 
Index & $\mathrm{Z}(\Omega)$ & $\mathrm{w}(\mathrm{mm})$ \\
\hline TX1 & 70.71 & 0.238 \\
RX1 & 70.71 & 0.238 \\
RX2 & 80.82 & 0.180 \\
RX3 & 63.64 & 0.292
\end{tabular}

Table 4.1: Feed network impedances and its corresponding micorstrip line widths.

RX- $S_{12}$ and RX-S $S_{13}$ at $16.25 \mathrm{GHz}$ are $-7.4 \mathrm{~dB}-5.5 \mathrm{~dB}$, respectively.

$$
\begin{aligned}
\frac{Z_{R X 2}}{Z_{R X 3}}=\sqrt{\frac{W_{R X 2}}{W_{R X 1}}} & =\sqrt{\frac{0.95}{0.59}}=1.27 \\
\frac{Z_{R X 2}}{Z_{R X 3}} & \approx \frac{10^{\frac{-S_{13}}{20}}}{10^{\frac{-S_{12}}{20}}}=1.25
\end{aligned}
$$

Equation $(4.10)$ is used in order to compare the simulated and the calculated restult. The ratio in linear magnitude of $\frac{S_{13}}{S_{12}}$ must be equal to the previously calculated with $\frac{Z_{R X 2}}{Z_{R \times 3}}$. In this case there is a small 0.02 error due to the fact that the theoretical equations for calculating impedances of a micrsotrip lines and $\epsilon_{e f f}$ and the software used slightly different 


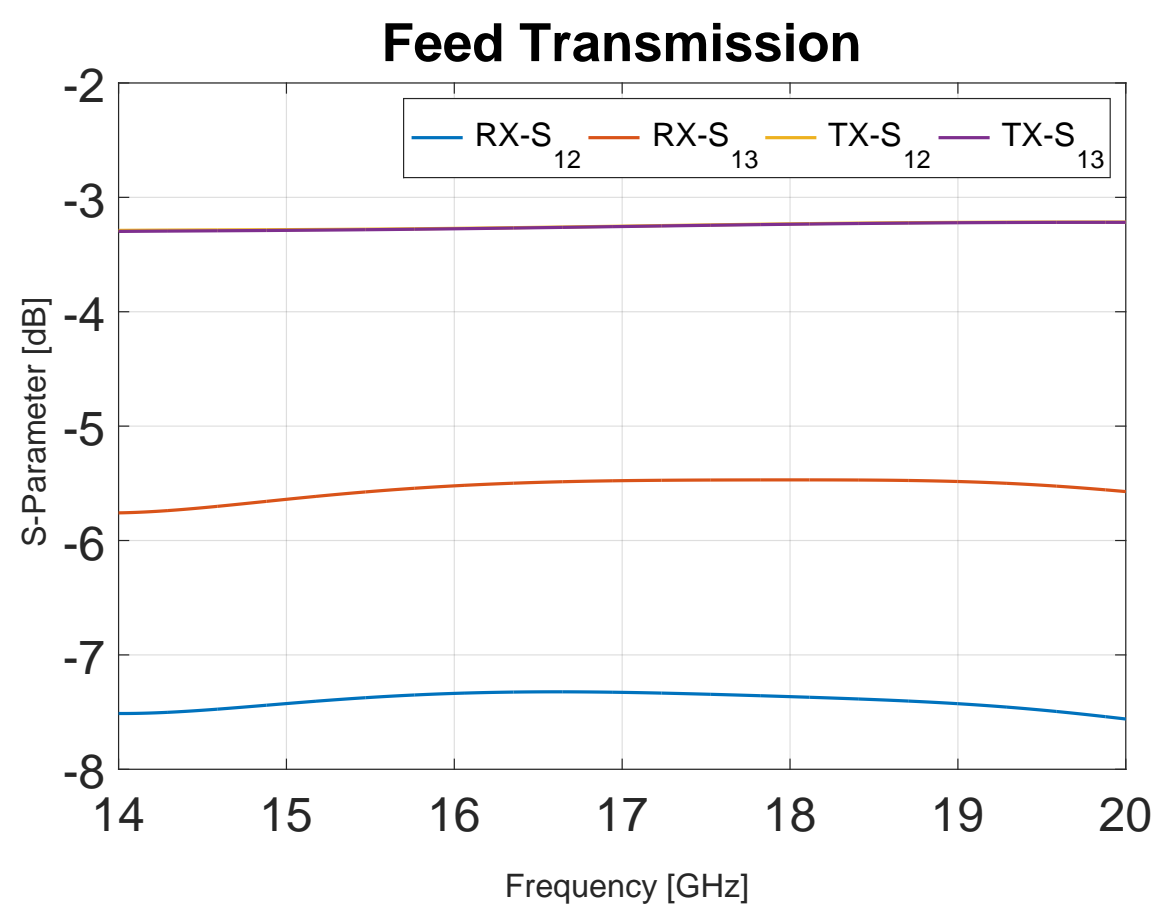

Figure 4.15: Feed of the RSPA TX and RX antenna arrays.

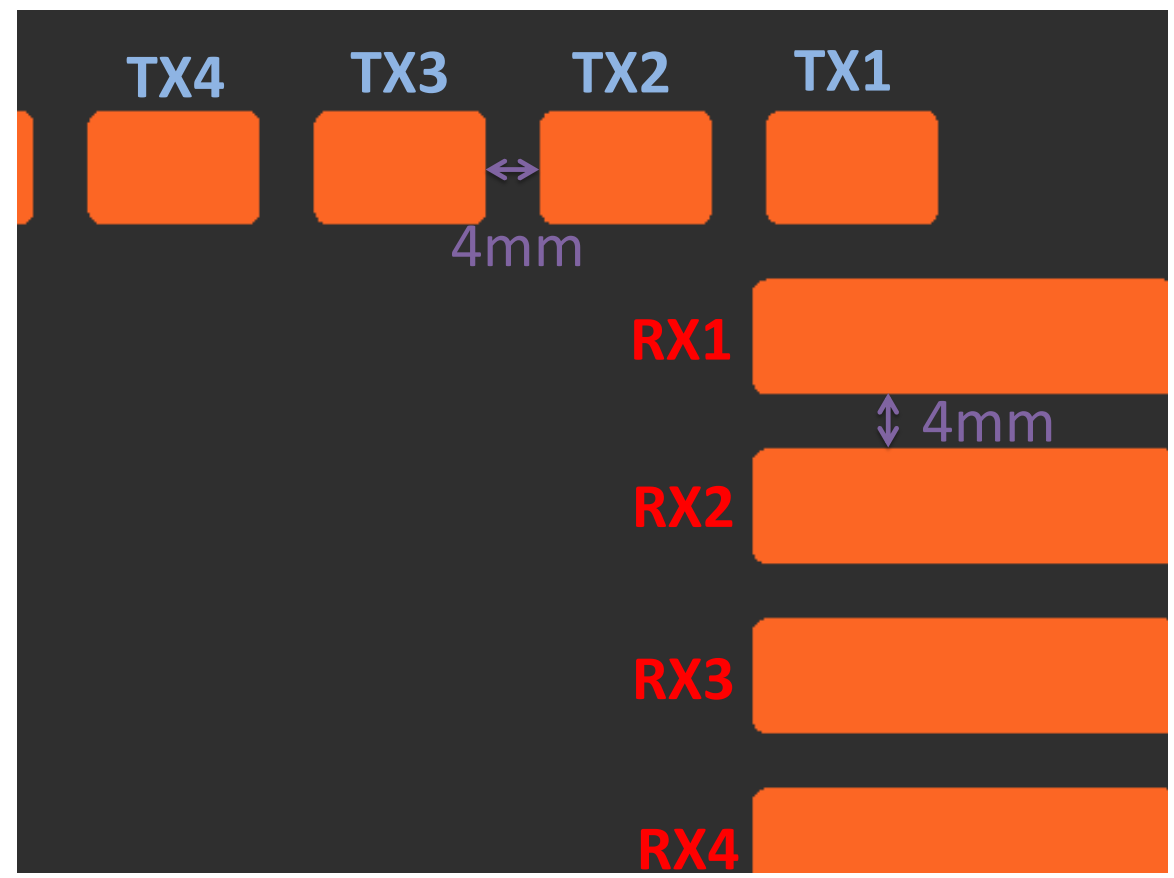

Figure 4.16: Gerber file of the milled cavities in the PCB in orange. 
boundary conditions. The results are good enough to follow the next step in the design process.

Once the feed's design and the single element's design are finished, the design of the whole antenna array should be straightforward. Unfortunately, a difficulty which adds an extra boundary condition force to redesign of the antenna parameters for the RSPA TX and RX arrays. As previously mentioned, the FR4 layers are milled, in such a manner that between two milled cavities a bridge is remaining. This bridge cannot be smaller than $4 \mathrm{~mm}$ (see Fig. 4.16). In order to fulfill this specification a redesign of the antenna parameters is required.

Table 4.2 describes the main parameters of the fabricated RX and TX antenna array.

\begin{tabular}{ccc} 
Parameter $(\mathrm{mm})$ & $\mathrm{TX}$ & $\mathrm{RX}$ \\
\hline$L_{P A T C H}$ & 4.7 & 5 \\
$W_{P A T C H}$ & 4.25 & 4.25 \\
$L_{S L O T}$ & 4.8 & 5.5 \\
$W_{S L O T}$ & 0.6 & 0.3 \\
$\mathrm{~A}$ & 8 & 8 \\
$\mathrm{~B}$ & 12 & 30 \\
$L_{T U N}$ & 0.7 & 0.7
\end{tabular}

Table 4.2: New antenna parameters of the RX and TX antennas. Fig. 4.6 illustrates an image with a description of the parameters.

Fig. 4.17 depicts a comparison between the simulated and measured input reflection of the fabricated RX and TX antennas. The measurement is performed with a TRL calibration, in such a manner that the connector is de-embedded. The measured reflection is less than $-10 \mathrm{~dB}$ over the targeted frequency range (from 15 to $17.5 \mathrm{GHz}$ ). The differences between measurement and simulation are mainly due to the influence of the FR4 substrates on the redesigns. With the redesign a last minute change was mandatory. In it the air cavities diminished in such a manner that the influence of the FR4 layers is not anymore negligible. The FR4 is a very inexpensive substrate and the fabrication tolerances are quite high. The used values of the FR4 of $\epsilon_{r}$ and $\tan (\delta)$ are 4.3 and 0.025 , respectively.

Fig. 4.18 depicts a comparison between the simulated and measured gain of the fabricated RX and TX antennas. This measurement is performed with a network analyzer and a test antenna settled at a certain distance R. Using the equation (4.5) and knowing the gain of the test antenna $G_{T E S T}$, the distance between antennas $\mathrm{R}$ and the measured $S_{21}$ the gain of the antenna can be calculated. The measured gains are approximately $G_{T X}=6.5$ and $G_{R X}=8.5$ over the targeted frequency range (from $15 \mathrm{GHz}$ to $17.5 \mathrm{GHz}$ ). The differences of between measurement and simulation are mainly due to the influence of 


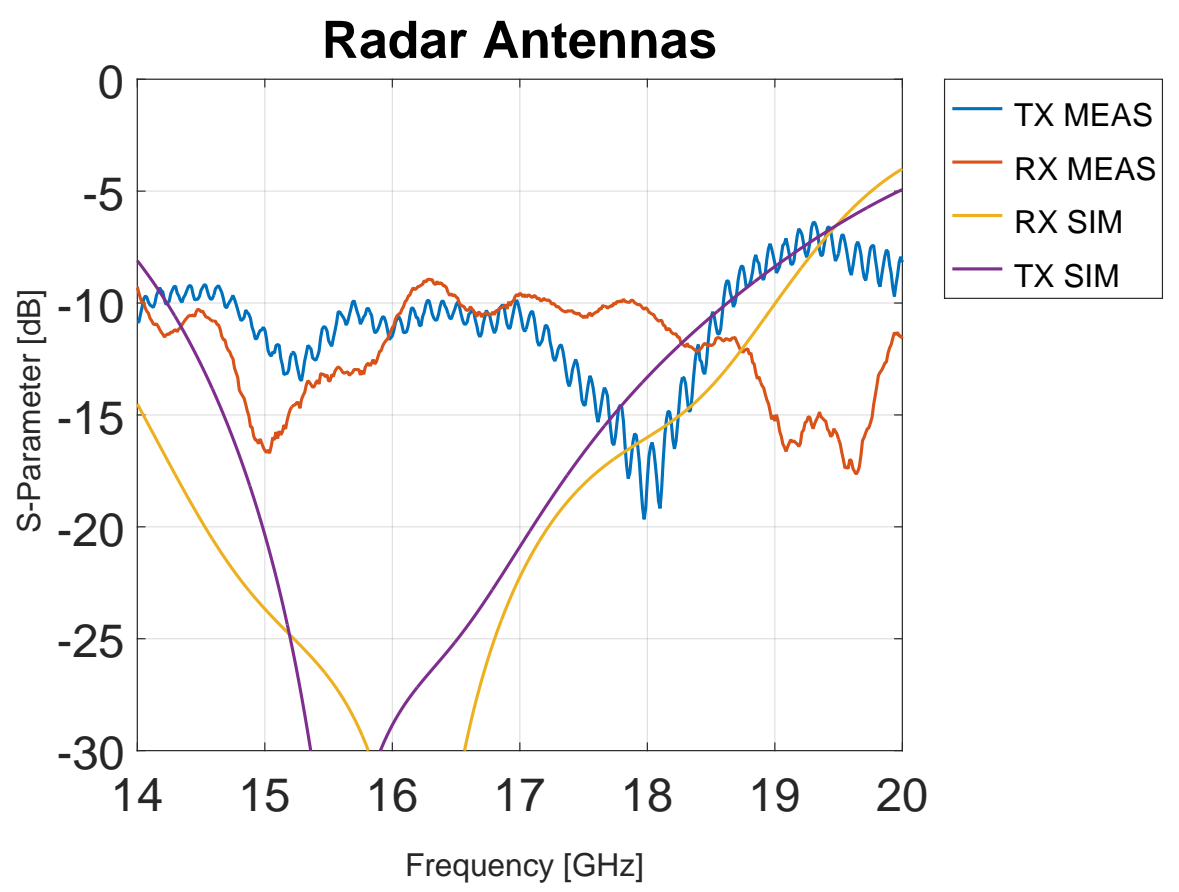

Figure 4.17: Comparison between the simulated and measured matching of the $R X$ and TX RSPA.

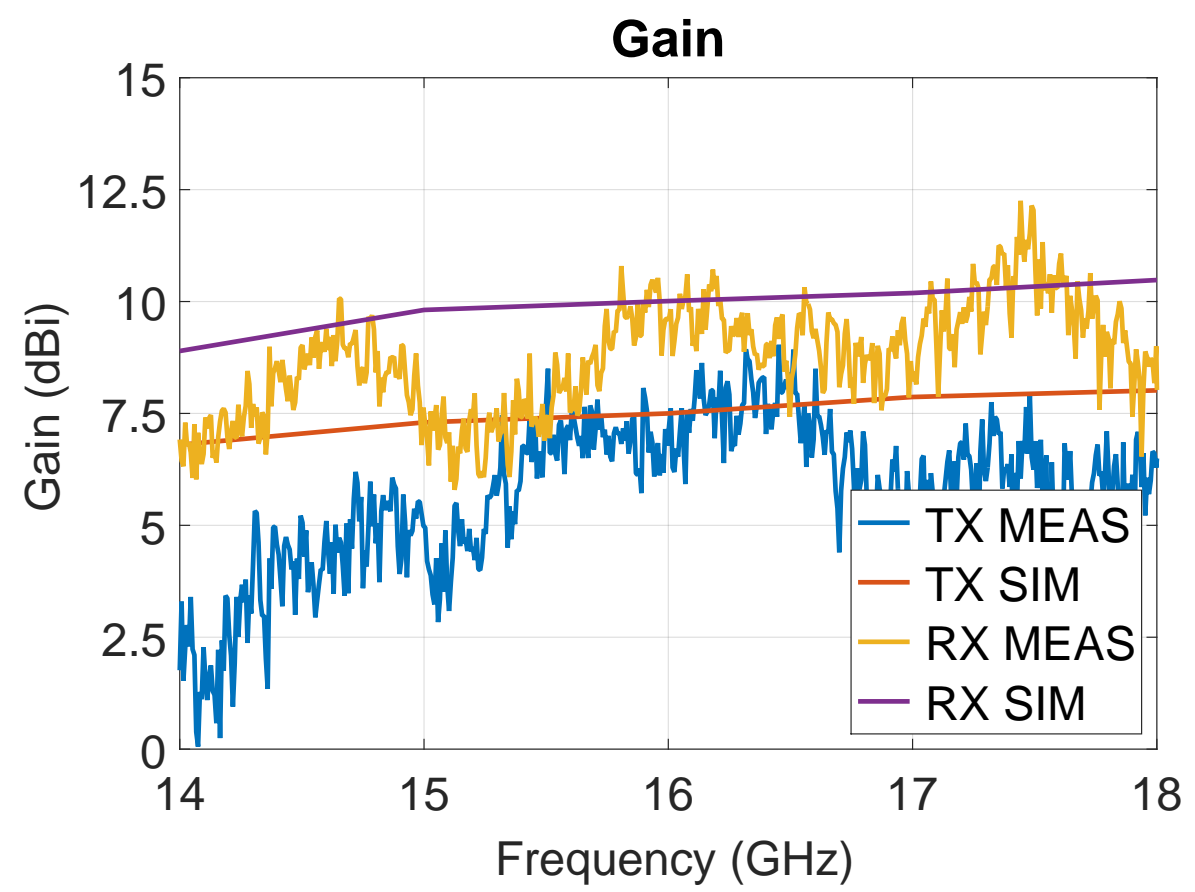

Figure 4.18: Comparison between the simulated and measured gain of the $R X$ and TX RSPA. 
the FR4 substrates on the redesigns.

As a summary, the last minute redesign worsened the antennas performance in comparison with the first single patch. However, the antenna performance in terms of gain and return loss is good enough to be integrated into a radar frontend.

\subsubsection{Vertical Transition}

The main purpose of this chapter is to present a reduced version of a RF fronted of a MIMO radar. The proposed layer stack-up has already been defined (see Fig.4.6). A vertical transition through FR4 is very advantageous in order to efficiently utilize the PCB surface, since it multiplies per two the available area for a single RF circuit. Several microstrip line vertical transitions have been reported in the scientific literature [9] [10] [12] [13] [14].

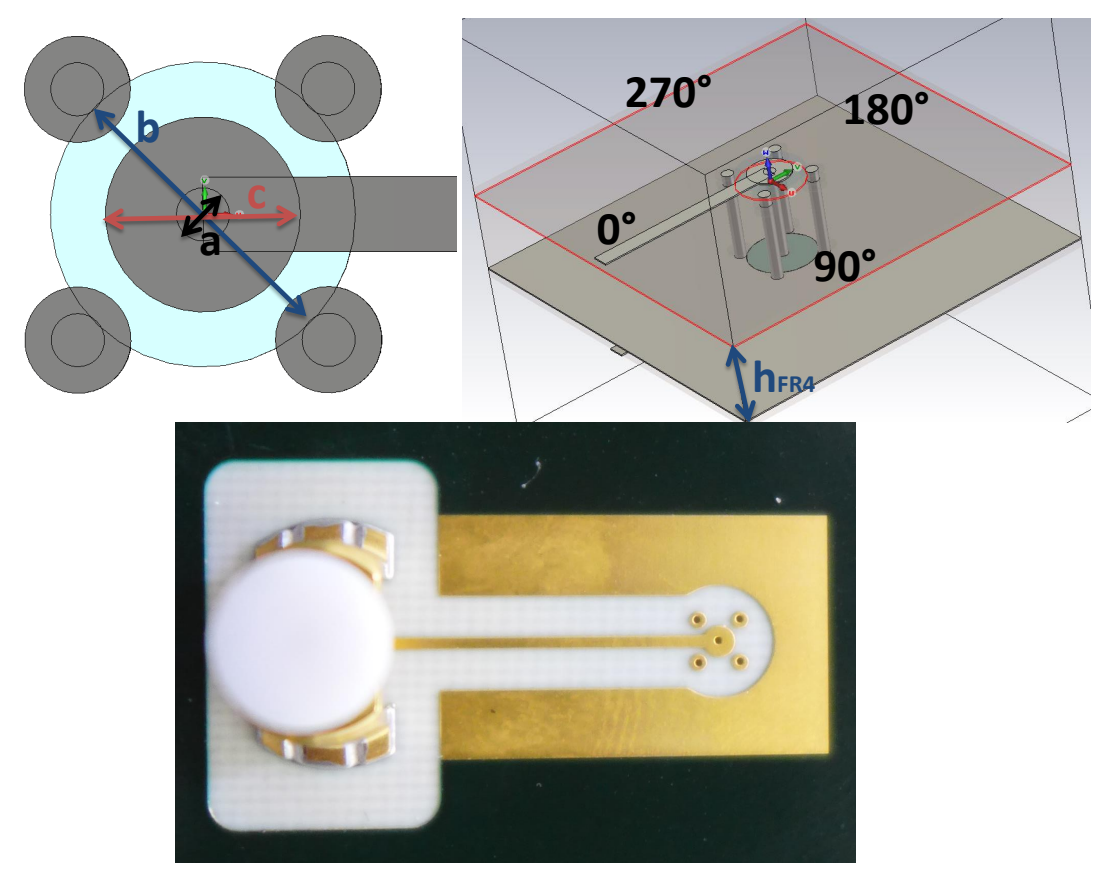

Figure 4.19: 3D model of the fabricated vertical transition (top). Photography of the fabricated device (bottom).

In this work, a coaxial vertical transition is designed as Fig. 4.19 shows. It has a central via, which act as an inner conductor and four side vias, which act as a outer conductor and are connected to ground. The number of outer vias is set to four, in such a manner $90^{\circ}$, $180^{\circ}$ and $270^{\circ}$ vertical transitions are suitable (see Fig. 4.19 top-right). Furthermore, the microstrip line ends on a circular metallic surface of diameter c in order to compensate the inductive effects of the vias and to provide a more broadband transition. The diameter of all vias is $a$, the distance among the outer vias is $b$ while $c$ is the disk's diameter in which 
the microstrip line finishes as Fig. 4.19 top-left illustrates.

$$
Z=\frac{138}{\sqrt{\epsilon_{r}}} \log _{10}\left(\frac{b}{a}\right)
$$

Firstly, $a$ was selected to be $0.30 \mathrm{~mm}$, a common value for PCB design. After that, a first approximation of $b$ was calculated forcing the equation (4.11) to be $50 \Omega$ with $1.45 \mathrm{~mm}$ as a result. Later, a parametric analysis varying $c$ shows that $c=1.10 \mathrm{~mm}$ improves the transmission. At the end, a fine tune of $b$ is performed obtaining $b=2.00 \mathrm{~mm}$ as a result.

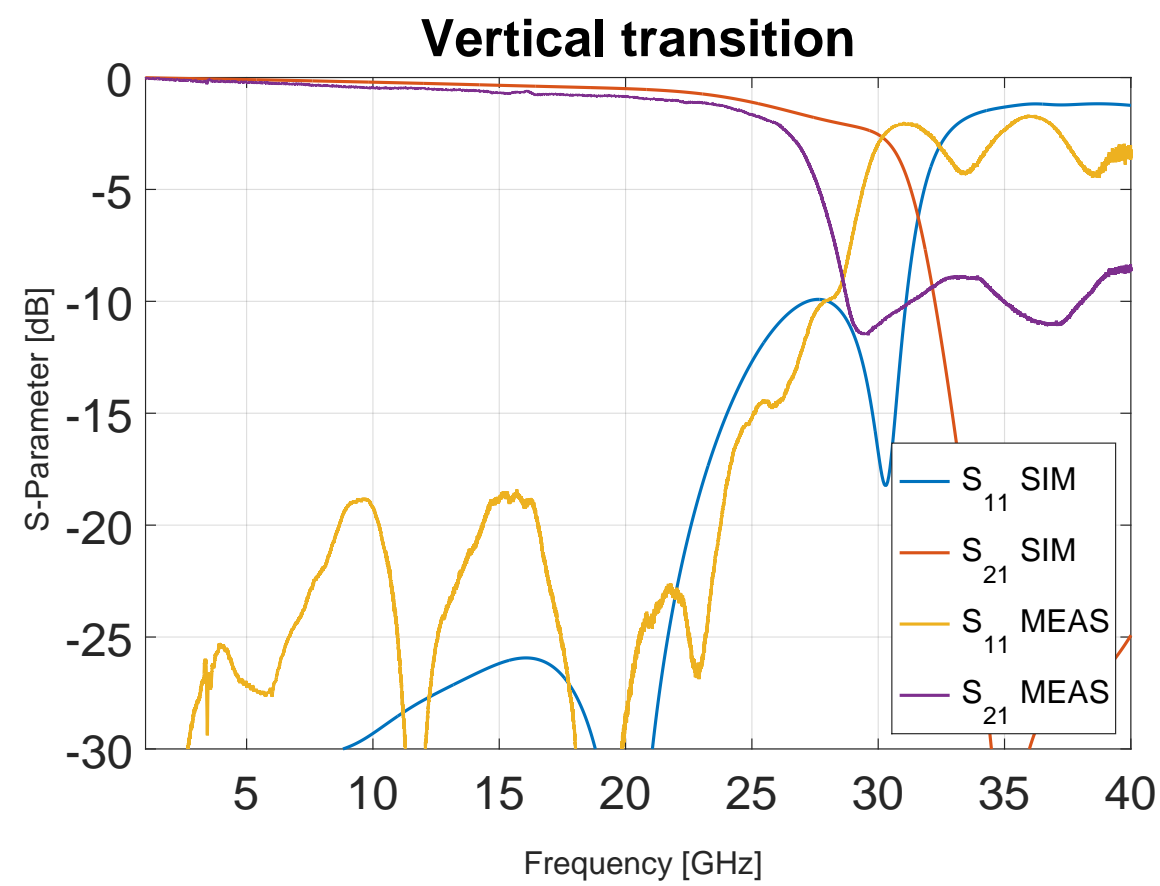

Figure 4.20: Comparison between simulated and measured S-parameter of the fabricated device.

Fig. 4.20 illustrates a comparison between the simulated and measured S-parameters of the vertical transition. The $S_{21}$ is approximately $0.6 \mathrm{~dB}$ and the $S_{11}$ is below $-18 \mathrm{~dB}$ over the targeted frequency range (from $15 \mathrm{GHz}$ to $17.5 \mathrm{GHz}$ ). The measurements fit rather well the simulation over the targeted frequency range. The differences between measurement and simulation are mainly due to the influence of fabrication tolerances, especially of the FR4 substrates.

As a summary, the fabricated vertical transition is very good in terms of matching and transmission and can be integrated into a radar frontend. 


\subsubsection{Three-Way Planar Wilkinson Power Divider}

E. J. Wilkinson introduced a power divider which improves the output reflection and the isolation of a T-junction [7] by adding a resistor among output ports. If more than 2 ports are needed, there are two possible configurations as Fig. 4.21 illustrates.

\section{DELTA CONFIGURATION STAR CONFIGURATION}
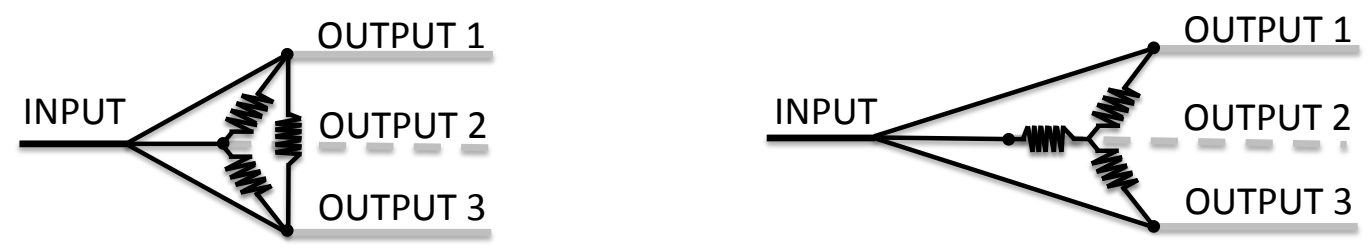

Figure 4.21: 3-way Wilkinson divider. Delta configuration (left) and star configuration (right).

To implement this circuit in a planar based transmission line as the microstrip line is not convenient, thus it has axial symmetry instead of planar symmetry. However, a way to approach this cumbersome problem, which reduces the number of resistances has been presented [16]. This power divider is not perfect, since it is missing an isolation resistor between two output ports. Nevertheless it is very easy to implement in conventional PCB technology and presents still some advantages is compared to a T-junction.

Fig. 4.22 illustrates a photography (left) and the layout (right) of the fabricated device.

Fig. 4.23 displays a comparison between the simulated and measured input reflection $\left(S_{11}\right)$ and transmission $\left(S_{12}, S_{13}\right.$ and $\left.S_{14}\right)$ S-parameters of the fabricated 3 way planar Willkinson divider. The simulation fits rather well the measurement. The measured and simulated input reflection $S_{11}$ are bellow $-20 \mathrm{~dB}$ from $15 \mathrm{GHz}$ to $17.5 \mathrm{GHz}$. The transmision S-parameters $\left(S_{12}, S_{13}\right.$ and $\left.S_{14}\right)$ are all around $-5 \mathrm{~dB}$. This results appropiate considering that the power divider is designed to provide with a $1 / 3$ of the input power to each of its output ports (see equation (4.12) ) and that extra propagration losses are also included.

$$
\begin{aligned}
& P_{\text {out }}=\frac{P_{\text {in }}}{3} \\
& S_{\text {1out }}=\frac{\sqrt{P_{\text {out }}}}{\sqrt{P_{\text {in }}}}=\sqrt{\frac{1}{3}} \\
& S_{\text {lout }}(d B)=20 \log _{10}\left(\sqrt{\frac{1}{3}}\right) \approx-4.8
\end{aligned}
$$




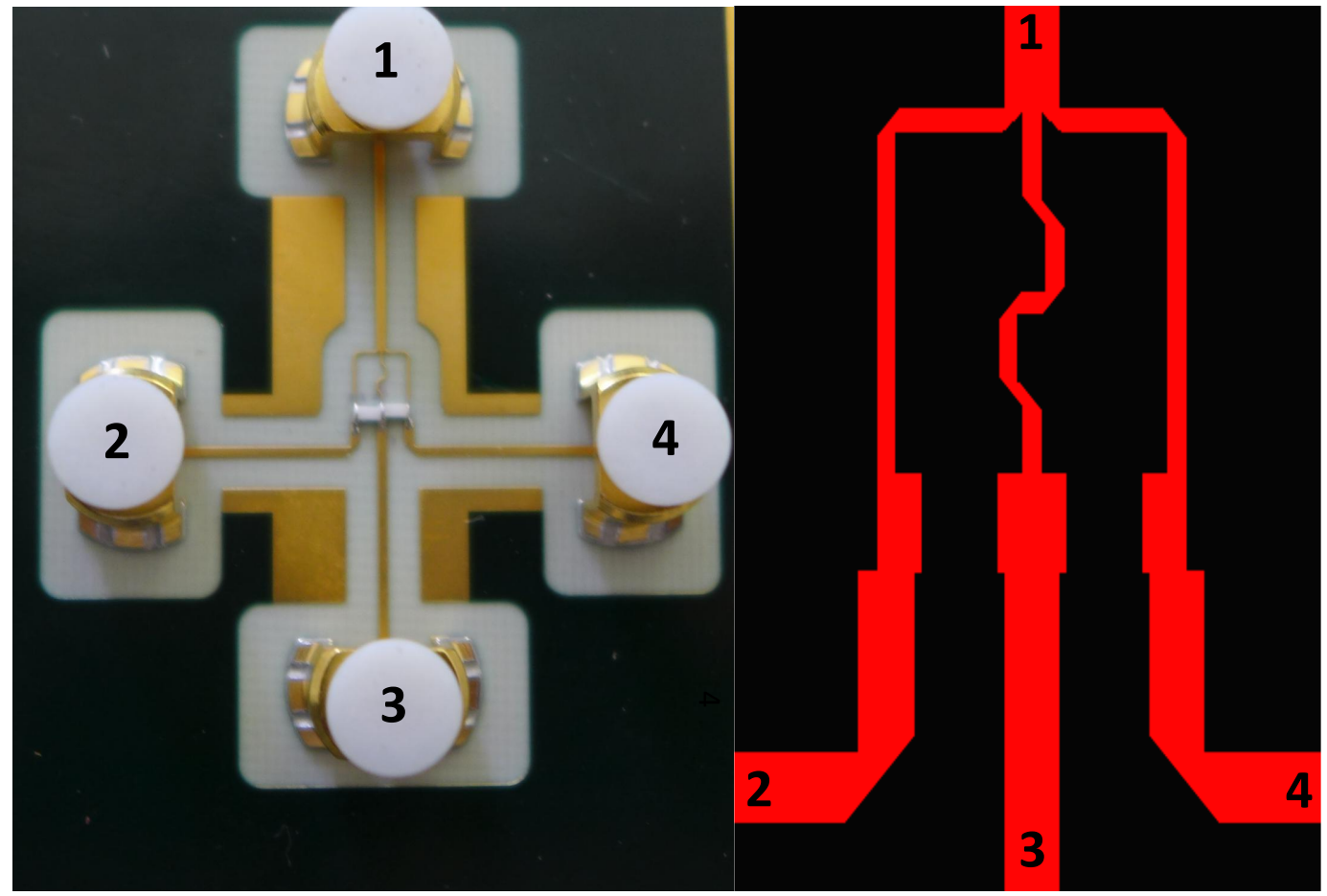

Figure 4.22: Image of the fabricated 3-way Wilkinson divider. Photography (left) and layout (right).

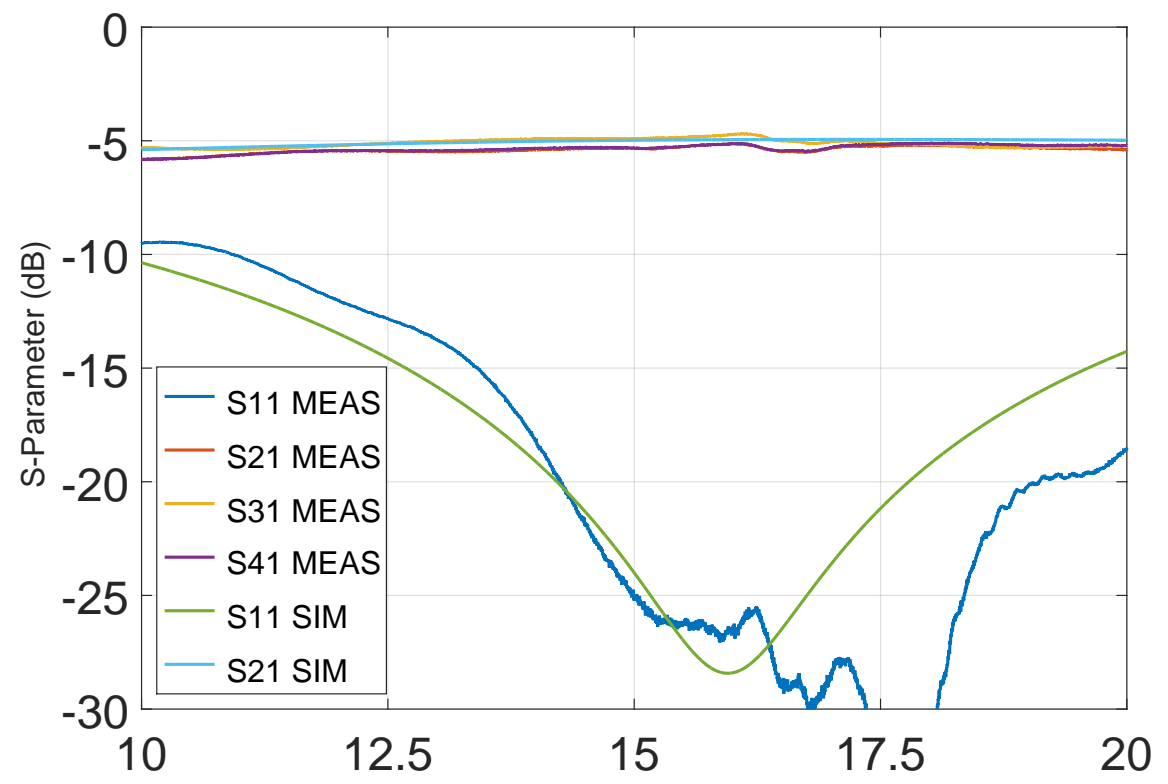

Figure 4.23: Comparison between simulated and measured input reflection $\left(S_{11}\right)$ and transmission $\left(S_{12}, S_{13}\right.$ and $\left.S_{14}\right)$ S-parameters. 
Fig. 4.24 shows a comparison between the simulated and measured output reflection $\left(S_{22}, S_{33}\right.$ and $\left.S_{44}\right)$. The simulation of $S_{44}$ is not displayed because it would be overlapped by the simulated $S_{22}$. The differences between simulated and measured values are due to the parasitic effects of the resistors, which are not considered in the simulation. The measured output reflections are below $-12 \mathrm{~dB}$ over the frequency of operation (from 15 to $17.5 \mathrm{GHz})$.

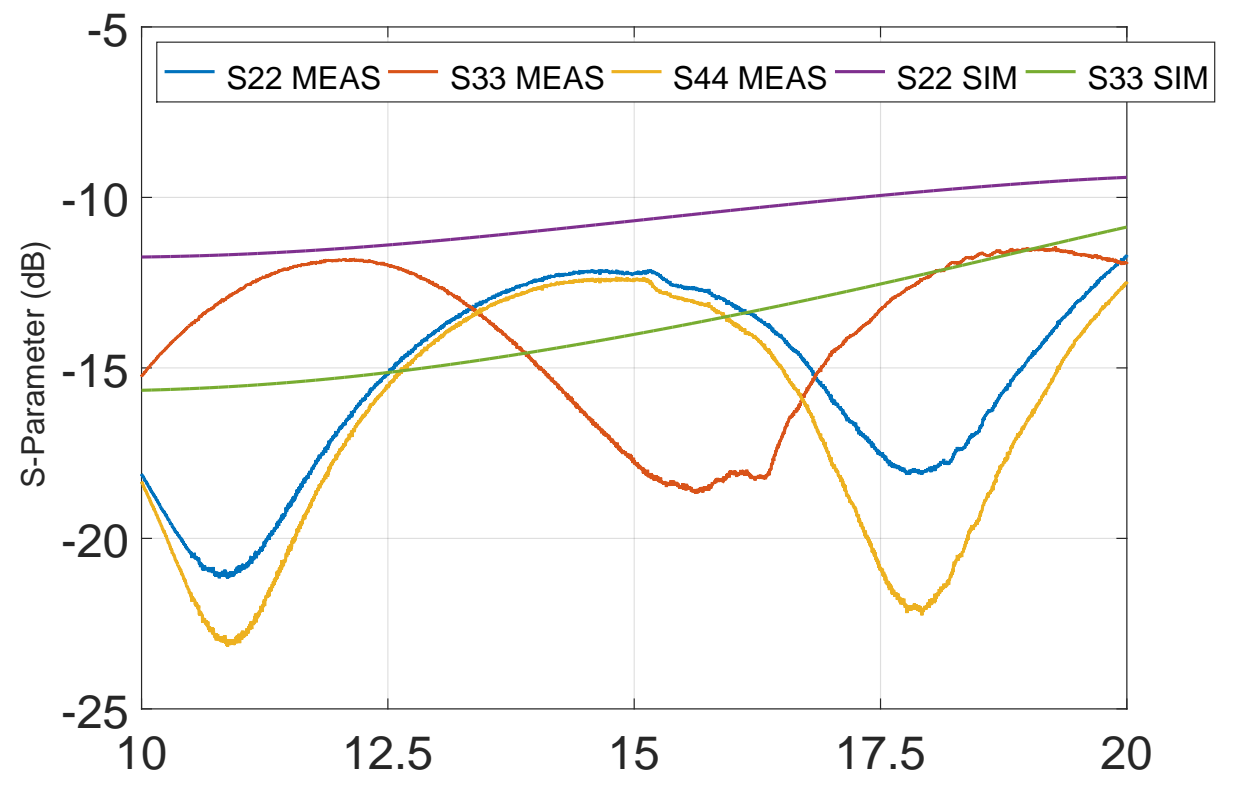

Figure 4.24: Comparison between simulated and measured output reflection $\left(S_{22}, S_{33}\right.$ and $\left.S_{44}\right)$ S-parameters.

Fig. 4.25 depicts a comparison between simulated and measured isolation $\left(S_{23}, S_{34}\right.$ and $\left.S_{43}\right)$ S-parameters. The simulation of $S_{23}$ is not displayed because it would be overlapped by the simulated $S_{43}$. The differences between simulated and measured values are due to the parasitic effects of the resistors, which are not considered in the simulation. It can be seen, that in the frequency of operation (from 15 to $17.5 \mathrm{GHz}$ ), the paths that are connected with a resistor (from port 2 to port 3 and from port 4 to port 3 ) present higher isolation that the one that is not connected with the resistor (from port 2 to port 4 ).

In this section, the main building blocks of the radar system and its measurements have been introduced. These structures provide the backbone of the radar presented in this chapter and are a very important part of other radar systems in developed AGI. The gathered information has been used to create a power level based system designs and front-end level simulations, which are essential for the correct front-end design. Some of the required components as switches or amplifiers have been presented in chapter 3 . 


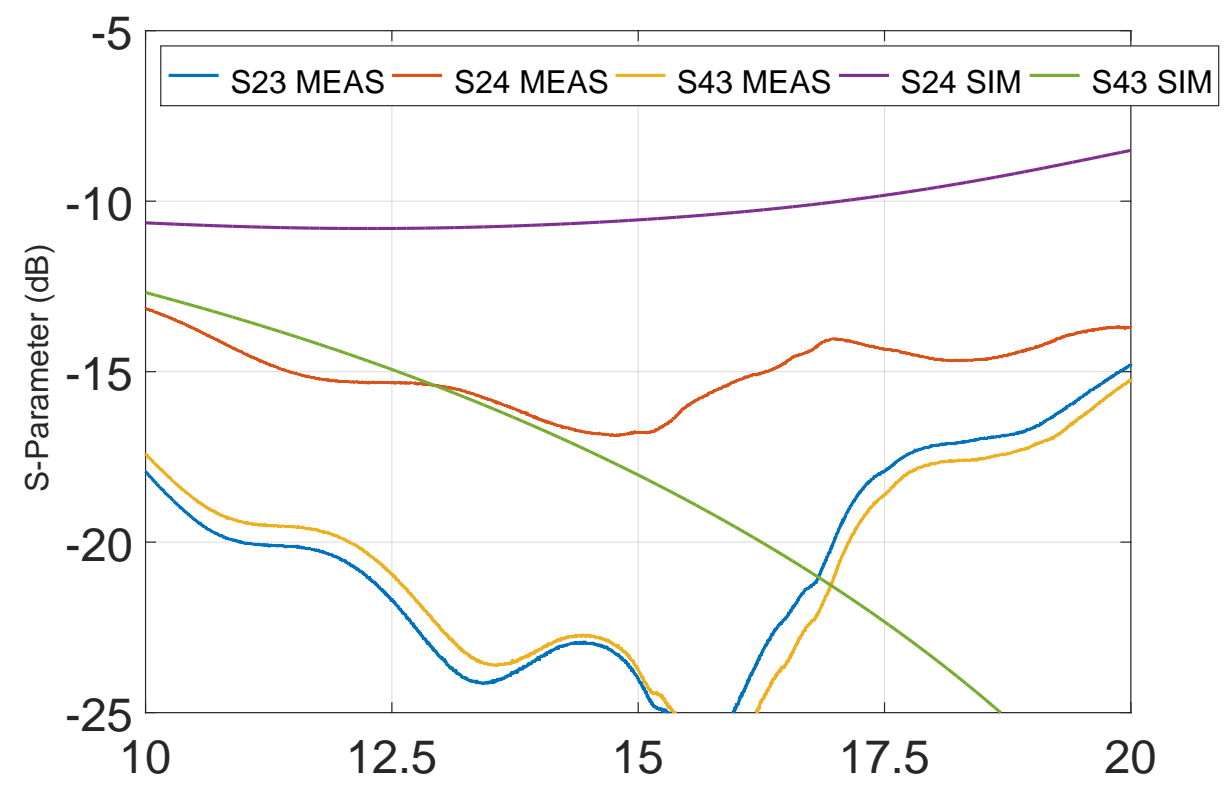

Figure 4.25: Comparison between simulated and measured isolation $\left(S_{23}, S_{34}\right.$ and $\left.S_{43}\right) S$ parameters.

\subsection{Antenna board}

The board introduced in this section encloses the TX as well as the RX antennas. The receiver antennas are directly connected to the receiver board where they are down-mixed in order to substract the information of the target following the already introduced FMCW radar procedure. A switch chain enables to select the proper TX antenna at the proper time, according to the employed TDM scheme. With the chosen MIMO array configuration an unoccupied space at the center of the array arises. This space can be used for a second functionality as for instance a camera or antennas for communication purposes as Fig. 4.26 depicts.

The available surface for the antenna array is $12 \mathrm{~cm} \times 16 \mathrm{~cm}$. Since the space limitation is very restrictive, any test coupler have been integrated before the antenna in this PCB. Moreover, some of the components are well known and they have already been utilized in the previous chapters or have been very good characterized.

An Excel sheet with the link budget is completed as Fig. 4.27 depicts. In it, the sum of the produced signal gain by the amplifiers likewise the subtraction of losses produced by the connectors, switches and microstrip lines is calculated together to estimate the overall signal level at each stage of the chain. The noise figure, which represents the degradation of the signal-to-noise ratio in the RF chain, is also included in the excell block diagram. It is calculated with the equation (3.2). Fig. 4.2 shows the position of the radiating element's phase centers and the corresponding MIMO virtual array. In this configuration a free row 


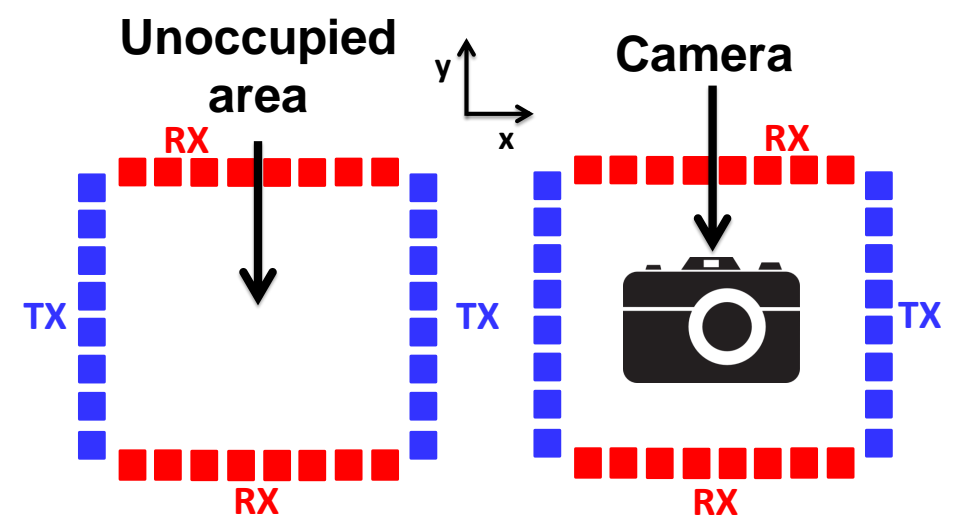

Figure 4.26: the chosen MIMO array configuration with its unoccupied space.

appears. Some authors overcame this problem from the signal processing side by linear interpolating the missing values with very good results [4].

The TX antenna element is a 2 patch array unlike the RX antenna element, which is a 4 patch array. In such a manner, the TX element size is reduced. Thus, 8 TX element fit in a single row and $8 \mathrm{RX}$ elements plus $2 \mathrm{TX}$ elements fit in a single column covering the $12 \mathrm{~cm} \times 16 \mathrm{~cm}$ available. Equations (4.13) and (4.14) calculate the angular resolution $\Delta \theta_{3 d B_{x}}$ in azimuth and $\Delta \theta_{3 d B_{y}}$ in elevation of the virtual array.

$$
\begin{gathered}
\Delta \theta_{3 d B_{x}} \approx 50^{\circ} \frac{\lambda_{0}}{N_{v i r, x} d_{x}}=50^{\circ} \frac{\lambda_{0}}{\left(2 N_{\text {pop }}+1\right) d_{x}} \approx 4.5^{\circ} \\
\Delta \phi_{3 d B_{y}} \approx 50^{\circ} \frac{\lambda_{0}}{N_{v i r, y} d_{y}}=50^{\circ} \frac{\lambda_{0}}{2 N_{\text {pop }} d_{y}} \approx 3.5^{\circ}
\end{gathered}
$$

with $\lambda_{0}$ being the wavelength in free space, $N$ the number of virtual radiating elements and $d_{x}$ and $d_{y}$ the distance between elements. Fig. 4.28 and 4.29 show the frontside and the backside of the fabricated frontend. $d_{x}$ and $d_{y}$ are $16 \mathrm{~mm}$ and $12 \mathrm{~mm}$, respectively, despite the fact that $\frac{\lambda_{0}}{2}=9.25 \mathrm{~mm}$ for the central frequency of operation. As a consequence of this, grating lobes appear. This fact was considered in the antenna array design. There is a switching network in the transmit path, which has two purposes: firstly, to provide the antennas with the desired power level. Therefore, two commercial amplifiers are used. And secondly, to direct the signal to be transmitted to individual antennas, which is needed for this MIMO radar approach. If the input power is $P_{i n}=-10 \mathrm{dBm}$, the calculated output power $P_{\text {out }}$ before the antenna is $9.7 \mathrm{dBm}$, thus the gain of the path, taking into account losses and gains from the amplifiers, lines and switches is $G_{S M}=19.7 \mathrm{~dB}$, assuming that the amplifiers are not in saturation. 


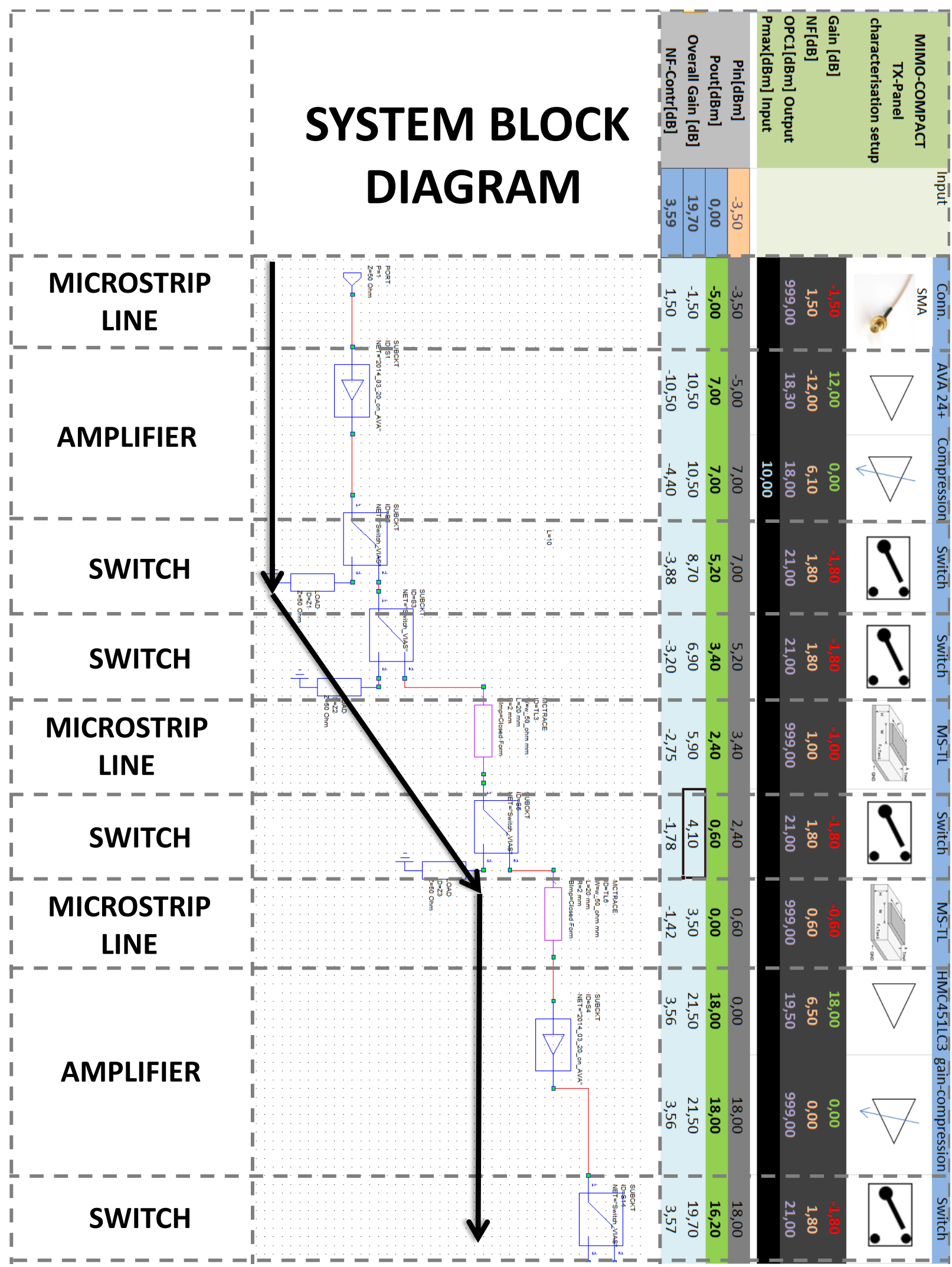

Figure 4.27: System block diagram used for simulation purposes (left-below) and estimated system power levels (right-above). 


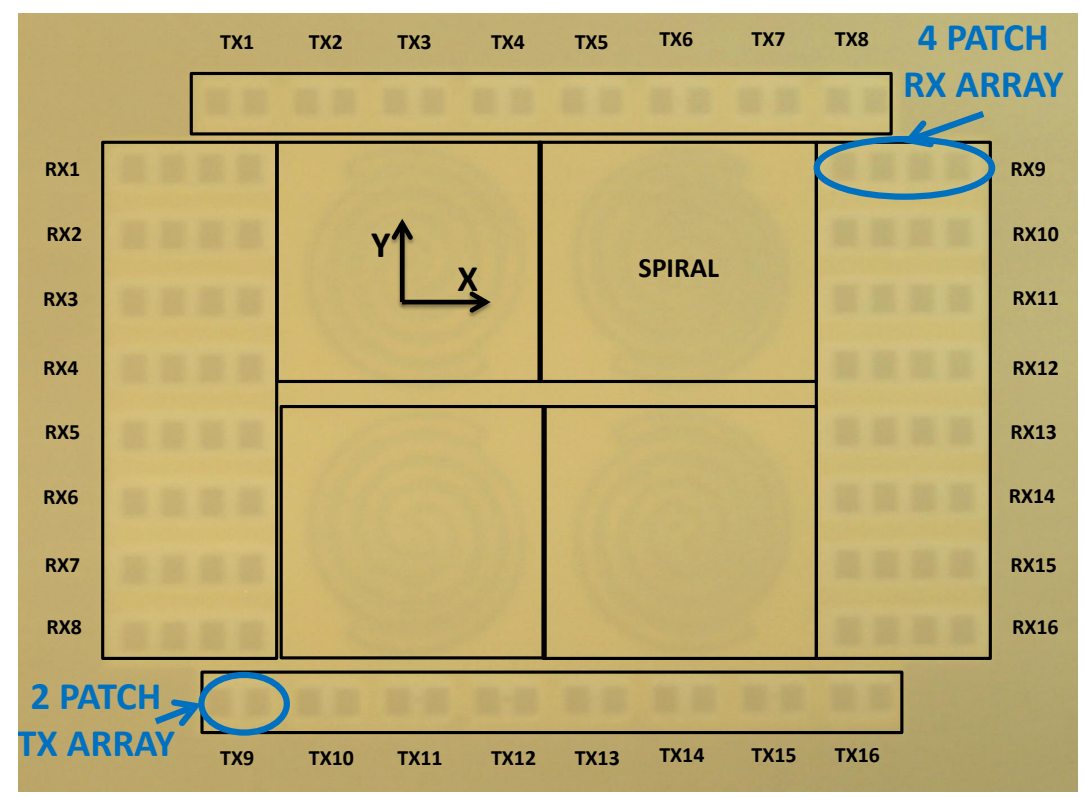

Figure 4.28: Photography of the frontend from the frontside. Patch antenna elements are only fairly visible, because they are covered by the upper substrate RO4003. The 4 Spiral antennas cannot be seen because they reside in an inner layer of the PCB.

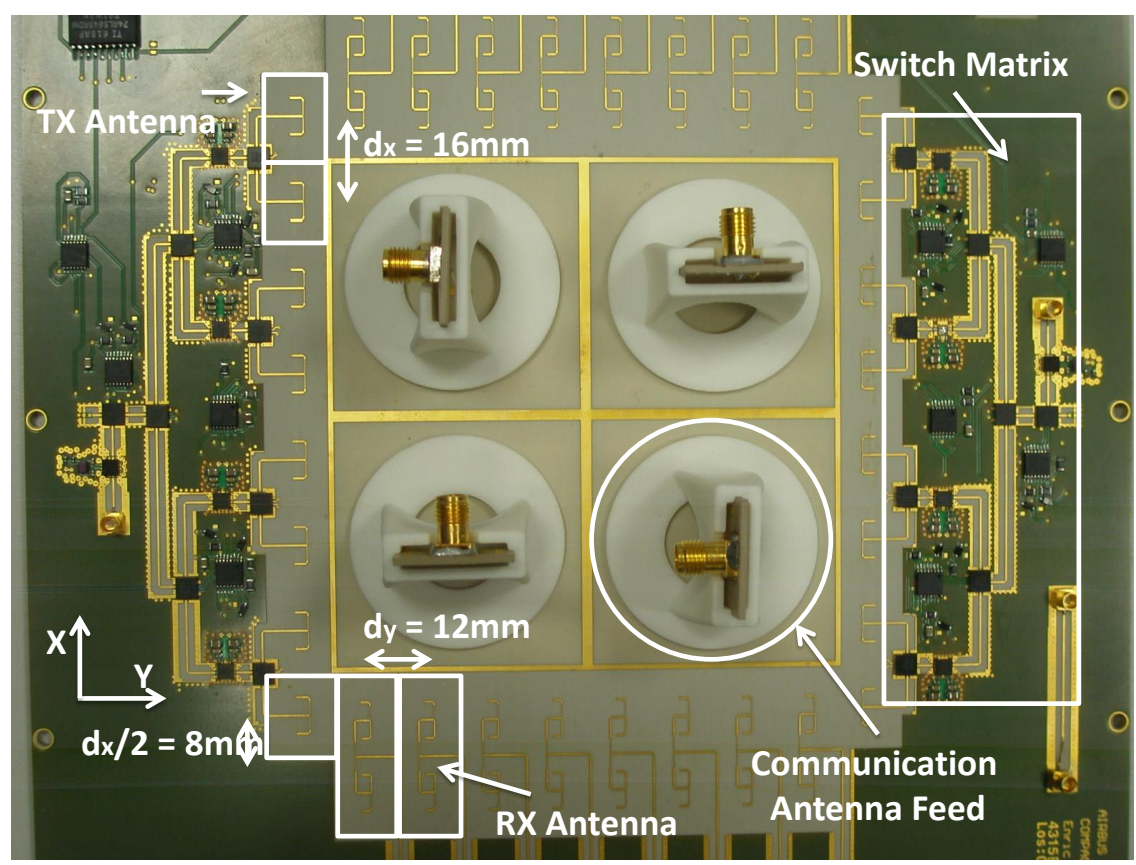

Figure 4.29: Photography of the frontend from the backside. 


\subsubsection{Antenna board: Measurements}

A 2-port network analyzer and a SOLT calibration with a standard K-connector calibration kit was performed for the spiral antennas. The spiral antennas are very broadband with matching better than $-8 \mathrm{~dB}$ from $2.25 \mathrm{GHz}$ to $4.75 \mathrm{GHz}$ covering different datalink frequency bands as well as ISM-bands at $2.4 \mathrm{GHz}$ as Fig. 4.30 .

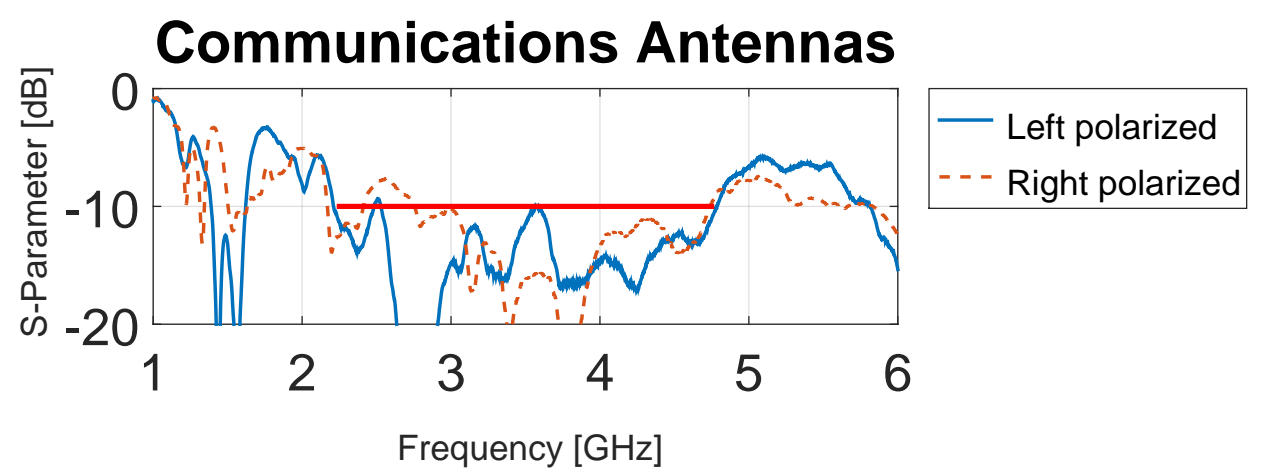

Figure 4.30: Matching of the radar and communication antennas with the specification mask.

Considering that a test coupler was not included before the antenna, the only way to calculate the power level of the board is using a test antenna. As previously mentioned, the switch matrix is designed to have a gain of $G_{S M}=19.7 \mathrm{~dB}$. In order to verify this, a measurement based on Friis' formula with a test horn antenna and a network analyzer was completed (see Fig. 4.31): the distance between antennas was $R=1.35 \mathrm{~m}$, the gain of the test antenna is $G_{R}=15.5 \mathrm{dBi}$, the gain of the RSPA is $G_{T}=7.8 \mathrm{dBi}$ (calculated from simulation).

$$
\begin{gathered}
S_{21}=P_{R}-P_{T} \\
S_{21}=G_{T}+G_{R}+G_{S M}+20 \log 10\left(\frac{\lambda_{0}}{4 \pi R}\right)=-15.4 d B
\end{gathered}
$$

Equations 4.15 and 4.16 calculate the S21 for $16 \mathrm{GHz}$, which is represented in Fig. 4.31 with a red straight line. The measurement and the calculation fit very well, hence, the simulated value of the RSPA gain and switch matrix's gain work as designed. In this section, only a selection of measurements is included. This selection does not contain the coupling between systems because, as the functionalities have different frequency band of operation, the value of the coupling is very small (under $40 \mathrm{~dB}$ ). This shows that the two functionalities can work simultaneously. 


\section{S21 - Test Horn - TX4}

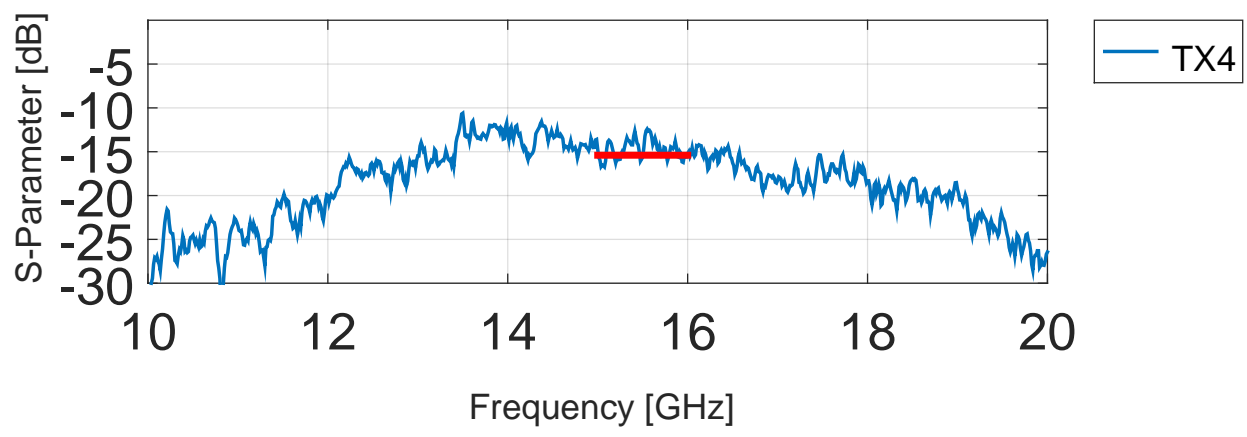

Figure 4.31: $S_{21}$ of the test set up described in text. The red line describes the theoretical value calculated with the Friis equation.

\subsection{System Performance and Image Results}

The antenna board includes in a single planar PCB, 32 radiating elements, 16 for transmit TX (in blue) and 16 for receive RX (in red). They are placed in a rectangular configuration, in such a manner, an unoccupied surface at the center of the array arises, which this time is filled with a camera. In order to achieve the desired operational bandwidth and the desired range resolution, RSPA instead of traditional patch antenna are incorporated [3]. A three way Wilkinson divider equally distributes the FMCW ramp, that is generated by the DDS and PLL, to the two independent switch chains and the receiver. The two switch chains are able to select the proper antenna at the right time or to lead the signal to a terminated port ( $50 \Omega$ resistor) in order to accomplish a orthogonality of the received data streams under a TDM paradigm. A more detailed description of the antenna board can be found in [17] [8].

The receiver board consists of 16 direct-conversion receivers. The RX signals are amplified by the LNA and then fed to the mixers. A chirp distribution network splits up the chirp signal and distributes it coherently to all 16 stages. Each stage has its own amplifier in order to drive the LO input port of the mixer. The RX signal is then mixed with the originally sent chirp and low-pass filtered. From the output of the receiver, the IF signals go directly to the digital board. The main elements of the digital board are the ZYNQ, which is a FPGA and the ARM processor in one chip, two 8-channel 14-bit ADC, which run at $100 \mathrm{MHz}$, and a Ethernet interface.

For the radar system to be compact and multifunctional, a lightweight, robust and adaptable housing is needed. To fulfill these requirements, a special housing was designed and 3D printed in aluminum. Additionally to the protection of the components, the housing is used as a heat sink. All boards are thermally linked to the housing and two cooling channels which are connected to a fan enhance the heat dissipation of the system. Fig. 4.32 shows an image of the 3D printed housing. The boxes show the TX and RX elements, 


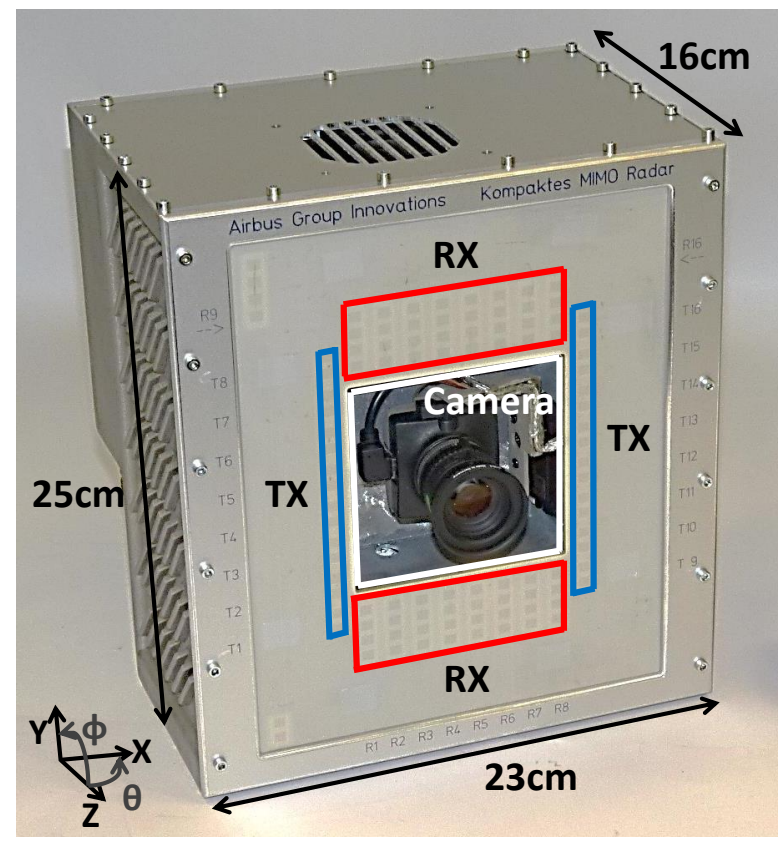

Figure 4.32: Picture of the 3D printed housing with antenna board and camera mounted in the middle. The markers show where the TX and RX antennas, and the camera are placed.

the camera and the two axis gimbal, which is mounted in the middle of the rectangular array. The size of the housing is $23 \mathrm{~cm} \times 25 \mathrm{~cm} \times 16 \mathrm{~cm}$. On the top of the housing, the opening of the cooling system can be seen.

\subsubsection{Angular resolution}

In order to measure the 3-dB-beamwidth of the system, a corner cube with a cross-section of $\sigma_{0}=150 \mathrm{~m}^{2}$ is placed in an anechoic chamber, at a range $R=23.1 \mathrm{~m}$, with azimuth and elevation angles of $\phi=0^{\circ}$ and $\theta=0^{\circ}$, respectively. With the FFT processing and FMCW ramp settings presented in former sections, a three-dimensional radar image, which contains the information of range, azimuth and elevation, is calculated.

Fig. 4.33 shows the normalized azimuth $\left(\phi=0^{\circ}\right)$ and elevation $\left(\theta=0^{\circ}\right)$ profiles taken at the range cell of the central target. Under the assumption that the corner cube is a point target and the distance is big enough to be in the far-field region, the theoretical values of the angular resolution of the MIMO virtual array (calculated in Section 4.5) should match with the measured 3-dB-beamwidth, shown in Fig. 4.33. The measured azimuth resolution is $\Delta \theta_{3 d B_{x}}=4.7^{\circ}$ and the elevation resolution is $\Delta \phi_{3 d B_{y}}=3.6^{\circ}$ whereas the calculated is $\Delta \theta_{3 d B_{x}}=4.5^{\circ}$ and the elevation resolution is $\Delta \phi_{3 d B_{y}}=3.5^{\circ}$. As it can be seen, the measured and calculated angular resolutions match very well. One thing to consider is that in the estimation along the azimuth direction the missing element is included, while 


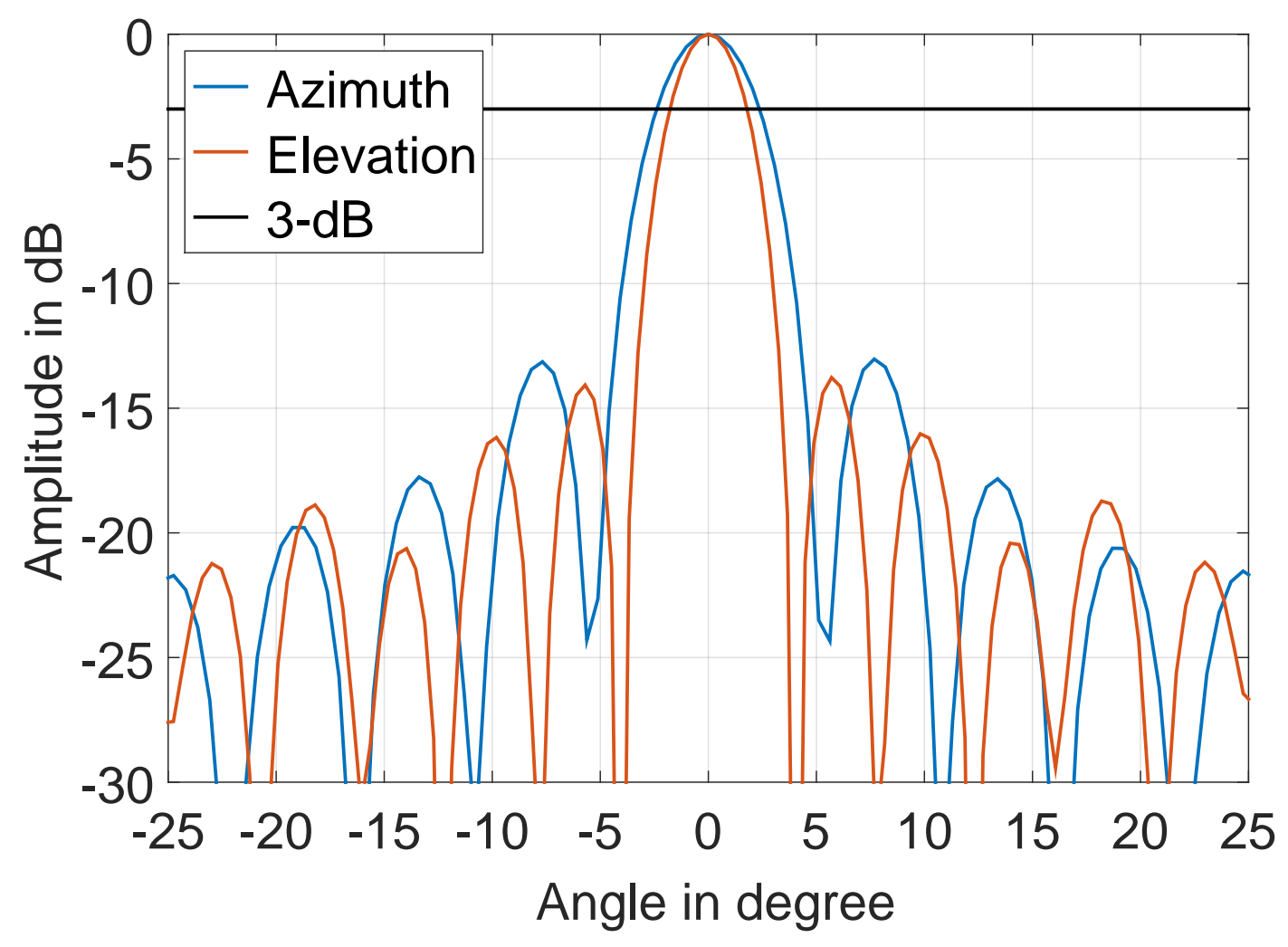

Figure 4.33: Azimuth and elevation profiles for a central target.

in the radar beam-forming process, this missing element is calculated as the average of its neighbors.

\subsubsection{Radar field of view}

The aim of this sub-chapter is to show the dependency between a target's received power, at a certain distance $R$, and its angle, in both azimuth and elevation directions. A corner cube with a cross-section of $\sigma_{0}=150 \mathrm{~m}^{2}$ is placed at $R=23.1 \mathrm{~m}, \phi=0^{\circ}$ and $\theta=0^{\circ}$. Then, the radar is rotated by $\phi=-60^{\circ}$ in elevation direction, without moving the target. At this point, a radar capture is started. This process is repeated following an angular sweep from $\phi=-60^{\circ}, \theta=0^{\circ}$ to $\phi=60^{\circ}, \theta=0^{\circ}$, with a constant step of $1^{\circ}$. Since the RX (array of 4 patches along y) and TX (array of 2 patches along y) antennas are not isotropic, a relatively pronounced dependency of the received power with the angle is expected.

Fig. 4.34 illustrates the measured curves for different angles $\phi=\phi_{i}, \theta=0^{\circ}$. The simulated curve, dotted curve in the figure, is the multiplication of the simulated RX 


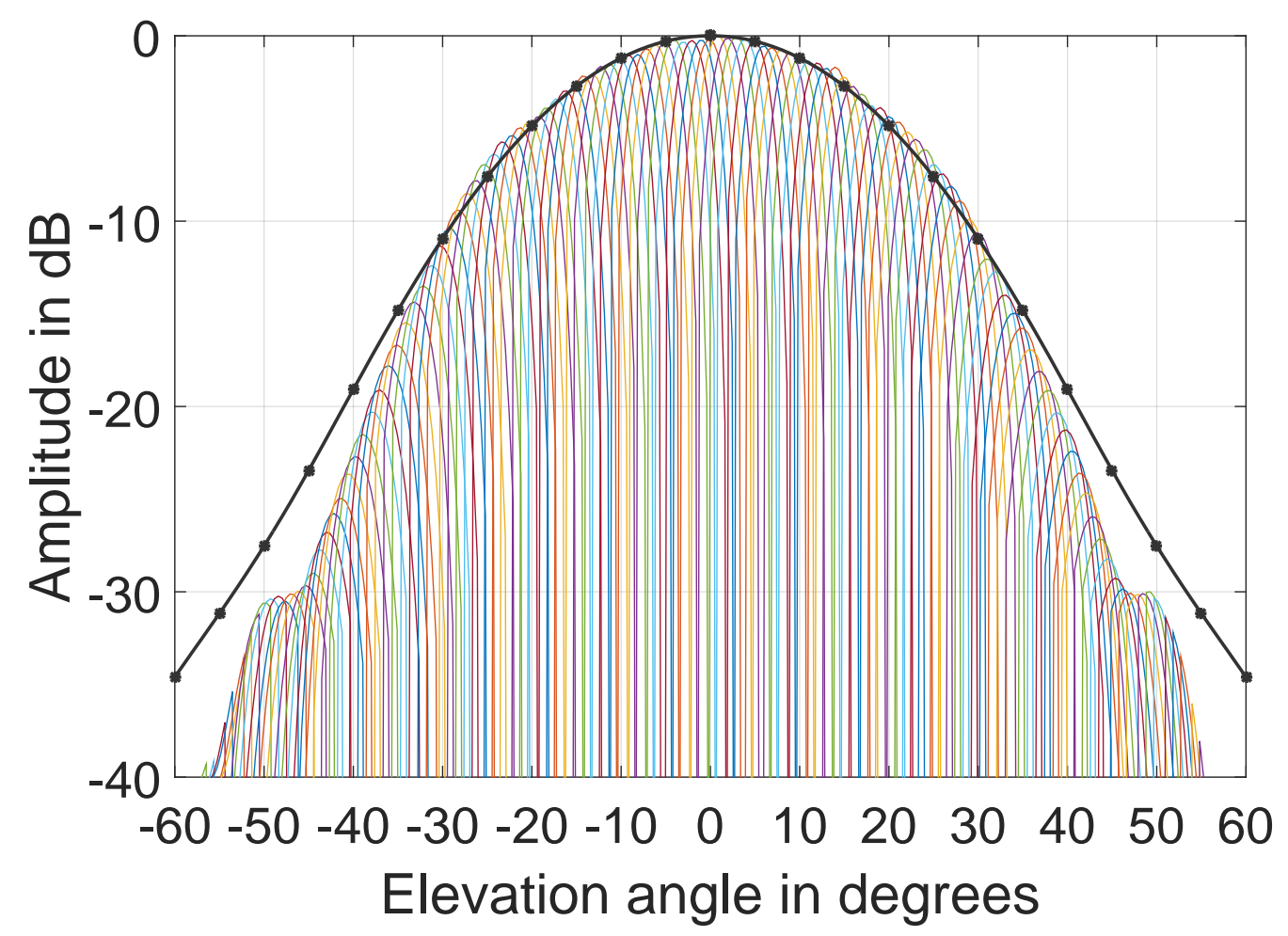

Figure 4.34: Normalized dependence of the received power in the elevation direction with the angle due to the antenna element pattern.

antenna pattern and the TX antenna pattern. As it can be seen, the amplitude decays approximately by $\pm 10 \mathrm{~dB}$ at $30^{\circ}$. The simulation matches very well with the envelope of the complete set of performed measurements.

Similarly, the measurement has been performed in the azimuth direction. This time the angular sweep is from $\phi=0^{\circ}, \theta=-60^{\circ}$ to $\phi=0^{\circ}, \theta=60^{\circ}$, with a constant step of $1^{\circ}$. Since the RX (single patch along $\mathrm{x}$ ) and TX (single patch along $\mathrm{x}$ ) antennas are not isotropic, a corresponding angular dependence of the power is expected. In this axis, the antennas are single patches Therefore, the characteristic dependence of the received power with the angle is expected to be rather wide.

Fig. 4.35 illustrates the measured curves for different angles $\left(\phi=\phi_{i}, \theta=0^{\circ}\right)$. Considering the TDM architecture implemented, each TX radiating element is connected to a switch, in such a manner that only the TX antenna which sends the FMCW ramp is matched to $50 \Omega$, while the rest of the TX antennas are connected to a open-circuit. Consequently, a ripple appears in the azimuth dimension. The simulated curve, dotted curve in the figure, is the multiplication of the simulated RX antenna pattern and the TX 


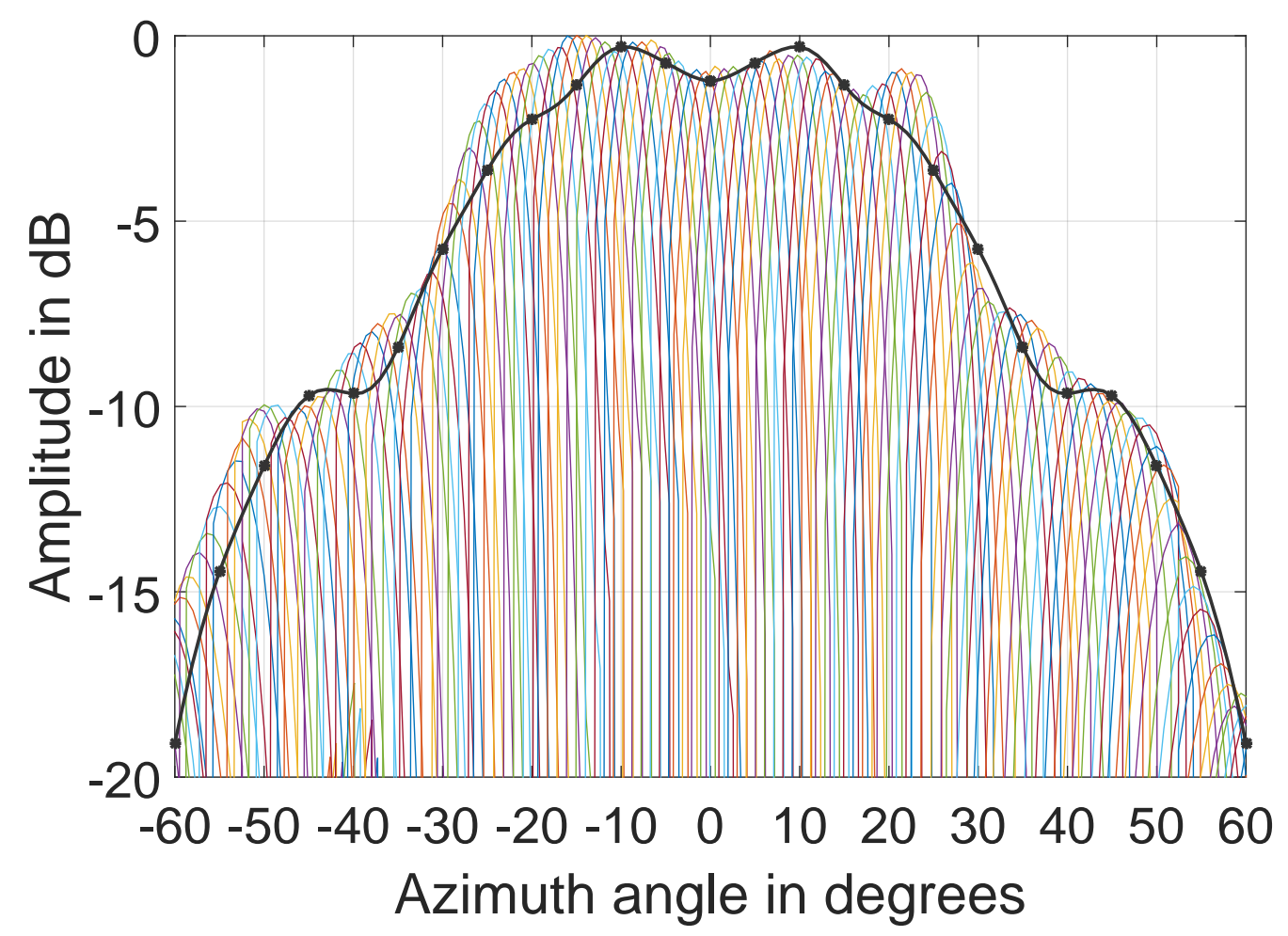

Figure 4.35: Normalized dependence of the received power in the azimuth direction with the angle due to the antenna element pattern.

antenna pattern (considering the whole antenna array with the corresponding open circuits in the proper TX elements). The amplitude decays approximately by $10 \mathrm{~dB}$ at $\pm 50^{\circ}$. The simulation matches rather well with the envelope of the complete set of performed measurements. As a summary, the fields of view of the radar are $F O V_{\theta}=100^{\circ}$ and $F O V_{\phi}=60^{\circ}$, respectively.

\subsubsection{Zone surveillance applications}

The next step is to verify the performance of the MIMO radar in a real scenario and to show the advantages of a multifunctional system. In this test, the MIMO radar is combined with a camera which is placed in the middle of the antenna array. Fig. 4.36 shows the scene of the measurement as seen by the camera. A commercial camera, with $n_{p i x, h}=2048$ pixels horizontally and $n_{p i x, v}=1536$ pixels vertically is used. The pixel size is $3.2^{\circ}$. With an objective with a focal length of $f=12 \mathrm{~mm}$, a field of view of

$$
F O V_{h}=\frac{n \cdot p}{f} \cdot \frac{360^{\circ}}{2 \pi}=31.3^{\circ}
$$




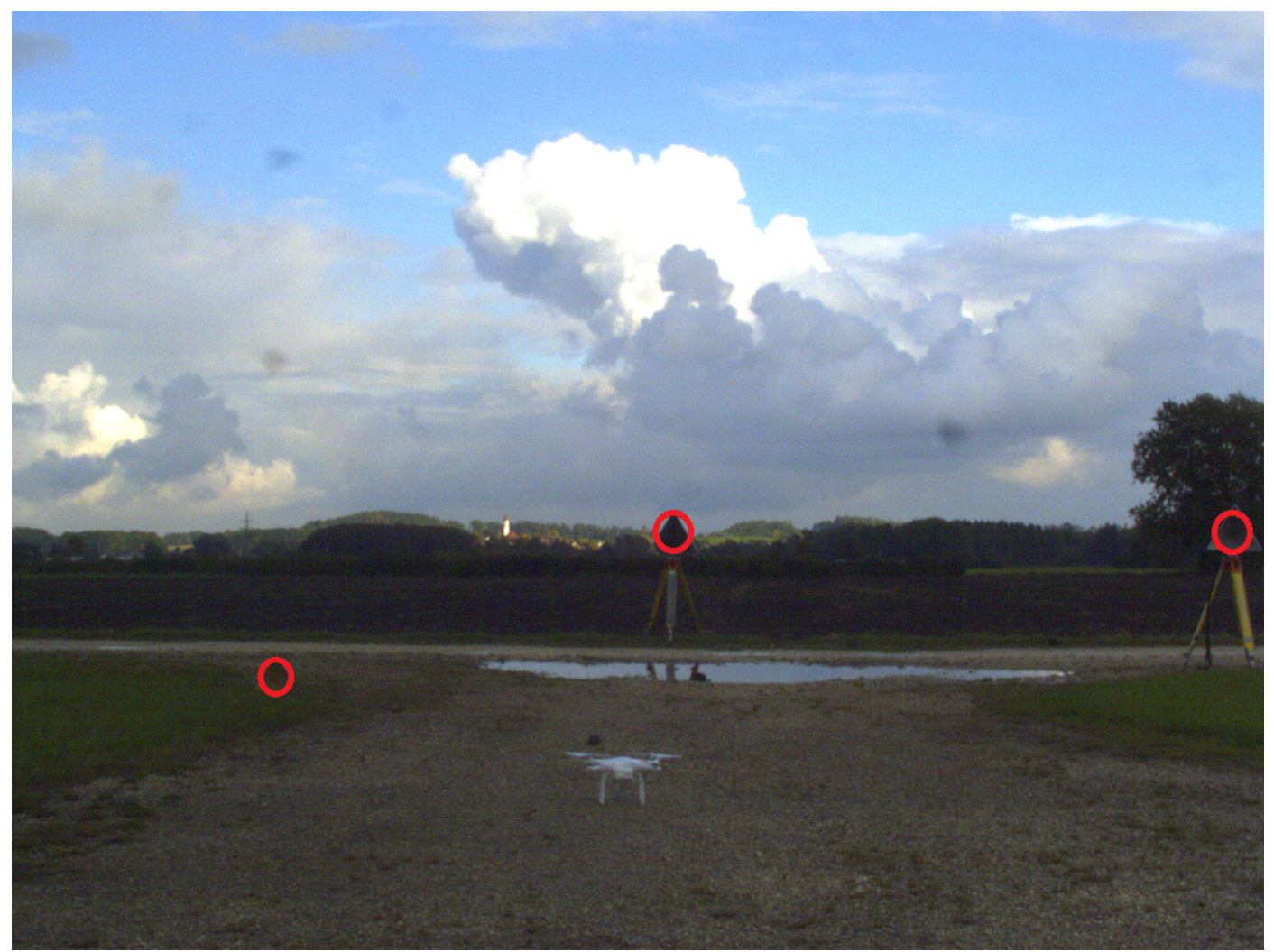

Figure 4.36: Image of the scene with three corner cubes marked with red circles.

horizontally and $F O V_{v}=23.5^{\circ}$ vertically is obtained. The field of view of the radar have been calculated in the previous sub-section to be $F O V_{\phi}=60^{\circ}$ and $F O V_{\theta}=100^{\circ}$. Since the FOV of the radar is greater than the FOV of the camera, the gimbals can be used to point the camera to the right direction. A field test measurement has been performed, in order to simulate a detectable change in the surveillance area. For this purpose, three corner cubes with cross-sections $\sigma_{1} \approx 49 \mathrm{~m}^{2}, \sigma_{2} \approx 422 \mathrm{~m}^{2}$ and $\sigma_{3} \approx 169 \mathrm{~m}^{2}$ are placed at ranges of $18 \mathrm{~m}, 28 \mathrm{~m}$ and $22 \mathrm{~m}$, respectively. Determining the accurate position of the objects in a medium range is a computationally intensive task for pure image processing algorithms.

The results of the 3D-FFT beamforming radar processing is shown in Fig. 4.37. As it can be seen, a correct estimation of the range, azimuth and elevation, for all 3 targets used in the measurement is achieved.

An additional and more intuitively understandable image, fusion of the camera image and the radar data is presented in Fig. 4.38. Here, the camera image is superimposed with the radar data. In order to still see the scene, the amplitude of the reflection is used to define the transparency level and the color of the radar cell. With this method, strong reflections occur as opaque areas in the image. In this case, the evaluation is done for the 


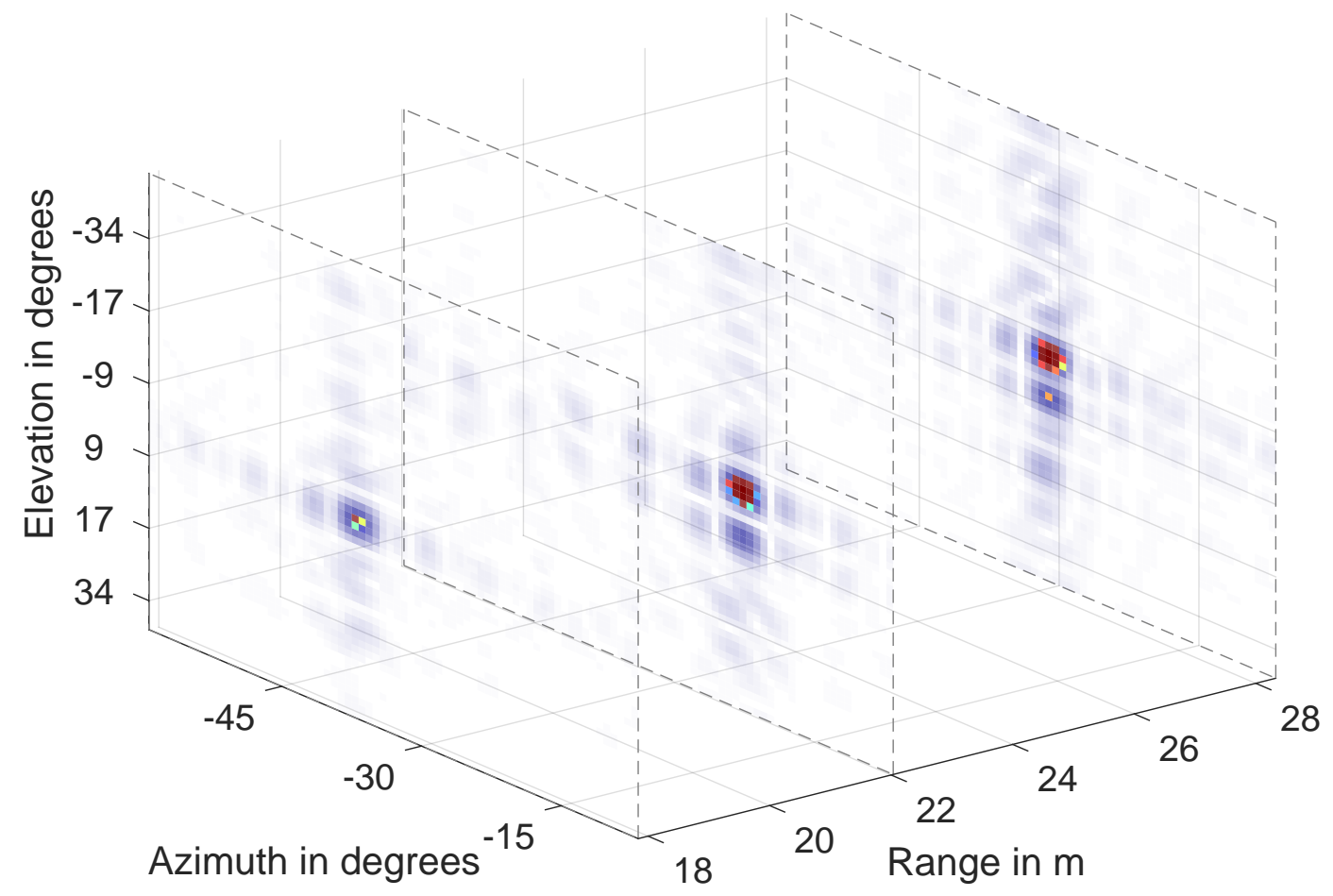

Figure 4.37: 3D reconstruction of the scene with radar data. The three targets are spaced in range and have different positions in azimuth and elevation.

three most significant targets in amplitude. According to the distance, a red, a yellow and a green tag are placed close to the targets, where the red tag shows the closest, and usually most important, target. This way of displaying the information is very intuitive since a strong target within the monitored scene is highlighted in the camera image. Hence, a higher degree of automation is achieved. Another aspect to remark is that the system is compact and portable and thus can be easily integrated into existing infrastructures.

Another application is the detection and classification based on image processing. In this case the radar can be combined with a zoom camera pointed to the objects previously detected by the radar. Therefore, a different camera objective is needed, e.g. with a focal length of $f=75 \mathrm{~mm}$, which leads to a field of view of $F O V_{h}=5.0^{\circ}$. This is comparable to the azimuth resolution of the radar, which makes it possible to observe each azimuth sector separately. To point the camera, the two axis gimbal can be used in combination with the already implemented tracking algorithm. The servos of the camera gimbal are linked to the radar processing and receive the azimuth and elevation angles of the most significant target. This could be the one with the highest amplitude or the one which is closest to the 


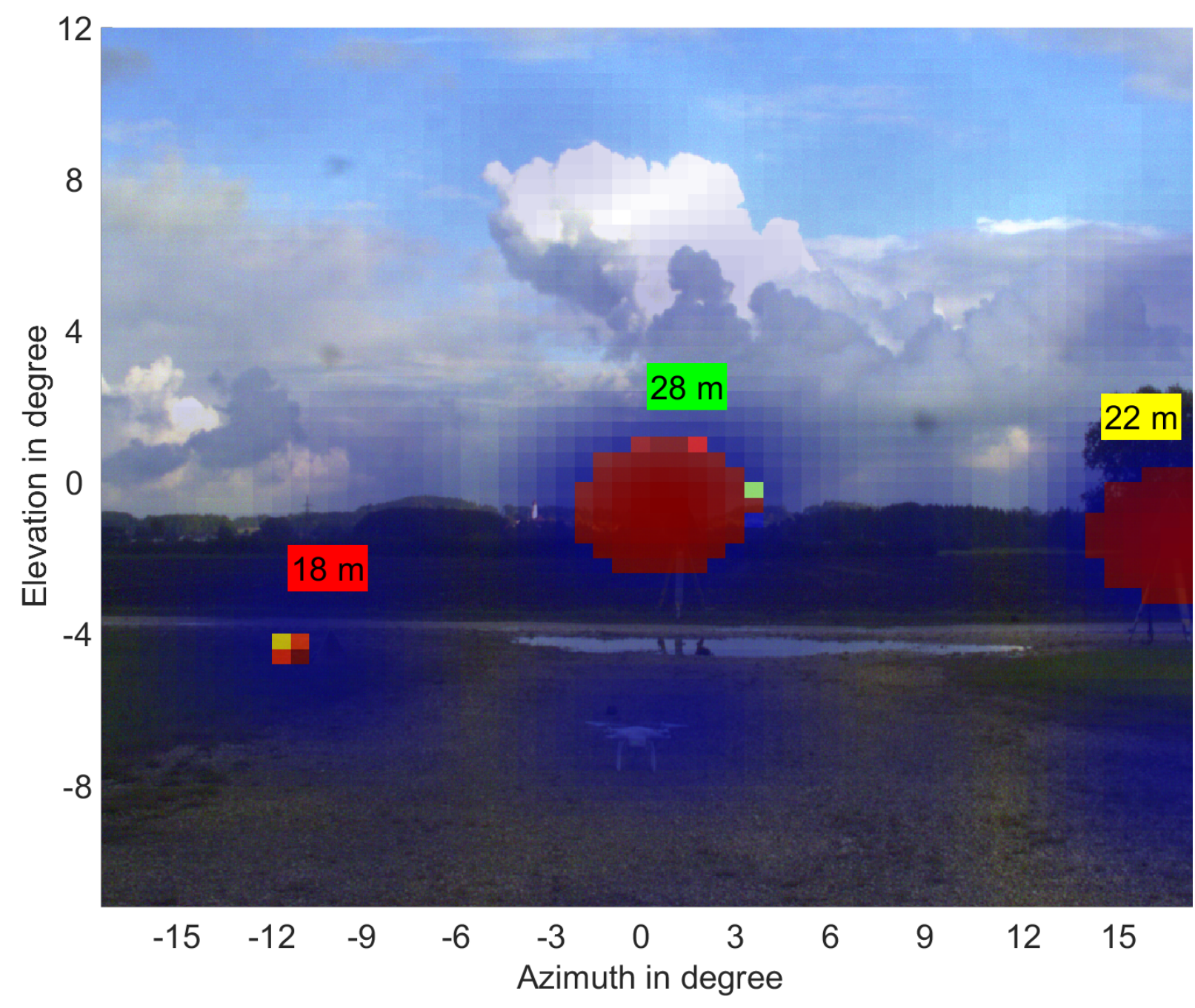

Figure 4.38: Camera and radar image overlaid. The color and the level of opaqueness is proportional to the amplitude of the reflection. The targets' ranges are displayed in boxes with different colors. The field of view is limited by the camera.

radar. The gimbal then moves to the target's direction and offers a much more confined area to be processed by the image processing. The zoom increases the resolution of the target. Object detectors in image processing need a certain minimum number of pixels, on which they can operate. When using a wide-angle objective, the resolution of a target in medium distance would be too low. With a zoom objective the resolution is increased and the probability of detection is higher and a better classification can be performed. 


\subsection{Final conclusions}

In this chapter a second proof of concept of a complete MIMO radar is presented. It successfully reduces the volume of the last prototype from $(55 \mathrm{~cm} \times 27 \mathrm{~cm} \mathrm{x} 27 \mathrm{~cm})$ to $(16 \mathrm{~cm} \mathrm{x} 25 \mathrm{~cm} 23 \mathrm{~cm})$. The costs are also diminished due to the use a single PCB for the antennas. Furthermore, the system was integrated with two different functions, antennas for communications and a gimbal holding a camera. The device has demonstrate to be suitable for the targeted application (change detection in wide zones such as nuclear plants). The performance of the multi-functional system is verified by the measurements in a multi-target environment showing synergies among the two functionalities. The system is able to perform a 3D reconstruction of the surroundings via the MIMO radar, extracting range azimuth and elevation of the targets, and combining this information with a camera image, or sending it through the integrated communication system. This technological approach has a high potential for these applications in which the space limitation is critical as for instance, aid in the landing operation or the object classification of flying platforms as helicopters or UAVs. 


\section{Bibliography}

[1] Y. Lo, D. Solomon and W. Richards, "Theory and experiment on microstrip antennas", in IEEE Transactions on Antennas and Propagation, vol. 27, no. 2, pp. 137-145, Mar 1979 .

[2] D. M. Pozar, "Microstrip antenna aperture-coupled to a microstripline", in Electronics Letters, vol. 21, no. 2, pp. 49-50, January 171985.

[3] F. Croq and A. Papiernik, "Large bandwidth aperture coupled microstrip antenna", in Electronics Letters, vol. 26, pp. 1293-1294, August 1990.

[4] T. Spreng, Y. Shuai V. Valenta, H. Schumacher, U. Siart and V. Ziegler, "Wideband $120 \mathrm{GHz}$ to $140 \mathrm{GHz}$ MIMO radar: System design and imaging results", in IEEE Microwave Conference (EuMC), 2015 European, Paris, France, Sep 2015.

[5] A. Kishk, "Advancement in Microstrip Antennas with Recent Applications", in INTECH, First published March, 2013.

[6] D. M. Pozar "A review of Aperture Coupled Microstrip Antennas: History, Operation, Development, and Applications", May 1996.

[7] I. J. Bahl and D. K. Trivedi "A Designer's Guide to Microstrip Line", Microwaves, May 1977, pp. 174-182.

[8] H. A. Wheeler "Transmission-line properties of a strip on a dielectric sheet on a plane", in IEEE Tran. Microwave Theory Tech. MTT, vol. 25, no. 8, pp. 631-647, Aug 1977.

[9] L. Zhu and K. Wu, "Ultrabroad-band vertical transition for multilayer integrated circuits", in IEEE Microwave and Guided Wave Letters, vol. 9, no. 11, pp. 453-455, Nov 1999.

[10] M. Leib, M. Mirbach and W. Menzel,"An ultra-wideband vertical transition from microstrip to stripline in PCB technology", in 2010 IEEE International Conference on Ultra-Wideband, Nanjing, 2010, pp. 1-4.

[11] Lei Zhu and W. Menzel, "Broad-band microstrip-to-CPW transition via frequencydependent electromagnetic coupling", in IEEE Transactions on Microwave Theory and Techniques, vol. 52, no. 5, pp. 1517-1522, May 2004. 
[12] Lei Zhu and W. Menzel, "Broad-band microstrip-to-CPW transition via frequencydependent electromagnetic coupling", in IEEE Transactions on Microwave Theory and Techniques, vol. 52, no. 5, pp. 1517-1522, May 2004.

[13] X. Huang and K. L. Wu, "A Broadband and Vialess Vertical Microstrip-to-Microstrip Transition", in IEEE Transactions on Microwave Theory and Techniques, vol. 60, no. 4, pp. 938-944, April 2012.

[14] F. P. Casares-Miranda, C. Viereck, C. Camacho-Penalosa and C. Caloz, "Vertical microstrip transition for multilayer microwave circuits with decoupled passive and active layers", in IEEE Microwave and Wireless Components Letters, vol. 16, no. 7, pp. 401403, July 2006.

[15] E. Wilkinson "An n-way hybrid power divider". IEEE Trans. Microw. Theory Techn., vol.8, no. 1, pp. 116-118. Jan. 1960.

[16] J. C. Chiu, J. M. Lin and Y. H. Wang, "A Novel Planar Three-Way Power Divider", in IEEE Microwave and Wireless Components Letters, vol. 16, no. 8, pp. 449-451, Aug. 2006.

[17] E. Miralles, D. Rieth, B. Schoenlinner, U. Prechtel, A. Meusling, A. Belenguer, H. Esteban and V. Ziegler, "Low Cost Multipurpose RF System Approach: Integration of a MIMO Radar and a Communications Frontend on a Single PCB", in IEEE Microwave Conference (EuMC), 2016 European, London, UK, Oct 2016.

[18] E. Miralles, T. Multerer, A. Ganis, B. Schoenlinner, U. Prechtel, A. Meusling, J. Mietzner, C. Weckerle, H. Esteban, M. Vossiek, M. Loghi, and V. Ziegler, "Multiductional and Compact 3D FMCW MIMO Radar System with Rectangular Array for Medium Range Applications" IEEE Aerospace and Electronic Systems Magazine. Accepted. 


\section{Chapter 5}

\section{Very wideband components for radar applications.}

Multifunctional systems which contain different functionalities within their specific frequency bands are highly desired and one of the aims for future products develpment in Airbus, notably in the aeronautic industry [1], because they provide size, weight and power (SWaP) reduction. There are two possibilities of combining these systems: an integration of different RF-frontends for each application or a single multi-band and multifunction RF-frontend which covers the needed part of the electromagnetic spectrum. The main advantage of the first approach is that all RF-functionalities can work simultaneously without interfering each other.Furthermore, the International Telecommunication Union (ITU) divided the world into three regions with different frequency bands allocated to terrestrial broadcasting services (see Fig. 1.3). Usually, a radar product must be developed at least three times according to the frequency regulation. A broadband radar which covers the desired frequency bands is a good solution to this problem. Moreover, a broadband radar itself has multiple advantages: a larger operational bandwidth of the signals usually leads to a higher range resolution and allows frequency hopping techniques [3]. Furthermore, if the power of the signal is distributed over a larger frequency band, the probability of intercepting the radar decreases [4].

The focus of this chapter is to introduce novel RF very broadband components as a 3D printed TEM horn antenna [5] and a Wilkinson power splitter [6]. The targeted frequency band of operation is from $5 \mathrm{GHz}$ to $40 \mathrm{GHz}$. This wide frequency range can not be easily achieved with a concatenation of resonant structures, which only work for a single frequency. Tapered transmission lines, which are very broadband "per se" are key structures for the work presented in this chapter. The initial frequency of operation depends on the length of the structure while the final frequency of operation depends on the fabrication tolerances, since ideal taper structures present high pass characteristic. Furthermore, these components must be built or integrable with a well-known technology as PCB. 


\subsection{Basics of Traditional Wilkinson Dividers}

The Wilkinson power splitter was invented more than 50 years ago by Ernest Wilkinson. This device splits a signal into at least two equal phase signals. It is a symetric reciprocal device, in such a way that it also combines two equal-phase signals into one in the opposite direction. A loss-less reciprocal three-port network cannot have all ports simultaneously matched [8], for this reason Wilkinson included a resistor between its arms. A resistor with the proper value $\left(2 Z_{0}\right.$ when the impedance of the arms is $\left.\sqrt{2} Z_{0}\right)$ not only matches the three ports but also fully isolates port 2 from port 3 (see Fig. 5.1). Furthermore, the resistor does not add resistive loss to the power split from port 1.

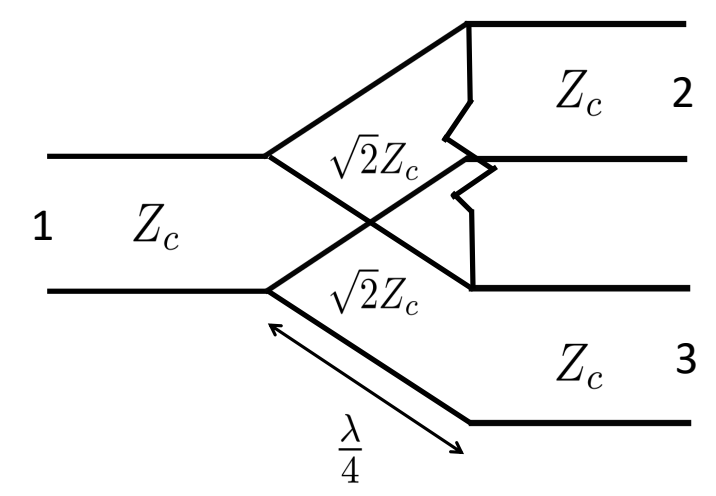

Figure 5.1: Circuit model of a traditional Wilkinson divider.

\subsection{Broadband Wilkinson}

For many RF-systems, power dividers are key components that are used to combine or split a signal. On one hand, classical T-junctions accomplish this task in a simple way, on the other hand, they have poor isolation, a fact that makes T-junctions less popular in modern microwave circuits. Although E. J. Wilkinson presented a new power divider [7] solving the isolation problem, a traditional Wilkinson divider uses a $\frac{\lambda}{4}$ impedance transformer thus, it is a rather narrow band device. In order to achieve more bandwidth, more resistors can be added. The characteristic line impedance of the transmission line increases in each step. If a low cost compact and well known transmision line technology such as microstrip is used, to increase the characteristic line impedance of the transmission line means to reduce the width of the microstrip. Widths smaller than $w=0.1 \mathrm{~mm}$ (value that is needed depending on the substrate and the desired bandwidth) present a problem for standard fabrication processes of printed circuit boards. This problem can be bypassed by reducing the line's impedance before the split, but it is not an optimal solution since the size of the circuit and its losses increase. 
Recently, a new tapered power divider was presented [9]. The authors replaced the $\frac{\lambda}{4}$ impedance transformer with a tapered line. This increases the bandwidth avoiding the microstrip width issue. However, if a very large bandwidth is needed, the number of design parameters and, hence, the complexity of the problem, increase. A genetic algorithm was used in order to design a TWD [10. The whole process was performed with a full wave simulation achieving a bandwidth of $B W=8 \mathrm{GHz}$ with 2 resistors. Another approach used non-uniform transmission lines to reduce the arm's length [11]. This work presents a fast design method with an equivalent circuit model, which is roughly 33 times faster than using a full wave simulation. This method is verified by a proof-of-principle for a very wideband tapered Wilkinson divider, which works between 2 and $38 \mathrm{GHz}$. It is reusable and suitable for multiband applications.

\subsubsection{Improvements of Tapered Wilkinson Divider}

TWDs have mainly two advantages over classical Wilkinson dividers. The first one is that tapered lines present a broadband performance, as compared to a $\lambda / 4$ stepped impedance matching section. The second one is that once the taper function $Z(z)$ is designed (see figure 5.2), it can be fixed while more resistors are added $(Z(z)$ means the characteristic impedance of the transmission line at the distance $\mathrm{z}$ from the bifurcation).

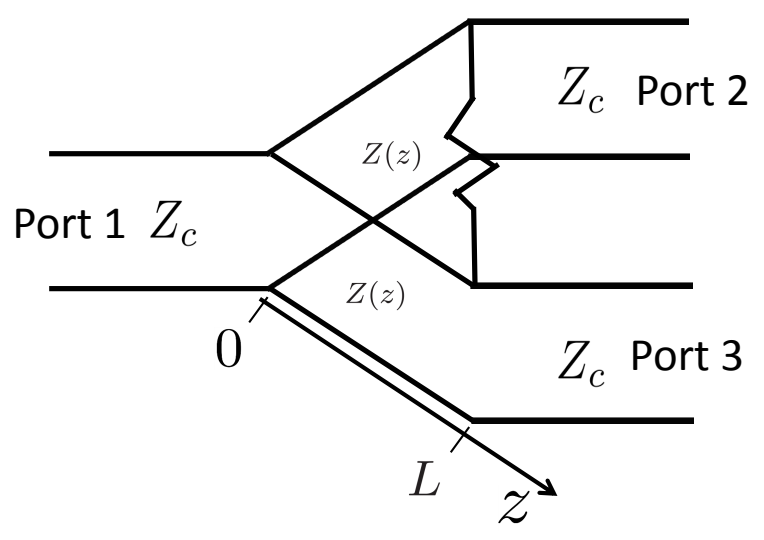

Figure 5.2: Circuit model of a tapered Wilkinson divider.

In order to match the arm from both sides, $Z(0)=2 Z_{c}$ and $Z(L)=Z_{c} . Z(z)$ and $L$ are the design parameters that define the lowest frequency of operation $f_{0}$ from which on the taper is well matched. Figure 5.3 shows the simulation of the tapered line with and without pads, which are needed for soldering the resistors. A lot of scientific literature deals with the topic of optimum design of tapered lines [12] [13] [14]. In this work, the focus is on improving the S-parameters at ports 2 and 3. 


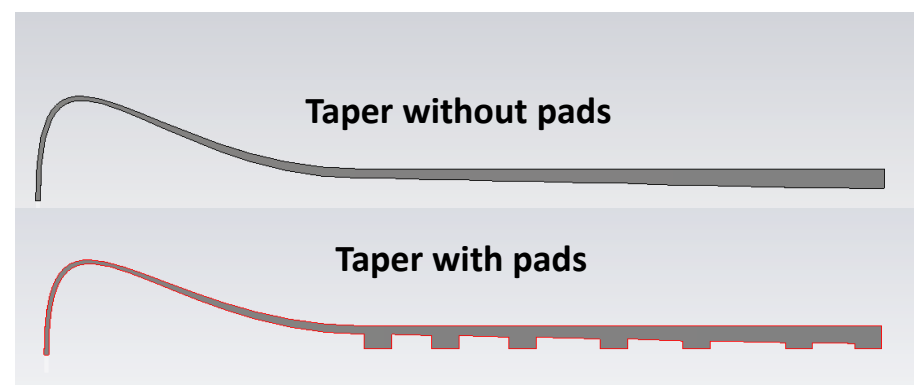

Taper Reflection

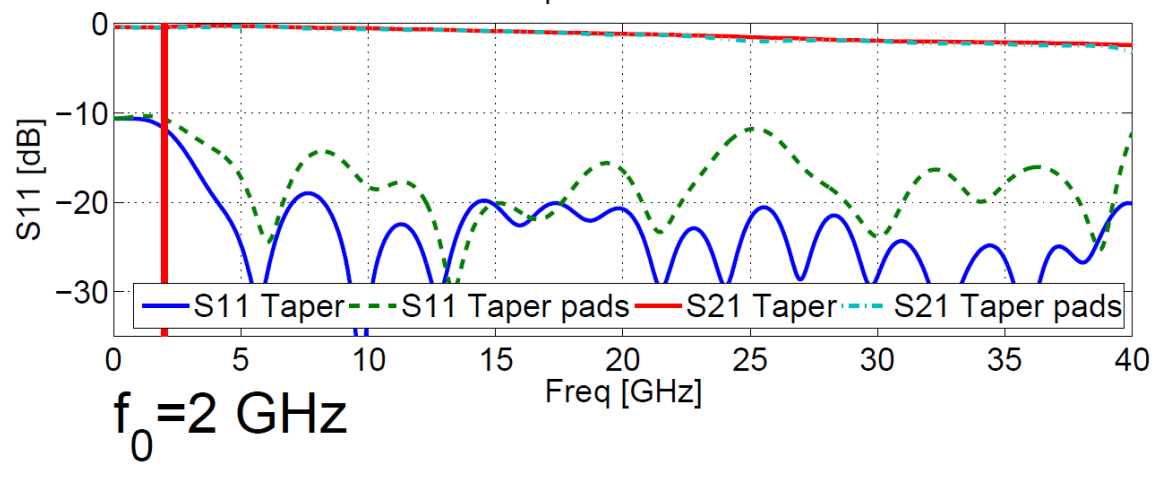

Figure 5.3: Comparison between the simulated taper with and without pads.

\subsubsection{Circuit model}

In this section, a circuit model which calculates isolation and reflection at the TWD's arms is presented. By using superposition and circuit symmetry principles, the even/odd mode analysis is applied. In order to calculate the final $S_{22}$ and $S_{23}$ (or $S_{33}$ and $S_{32}$ since the network is reciprocal and symmetrical) of the device, the reflection factors of the even and odd modes ( $\rho_{\text {even }}$ and $\rho_{\text {odd }}$ ) need to be determined (see equations (5.1) and (5.2)).

$$
\begin{aligned}
& S_{33}=S_{22}=\frac{1}{2}\left(\rho_{\text {even }}+\rho_{\text {odd }}\right) \\
& S_{23}=S_{32}=\frac{1}{2}\left(\rho_{\text {even }}-\rho_{\text {odd }}\right)
\end{aligned}
$$

The even mode becomes a virtual open in the symmetry plane. Figure 5.4 shows the even mode equivalent circuit model. The circuit is matched $\left(\rho_{\text {even }}=0\right)$ for the operation frequency range of the tapered line (see figure 5.3), thus $Z_{\text {even } 2}=2 Z_{c}, Z_{\text {even } 1}=Z_{c}$ independently on the number of resistors, considering that they are connected to an open circuit.

The odd mode becomes a virtual short in the symmetry plane. Figure 5.5 shows the 


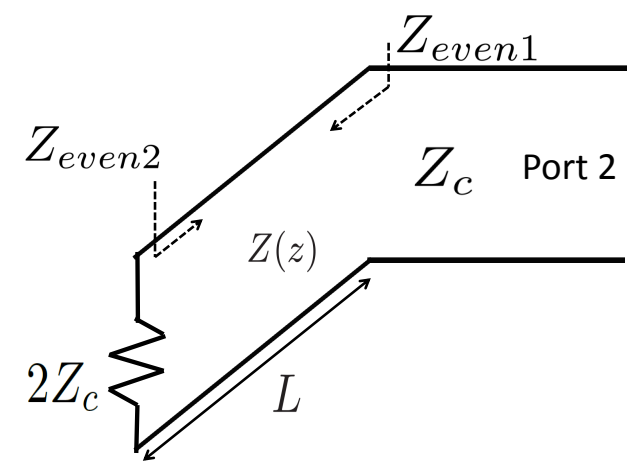

Figure 5.4: Even mode equivalent circuit model.

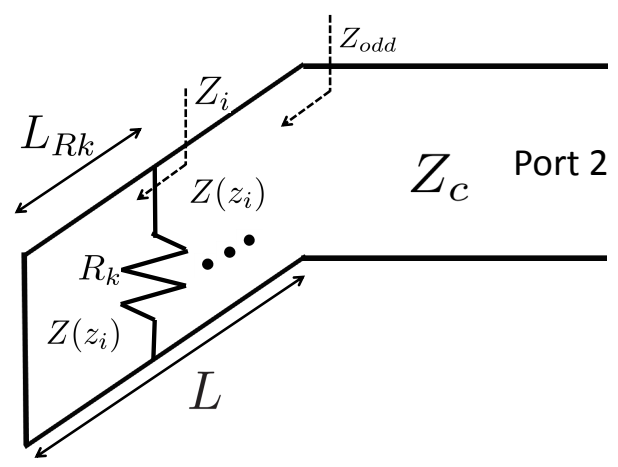

Figure 5.5: Odd mode equivalent circuit model with $N$ resistors.

odd mode equivalent circuit model. From equation (5.3), the reflection factor of the odd mode $\rho_{\text {odd }}$ can be calculated if the impedance seen from port $2, Z_{\text {odd }}$, is known.

$$
\rho_{\text {odd }}=\frac{Z_{\text {odd }}-Z_{c}}{Z_{\text {odd }}+Z_{c}}
$$

Equation (5.4) represents the impedance $Z_{i}$ seen (figure 5.5) from an arbitrary space coordinate $z_{i}$. $i$ varies between $0<i<M+1$ and represents the index of the discrete sampled value.

$$
Z_{i}=\left\{\begin{array}{cl}
Z\left(z_{i}\right) \frac{Z_{i-1}+Z\left(z_{i}\right) j \tan (\beta \Delta L)}{Z\left(z_{i}\right)+Z_{i-1} j \tan (\beta \Delta L)} & \text { if } z_{i} \neq L_{R k} \\
Z_{i-1} / / R_{k}=\frac{Z_{i-1} R_{k}}{Z_{i-1}+R_{k}} & \text { otherwise }
\end{array}\right.
$$

The characteristic impedance function of the taper, $Z(z)$ needs to be sampled in order to be processed with the computer. For this reason, it is denoted as $Z\left(z_{i}\right)$. $\Delta L$ is the distance between discrete samples while $R_{k}$ is half of the value of a resistor (for reason of symmetry) placed at a distance $L_{k}$ from the short circuit (see figure 5.5). The index 
$k$ represents a resistor and it varies between $0<k<N+1$, where $N$ is the number of resistors. As we can see in figure 5.5, the impedance seen from $z_{1}=0$ is a short circuit while the impedance seen from $z_{M}$ is equal to the impedance $Z_{\text {odd }}$ seen from the port.

$$
\begin{gathered}
Z_{1}=0 \\
Z_{M}=Z_{\text {odd }}
\end{gathered}
$$

Once the reflection factors for the odd and even cases are calculated, the S-parameters of ports 2 and 3 can be obtained from equations (5.1) and (5.2). As previously mentioned, $\rho_{\text {even }}=0$ at the operation frequency band of the tapered line while:

$$
\begin{gathered}
S_{33}=S_{22}=\frac{1}{2}\left(\rho_{\text {even }}+\rho_{\text {odd }}\right)=\frac{\rho_{\text {odd }}}{2} \\
S_{23}=S_{32}=\frac{1}{2}\left(\rho_{\text {even }}-\rho_{\text {odd }}\right)=-\frac{\rho_{\text {odd }}}{2}
\end{gathered}
$$

With this circuit model, the S-parameters can be calculated as a function of the values, the position and the amount of resistors. The more resistors are added the more degrees

of freedom equation (5.4) has. Consequently (since $\beta=\frac{2 \pi f}{c}$ ), the more frequencies are matched or the better values for isolation and reflection are achieved. Note that this model is based on ideal transmission lines, valid for every transmission line technology and not considering microstrip particuliarities.

\subsubsection{Fast Design Method}

The design parameters of a TWD are the number of resistors N, their position in the tapered line $L_{k}$ and their value $R_{k}$. The way to achieve wide bandwidth is to increase the number of resistors, which also increases the number of design parameters and thus, the computational complexity. Consequently, a time efficient method which does not rely only on full wave simulation is needed. In this paper, the proposed circuit model (very fast method) is used in order to calculate a first set of $N, R_{k}$ and $L_{k}$, whereas the full wave simulator determines the final value of $L_{k}$. Figure 5.6 shows a flow chart with the steps of the method.

\section{Optimization with circuit model}

Once the specifications are defined, an optimization is launched using the direct algorithm [15] for finding the global minimum of a multivariate function subject to simple bounds on the variables such as the ones included in equations (5.1) and (5.2). The values of $N, R_{k}$ and $L_{k}$ are optimized until $S_{22}$ and $S_{32}$ are conformal with the specifications. Note that only available values of resistors $R_{k}$ are considered. 


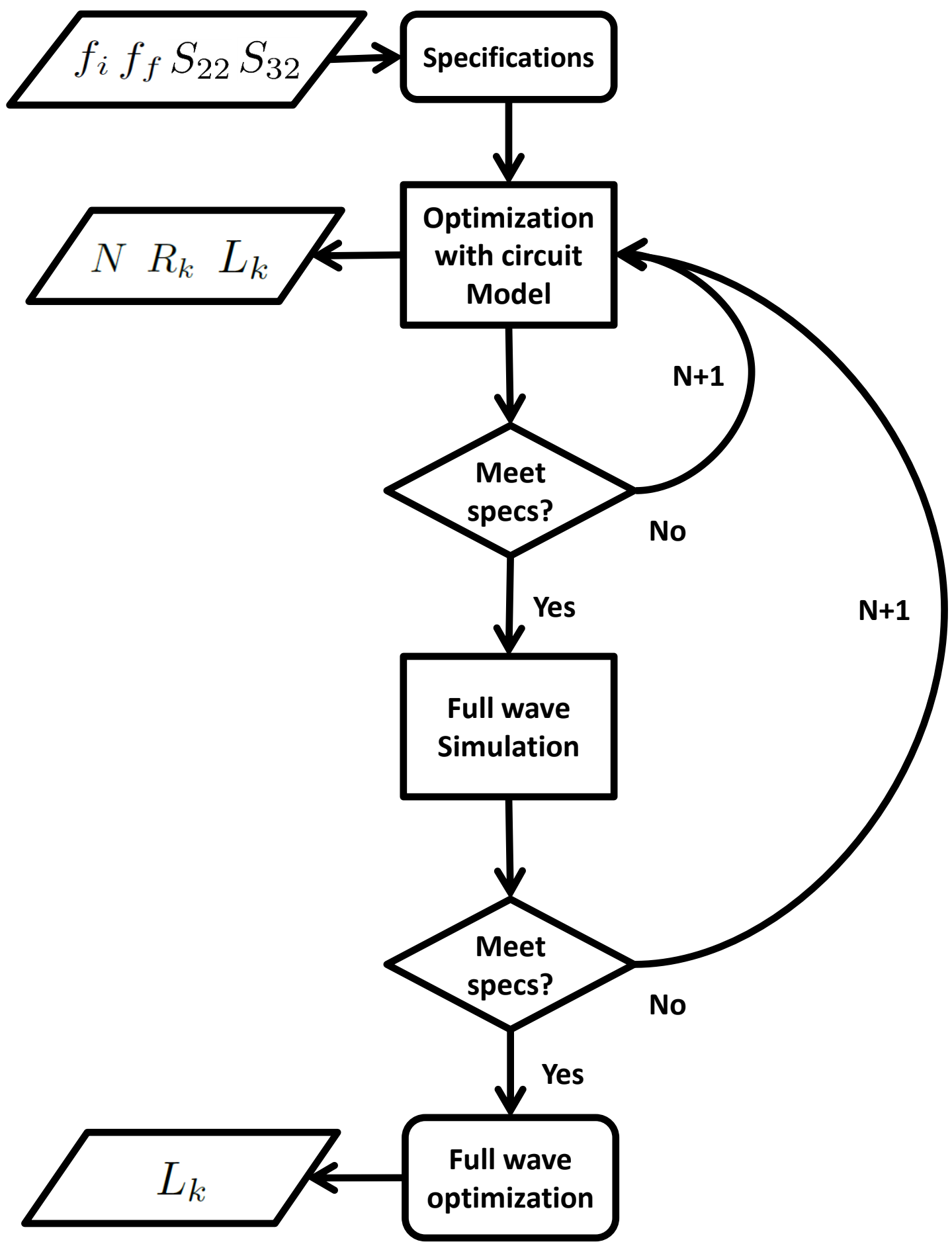

Figure 5.6: Flow chart of the design method. 


\section{Full wave simulation}

After the optimization with the circuit model, a full-wave simulation is started in order to take the losses, the coupling between the branches and the pads for soldering the resistors into account. If the S-parameters match the specifications, the next step is done without increasing the number of resistors.

\section{Full wave optimization}

At the end, a full wave optimization is performed only with the position of the resistors $L_{k}$ to be optimized. The number of resistors and their values remain the same in this step saving computational time.

\subsubsection{Measured Results}

The design method was used to design a very wideband $(2-38 \mathrm{GHz})$ TWD. A substrate with $\epsilon_{r}=3.55$, height $h=0.203 \mathrm{~mm}$ and $\tan (\delta)=0.0027$ was utilized. The width of the microstrip with $50 \Omega$ is $w_{50}=0.422 \mathrm{~mm}$ while the one with $100 \Omega$ is $w_{100}=0.1 \mathrm{~mm}$. The tapered lines vary their impedances smoothly between $100 \Omega$ and $50 \Omega$ through a line lenght of $L=23.95 \mathrm{~mm}$. Figure 5.7 illustrates the fabricated and measured device, while table 5.1 shows the position $L_{k}$ and the value $R_{k}$ of the seven resistors needed.

Table 5.1: Position and value of the resistors

\begin{tabular}{cccccccc}
$k$ & 1 & 2 & 3 & 4 & 5 & 6 & 7 \\
\hline$R_{k}[\Omega]$ & 200.0 & 200.0 & 200.0 & 200.0 & 175.0 & 100.0 & 50.0 \\
$L_{k}[\mathrm{~mm}]$ & 23.65 & 21.8 & 18.93 & 16.52 & 13.93 & 11.62 & 9.55
\end{tabular}

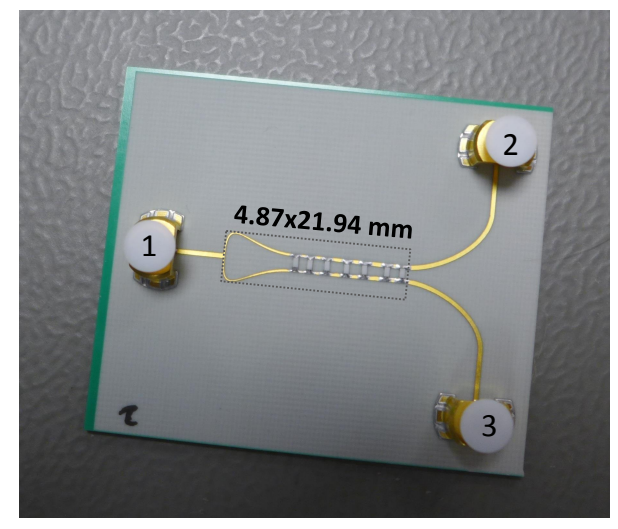

Figure 5.7: Photography of the fabricated device.

A 2-port network analyzer and a termination with matching better than $-25 \mathrm{~dB}$ was utilized to measure the S-parameters of the fabricated device. As figures 5.8 and 5.9 show, 
the measurement fits rather well the simulation. The circuit fulfills specification's mask achieving: $S_{11}<-10 \mathrm{~dB}, S_{22}<-12 \mathrm{~dB}, S_{23}<-10 \mathrm{~dB}$ and $-3.4<S_{21}<-6.0 \mathrm{~dB}$.

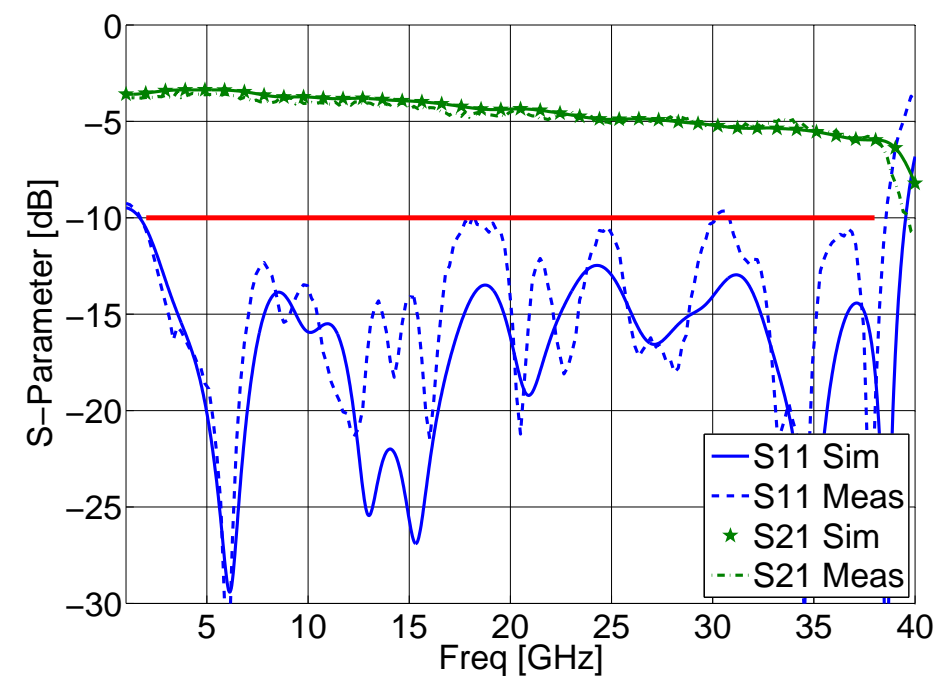

Figure 5.8: Comparison between measured and simulated $S_{11}$ and $S_{21}$. The figure also shows the specification's mask.

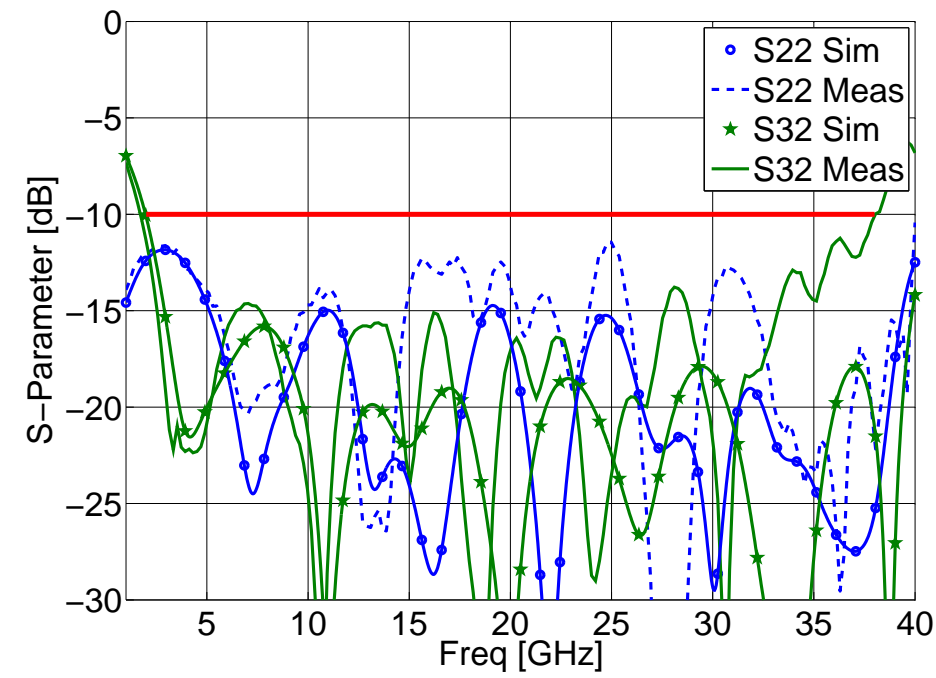

Figure 5.9: Comparison between measured and simulated $S_{22}$ and $S_{32}$. The figure also shows the specification's mask.

Table 5.2 shows a comparison between the main parameters of the TWD presented in this paper and several other published TWDs. The presented device achieves around 4.5 
Table 5.2: Comparative results of main parameters withother published results.

\begin{tabular}{lcccc} 
& Ref [9] & Ref [10] & Ref [11] & This work \\
\hline Length [mm] & 29.65 & 18.00 & 10.00 & 21.94 \\
Operation band [GHz] & $2.0-10.2$ & $2.5-10.6$ & $3.1-10.6$ & $2-38.0$ \\
Bandwidth [GHz] & 8.2 & 8.1 & 7.5 & 36.0 \\
Number of resistors & 3 & 2 & 3 & 7 \\
Ripple of $S_{21}[\mathrm{~dB}] /$ IL & $3.1-3.5$ & $3.1-3.5$ & $3.2-3.8$ & $3.4-6.0$ \\
Worst case reflection [dB] & 15 & 15 & 13 & 10 \\
Isolation [dB] & 15 & 15 & 13 & 10
\end{tabular}

Table 5.3: Summary of time complexity

\begin{tabular}{cccc} 
Full wave (s) & Full wave (iter) & Model (s) & Model (iter) \\
\hline 199228 & 111 & 6055 & 1637
\end{tabular}

times more bandwidth of operation than already published TWDs with a comparable size in the same transmission line technology (microstrip). Table 5.3 shows a summary of the computational time needed to do the various simulation and optimization steps.

\subsection{A 3D Printed PCB integrated TEM Horn Antenna}

3D printing is revolutionizing the industry [16] with many applications for different sectors such as the medical [17], automotive [18], construction [19], and aeronautic [20]. Some of the benefits of 3D printing are fast prototyping capability, design flexibility, low material waste, no middleman (from the printer to the user) and the possibility of cost and weight reduction. Over the last years, the scientific community has paid attention to and spent effort on combining this new technology with Radio Frequency (RF) applications. Proof of that is the increasing number of recently published articles related to this topic as for example a 3-D printed patch antenna [21], a vertical transition on a multilayer printed substrate [22], a multilayer MSL and a dipole antenna [23], dielectric reflect arrays [24], a traditional horn antenna [25] and a lens-antenna [26].

Multi-functional RF systems [27] 28] provide Size, Weight and Power (SWaP) reduction compared to conventional systems and often rely on very broadband operation. However, broadband antennas that can conveniently be integrated in PCBs, like Tapered Slot Antenna, often radiate in the perpendicular direction of the PCB. If a $2 \mathrm{D}$ array is needed then they cannot be integrated in a single PCB and a sandwich configuration with multiple stacked PCBs is required. Accordingly, the costs and the volume of the overall system would increase.

In this work, a 3D printed TEM Horn antenna [29], which can be integrated with PCBs 


\subsection{A 3D Printed PCB integrated TEM Horn}

and radiates normal to the PCB plane is developed, designed, fabricated and measured. This antenna enables to build a very broadband 2D antenna array with potential benefits for SWaP and cost.

\subsubsection{Basics of TEM Horn Antennas}

The TEM horn antenna is a very popular antenna. The antenna consists of only two metal plates and a feeding structure. The operational principle is similar to a taper, in such a way that the lenght of the antenna determines the initial working frequency while the final frequency of operation is related with the fabrication tolerancies. On the other hand, the feeding structure can also restringe its performance over frequency.

The radiation properties of TEM horns can be modified by varying the flare and the plate width of the horn. In order to maximize the efficiency of the antenna the mode matching between the feed and free space must be ensured. Several autors have proposed a variety of new horn structures such as linear, exponential, Klopfenstein, triangular, Hecken and superelliptic tapers. The superelliptic function has shown to maintain the main lobe centered over frequency and to present a very large impedance bandwidth [32].

$$
\begin{gathered}
x(t)=480 \sin ^{1.4} t \\
z(t)=120.5-120 \cos ^{0.7} t
\end{gathered}
$$

Eq. (5.9) and (5.10), where $0<\mathrm{t}<\pi / 2$ is a parameter which defines the plate separation. In this work a superelliptic function has been used to design the fabricated TEM horn antenna.

\subsubsection{Antena concept}

Fig. 5.10 depicts a sketch of the antenna and its transition to MSL. The main beam of the antenna radiates in the broadside direction $\hat{z}$ as Fig. 5.10A shows. The antenna is made up of two conductive parts which are fastened together on the top and the bottom of a PCB with a dielectric screw in order to avoid short-circuits (see Fig. 5.10B). Fig. 5.10C and Fig. 5.10D illustrate the bottom view with the transition from MSL to PPW and the sideways view with the $90^{\circ} \mathrm{PPW}$ bend, respectively. To fabricate the two conductive parts with a standard direct machining tool is not possible due to their geometrical complexity. Therefore, 3D printing is used, although the roughness is relatively high in comparison with other fabrication methods. 


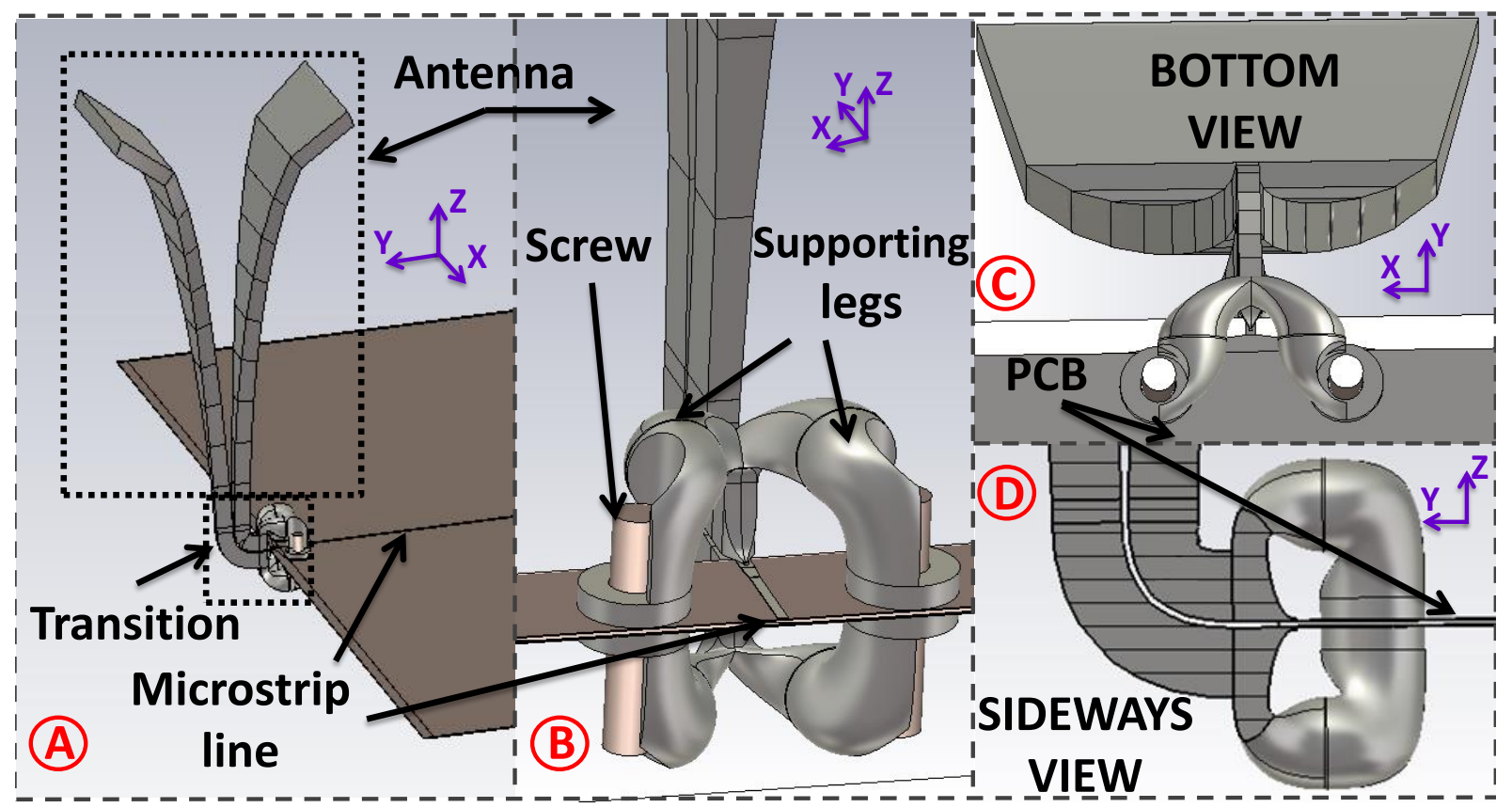

Figure 5.10: Sketch of the proposed antenna. A. General view. B. Focus on the transition. C. Bottom view. D. Sideways view.

\subsubsection{MSL to PPW transition}

In this section the designed transition from a MSL to PPW is described.

\section{Back-to-back transition within a PCB}

The selected dielectric material for the PCB has an electrical permittivity $\epsilon_{r}$ of 3.55 , loss tangent $\tan (\delta)$ of 0.0027 and height $h$ of $203 \mu \mathrm{m}$. The width of the MSL $w_{m s}$ is $0.422 \mathrm{~mm}$ and of the PPW $w_{p p w 1}$ is $0.580 \mathrm{~mm}$. They are calculated with the classical wheeler equations [30] to have an impedance of $50 \Omega$. Both transmission lines are connected via a tapered structure with a length of $L_{t 1}=1 \mathrm{~mm}$. The ground plane of the MSL and a strip conductor of the PPW are joined on one face of the dielectric sheet and the other two strips are united on the opposite face (see Fig. 5.11 and Fig. 5.13).

A back-to-back PCB transition from a microstrip line to PPW (filled with dielectric material) is fabricated and tested. It must be notice that this transition doesn't include any $3 \mathrm{D}$ printed part since it is only an intermediate step. The transition has a length $L_{\text {Line }}$ of $20 \mathrm{~mm}$.

Fig. 5.12 depicts the simulated and measured S-parameters of the described transition. The simulation and the measurement of the S-parameters present good agreement. The reflection is less than $-10 \mathrm{~dB}$ almost over the whole frequency range of interest. This is an acceptable value considering that the measured back-to-back transition has two transitions and its influence in the final structure is approximately $6 \mathrm{~dB}$ less. Moreover the short tapered transition $L_{t 1}=1 \mathrm{~mm}$ produces small mismatches, which are responsible for the 


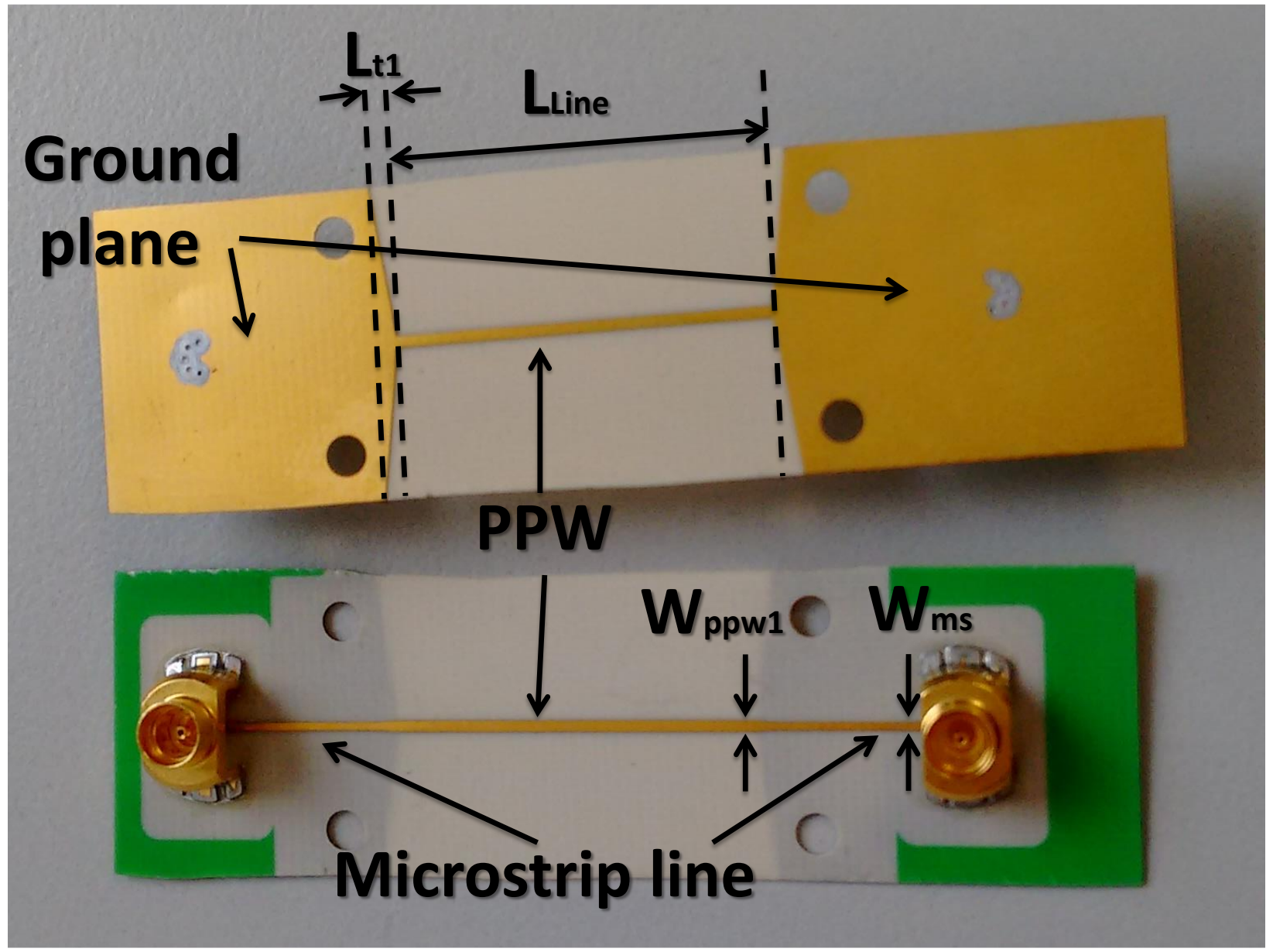

Figure 5.11: Photography of the back-to-back transition on the PCB with the main geometrical parameters.

small resonances that the Insertion Loss (IL) plot shows. These small resonances can be reduced by lengthening the tapered structure. As a consequence, the transition would become larger.

\section{Back-to-back transition including a 3D Printed structure}

A PPW with a length of $L_{t 2}=1 \mathrm{~mm}$ is included, in order to connect the 3D printed parts, which have a width of $w_{p p w 2}=1.33 \mathrm{~mm}$ (see Fig. 5.14). The remaining air gap between plates $S_{\text {gap }}=h+2 t$ is $0.273 \mathrm{~mm}$, where $t=0.035 \mathrm{~mm}$ is the thickness of the metal layer on the PCB.

The PPW parts are printed in AlSi10Mg (Aluminium alloy with conductivity between $\sigma=16-21 \mathrm{MS} / \mathrm{m})$ with an accuracy of $\pm 100 \mu \mathrm{m}$ with a EOS M270 machine. It operates with Direct Metal Laser Sintering (DMLS) technology. However, more accurate results are achieved depending on the shape and printing position of the part. A back-to-back transition is printed with a length of $L_{p p w}=33 \mathrm{~mm}$ (see Fig. 5.14). The air gap is 


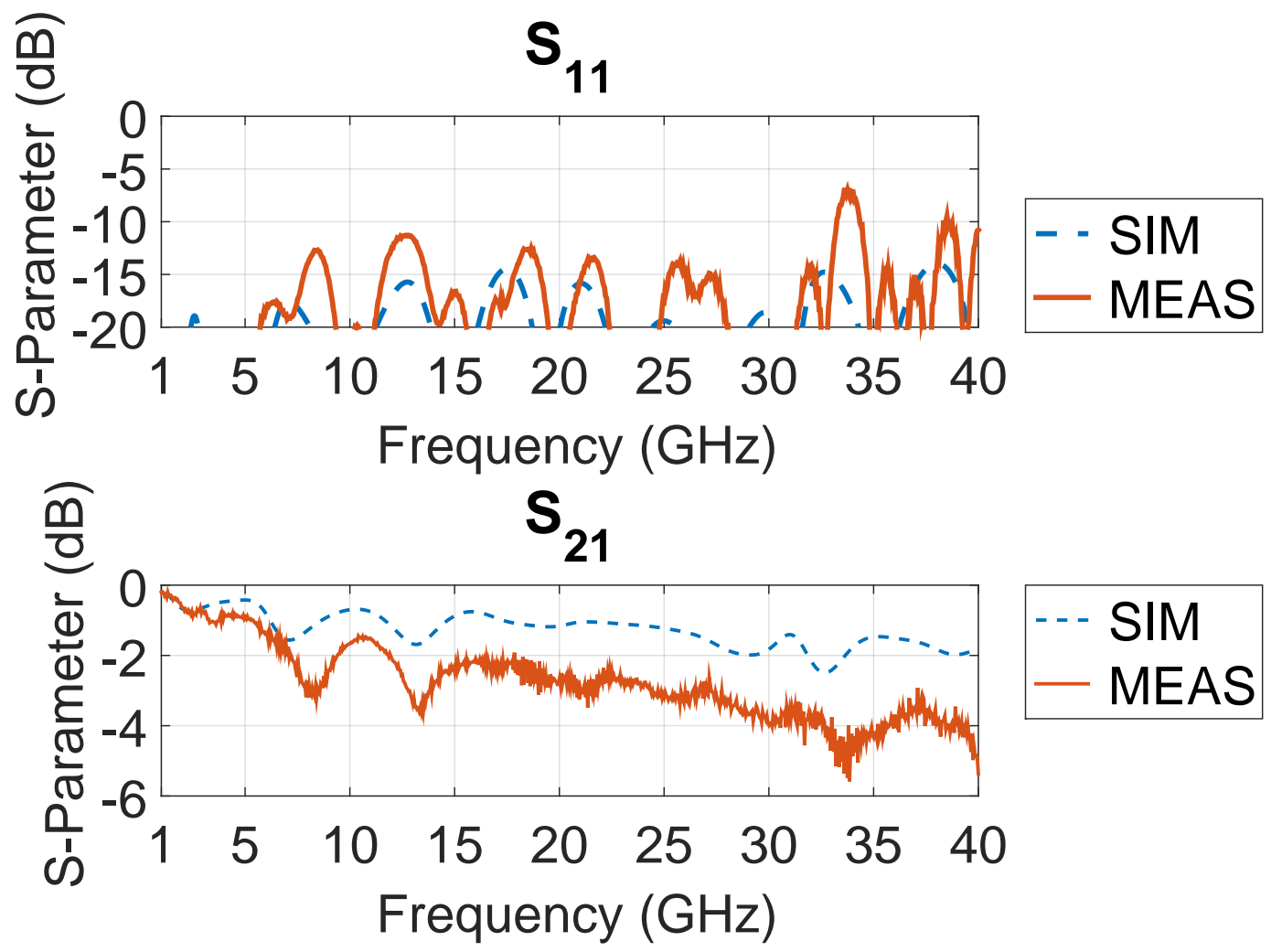

Figure 5.12: Simulated and measured S-parameters of the back-to-back PCB transition.

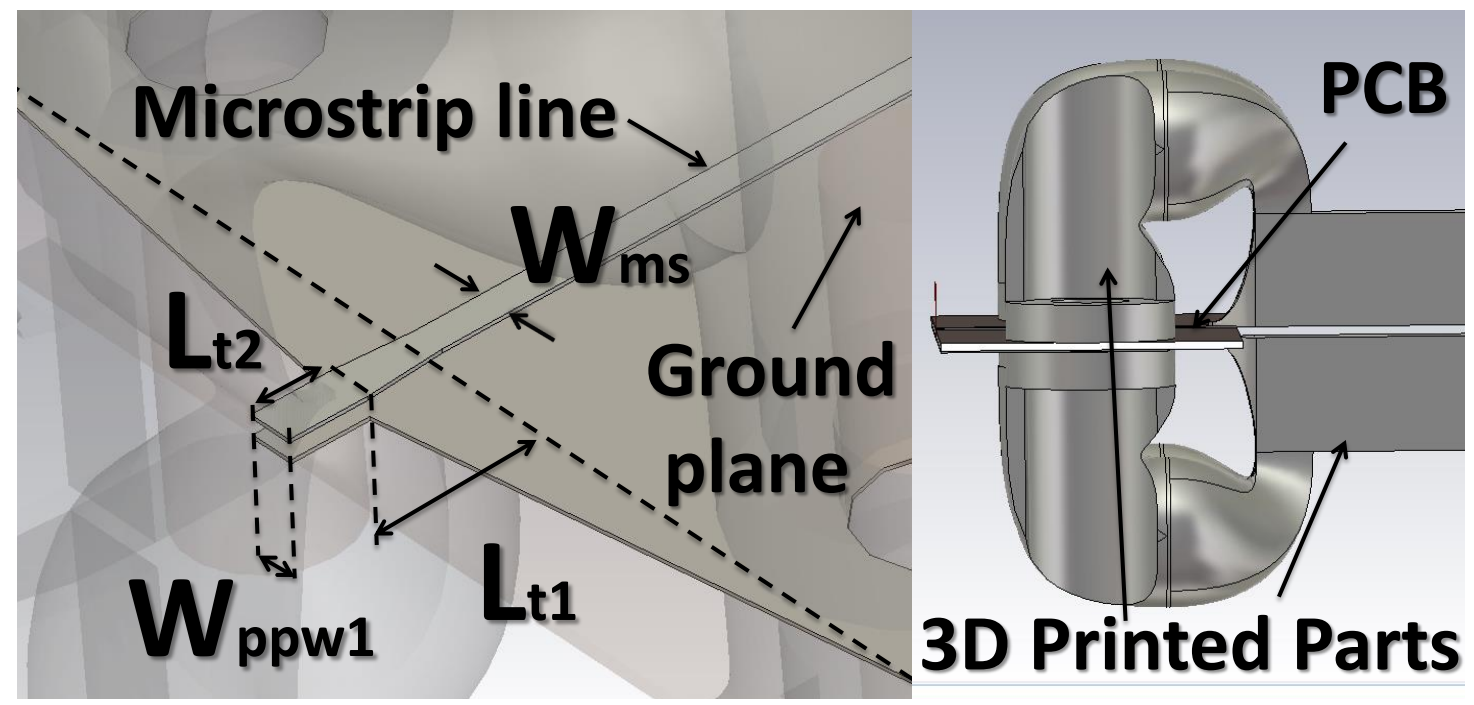

Figure 5.13: 3D sketch of the MSL-to-PPW transition on the PCB. A. Main geometrical parameters. B. General view. 


\subsection{A 3D Printed PCB integrated TEM Horn}

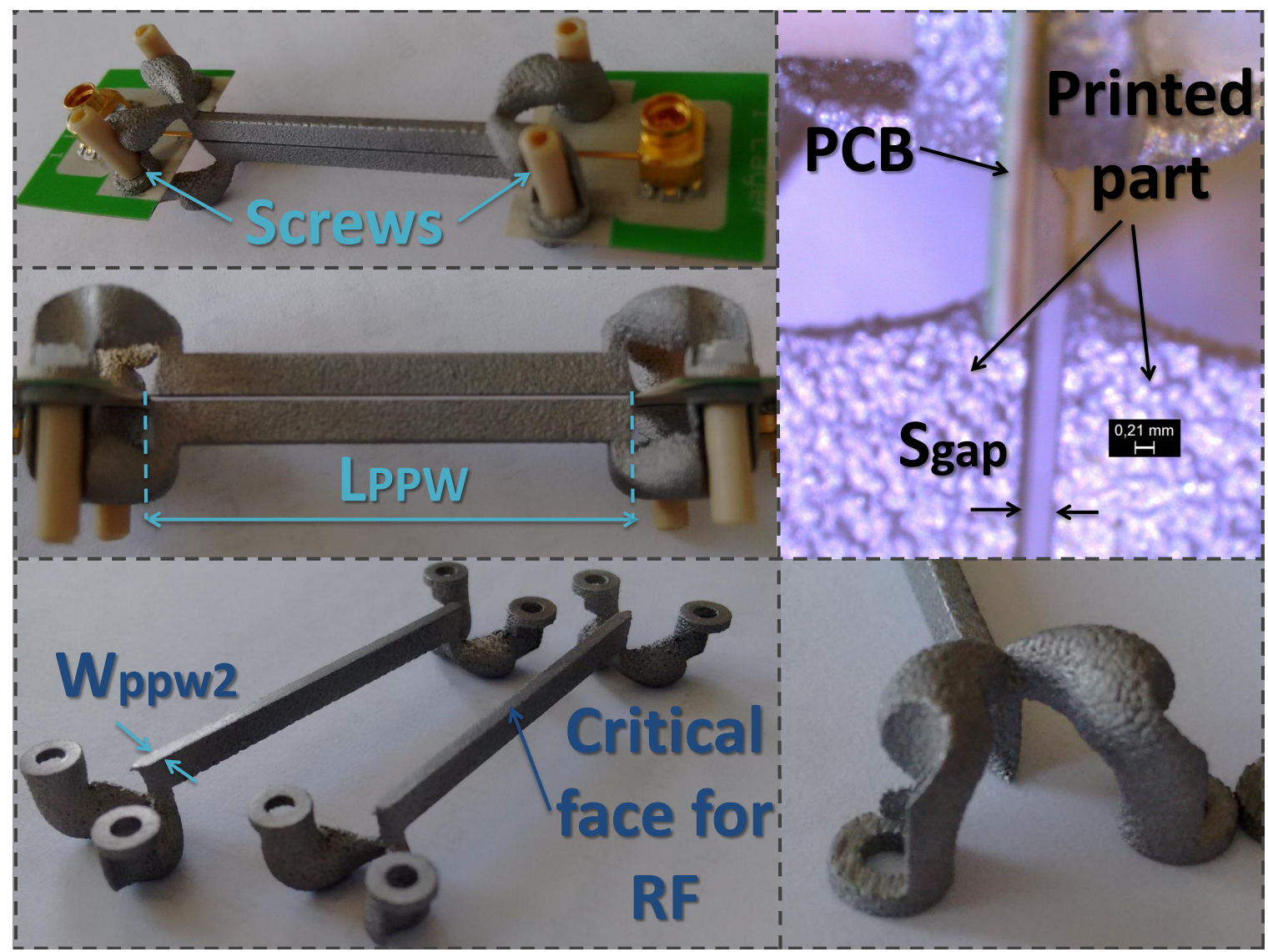

Figure 5.14: Picture of the back-to-back transition without $90^{\circ}$ bends. A. Screws. B. Sideways view showing $L_{p p w}$ and $w_{p p w 2}$. C. Unscrewed parts showing the critical face for RF. D. Microscope view showing $S_{\text {gap }}$. E. General view of the supporting legs.

measured with a microscope on the PCB to be $S_{\text {gap }}=0.30 \pm 0.01 \mathrm{~mm}$. In this case, the two faces which form the PPW, which are critical for the RF performance, are printed on top of a flat surface, therefore the measured accuracy is better than $\pm 100 \mu \mathrm{m}$.

The simulation and the measurement of the S-parameters present good agreement (see Fig. 5.15). The reflection is less than $-5 \mathrm{~dB}$ from $5-40 \mathrm{GHz}$. This is an acceptable value considering that the measured back-to-back transition has two transitions and its influence in the final antenna is approximately $6 \mathrm{~dB}$ less. The IL is a value between $-2 \mathrm{~dB}$ to -4 $\mathrm{dB}$ in the targeted frequency range. The metallic supporting legs generate resonances (see Fig. 5.16).

\section{Back-to-back 3D printed transition including $90^{\circ}$ bends}

Two $90^{\circ}$ bends are added to the previous transition as Fig. 5.17 depicts. As a consequence, the shape is more complex and it can not be printed on a flat surface. Thus, a worsening of the accuracy, especially in the bends, is expected. The air gap is measured within the straight section to be $S_{\text {gap } 1}=0.39 \pm 0.03 \mathrm{~mm}$, at its minimum to be $S_{g a p 2}=0.13 \mathrm{~mm}$ and 


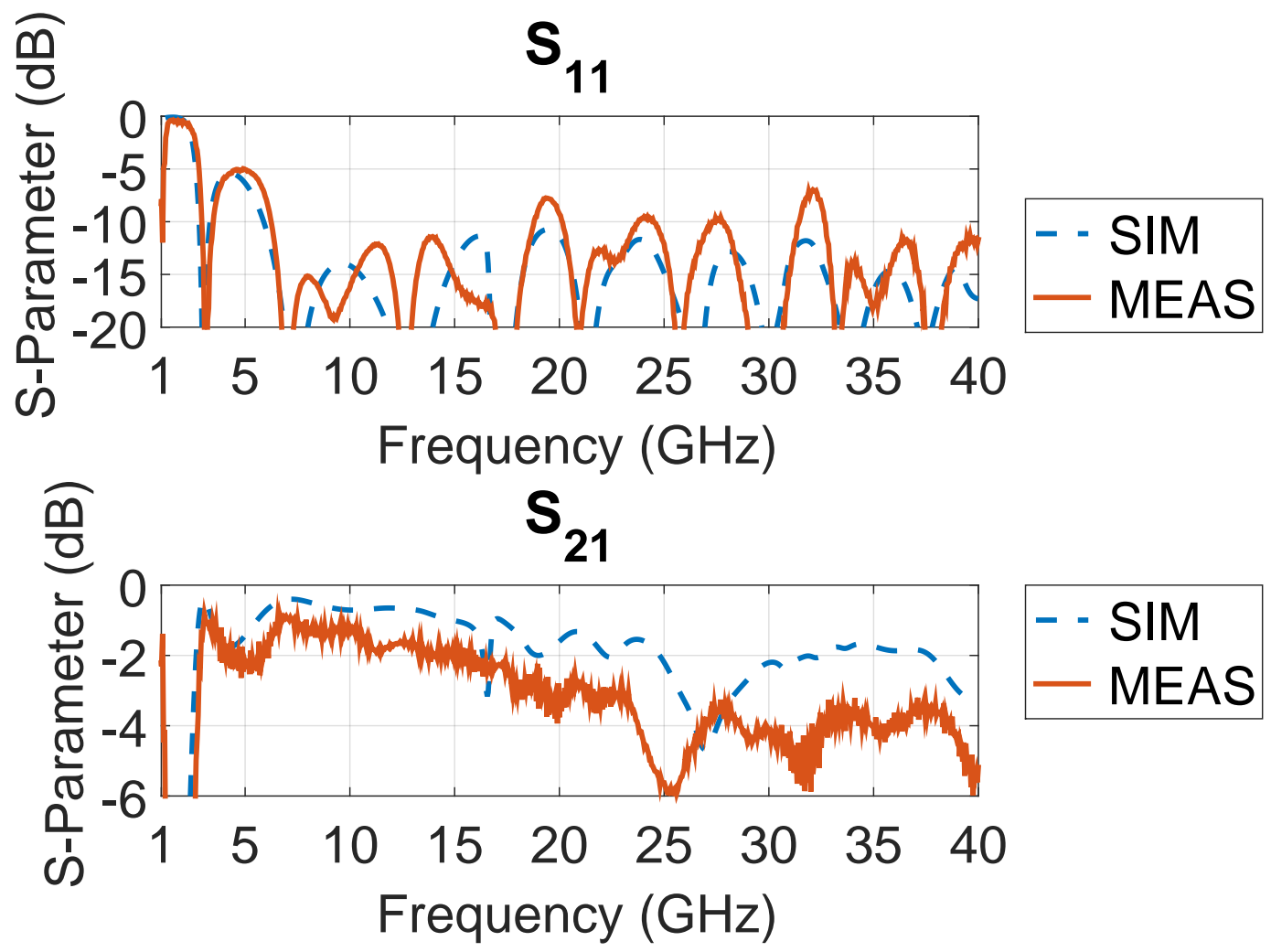

Figure 5.15: Simulated and measured S-parameters of the back-to-back transition without bends.

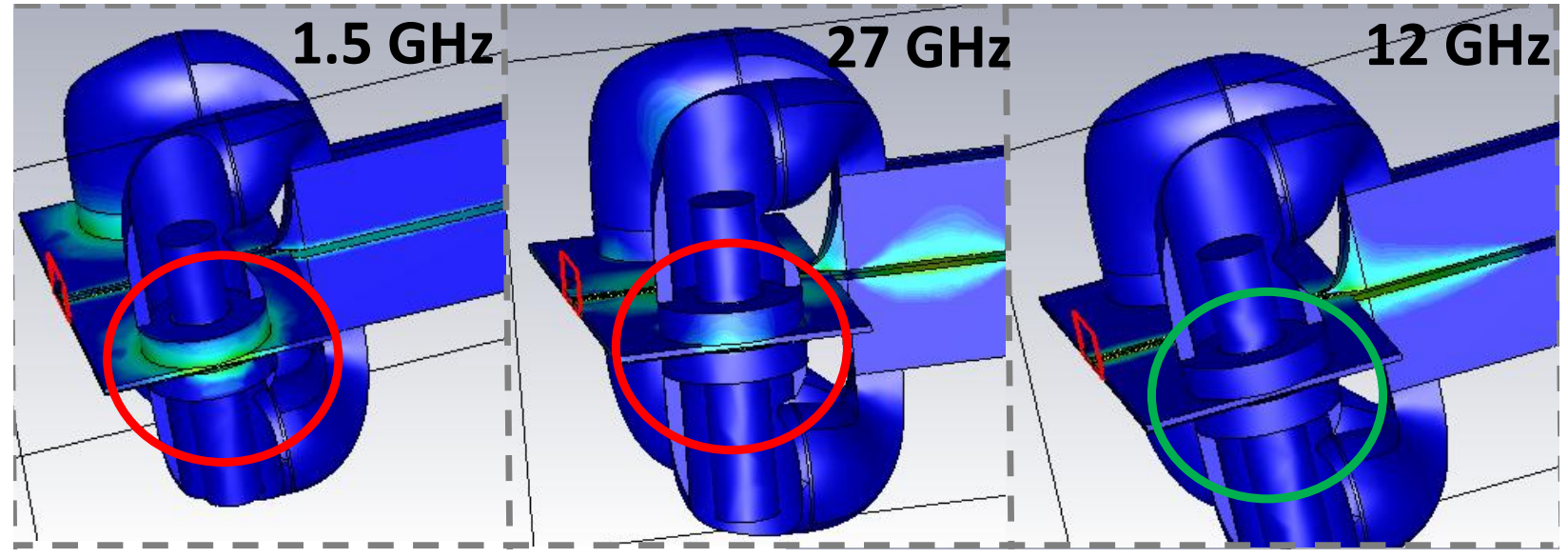

Figure 5.16: Simulated absolute value of the E-Field at $1.5 \mathrm{GHz}, 27 \mathrm{GHz}$ and 12 GHz. The red circles indicate the supporting legs which exite resonant modes at $1.5 \mathrm{GHz}$ and $27 \mathrm{GHz}$. The green circle indicates the supporting legs which do not excite resonant modes at $12 \mathrm{GHz}$. 


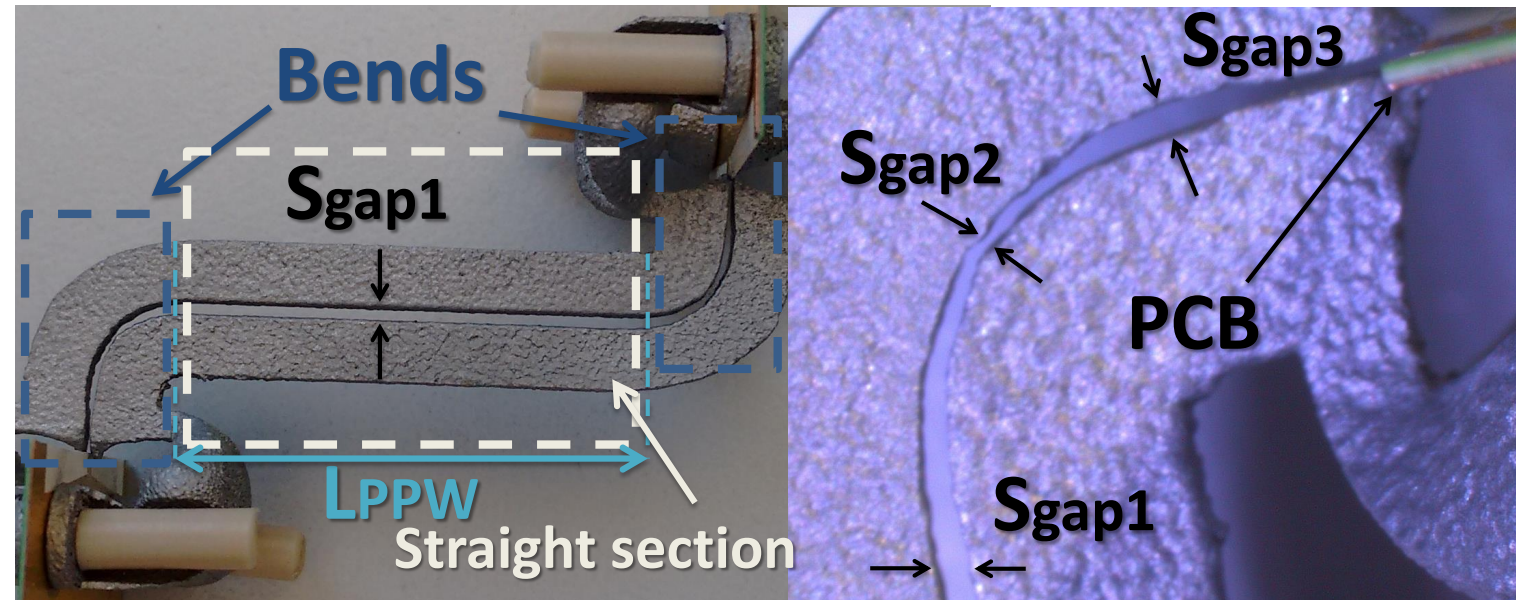

Figure 5.17: Back to back transition with $90^{\circ}$ bends. A. General view. B. Microscope view.

at its maximum to be $S_{\text {gap } 3}=0.46 \mathrm{~mm}$. Hence, impedance variations and higher roughness [31] exist.

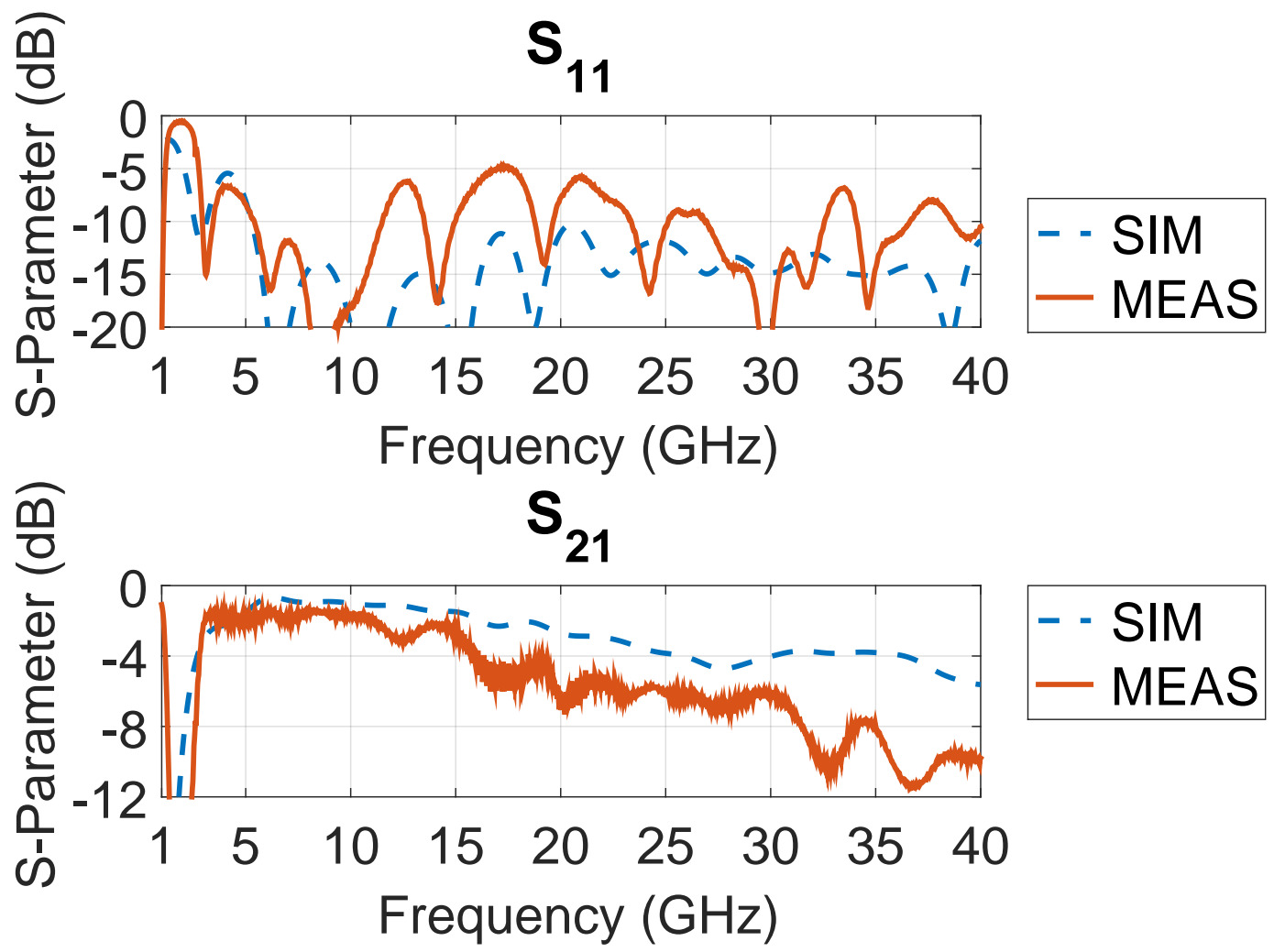

Figure 5.18: Simulated and measured S-parameters of the transition with bends. 
In general the measured S-parameters fit quite well with the simulation (see Fig. 5.18). However because of the mismatch introduced by fabrication tolerances, particularly in the bends, the RL deteriorates, especially between 13 and $22 \mathrm{GHz}$.

Nevertheless, the RL still presents a value below $-5 \mathrm{~dB}$ over the targeted frequency band. The IL is between -3 and $-10 \mathrm{~dB}$ from 5 to $40 \mathrm{GHz}$. It presents higher losses due to higher roughness in the entire structure and a longer length, since each bend is $7.58 \mathrm{~mm}$ long.

\subsubsection{Antenna Results}

A TEM horn antenna based on the transition and the $90^{\circ}$ bend presented above is designed following a published design procedure [32] (see Fig. 5.19). The aperture of the antenna is $42 \times 30 \mathrm{~mm}^{2}$ and its length is $L_{a}=85.5 \mathrm{~mm}$. The measured RL fits rather well the simulation (see Fig. 5.20). It is below $-10 \mathrm{~dB}$ for almost the whole targeted frequency range.

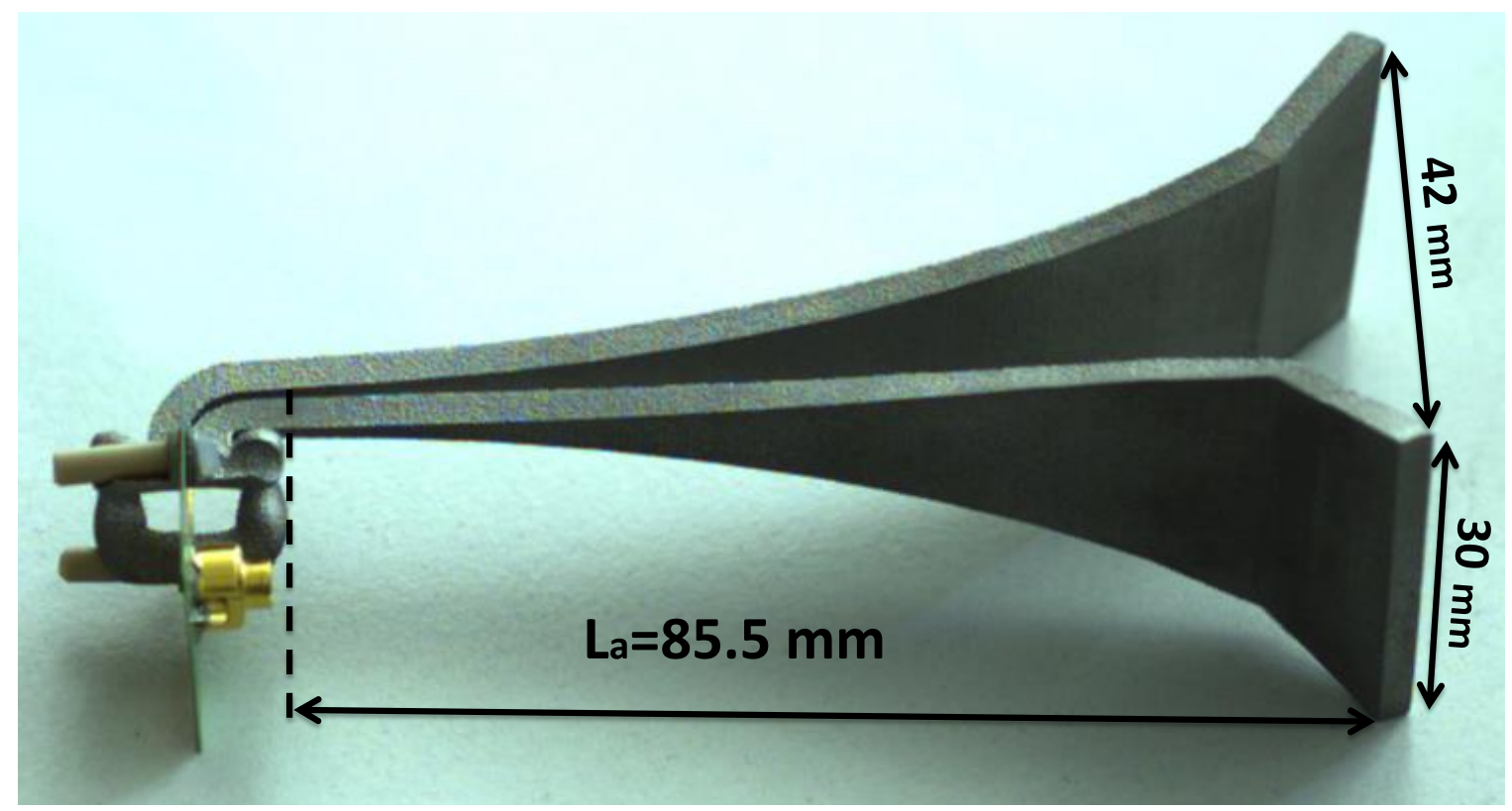

Figure 5.19: Photography of the final 3D Printed Antenna.

The roughness of the PPW and the fabrication tolerances explain the small differences between the measured and simulated RL.

An antenna without bend has been fabricated and measured in order to examine the effect of the bend in the gain (see Fig. 5.21). The antennas (notably the one without the $90^{\circ}$ bend) fit well with the simulation. The higher roughness and the higher inaccuracy in the PPW of the antenna with the $90^{\circ}$ bend lead to higher losses and thus, lower gain. 


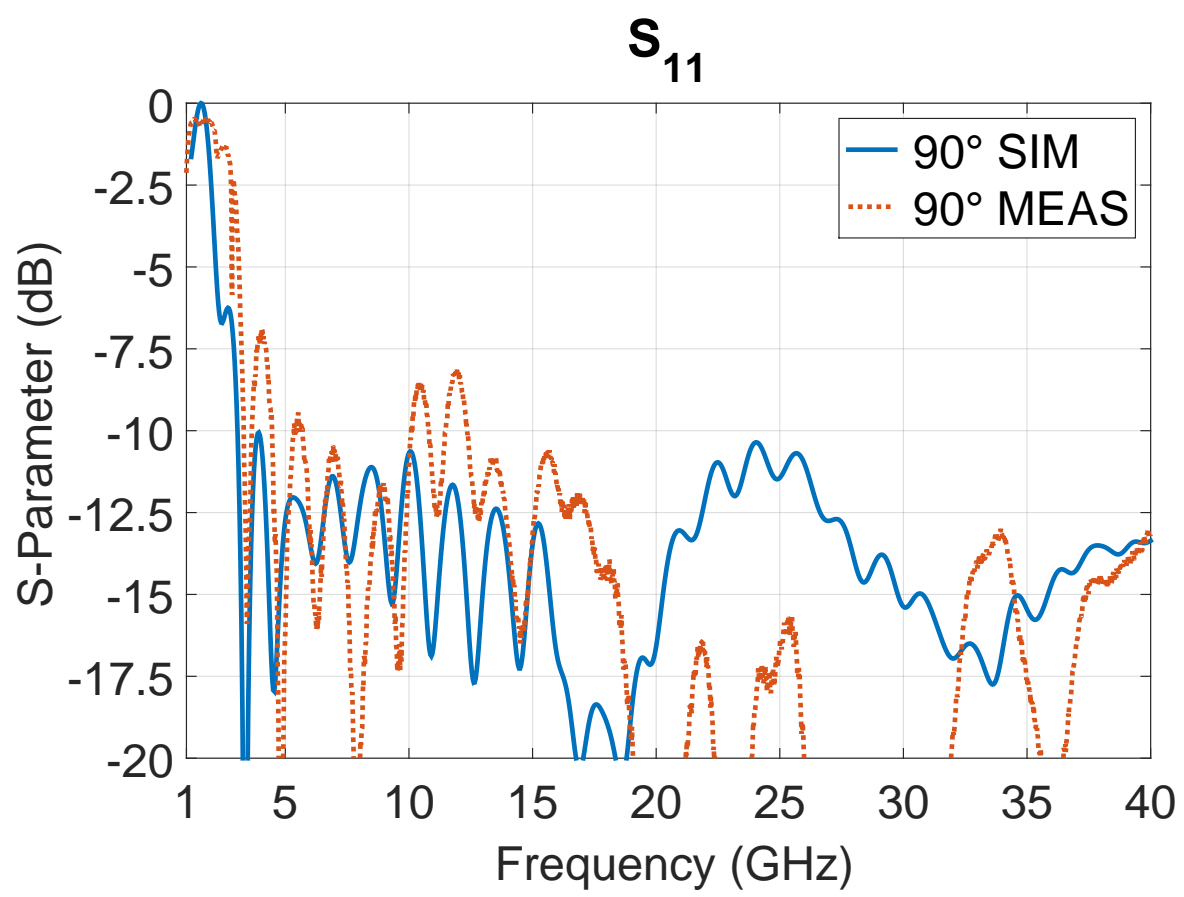

Figure 5.20: Simulated and measured $S_{11}$ of the antennas with $90^{\circ}$ bend.

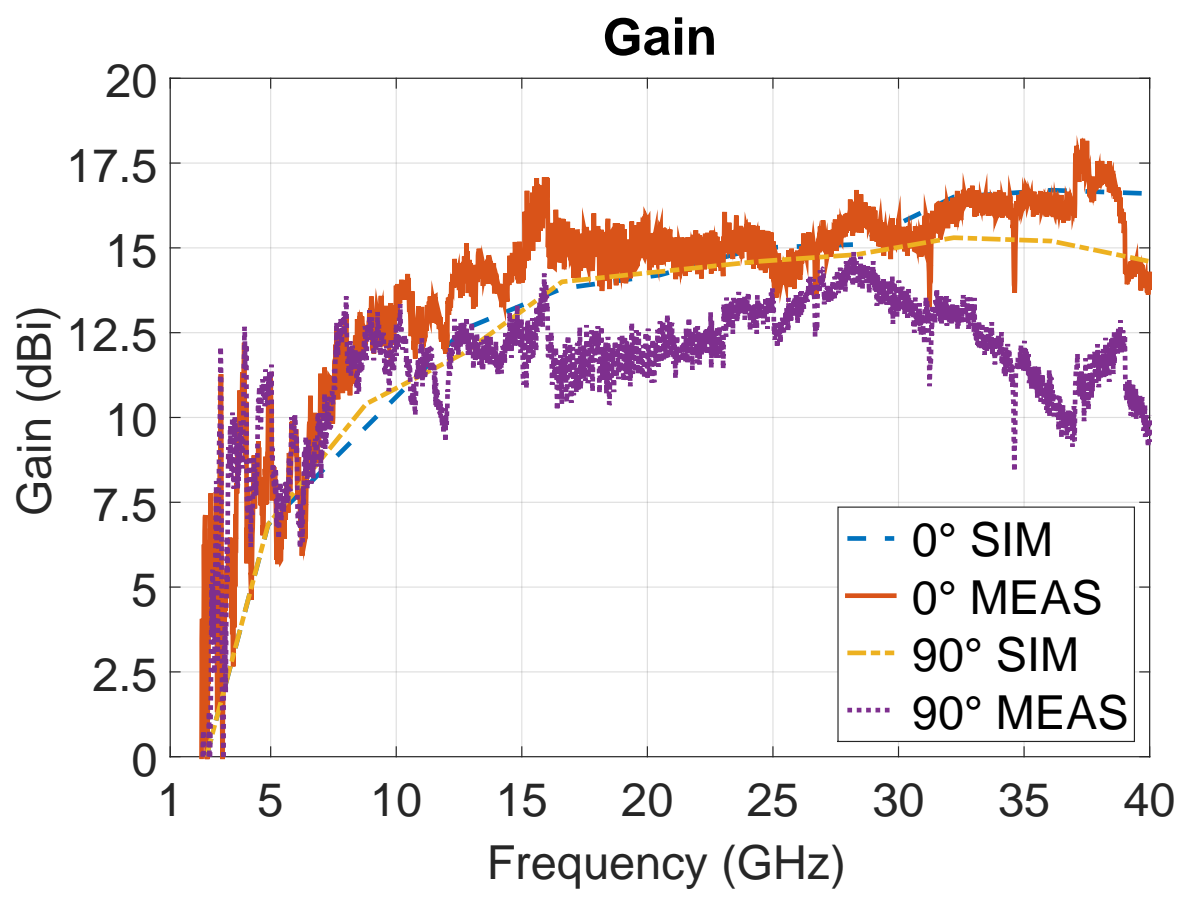

Figure 5.21: Simulated and measured gain of the antennas with and without bend. 


\subsubsection{Final Conclusions}

This chapter presents a novel antenna concept and a very broadband Wilkinson divider.

On the one hand, the novel 3D printed and integrable-with-PCB broadband antenna which radiates in the direction perpendicular to the PCB. The antenna and its transition have been measured with good results in terms of gain (approximatively $10 \mathrm{dbi}$ ) and RL (below -10 dB) over a wide frequency range (from 5 - $40 \mathrm{GHz}$ ). This results could be further improved by selecting a thicker substrate, in this manner, the fabrication tolerances would affect less the antenna performance. However, a too thick substrate would restrict the operational bandwidth of the antenna, since the cutoff frequency of high order modes in a microstrip line is inversely proportional to its height. In addition, the surface roughness can be reduced by using a surface finish treatment. This antenna could be also dielectric filled, in such a manner, the the 3D printed material can be partially metalized and act as a substrate including the whole system [5].

On the other hand, an analytical circuit model for a TWD is presented. It is used to determine a first set of $N$ (number of resistors), $R_{k}$ (value of resitance) and $L_{k}$ (position of resistors) parameters by an optimization. Then, a full wave simulation is run to consider additional effects and for comparison with the given specifications. At the end, a full wave optimization only with $L_{k}$ as parameter (minimizing the computational time by reducing the number of parameters) is launched. In order to validate the design method, a $2-38$ GHz TWD was fabricated and measured. To the best of the authors' knowledge this is the most wideband Wilkinson divider ever reported in scientific literature. 


\section{Bibliography}

[1] G.C. Tavik, C.L. Hilterbrick, J.B. Evins, J.J. Alter, J.G. Crnkovich, J.W. de Graaf, W. Habicht, G.P. Hrin, S.A. Lessin, D.C. Wu, and S.M. Hagewood, "The advanced multifunction RF concept", in IEEE Trans. Microw. Theory Techn.,, vol. 53, no. 3 pp. 1009-1020, Mar. 2005

[2] [Online]. Available: http://life.itu.int/radioclub/rr/art05.htm

[3] R. G. Wiley, "Electronic intelligence: The interception of radar signals", Artech House, 1985 - Technology \& Engineering - 284 pages

[4] M. Jankiramen, "Design of Multi-Frequency CW Radars", SciTech Publishing Inc, 2007 - 351 - ISBN-13:978-1-891121-56-2

[5] E. Miralles and V. Ziegler, "Circuit board for hf applications including an integrated broadband antenna", Publication number: US20170179607A1 (USA), DE102015225496A1 (GER) and EP3182504A1 (EU).

[6] E. Miralles, B. Schoenlinner, F.Ellinger and V. Ziegler "Fast Design Method and Validation of Very Wideband Tapered Wilkinson Divider" in Microwave Conference (EuMC), 2015 European, Paris, 2015, pp. 119-122.

[7] E.J. Wilkinson "An N-Way Hybrid Power Divider" in IEEE Trans. Microw. Theory Techn., Jan 1960 Vol. 8 No. 1 pp 116-118

[8] D.M. Pozar "Microwave Engineering" in Wiley 2012, ISBN 8126541903, 9788126541904

[9] C.T. Chiang and B.K. Chung "Ultra wideband power divider using tapered line," in Progress In electromagnetics Research, vol. 106, pp. 63-73, 2010.

[10] L. Chang, C. Liao, L. Chen. W.B. Lin, X. Cheng and Y.L. Wu, "Design of an ultrawideband power divider via the coarse-grained parallel micro-genetic algorithm," in Progress In electromagnetics Research, vol. 124, pp. 425-440, 2012.

[11] K. Shamaileh, M. Almalkawi, V. Devabhaktuni, N. Dib, B. Henin and A. Abbosh, "Non-uniform transmission line ultra-wideband wilkinson power divider," in Progress In electromagnetics Research, vol. 44, pp. 1-11, 2013. 
[12] R. Klopfenstein, A transmission line taper of improved design, in Proc. IEEE, vol. 44, no. 1, pp. 3135, Jan. 1956.

[13] E. Miralles, H. Esteban, C. Bachiller, A. Belenguer, and V. Boria, Improvement for the design equations for tapered microstrip-to-substrate integrated waveguide transitions, in IEEE Int. Conf. Electromagn. Adv. Appl. (ICEAA), Torino, Italy, Sep. 2011.

[14] H. Wheeler, Transmission lines with exponential taper, Proc. IEEE, vol. 27, no. 1, pp. 6571, Jan. 1939.

[15] Mattias Björkman and Kenneth Holmstrom "Global optimization using DIRECT algorithm in matlab" in Adv. Model. Optim., 1999, vol. 1, pp. 17-37.

[16] I.J. Petrick and T. W. Simpson "3D printing disrupts manufacturing: how economies of one create new rules of competition." Research-Technology Management, 2013 Taylor \& Francis.

[17] J.Zuniga, D.Katsavelis, J.Peck, J.Stollberg, M.Petrykowski, A.Carson and C. Fernandez "Cyborg beast: a low-cost 3d-printed prosthetic hand for children with upper-limb differences." BMC Research Notes 2014.

[18] [Online]. Available: https://localmotors.com/3d-printed-car/

[19] [Online]. Available: https://3dprint.com/tag/3d-printed-house/

[20] M.Tomlin, J.Meyer "Topology optimization of an additive layer manufactured (ALM) aerospace part." in The 7th Altair Technology Conference 2011.

[21] M.Liang, C.Shemelya, E. MacDonald, R.Wicker and H.Xin "3-D Printed Microwave Patch Antenna via Fused Deposition Method and Ultrasonic Wire Mesh Embedding Technique" in IEEE Antennas and Wireless Propag. Lett., vol. 14, pp.1346-1349 2015.

[22] M.Liang, X. Yu, C.Shemelya, E. MacDonald and H.Xin "3D Printed Multilayer MSL Structure with Vertical Transition toward Integrated Systems" in 2015 IEEE MTT-S International Microwave Symposium, Phoenix, AZ, 2015, pp. 1-4 .

[23] C.Shemelya, M.Zemba, M.Liang, D. Espalin, C.Kief, H.Xin, R.B. Wicker and E.W. MacDonald "3D PRINTING MULTI-FUNCTIONALITY. Embedded RF Antennas and Components" in 2015 9th European Conference on Antennas and Propagation (EuCAP), Lisbon, 2015, pp. 1-5 .

[24] P. Nayeri, M. Liang, R. A. Sabory-Garcia, M. Tuo, F. Yang, M. Gehm, H.Xin, A. Z. Elsherbeni "3D Printed Dielectric Reflectarrays: Low-Cost High-Gain Antennas at Sub-Millimeter Waves" in IEEE Transactions on Antennas and Propagation, vol. 62, no. 4, pp. 2000-2008, April 2014. 
[25] C.R. Garcia, R.C. Rumpf, H.H. Tsang and J.H. Barton "Effects of extreme surface roughness on 3D printed horn antenna" in ELECTRONICS LETTERS 6th June 2013 Vol. 49 No. 12.

[26] F. Gianesello, A. Bisognin, D. Titz, C. Luxey, C.A. Fernandes, J.R. Costa and D. Gloria "3D printing technology: Enabling innovative \& cost effective industrial antenna solution" in 2016 International Workshop on Antenna Technology (iWAT), Cocoa Beach, FL, 2016, pp. 55-56.

[27] G. Tavik, C. Hilterbrick, J. Evins, J. Alter, J. Crnkovich, J. de Graaf, I. Habicht, G. P. Hrin, S. Lessin, D. Wu, and S. Hagewood, "The advanced multifunction rf concept" IEEE Trans. Microw. Theory Techn., vol. 53, no. 3, pp. 10091020, Mar. 2005.

[28] E. Miralles, T. Multerer, A. Ganis, B. Schoenlinner, U. Prechtel, A. Meusling, J. Mietzner, C. Weckerle, H. Esteban, M. Vossiek, M. Loghi, and V. Ziegler, "Multiductional and Compact 3D FMCW MIMO Radar System with Rectangular Array for Medium Range Applications" IEEE Aerospace and Electronic Systems Magazine. Accepted.

[29] A. Ahmed, Y. Zhang, D. Burns, D. Huston, and T. Xia "Design of UWB Antenna for Air-Coupled Impulse Ground-Penetrating Radar" in IEEE Geoscience and Remote Sensing Letters, vol. 13, no. 1, pp. 92-96, Sep. 2016.

[30] H.A. Wheeler "Transmission-Line Properties of Parallel Strips Separated by a Dielectric Sheet" in IEEE Transactions on Microwave Theory and Techniques, vol. 13, no. 2, pp. 172-185, Mar 1965.

[31] C. L. Holloway and E. F. Kuester "Power loss associated with conducting and superconducting rough interfaces," in IEEE Transactions on Microwave Theory and Techniques, vol. 48, no. 10, pp. 1601-1610, Oct 2000

[32] J.A.G. Malherbe "Hybrid Elliptic TEM Horn with Symmetric Main Beam" in Antenna Technology and Applied Electromagnetics (ANTEM), 2012 15th International Symposium on, Toulouse, FR, 2012, pp. 1-4. 


\section{Chapter 6}

\section{ESIW for MIMO Radar applications}

Traditionally, waveguide technology has been used for high performance applications despite of its bulkiness. Moreover, planar microwave circuits, which can be integrated on well-known Printed Circuit Boards (PCBs) as microstrip lines, are very compact but not that efficient. Recently, a new technology called Empty Substrate Integrated Waveguide (ESIW) [1], which can be integrated in PCBs and joins advantages of planar circuits (low cost and integration with ICs) and waveguides (low losses), was developed. By removing the inner dielectric and replacing the posts with metallic walls, this technology increases the performance of SIW circuits in terms of losses. Some key RF components have been fabricated in this technology [2]-5]. In this chapter, a very highly efficient ESIW slotted waveguide antenna [6] and a cross-guide coupler[7], which are suitable for a MIMO Radar application [8], are presented.

\subsection{ESIW Slotted Antenna}

In this section, a design strategy for a slotted ESIW antenna (see Fig. 6.1) with operational frequency from 16 to $16.5 \mathrm{GHz}$ is presented. ESIW technology posses high efficiency (if compared to SIW) and low cost in terms of design time. ESIW structures replace the characteristic via row of SIW devices with a conductive wall. This fact enables the possibility to utilize a full-wave simulator with very low computational cost.

\subsubsection{Waveguide Selection}

First of all, a proper rectangular waveguide in which the operational frequency band (from 16 to $16.5 \mathrm{GHz}$ ) is within its monomode range must be selected. The cut-off frequency for the $\mathrm{TE}_{10}$ mode is:

$$
f_{1 c u t-o f f}=\frac{c}{2 a}
$$

where, $c$ is the speed of light and $a$ the longer side of the aperture of a waveguide. The first higher order mode limits the modomode range. Considering $b<a / 2$ (due to its flat 


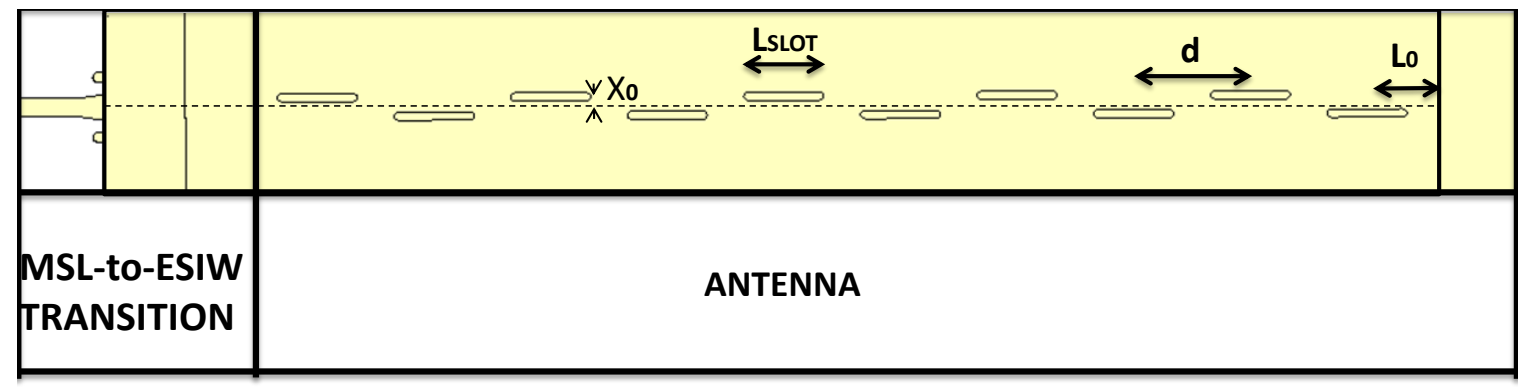

Figure 6.1: Layout of the designed antena with its main parameters.

profile), this mode is the $\mathrm{TE}_{20}$ and its cut-off frequency is:

$$
f_{2 c u t-o f f}=\frac{c}{a}
$$

Considering the commented constraints the design parameters $a$ and $b$ are set to be $15.8 \mathrm{~mm}$ and $1.033 \mathrm{~mm}$, respectively. In such a manner, $f_{1 \text { cut-off }} \approx 9.5 \mathrm{GHz}$ and $f_{2 c u t-o f f} \approx 19 \mathrm{GHz}$.

\section{CROSS-SECTION}

\section{VIEW}

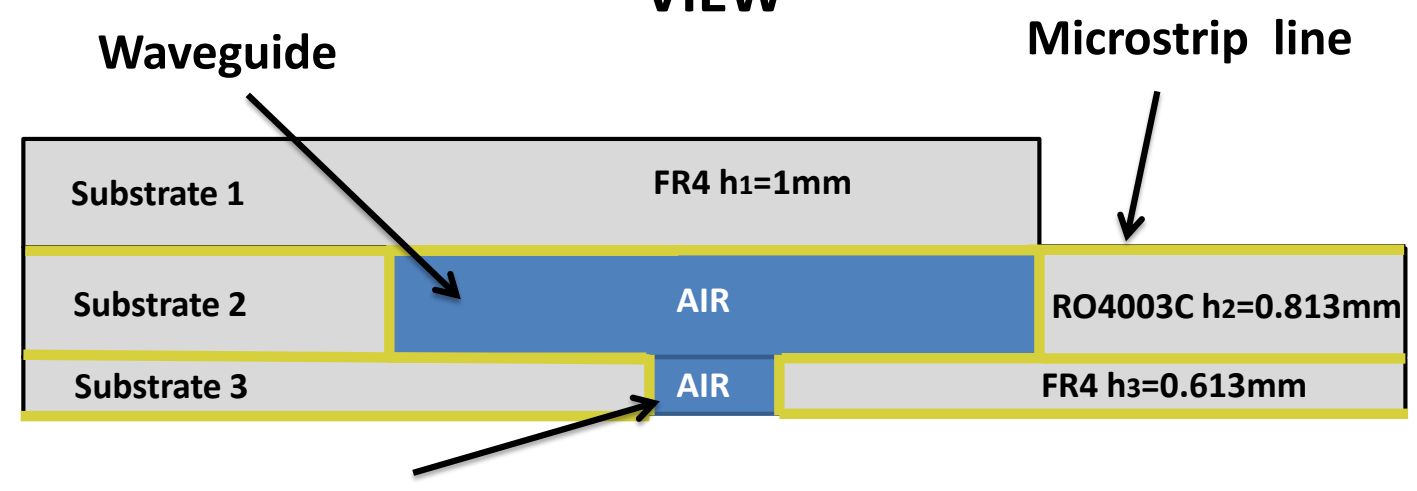

\section{Slots}

Figure 6.2: Cross section view of the selected layer stack-up.

\subsubsection{Layer Stack-up selection}

Fig. 6.2 illustrates the cross section of the proposed device. It consists of three substrates. Substrate 1 is an FR4 substrate and has a height $\left(h_{1}\right)$ of $1 \mathrm{~mm}$. Substrate 2 is a RO4003C substrate, has a height $\left(h_{2}\right)$ of $0.813 \mathrm{~mm}$ and serves as microstrip line substrate. It provides 
the desired height $b$ to the waveguide, considering $b=h_{2}+2 t$ (the thickness of the metalization is $\mathrm{t}=0.11 \mathrm{~mm})$. The substrate 3 is an FR4 and has a height $\left(h_{3}\right)$ of $0.613 \mathrm{~mm}$. The slots are drilled through the substrate 3 .

\subsubsection{D full-wave Simulation}

The commercial finite element simulator CST has been used in all the simulations. The presented antenna is designed by following a two step process.

First of all, a simulation of the antenna without the transition is performed. This simulation is based on ideal lossless waveguide, since the dielectric substrate included in the transition is not considered, and the metallic parts are modeled as perfect electric conductors. This leads to a simulation with a very low computational cost. Fig. 6.1 illustrates the main parameters of the device. The distance between the shortcircuit and the fist slot $\left(L_{0}\right)$ must be set in such a manner that the first slot is illuminated with a maximum E-field. This is achieved with the following value:

$$
L_{0}=\frac{\lambda_{g}}{4}
$$

The slots must be fed with maxima of E-field and with the same phase. As a consequence, the distance between slots is fixed to:

$$
d=\frac{\lambda_{g}}{2}
$$

The rest of the design parameters (length of the slots $L_{S L O T}$ and offset is $x_{0}$ ) are optimized using a simplex algorithm until the desired return loss at the operational frequency is achieved. Table 6.1 presents the value of the design parameters of the fabricated antenna.

In the last step of the design process, the structure is integrated with a more complex and higher computational effort model, which includes the losses due to the conductor $(\sigma=$ $\left.5.8 \cdot 10^{7} \mathrm{~S} / \mathrm{m}\right)$ and the losses due to the dielectric substrate $\left(\epsilon_{r}=3.55\right.$ and $\left.\tan (\delta)=0.0027\right)$. This model is also including a previously presented transition from microstrip to ESIW [1].

Table 6.1: Design parameters of the fabricated device.

\begin{tabular}{ccccc} 
Parameter & $L_{0}$ & $\mathrm{~d}$ & $L_{S L O T}$ & $x_{0}$ \\
\hline Value & 5.68 & 11.36 & 7.80 & 0.91 \\
\hline
\end{tabular}

\subsubsection{Measured Results}

A prototype of the designed structure has been manufactured and measured in order to validate the simulation. The device is fabricated by emptying a hole in a substrate. After that, this substrate is metalized, in such a manner that the lateral walls of the slotted waveguide are created. Later, the microstrip-to-ESIW taper and the bottom slots are cut. Then, the bottom layer is metalized. Fig. 6.3 A illustrates these three layers. Finally, top 


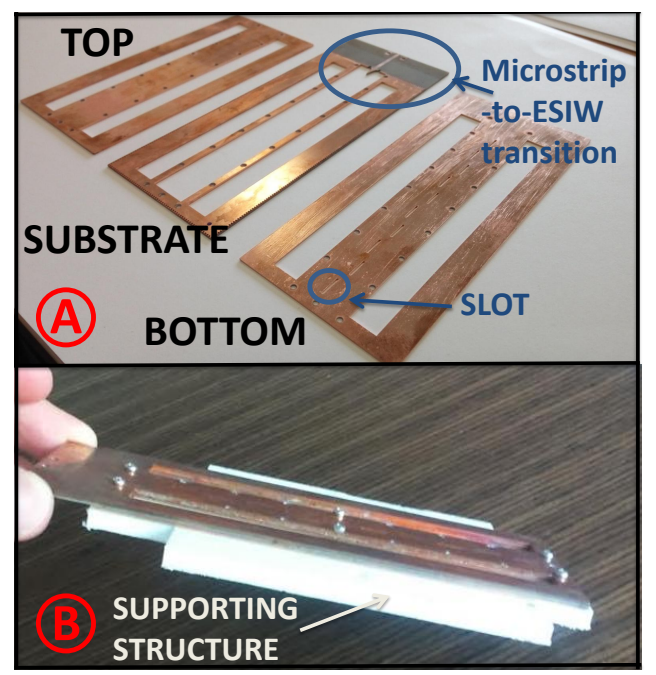

Figure 6.3: A. Separated layers of the fabricated device. B. Stacked layers of the fabricated device.

and bottom layers are soldered to the substrate, in such a way that the final result can be observed in Fig. 6.3 B. In order to manufacture the proposed device an LPKF Protomat S103 circuit board plotter was used. This machine manufactures with an accuracy of $0.15 \mathrm{~mm}$ and repeatability of $\pm 0.001 \mathrm{~mm}$. The LPKF Mini contac RS system was used for the electroplating.

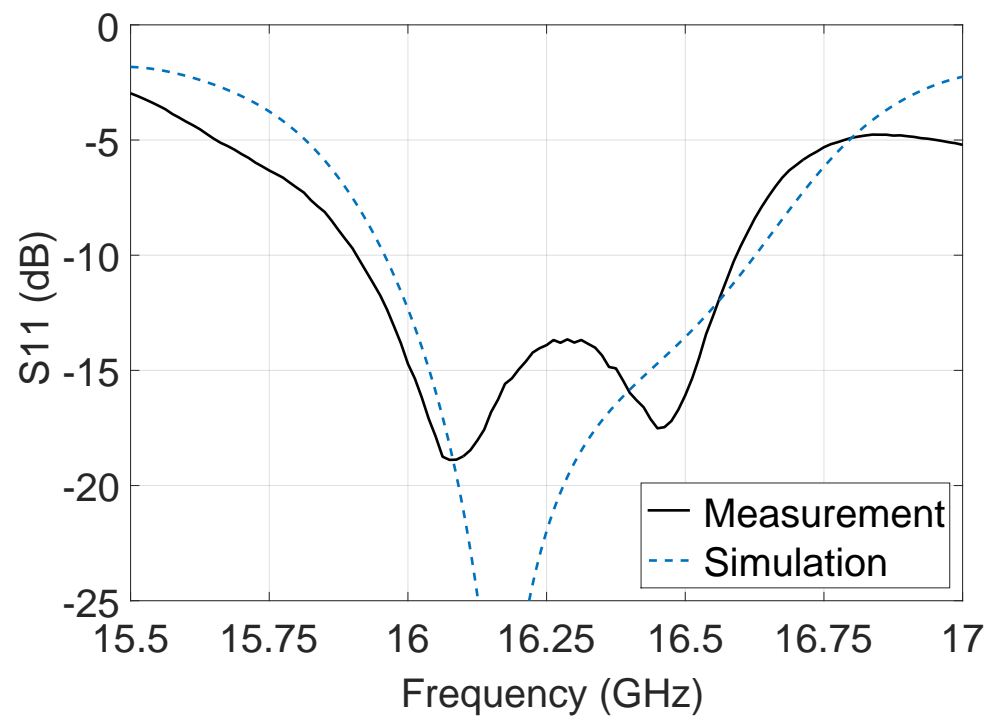

Figure 6.4: Measurement of the fabricated Antenna.

Fig. 6.4 presents a comparison between the simulated and measured reflection coefficient. Both measured and simulated $S_{11}$ are below $-13.5 \mathrm{~dB}$ in the operational frequency band (16 to $16.5 \mathrm{GHz}$ ). The small differences between simulation and measurement are due 
to manufacturing process effects related to substrate permittivity variations, imperfections during the metalization and soldering, and milling errors.

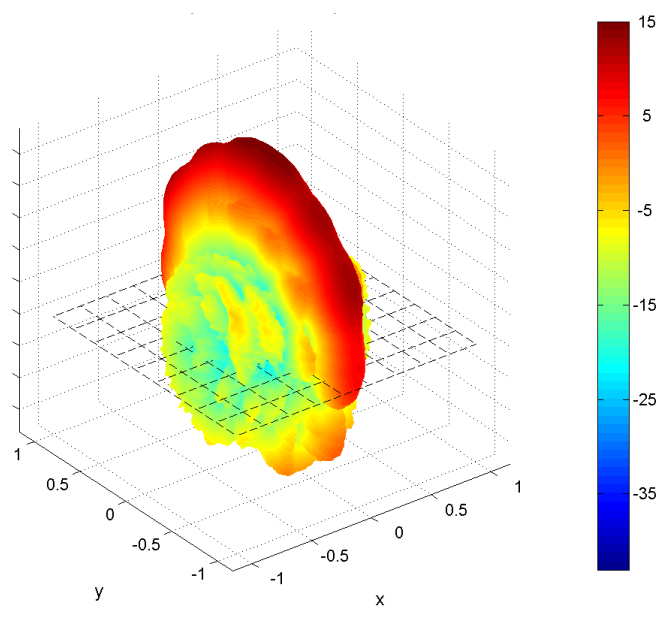

Figure 6.5: Measured 3D gain.

The measured 3D gain at the central frequency of the operational frequency band $(16.25 \mathrm{GHz})$ is illustrated in Fig. 6.5. The maximum of radiation is obtained along the z-axis. The measured radiation pattern looks like a classical slotted waveguide antenna. The measurement is in good agreement with the simulation, considering that the simulated gain is $15.8 \mathrm{dBi}$ and that the measured gain is $15 \mathrm{dBi}$.

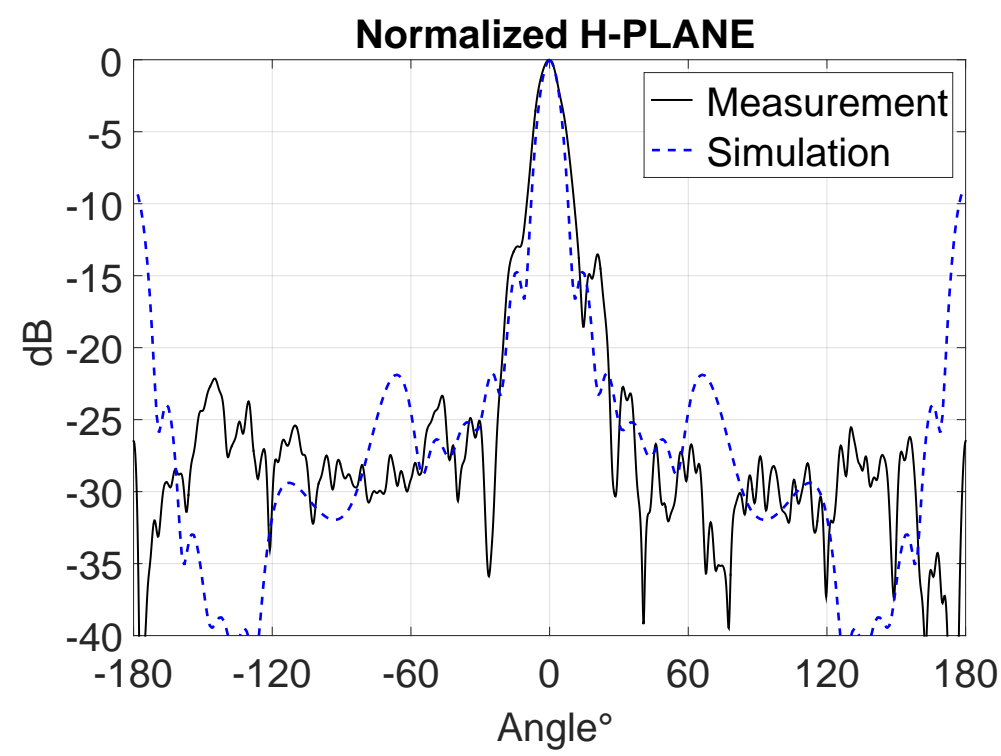

Figure 6.6: Comparison between simulation and measurement of the H-plane radiation pattern of the fabricated antenna. 


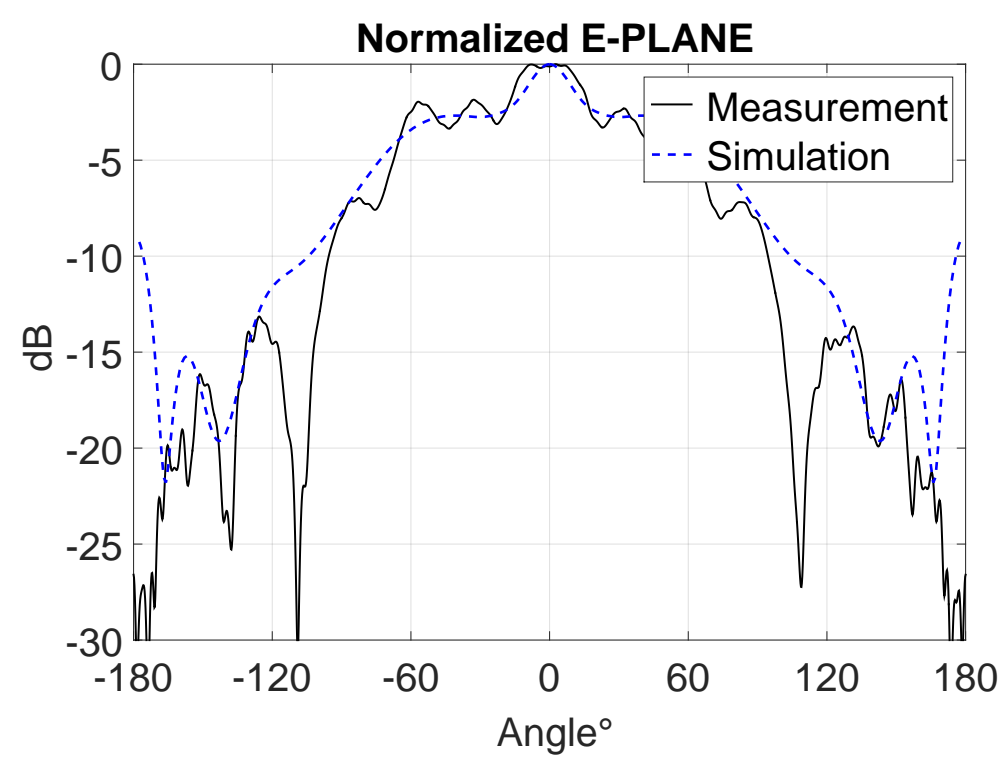

Figure 6.7: Comparison between simulation and measurement of the E-plane radiation pattern of the fabricated antenna.

Fig. 6.6 and Fig. 6.7 illustrate a comparison between the simulation and measurement of the H-plane and the E-plane radiation patterns, respectively. The measurement fits very well the simulation. Nevertheless, there are some small discrepancies due to fabrication inaccuracies, which lead to an increment of the ripple in the radiation pattern and a worsening of the Side Lobe Level (SLL).

Table 6.2: Summary of radiation efficiencies

\begin{tabular}{cc} 
Antenna Configuration & Efficiency \\
\hline Simulated ESIW & $96.83 \%$ \\
Simulated SIW & $72.61 \%$ \\
Measured ESIW & $90.78 \%$
\end{tabular}

Table 6.2 introduces a summary of radiation efficiencies. This table shows that the simulated radiation efficiency of a slotted ESIW antenna including its transition to a $50 \Omega$ the microstrip line is $96.83 \%$, while the simulated radiation efficiency for the same antenna, designed in SIW technology, is $72.61 \%$. The measured radiation efficiency of the fabricated ESIW antenna is $90.78 \%$. The measured ESIW radiation efficiency is superior to the simulated SIW radiation efficiency. 


\subsection{Cross-guide Moreno directional coupler in empty substrate integrated waveguide}

This section introduces a fabricated cross-guide coupler in planar form. The coupler consists of two crossed waveguides, which are connected through two coupling slots placed on its common wall. The presented device can be integrated within a complete MIMO Radar with more components, as filters, circulators, antennas or even IC in the same Printed Circuit Board (PCB) reducing significantly the whole size of the system.

\subsubsection{Cross-guide Coupler}

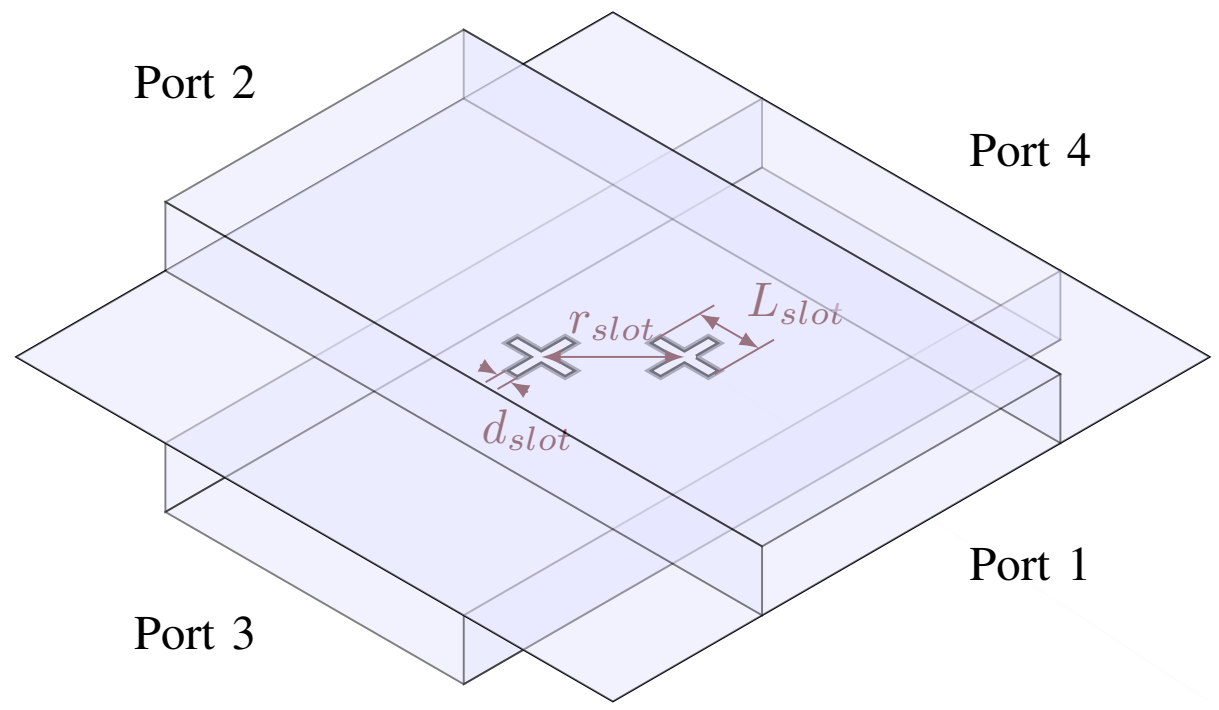

Figure 6.8: Representation of a waveguide Cross-guide coupler.

Fig. 6.8 shows a Cross-guide coupler in a rectangular waveguide. The four ports have been numbered. A small fraction of the signal traveling from port 1 to port 2 (main waveguide) passes through the cross-shaped coupling slots to the secondary waveguide and is directed to port number 3. Port 2 is the direct port. Port 3 is the coupled port. And port 4 is the isolated port. Thus, $\left|S_{31}\right|$ is the coupling, which should be small so that it does not distort the main flow and relatively constant over the desired frequency range, and $\left|S_{32}\right|=\left|S_{41}\right|$ is the isolation, which should be smaller than the coupling. The difference between $\left|S_{32}\right|$ and $\left|S_{31}\right|$ is the directivity. If the directivity is small, then undesired reflections coming from port 2 could pass to port 3 and mask the fraction of signal coming from port 1 and coupled to port 3 . 


\section{CROSS-SECTION}

\section{VIEW}

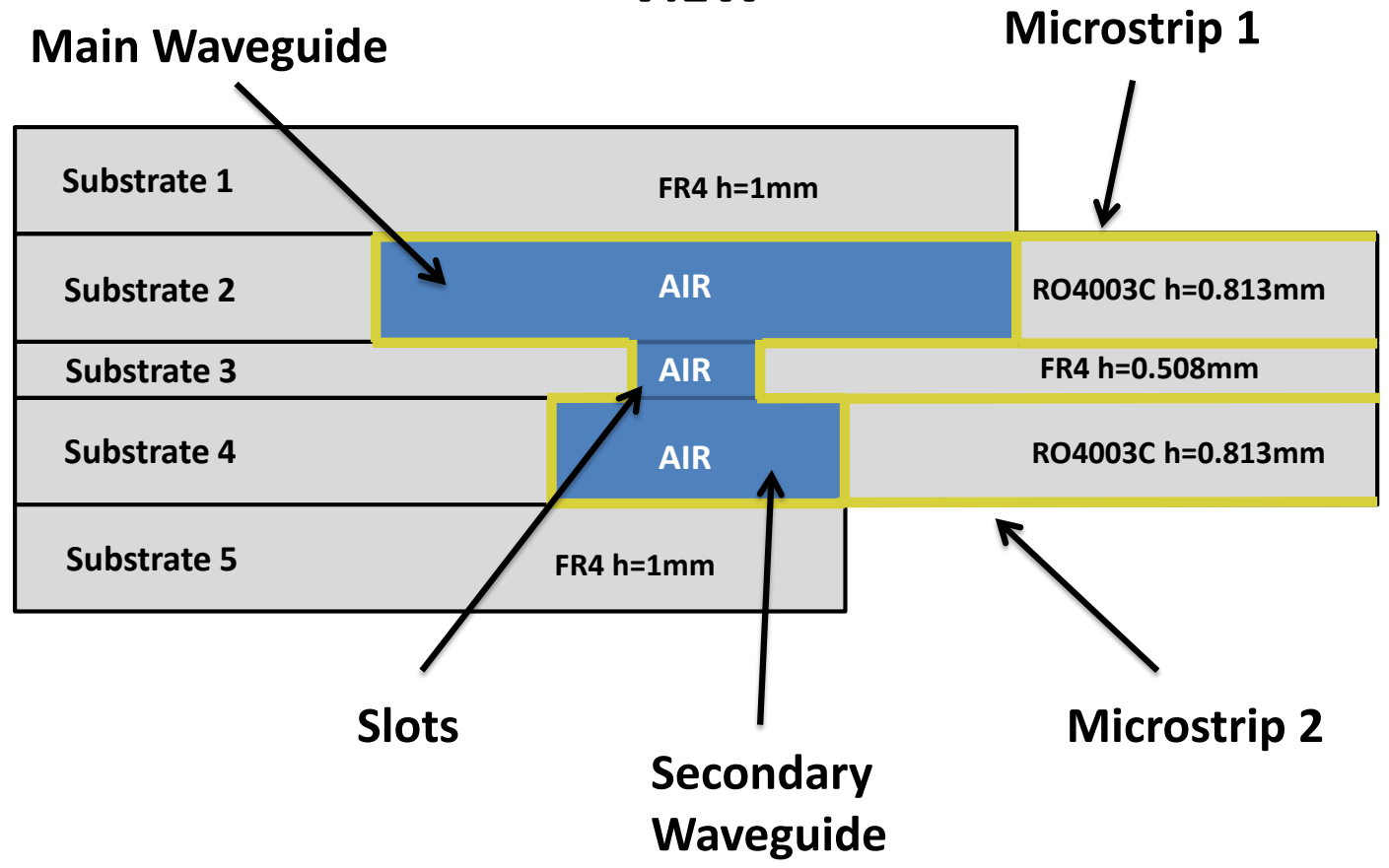

Figure 6.9: Layer stackup of the designed PCB

\subsubsection{Implementation in ESIW}

Fig. 6.9 depicts the five different substrate layers needed to manufacture a Cross-guide coupler in ESIW. All layers are manufactured with standard planar circuit manufacturing techniques as described in [9].Fig. 6.10 introduces the fabricated subtrate layers, the layers are numbered corresponding to the substrate layers of Fig. 6.9. Layers 2 and 4 (main and secondary ESIW lines) have been manufactured in this work using a RO4003C substrate (height $h=0.813 \mathrm{~mm}$, relative electric permittivity $\epsilon_{r}=3.55$, metal thickness $\left.m_{1}=0.0525 \mathrm{~mm}\right)$, so the height of the ESIW is $b=h+2 m_{1}=0.918 \mathrm{~mm}$. The width of the ESIW has been chosen to fit for the WR62 $(a=15.7988 \mathrm{~mm})$. The transitions from microstrip line to ESIW are presented in [9].

Layer 3 contains the coupling slots. The thickness of this substrate has been chosen to be the thinnest available in our laboratory $(0.5080 \mathrm{~mm})$. The total thickness of the coupling layer is $t=0.508+2 m_{1}=0.613 \mathrm{~mm}$. From [10] we know that the smaller the $d_{\text {slot }}$, the greater the directivity. As a consequence, the smallest drill of our laboratory has been used $\left(d_{\text {drill }}=1 \mathrm{~mm}\right)$. Therefore, after its metalization $d_{\text {slot }}=d_{\text {drill }}-2 m_{2}=0.965 \mathrm{~mm}$ (the metalization on the wall is $m_{2}=0.0175 \mathrm{~mm}$ ). Finally, all substrates are integrated 


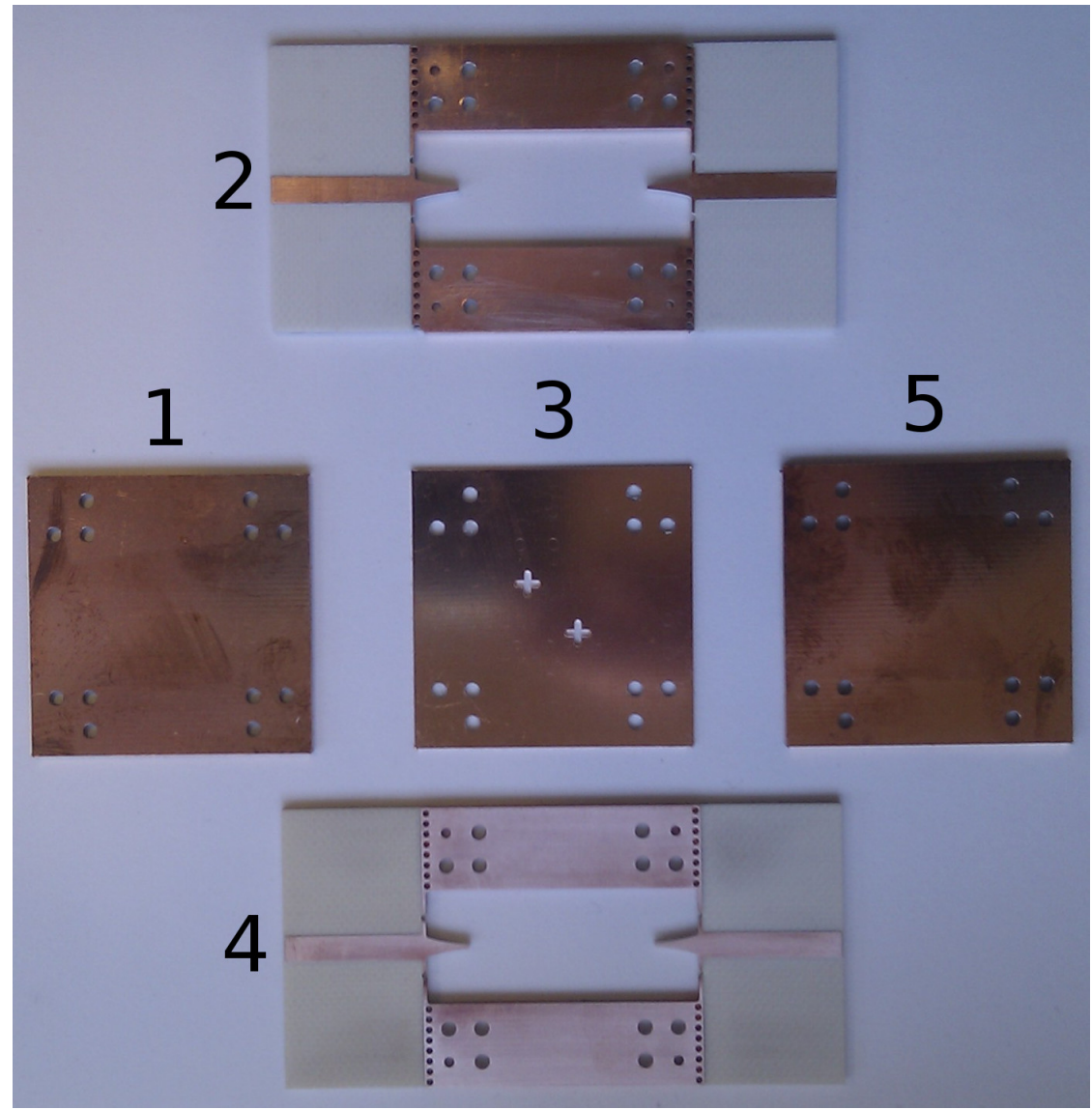

Figure 6.10: Photography of the separated subtrate layers. The layers are numbered corresponding to the substrate layers of Fig. 6.9

together, as Fig. 6.11 illustrates.

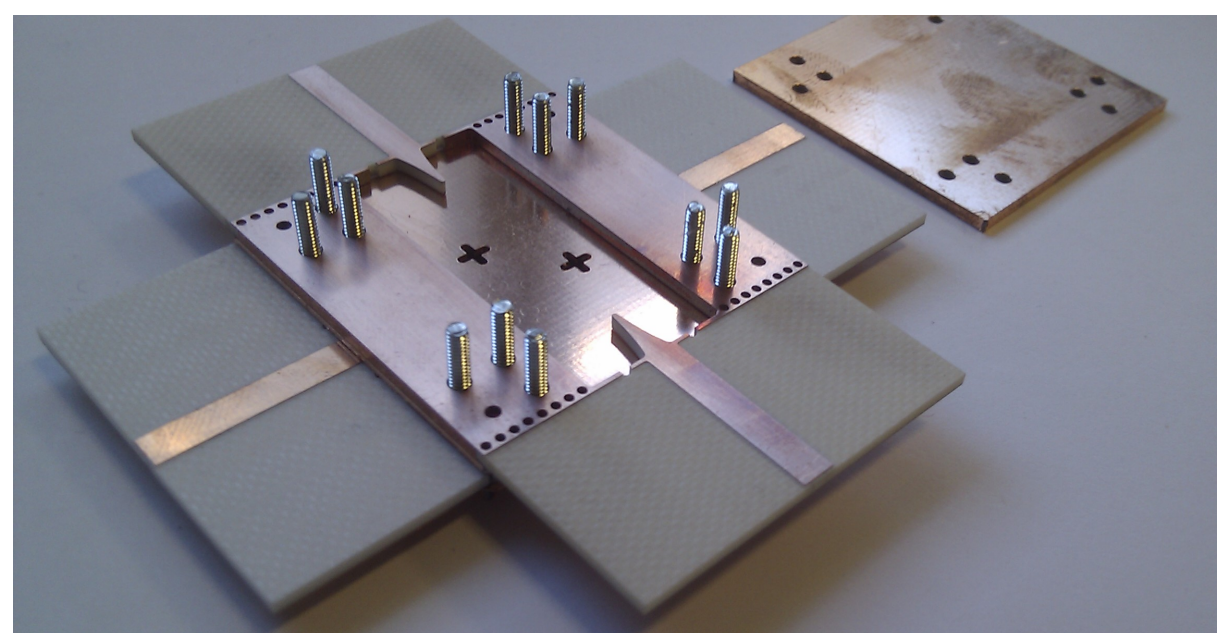

Figure 6.11: Photography of the mounted device. 
All the layers have been drilled with alignment holes, so that the different layers are piled using alignment screws, as can be seen in Fig. 6.11. All the layers are soldered together with soldering paste and the screws are then removed.

\subsubsection{Specifications And Design}

Table 6.3 lists the desired specifications for our design. These specifications are based on typical specifications of similar couplers for space applications in waveguide technology. The design parameters of the Cross-guide coupler are the dimensions of the cross-shaped coupling slots shown in Fig. $6.8\left(d_{\text {slot }}, r_{\text {slot }}\right.$ and $\left.L_{\text {slot }}\right) . d_{\text {slot }}$ has already been fixed by using the smallest available drill of our laboratory.

Table 6.3: Specifications of the designed coupler

\begin{tabular}{cccc}
\hline Frequency & Coupling & Directivity & Return Loss \\
\hline $12.4 \mathrm{GHz}-18 \mathrm{GHz}$ & $20.5 \mathrm{~dB} \pm 1$ & $15 \mathrm{~dB}$ & $20 \mathrm{~dB}$ \\
\hline
\end{tabular}

A similar procedure as the one described in a previous article [11] was used. This time, a two iteration process was followed in order to find out the best values for $r_{\text {slot }}=3.45 \mathrm{~mm}$ and $L_{\text {slot }}=4.93 \mathrm{~mm}$. In the first iteration a simplified model in a commercial full wave simulator, which is based on an ordinary lossless waveguide structure, was simulated. After that a fine tune adding loses and the microstrip to ESIW transitions was performed.

\subsubsection{Results}

A two port network analyzer and two matched loads have been used to measure the Sparameter of the Device Under Test (DUT). In order to subtract the effect of the coaxial to microstrip transitions from the measurements, a Thru-Reflect-Line (TRL) calibration procedure has been used, so that these measurements only include the ESIW coupler plus the transitions from ESIW to microstrip.

As Fig. 6.12 depicts, the insertion loss is around $1 \mathrm{~dB}$ (including microstrip to ESIW transitions) and the reflection (see Fig. 6.13) is lower than $-20 \mathrm{~dB}$ over the whole bandwidth. Moreover, Fig. 6.14 illustrates the coupling, which is between $19.5 \mathrm{~dB}$ and $21.5 \mathrm{~dB}$ over the targeted frequency range. The Isolation (see Fig. 6.15), which is lower than -37 dB from 12 to $18 \mathrm{GHz}$. Furthermore, the directivity is presented in Fig. 6.16, it is greater than the targeted $15 \mathrm{~dB}$. In general terms the simulation and the measurements fit rather well. As a conclusion, the measured results meet the specifications from table 6.3 over the whole frequency range of a WR62 waveguide.

Table 6.4 shows a comparison of broadband cross-guide couplers in planar form. The presented device improves the performance in terms of directivity of [13]. [12] presents 
6.2 Cross-guide Moreno directional coupler in empty substrate integrated waveguide

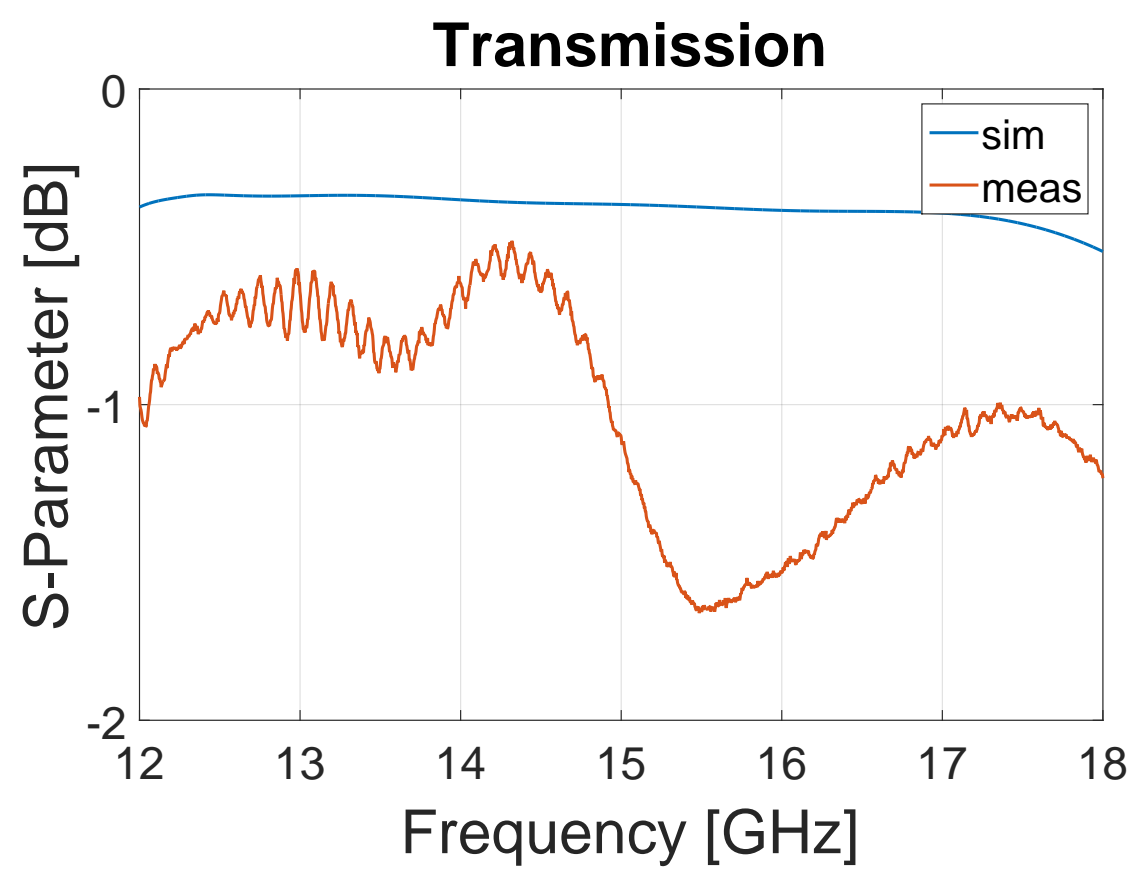

Figure 6.12: Comparison between measured and simulated Transmission $\left(\left|S_{21}\right|\right)$.

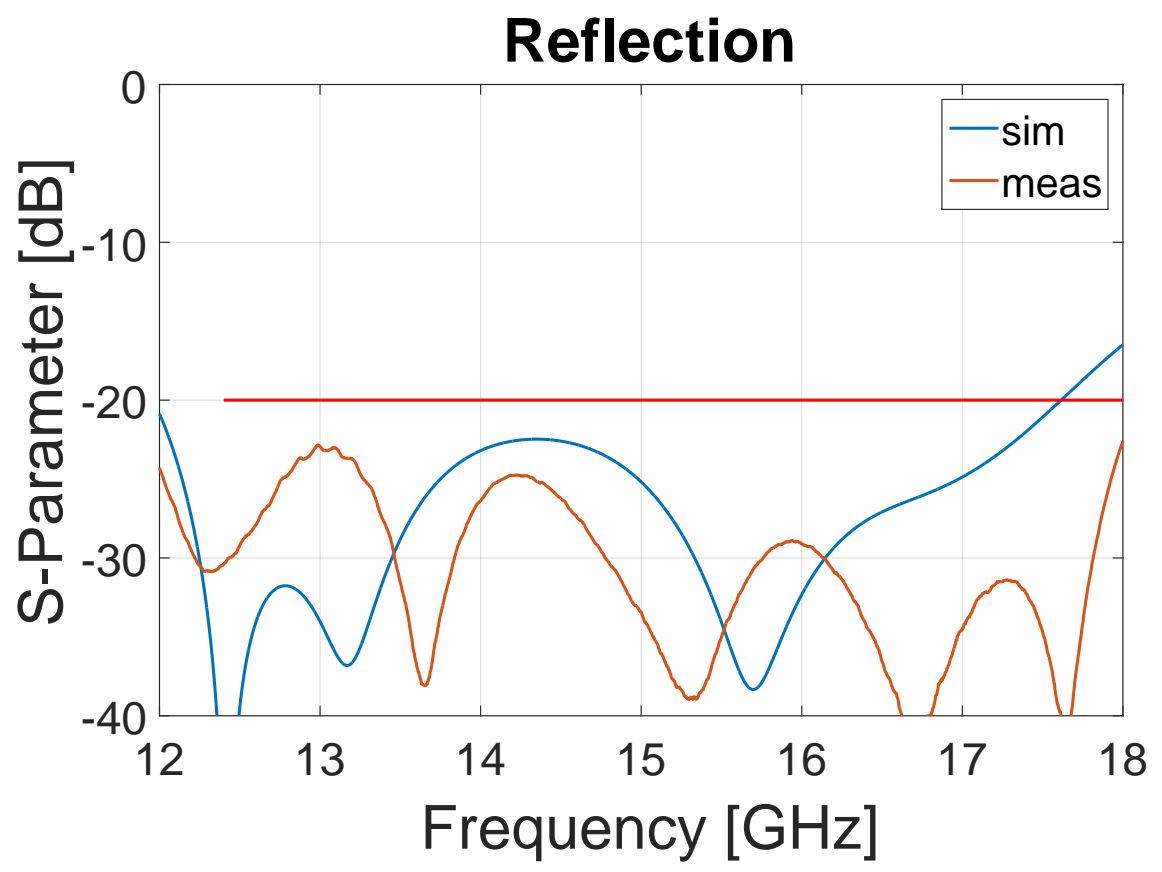

Figure 6.13: Comparison between measured and simulated Return loss $\left(\left|S_{11}\right|\right)$. 


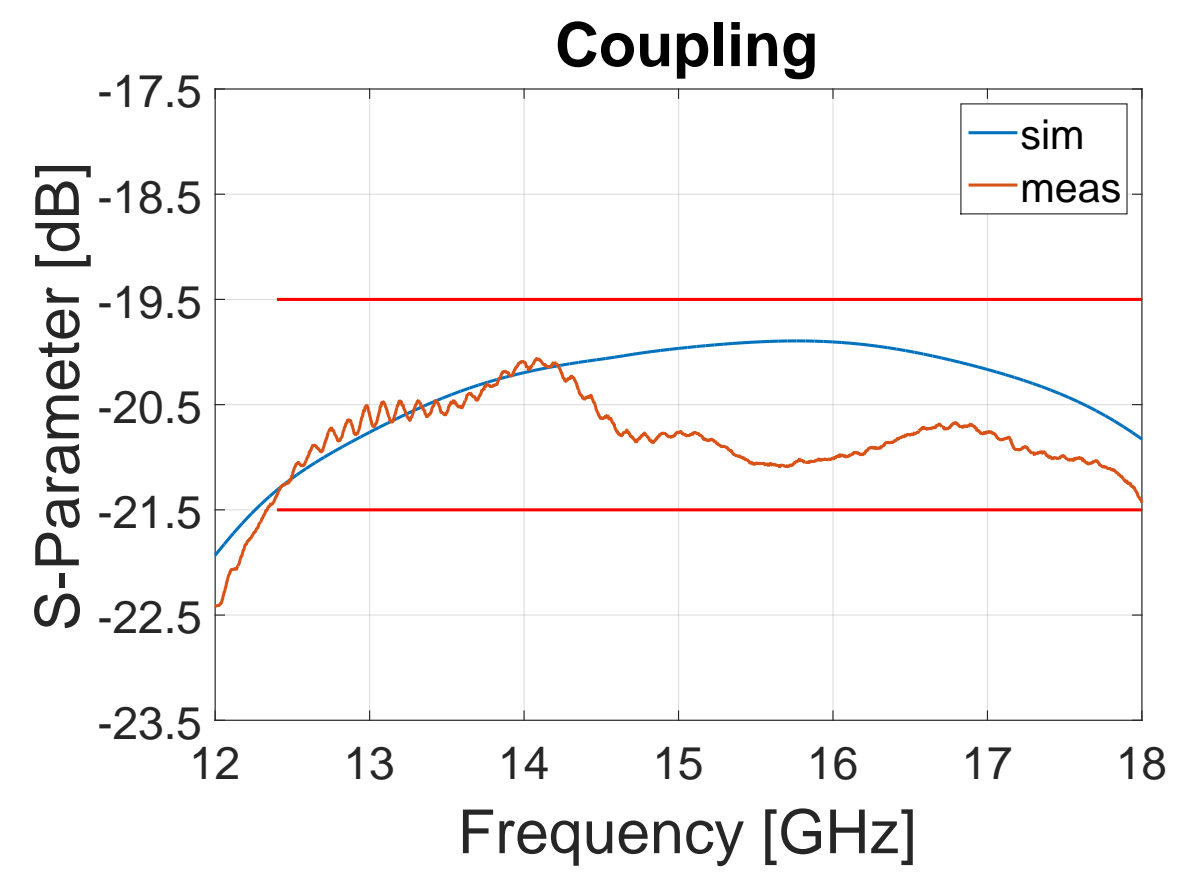

Figure 6.14: Comparison between measured and simulated Coupling $\left(\left|S_{31}\right|\right)$.

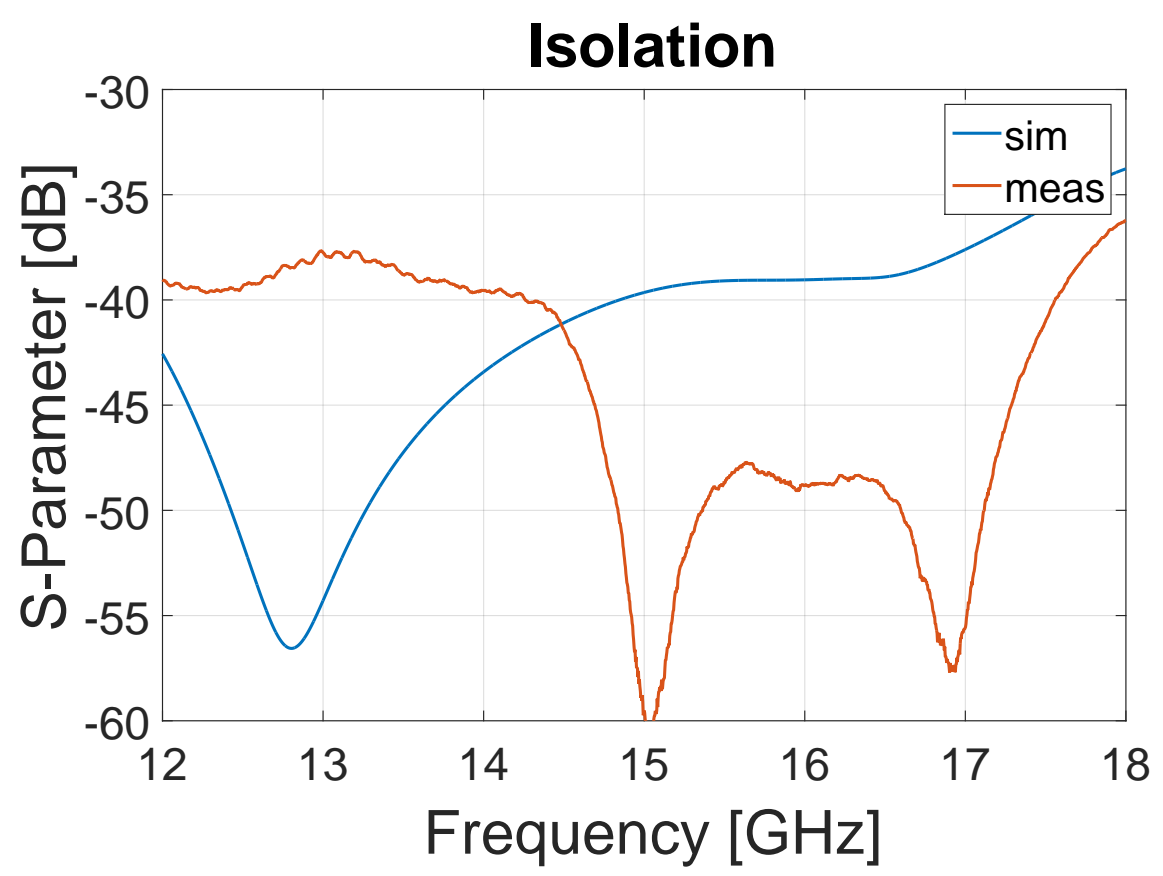

Figure 6.15: Comparison between measured and simulated Isolation (| $\left.S_{41} \mid\right)$. 


\subsection{Cross-guide Moreno directional coupler in empty substrate integrated}

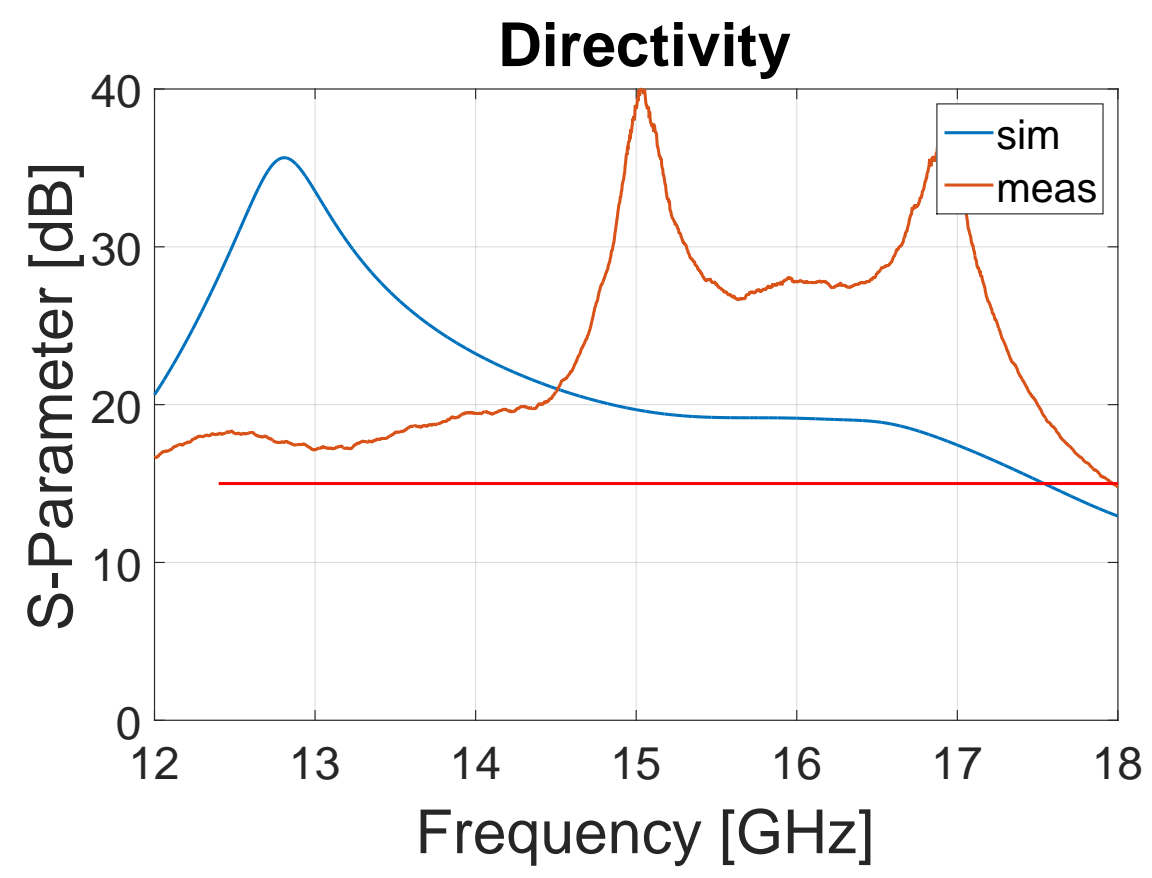

Figure 6.16: Calculated Directivity $\left(D=\left|S_{31}\right|-\left|S_{41}\right|\right)$.

Table 6.4: Comparison of broadband cross-guide couplers in planar form.

\begin{tabular}{cccc}
\hline Ref & {$[12]$} & {$[13]$} & This work \\
\hline Freq. & $28-38 \mathrm{GHz}$ & $26-40 \mathrm{GHz}$ & $12.4-18 \mathrm{GHz}$ \\
Coupling & $15 \mathrm{~dB}$ & $20 \mathrm{~dB}$ & $20 \mathrm{~dB}$ \\
Directivity & $15 \mathrm{~dB}$ & $10 \mathrm{~dB}$ & $15 \mathrm{~dB}$ \\
Data & Simulated & Measured & Measured including transitions \\
Technology & SIW & AFSIW & ESIW \\
\hline
\end{tabular}

a very interesting device at simulation level, unfortunately, fabrication and measurements were not performed. Transitions to a well-know transmission line, as coplanar or microstrip are needed in order to integrate the device with standard PCB circuits. These transitions, which worsen the directivity and teh insertion loss, are only included in the measurements of this work.

\subsubsection{Final Conslusions}

This chapter introduces a ESIW Slotted Antenna and a ESIW Cross-guide Coupler.

On one hand, the high efficient antenna measurement results fit the simulated data very well. The radiation efficiency improves the present state of the art (SIW slotted antenna) by removing the dielectric losses inside the substrate integrated waveguide. The antenna is 
suitable for the targeted application and ready to be integrated into a MIMO radar system.

On the other hand, a planar cross-guide directional coupler has been proposed. It is the first time that a cross-guide coupler is implemented in ESIW technology. High directivity and low return loss have been measured. The ESIW device implemented in this work has been proven to outperform other similar couplers in other empty substrate integrated waveguide technology in terms of directivity. ESIW is a promising technology in those applications where a traditional waveguide is utilized and smaller size would be beneficial as, for instance a complete MIMO Radar. A performance comparable to conventional waveguide (even more bandwidth of operation if transitions from waveguide to coaxial connector are mounted) is achieved with ESIW in a planar substrate, allowing a straightforward and easy integration with IC and substantially reducing its size. 


\section{Bibliography}

[1] A. Belenguer, H. Esteban, and V. Boria, "Novel empty substrate integrated waveguide for high-performance microwave integrated circuits.", in Microwave Theory and Techniques, vol. 62, no. 4, 832 - 839.

[2] M. Fernandez, J. Ballesteros and A. Belenguer, "Design of a hybrid directional coupler in empty substrate integrated waveguide (ESIW).", in Microwave and Wireless Components Letters, IEEE, vol. 25, no. 12, 796-798.

[3] J. Mateo, A.M. Torres, A. Belenguer and A.L. Borja, "Highly Efficient and wellMatched empty substrate integrated waveguide H-Plane horn antenna.", in IEEE Antennas and Wireless Propagation Letters, vol. 15, no. , pp. 1510-1513, 2016

[4] A.A. Khan, M.K. Mandal and R. Shaw, "A compact and wideband SMA connector to empty substrate integrated waveguide (ESIW) transition.", in 2015 IEEE MTT-S International Microwave and RF Conference (IMaRC), Hyderabad, 2015, pp. 246-248.

[5] J.V. Morro, A. Rodriguez, A. Belengue, H. Esteban and V. Boria, "Multilevel transition in empty substrate integrated waveguide. ", in Electronics Letters2015 IEEE MTT-S International Microwave and RF Conference (IMaRC), vol. 52, no. 18, pp. 1543-1544, 922016.

[6] E. Miralles, A. Belenguer, J. Mateo, A. Torres, H. Esteban, A.L. Borja and V. Boria, "Slotted ESIW Antenna with high efficiency for a MIMO Radar Sensor.", in Radio science, Accepted.

[7] E. Miralles, A. Belenguer, H. Esteban and V. Boria, "Cross-guide Moreno directional coupler in empty substrate integrated waveguide.", in Radio science,Vol. 52, Issue 5 May 2017 597603. DOI: 10.1002/2017RS006244.

[8] E. Miralles, T. Multerer, A. Ganis, B. Schoenlinner, U. Prechtel, A. Meusling, J. Mietzner, C. Weckerle, H. Esteban, M. Vossiek, M. Loghi, and V. Ziegler, "Multiductional and Compact 3D FMCW MIMO Radar System with Rectangular Array for Medium Range Applications" in IEEE Aerospace and Electronic Systems Magazine. Accepted.

[9] A. Belenguer, H. Esteban and V. Boria, "Novel empty substrate integrated waveguide for high-performance microwave integrated circuits." in Microwave Theory and Techniques, vol. 62, no. 4, 832 - 839. 
[10] J. Ball and T. Sulda, "Small aperture crossed waveguide broad-wall directional couplers." in Microwaves, Antennas and Propagation, IEE Proceedings, vol. 147, no. 4, 249-254.

[11] E. Miralles, V. Ziegler, U. Wochner, M. Zedler and F. Ellinger, "Frequency adjustable Ka band cross-guide coupler for space applications." in German Microwave Conference (GeMiC), 170-173.

[12] Y. Zhang, Q. Wang and J. Ding, "A cross-guide waveguide directional coupler with high directivity and broad bandwidth." in Microwave and Wireless Components Letters, IEEE, vol. 23, no. 11, 581-583.

[13] F. Parment, A. Ghiotto, T. Vuong, J. Duchamp and K. Wu, "Broadband directional Moreno coupler for high-performance air-filled SIW-based Substrate Integrated Systems." in Microwave Symposium (IMS), 2016 IEEE MTT-S International , pp. 1-3. 


\section{Chapter 7}

\section{Summary and Future Lines of Research}

\subsection{Summary}

In this industrial dissertation, a variety of new and creative ideas has been adressed.

In Chapter 2, a brief introduction to an existing MIMO radar theory as well as to the neccessary basic concepts of FMCW, TDM and angle estimation has been given.

After that, in Chapter 3 a complete and fully functional MIMO radar has been presented. The sandwich approach enabled to stack PCBs to make up a modular bi-dimensional array of very wideband TSA antennas. My contributions to this radar have been presented in detail as well as the final imaging results, in which the increment of the resolution due to the MIMO technique has succesfully been tested.

A next iteration of the portotype has been introduced in Chapter 4. This radar replaces the multiple stacked PCBs by a single one, which is populated with air-cavity-based RSPAs. This PCB has been designed with two independent switch chains, in such a manner that the two different waveforms can be sent simultaneously. Therefore, a real time radar, as for instance OFMD can be tested with this hardware. The characteristic inoccupied space at the center of a bidimentional MIMO array is filled with a second funtionality. Firstly, spiral antennas for communication have been designed, integrated and tested. Secondly, the spiral antennas have been cut off. In the remaining hole, a camara, which improves the detection, clasification and tracking of targets, has been placed.

Chapter 5 presents new, very broadband (4-40 GHz) and integrable-with-PCBs RF components as a Wilkinson divider and a 3D printed TEM horn antenna. These components have succesfuly been designed, fabricated and tested. The targeted wide frequency range allows the usage of these components for multiple functions. 
In Chapter 6 new low losses key components as the Moreno cross-guide coupler and slotted waveguide antenna are presented in a new very promising technology. ESIW reduces the loss of the integrated waveguides (SIW) by removing the inner dielectric, following a very interesting fabrication process. These two components have been, designed, fabricated and measured.

On one hand, the produced prototypes are the first step towards further Airbus refinement before introducing a product to the market. On the other hand, some disruptive technologies and components have been explored in order to keep track of the following advances in terms of innovation.

\subsection{Resumen}

En esta disertación industrial, se han abordado una variedad de nuevas ideas.

En el capítulo 2, se expone una breve introducción a la teoría básica de los radares tipo MIMO, así como también conceptos esenciales como FMCW, TDM o la estimación de ángulos.

En el siguiente capítulo, un radar de tipo MIMO es presentado. En el varias tarjetas de circuitos impresos son apiladas, creando de esta forma, una agrupación de antenas de gran ancho de banda bidimensional. Mis trabajo es presentado en este capítulo, así también como las imágenes radar finales. Al final, el incremento de resolución debido a la técnica MIMO se demuestra exitosamente. Una iteracin de el prototipo es presentado en el captulo 4. Este radar sustituye las múltiples tarjetas impresas por una sola, la cual contiene RSPA basadas en cavidades de aire. Este circuito se ha diseado con dos cadenas de conmutadores independientes, de tal forma que se dos formas de onda podrían ser enviadas de forma simultánea. Por ello, un radar en tiempo real, como por ejemplo un radar OFMD puede ser probado con este hardware. La característica zona vacía de los radares MIMO ha sido ocupada con una segunda funcionalidad. Primero, con antenas espirales para las comunicaciones y después a cámara, la cual mejora la detección, clasificación y el rastreo de objetivos.

El capítulo 5 introduce novedosos componentes de RF, de muy ancho de banda e integrables con tarjetas de circuito impreso como un Divisor Wilkinson y una antena impresa en 3D. Estos componentes han sido diseado, fabricado y medido de forma satisfactoria. El gran ancho de banda objetivo permite a estos componentes operar para múltiples funciones.

El capítulo 6 introduce nuevos componentes clave de bajas pérdidas como el acoplador Moreno y la antena de guía de ondas ranurada en una nueva y prometedora tecnología. ESIW reduce las pérdidas de las guías de ondas integradas eliminando el dieléctrico in- 
terno. Estos componentes han sido diseados, fabricados y medidos.

Los prototipos producidos son un primer paso antes de un refinamiento final antes de introducir el producto al mercado. Además, se han explorado tecnología ms disruptivas para futuros proyectos.

\subsection{Resum}

En aquesta dissertació industrial, s'han abordat una varietat de noves idees.

En el capítol 2, s'exposa una breu introducció a la teoria bàsica dels radars tipus MIMO i conceptes essencials com FMCW, TDM o l'estimació d'angles.

En el següent capítol, un radar de tipus MIMO és presentat. Diverses targetes de circuits impresos són apilades, creant d'aquesta manera, una agrupació d'antenes bidimensional de gran ample de banda. El meu treball és presentat en aquest capítol, així també com les imatges radar finals. Al final, l'increment de resolució a causa de la tècnica MIMO es demostra amb èxit. La següent iteració del prototip és presentada al capítol 4. Aquest radar substitueix les múltiples targetes impreses per una sola, la qual conté RSPA basades en cavitats d'aire. Aquest circuit s'ha dissenyat amb dues cadenes de commutadors independents, de tal manera que dues formes d'ona podrien ser enviades de forma simultània. Per aixó, un radar en temps real, com per exemple un radar OFMD pot ser provat amb aquest radar. La característica zona buida dels radars MIMO ha estat ocupada amb una segona funcionalitat. Primer, amb antenes espirals per a les comunicacions i després per a cmera, la qual millora la detecció, classificació i el rastreig d'objectius.

El capítol 5 introdueix nous components de RF, de molt ample de banda i integrables amb targetes de circuit imprès com un Divisor Wilkinson i una antena impresa en 3D. Aquests components han estat dissenyats, fabricats i mesurats de forma satisfactòria. El gran ample de banda objectiu permet a aquests components operar per a múltiples funcions.

El capítol 6 introdueix nous components clau de baixes pèrdues com el acoblador Moreno i l'antena de guia d'ones ranurada en una nova i prometedora tecnologia. ESIW redueix les pèrdues de les guies d'ones integrades eliminant el dielèctric intern. Aquests components han estat dissenyats, fabricats i mesurats.

Els prototips produts són un primer pas abans d'un refinament final abans d'introduir el producte al mercat. A més, s'han explorat tecnologia més disruptives per a futurs projectes. 


\subsection{Future Lines of Research}

There are various topics worthy of future research.

The two presented radars are based in a TDM scheme. Therefore, the antennas achieve orthogonality by capturing the information in different time frames. In a very fast environment, it produces inaccuracies, since the information captured in the different antennas correspons to slightly different environments. A way to solve this issue is to achieve orthogonality by means of sending a different waveform through different antennas simultaneously. The second radar has two independent switch chains in such a way that this concept can be tested in the future.

In Chapter 5, a very broadband Wilkinson divisor and a very broadband 3D-printed TEM horn antenna have been presented. Nowadays, there are also commercial switches and amplifiers covering this wide frequency range. A fully operational radar prototype, working from 4 to $40 \mathrm{GHz}$ would be very interesting because of three aspects. The same hardware can be used in the whole world, bypassing frequency regulations. Very broadband modulations, which improve the range resolution accuracy, could be used. Hopping frequency technoques could be used in this radar, in such a way that the probability of dectecting and intercepting the radar would drecrease.

In Chapter 6, two ESIW passive components have been designed, fabricated and tested. The two fabricated components enable a significant reduction of the power comsumption of the complete radar, since they produce lower losses than traditional components based on microstrip. A fully operational MIMO radar, which includes ESIW passive components connected with commertial IC is very interesting for a future research.

The fabricated antenna in Chapter 5 is made up of two 3d-printed metallic parts, a potential improvement would be achieved by printing a single piece of plastic and to partially metallize its sides, in such a way the antenna dimensions would be reduced. A low $\tan (\delta)$ printable material would also be needed. A patent on this idea has been published [1].

\subsection{List of Contributions}

- A. Ganis, E. Miralles, B. Schoenlinner, U. Prechtel, A. Meusling, C. Heller, T. Spreng, J. Mietzner, C. Krimmer, B. Haeberle, M. Maier, S. Lutz, C. Weckerle, H. P. Feldle, M. Loghi, A. Belenguer, H. Esteban and V. Ziegler "A Portable 3D Imaging FMCW MIMO Radar Demonstrator with a Modular 24x24 Antenna Array" IEEE Trans. Microw. Theory Techn.

- E. Miralles, T. Multerer, A. Ganis, B. Schoenlinner, U. Prechtel, A. Meusling, J. Mietzner, C. Weckerle, H. Esteban, M. Vossiek, M. Loghi, and V. Ziegler, "Multi- 
ductional and Compact 3D FMCW MIMO Radar System with Rectangular Array for Medium Range Applications" IEEE Aerospace and Electronic Systems Magazine. Accepted.

- E. Miralles, D. Rieth, B. Schoenlinner, U. Prechtel, A. Meusling, A. Belenguer, H. Esteban and V. Ziegler, "Low Cost Multipurpose RF System Approach: Integration of a MIMO Radar and a Communications Frontend on a Single PCB", in IEEE Microwave Conference (EuMC), 2016 European, London, UK, Oct 2016.

- E. Miralles, B. Schoenlinner, F.Ellinger and V. Ziegler "Fast Design Method and Validation of Very Wideband Tapered Wilkinson Divider" in Microwave Conference (EuMC), 2015 European, Paris, 2015, pp. 119-122.

- E. Miralles and V. Ziegler, "Circuit board for hf applications including an integrated broadband antenna", Publication number: US20170179607A1 (USA), DE102015225496A1 (GER) and EP3182504A1 (EU).

- E. Miralles, V. Ziegler, U. Wochner, M. Zedler and F. Ellinger, "Frequency adjustable Ka band cross-guide coupler for space applications." in German Microwave Conference (GeMiC), 170-173.

- A. Ganis, E. Miralles, C. Heller, U. Prechtel, A. Meusling, H. H. Feldle, M. Loghi, F. Ellinger and V. Ziegler, "A system concept for a 3D real-time OFDM MIMO radar for flying platforms." in German Microwave Conference (GeMiC), 201-204.

- E. Miralles, A. Belenguer, J. Mateo, A. Torres, H. Esteban, A.L. Borja and V. Boria, "Slotted ESIW Antenna with high efficiency for a MIMO Radar Sensor.", in Radio science, Submitted.

- J. Mietzner, S. Lutz, C. Weckerle, B. Hofstaetter, E. Miralles, A. Ganis, T. Multerer, J. Puchinger, U. Prechtel, V. Ziegler and A. Meusling, "Compact 3D MIMO radar Antenna array design and experimental results.", in 2017 European Radar Conference (EURAD), Nuremberg, 2017, pp. 130-133. DOI: 10.23919/EURAD.2017.8249164.

- T. Multerer, A. Ganis, U. Prechtel, E. Miralles, A. Meusling, J. Mietzner, M. Vossiek, M. Loghi and V. Ziegler "Low-cost jamming system against small drones using a 3D MIMO radar based tracking.", in 2017 European Radar Conference (EURAD), Nuremberg, 2017, pp. 299-302. DOI: 10.23919/EURAD.2017.8249206.

- M. Elsasser, E. Miralles, V. Ziegler and L. Vietzorreck "Broadband Microstrip to Waveguide Transition", in 23nd URSI GASS, Montreal, 19-26 August 2017. 


\subsection{List of abbreviations}

$\begin{array}{ll}\text { ADC } & \text { Analog-to-Digital Converter } \\ \text { AGI } & \text { Airbus Group Innovations } \\ \text { ATC } & \text { Air Trafic Control } \\ \text { AWR } & \text { Advancing the Wireless Revolution } \\ \text { CPLD } & \text { Complex Programmable Logic Device } \\ \text { CST } & \text { Computer Simulation Technology } \\ \text { DBF } & \text { Digital Beam Forming } \\ \text { DC } & \text { Direct Current } \\ \text { DDS } & \text { Direct Digital Synthesizer } \\ \text { DUT } & \text { Device Under Test } \\ \text { ENIG } & \text { Electroless Nickel Immesion Gold } \\ \text { ESIW } & \text { Empty Substrate Integrated Waveguide } \\ \text { FFT } & \text { Fast Fourier Transformation } \\ \text { FMCW } & \text { Frequency Modulated Continuous Wave } \\ \text { FOV } & \text { Field Of View } \\ \text { FPGA } & \text { Field Programmable Gate Array } \\ \text { HMI } & \text { Human-Machine Interface } \\ \text { IC } & \text { Integrated Circuit } \\ \text { ITU } & \text { International Telecommunication Union } \\ \text { LNA } & \text { Low Noise Amplifier } \\ \text { LO } & \text { Local oscillator } \\ \text { MIMO } & \text { Multiple Input Multiple Output } \\ \text { MSL } & \text { MicroStrip Line } \\ \text { NASA } & \text { National Aeronautics and Space Administration } \\ \text { NF } & \text { Noise Figure } \\ \text { OCP1 } & \text { 1 dB Output Compression Point } \\ \text { PCB } & \text { Printed Circuit Board } \\ \text { PLL } & \text { Phase-Locked Loop } \\ \text { RADAR } & \text { RAdio Detection And Ranging } \\ \text { RCS } & \text { Radar Cross Section } \\ \text { RF } & \text { Radio Frequency } \\ \text { RISC } & \text { Reduced Instruction Set Computer } \\ \text { RSPA } & \text { Resonant Slot Patch Antennas } \\ & \end{array}$




$\begin{array}{ll}\text { RX } & \text { Receive } \\ \text { SAR } & \text { Synthetic-Aperture Radar } \\ \text { SLL } & \text { Side Lobe Level } \\ \text { SMA } & \text { Sub-Miniature version A } \\ \text { SoC } & \text { System on Chip } \\ \text { SOLT } & \text { Short-Open-Load-Thru } \\ \text { SWaP } & \text { Size, Weight and Power } \\ \text { TDM } & \text { Time Domain Multiplexing } \\ \text { TOI } & \text { Third Order Intercept } \\ \text { TRL } & \text { Technology Readness Level } \\ \text { TSA } & \text { Tapered Slot Antennas } \\ \text { TX } & \text { Transmit } \\ \text { UAV } & \text { Unmanned Aerial Vehicles } \\ \text { UK } & \text { United Kingdom } \\ \text { UPV } & \text { Universitat Politecnica de Valencia } \\ \text { USA } & \text { United States of America } \\ \text { USSR } & \text { Union of Soviet Socialist Republics } \\ \text { UWB } & \text { Ultra-WideBand }\end{array}$




\section{Bibliography}

[1] E. Miralles and V. Ziegler, "Circuit board for hf applications including an integrated broadband antenna", Publication number: US20170179607A1 (USA), DE102015225496A1 (GER) and EP3182504A1 (EU). 


\section{Gracias.}

La ironía de usar letras para expresar lo inefable, es la tarea que ahora me atañe. Tantas son las personas que navegan conmigo en este río que desemboca al mar... En primer lugar, agradezco a mi familia por darme vida, ganas de vivir y los recursos para permitir que cumpla todo aquello que me proponga. No hay obstáculos, ni fronteras, ni distancias, ni pasado, ni futuro, que cambie algo tan estructural como la familia. Al final, no es más que el principio de la esencia de uno mismo. En segundo lugar, mis docentes, especialmente aquellos que han trabajado con el corazón en la mano, intentando hacer un mundo mejor. Desafortunadamente, el caudal del fluir de mi tiempo empuja mi barca y no me permite mirar la orilla tanto como me gustaría. En tercer lugar, mis amigos, tengo suerte de tener pocos, pero buenos. Siempre me habéis acompañado y escuchado en los malos momentos, mientras hemos disfrutado y nos hemos reído en los buenos. Sin amigos con los que compartir, la vida no tiene sentido.

Muchos claroscuros han hecho presencia durante el transcurso de este viaje, pero los valles son necesarios para que puedan existir los picos, en este equilibrado desequilibro que evita el encefalograma plano. Gracias, por todo. Gracias a todos. Y gracias a ti que te molestas en leer estas lineas. 